\title{
A cell-based NRG1-ERBB4 assay designed for high- throughput compound screening to identify small molecule modulators with relevance for schizophrenia
}

\section{Dissertation}

for the award of the degree

'Doctor rerum naturalium'

of the Georg-August-Universität Göttingen

submitted by

Wilko Hinrichs

from Norderney, Germany

Göttingen, 30.09.2012 
Prof. Klaus-Armin Nave Ph.D. (Reviewer)

Department of Neurogenetics, Max Planck Institute of Experimental Medicine, Göttingen

\section{Prof. Dr. Martin Göpfert (Reviewer)}

Department of Cellular Neurobiology, Schwann-Schleiden Research Center, Göttingen

\section{Prof. Dr. André Fischer (Reviewer)}

Laboratory for Aging and Cognitive Diseases, European Neuroscience Institute, Göttingen

\section{PD. Dr. Moritz Rossner}

Department of Neurogenetics, Max Planck Institute of Experimental Medicine, Göttingen

Prof. Dr. Thomas A. Bayer

Department of Psychiatry, Division of Molecular Psychiatry, UKG Göttingen

\section{Dr. Judith Stegmüller}

Department of Cellular and Molecular Neurobiology, Max Planck Institute of Experimental Medicine, Göttingen

Declaration

Herewith I declare that I prepared the PhD thesis entitled:'A cell-based NRG1ERBB4 assay designed for high-throughput compound screening to identify small molecule modulators with relevance for schizophrenia' on my own and with no other sources and aids than quoted. 
"We are as forlorn as children lost in the woods. When you stand in front of me and look at me, what do you know of the griefs that are in me and what do I know of yours. And if I were to cast myself down before you and weep and tell you, what more would you know about me than you know about Hell when someone tells you it is hot and dreadful? For that reason alone we human beings ought to stand before one another as reverently, as reflectively, as lovingly, as we would before the entrance to Hell."

Franz Kafka 


\section{Acknowledgments}

First, I want to thank my supervisor PD. Dr. Moritz Rossner, for the great PhD project, his support, and all his brilliant ideas and good advice accumulated in this thesis.

Equally, I want to thank my co-supervisor Dr. Michael Wehr, for his friendship, for his patience, for the great discussions, the help with the projects, especially screening and biochemistry, and the proof reading of my thesis.

Dr. Sven Wichert for the help with the Hamilton robot, all bioinformatical questions, and help with all computer-related problems.

Dr. Magdalena Brzozka for discussions, help with the corrections and the deeper insight into mouse behavioural studies.

Dr. Elena Ciirdaeva for great support with cloning and supplies of DNA.

All members of the Rossner group and the Department of Neurogenetics, for friendship, help in all lab problems concerning techniques, instruments, software, and discussion of projects.

The members of the Lead Discovery Centre Dortmund, particularly Dr. Sascha Menninger for the great co-operation, and a deeper insight into HTP screening, lead discovery, and lead optimisation

The GGNB-CMPB program, specifically Prof Michael Hörner, for the fruit-full discussions and seminar series and the GGNB staff for the help with the administrative challenges.

I am grateful for my PhD committee, including Prof. Martin Göpfert, and Prof. André Fischer, and especially Prof. Klaus-Armin Nave for discussions, encouragements, and having me as PhD student in his lab.

Further thanks are to my parents, without there love, support and encouragement this thesis would not be possible.

Carolin, for her love and support in this part of my life.

All friends, fencers, and tabletop-wargamers for the life abroad the PhD. 


\section{Table of Content}

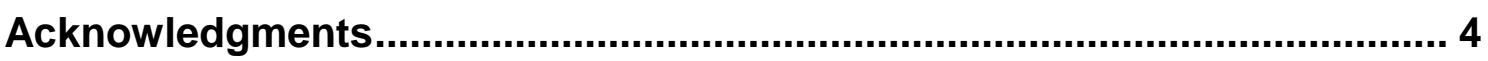

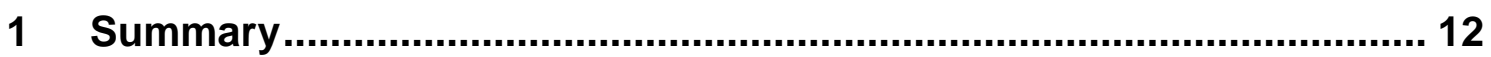

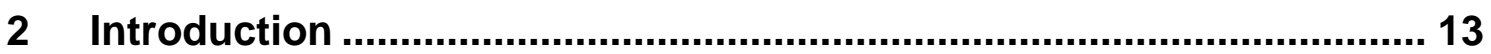

2.1 General characterisation of schizophrenia ……...................................... 13

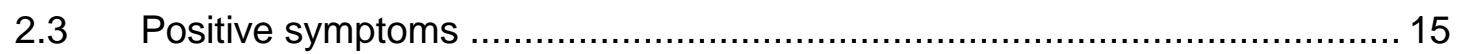

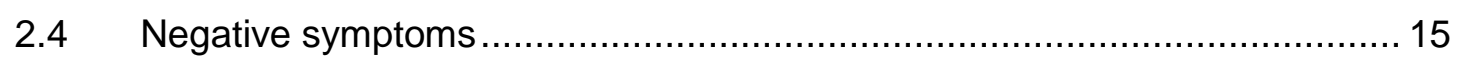

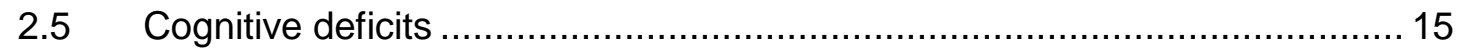

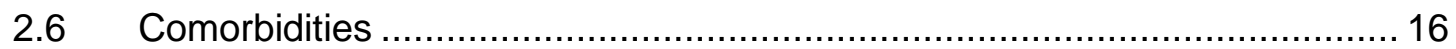

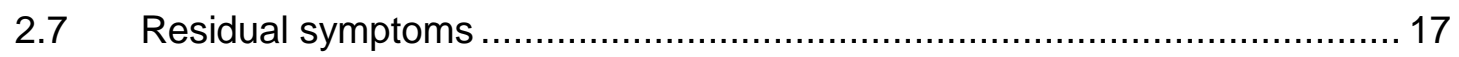

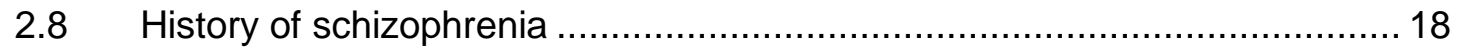

2.9 Morphologic findings in schizophrenia ………........................................ 19

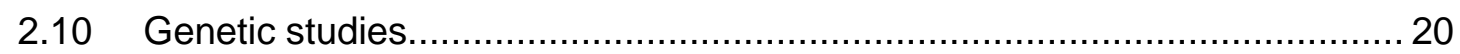

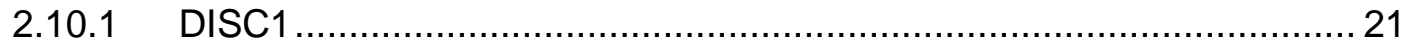

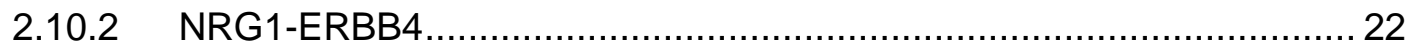

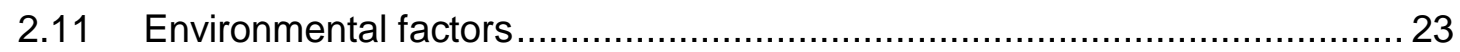

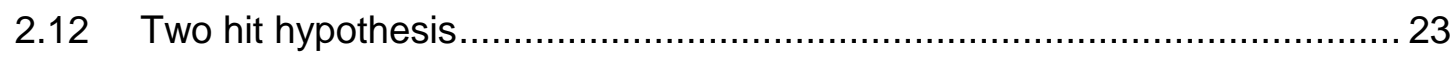

2.13 Findings in animal models of schizophrenia .............................................. 24

2.13.1 Dopamine hypothesis of schizophrenia......................................... 24

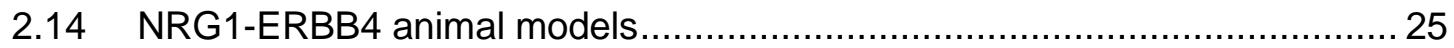

2.15 NRG1-ERBB4: from proteins to network analysis ....................................28

2.15.1 Why addressing the NRG1-ERBB4 signalling system? ....................... 28

2.15.2 Protein-protein-interactions and NRG1-ERBB4 signalling ................... 28

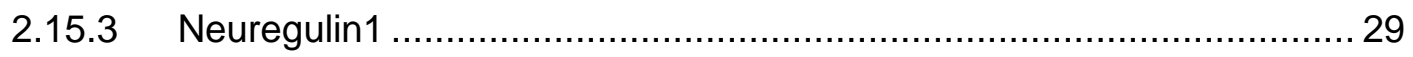

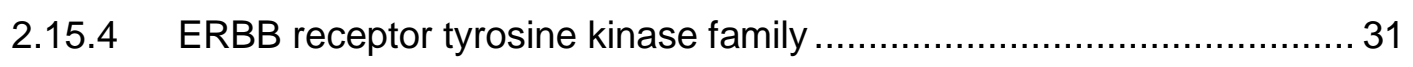

2.15.5 NRG1-ERBB4 signalling in the nervous system................................35

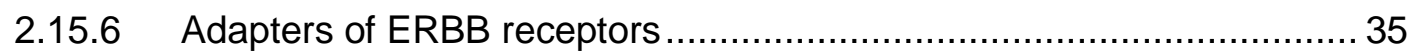

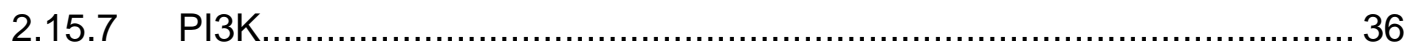

2.15 .8 STAT5A

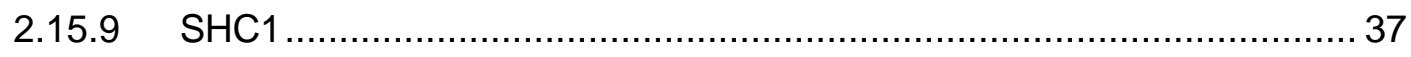

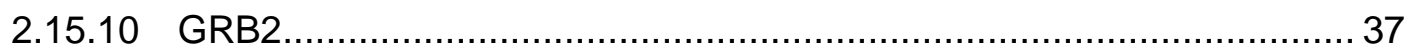

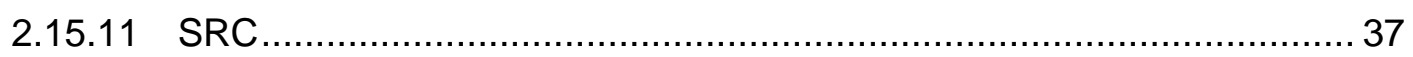

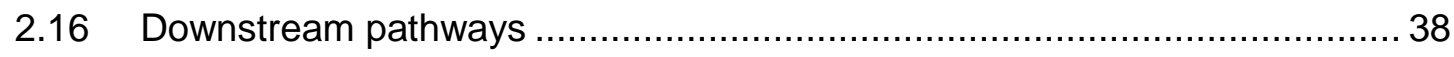




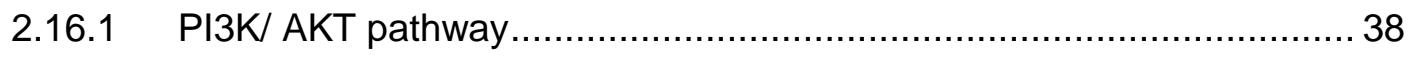

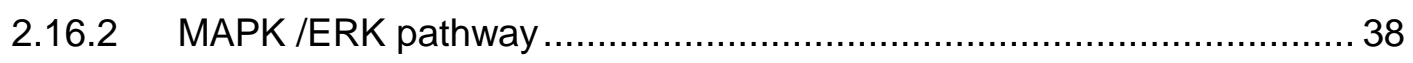

2.17 Localisation of NRG1-ERBB4 signalling in the neuronal architecture ..........40 40

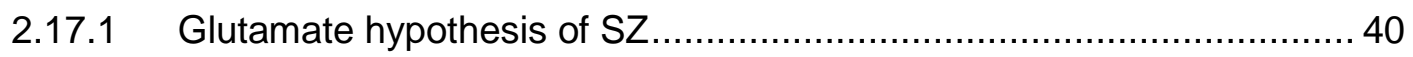

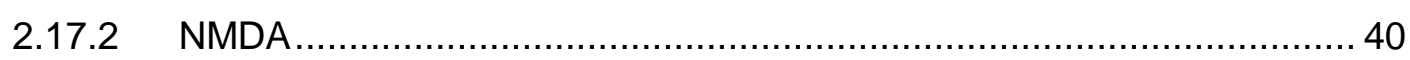

mGLu2/3 40

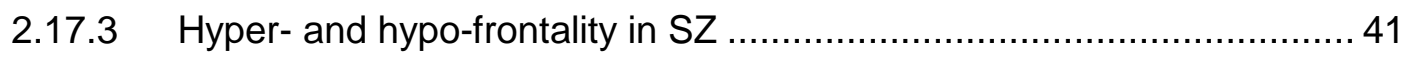

2.17.4 From neuronal circuits to single synapses ......................................... 41

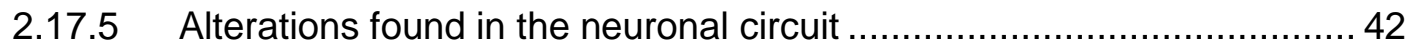

2.17.6 NRG1-ERBB4 in the development of PV+ interneurons...................... 43

2.18 Targeting NRG1-ERBB4 with protein-protein interaction assays ................. 45

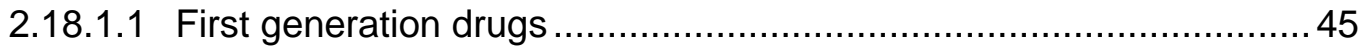

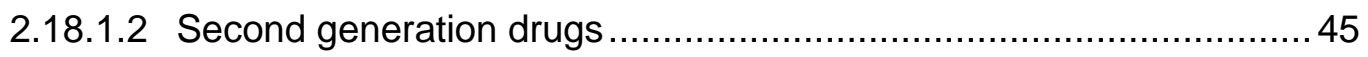

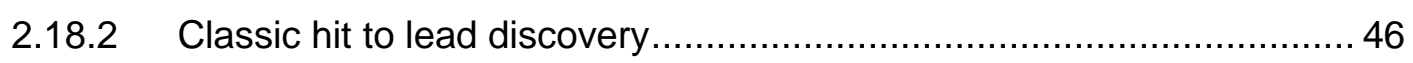

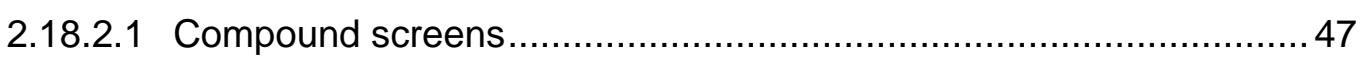

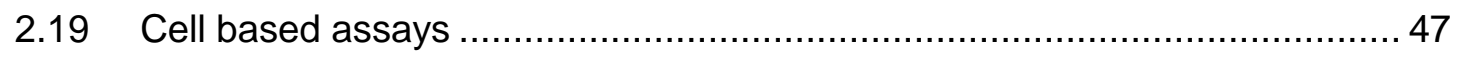

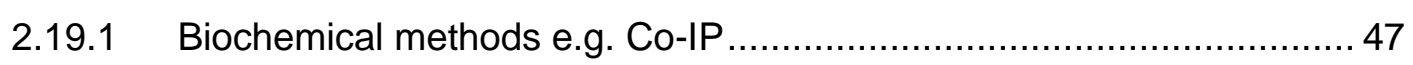

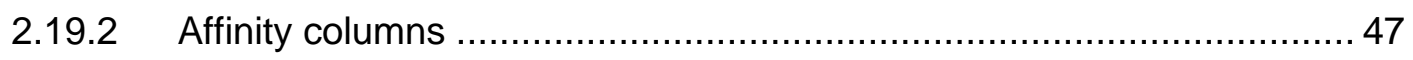

2.20 Protein fragment complementation assays ............................................... 48

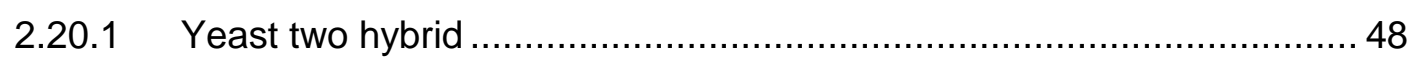

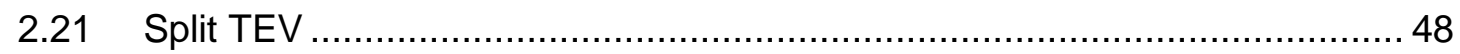

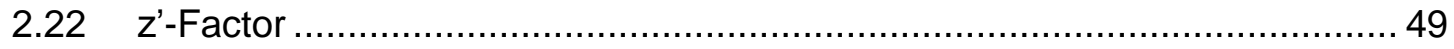

2.22.1 The interpretation of the $z$ 'factor: ...................................................... 49

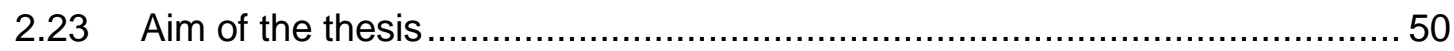

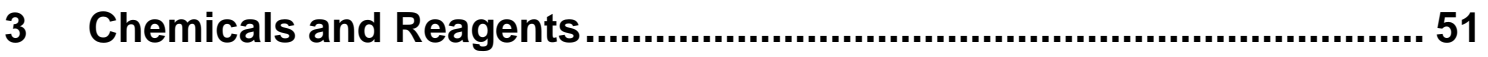

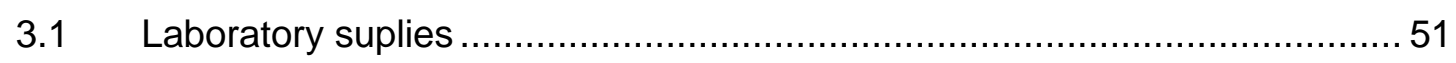

3.2 Laboratory equipment...................................................................... 51

Hardware51

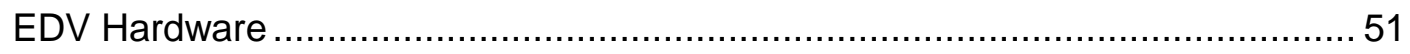

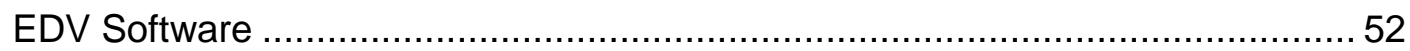

Kits $\quad 52$

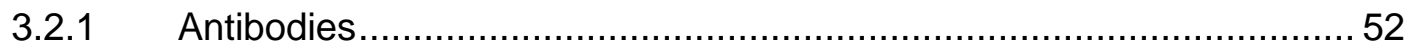

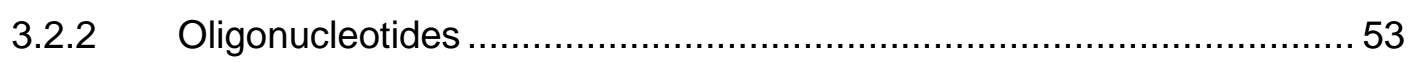

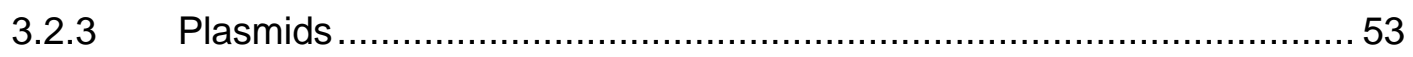

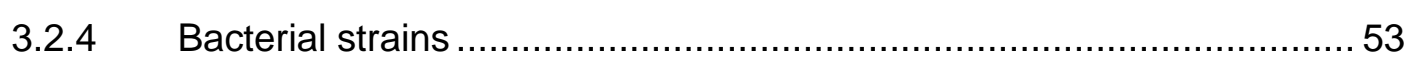

3.2.5 Mammalian cell lines .................................................................. 54 


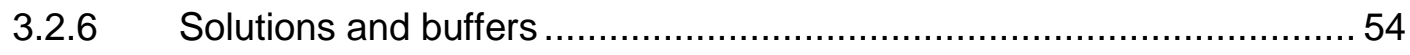

3.2.7 Buffers for molecular biology ….............................................. 54

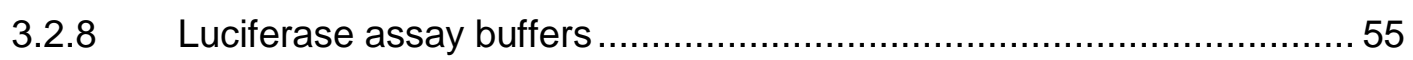

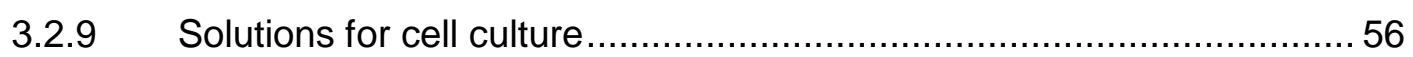

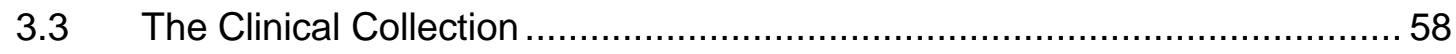

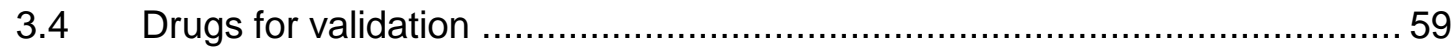

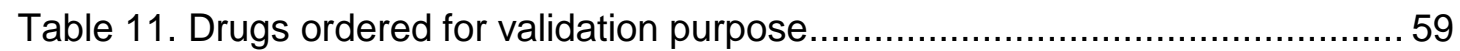

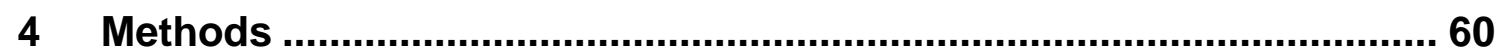

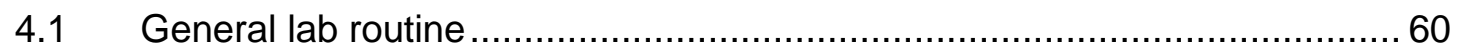

4.2 Transformation of chemically competent bacteria ...................................6 60

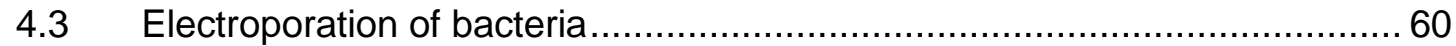

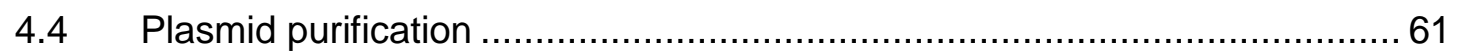

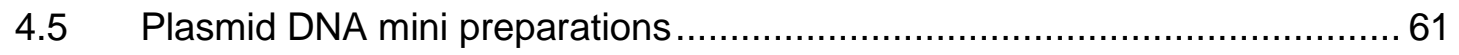

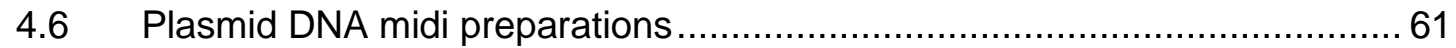

4.7 Photometric concentration determination of nuclear acids ......................... 61

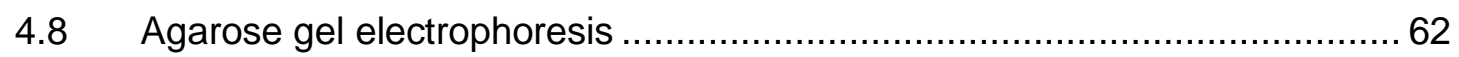

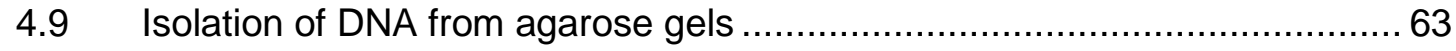

4.10 DNA digest with restriction endonucleases .............................................. 64

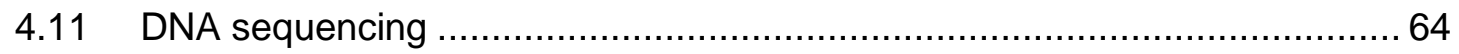

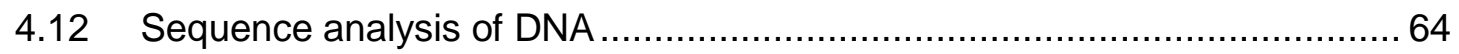

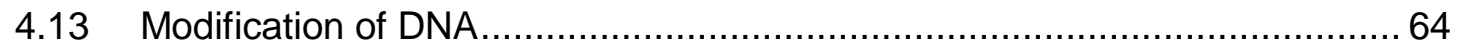

4.14 Dephosphorylation of 5'-DNA fragment overhangs, vectors only ................. 65

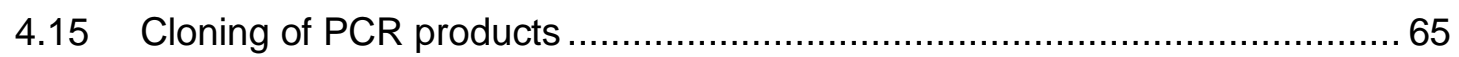

4.16 Amplification of DNA by polymerase chain reaction .................................. 66

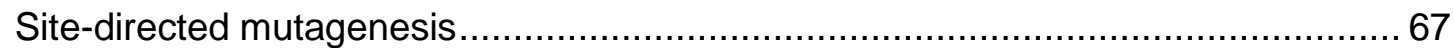

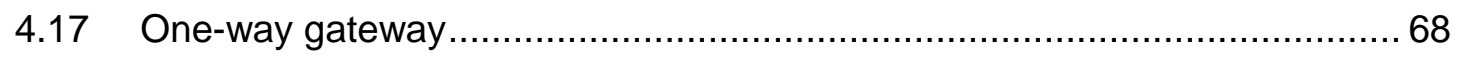

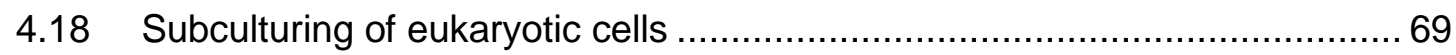

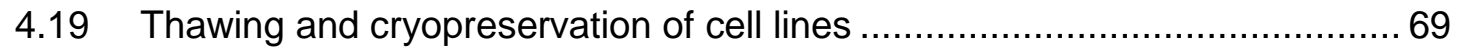

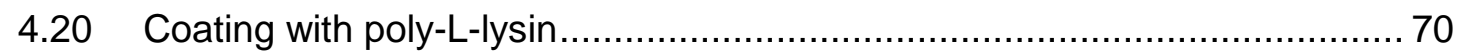

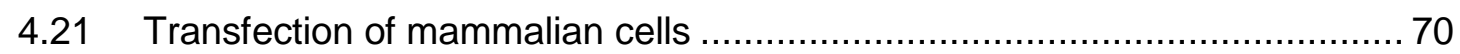

4.22 DNA transfer in eukaryotic cells with electroporation ............................... 71

4.23 Generation of stable cell lines............................................................ 71

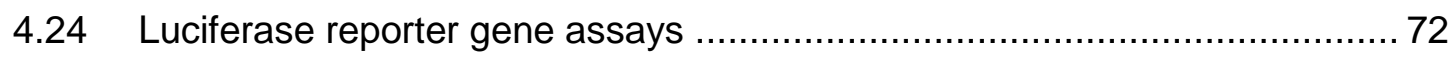


4.24.1 Single assay 16x6 measurement points....................................... 72

4.24.2 Normalisation and transfection control .......................................... 73

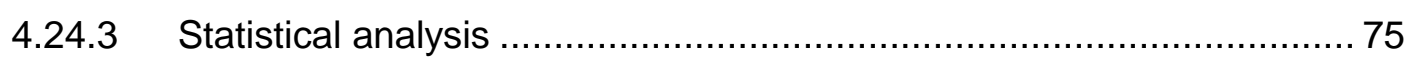

4.25 Screening of high throughput assays 96 well formats ............................... 75

4.25.1 Automated PLL coating......................................................... 75

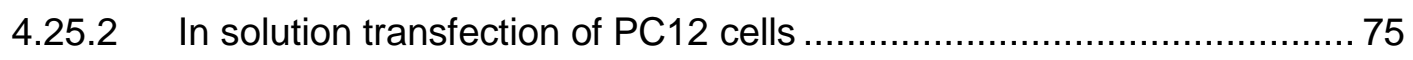

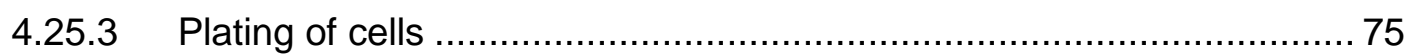

4.25.4 Handling of stable Nrg1-type-I expressing cells ............................... 76

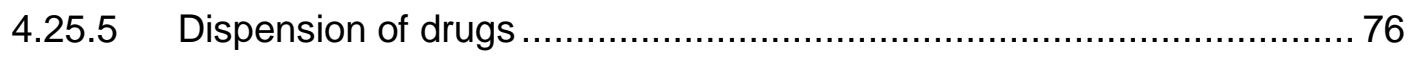

4.25.6 Automated dual luciferase assay ............................................... 77

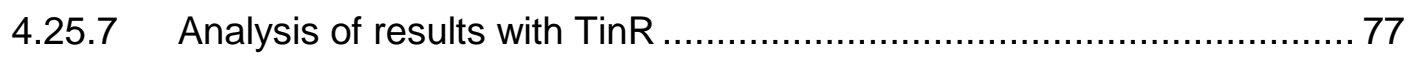

4.25.8 Preparation of dose response dilutions for 96 well format.................... 80

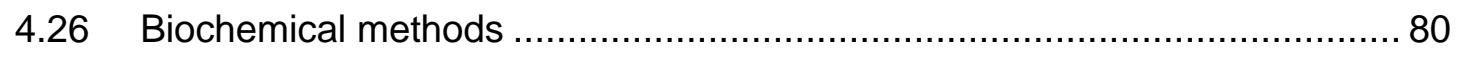

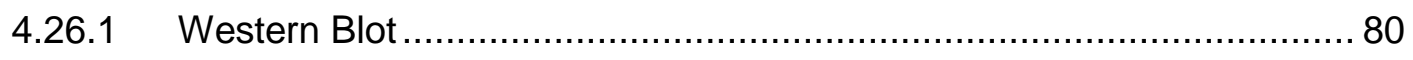

4.26.2 Sodium dodecyl sulphate polyacrylamid gel electrophoresis (SDSPAGE) 80

4.26.3 Transfer of proteins on membranes ................................................. 81

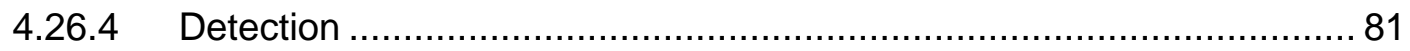

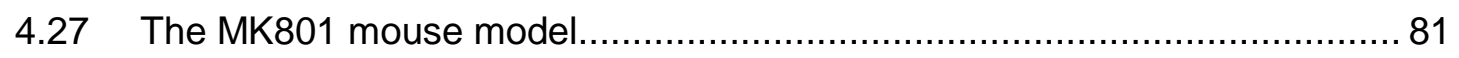

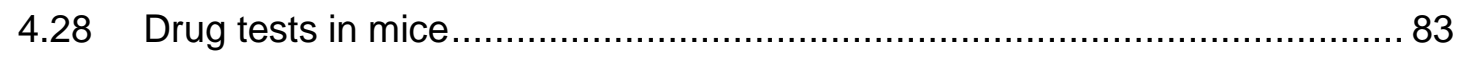

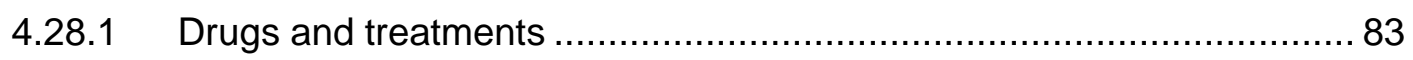

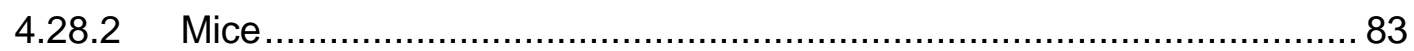

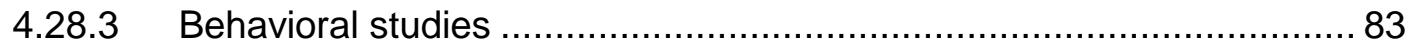

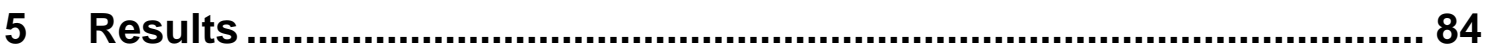

5.1 Design of a screening platform for Nrg1-ERBB4 signalling ........................ 84

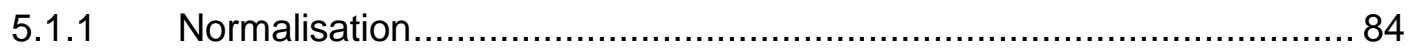

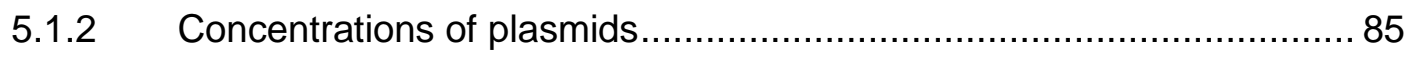

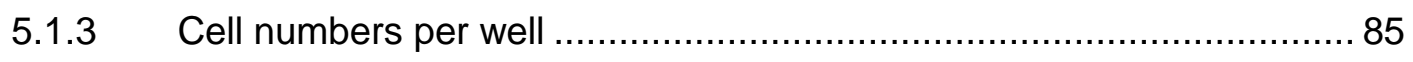

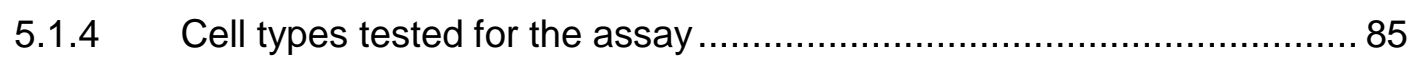

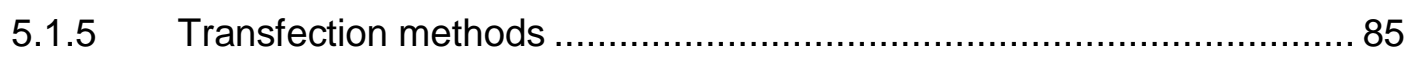

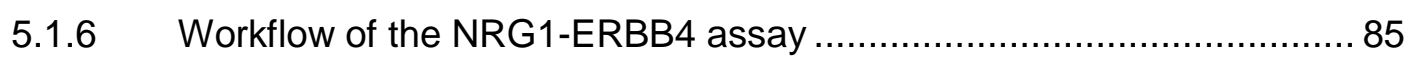

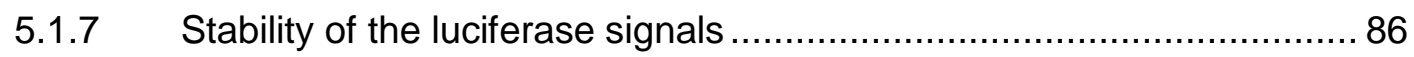

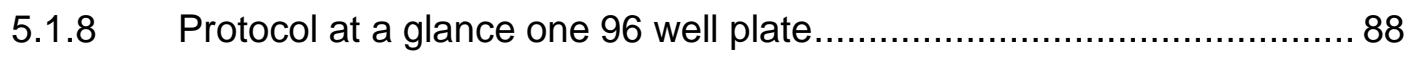

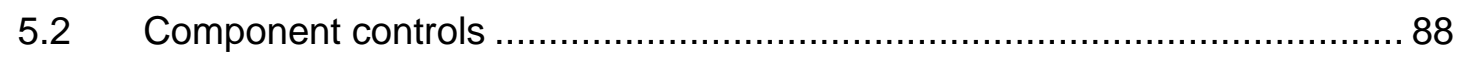

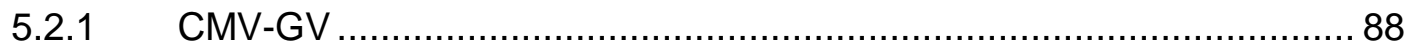

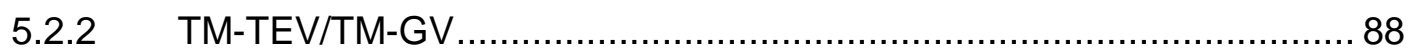




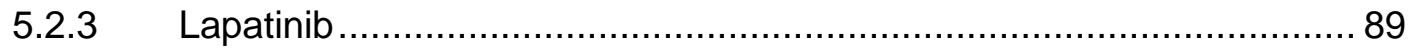

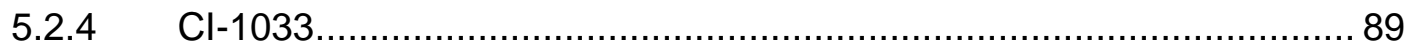

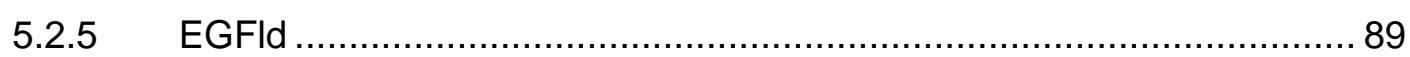

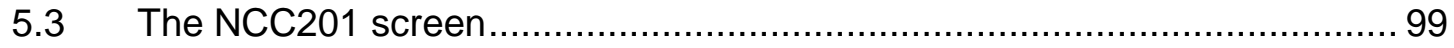

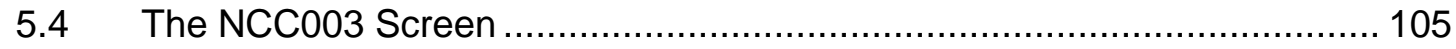

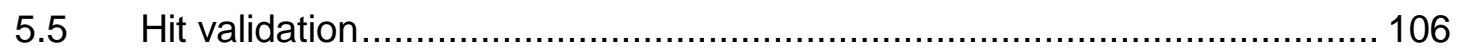

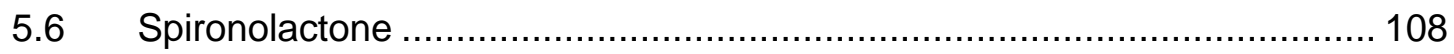

5.6.1 Vertical validation for Spironolactone ......................................... 110

5.6.2 Technical controls for Spironolactone validation .............................. 110

5.6.2.1 Renilla luciferase ...................................................................... 110

5.6.2.2 The Gal4-VP16 control assay ................................................. 110

5.6.2.3 TEV protease control assay ................................................... 110

5.6.2.4 1-cell assay with soluble Nrg1-EGF-like domain .........................111

5.6.3 Horizontal validation ........................................................... 112

5.6.3.1 ERBB4 receptor dimerisations ............................................. 112

5.6.3.2 ERBBx/ERBBy ................................................................. 112

5.6.3.3 ERBB2/ERBB4 dimerisation and adapters ................................. 112

5.6.3.4 ERBB2/ERBB3 dimerisation and adapters .................................112

5.6.3.5 ERBB4/PIK3R1 Aldosterone derivatives .....................................112

5.6.3.6 ERBB4/PIK3R1 Eplerenone and Canrenone ............................... 113

5.6.4 Orthogonal validation ............................................................. 113

5.6.4.1 ERBB1/ERBB1/EGF .......................................................... 113

5.6.4.2 GPCR Serotonin receptor 5A (HTR5A) activation ........................113

5.6.4.3 FRB/FKBP model interaction induced by Rapamycin...................113

5.6.5 Summary $\mathrm{IC}_{50}$ validation Spironolacone...................................... 114

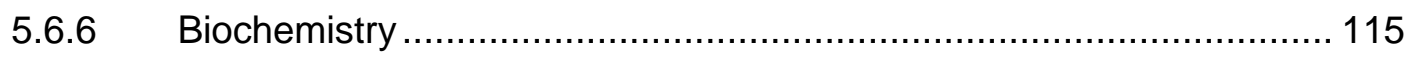

5.6.7 Validation in HEK293 cells ......................................................... 115

5.6.8 Validation in a MK801 mouse model of psychosis ........................... 116

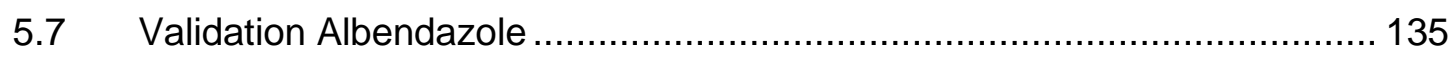

5.7.1 Technical controls for Albendazole ................................................ 135

5.7.1.1 Renilla Luciferase ................................................................. 135

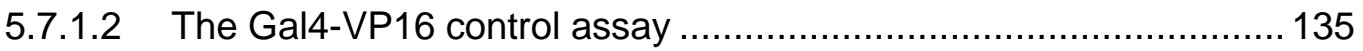

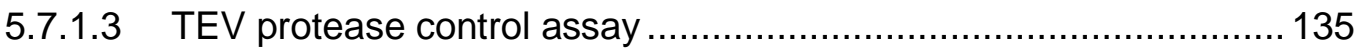

5.7.1.4 Co-culture assay increasing numbers of Nrg1-typel cells.............. 135

5.7.1.5 Single cell assay with soluble Nrg1-derived EGF-like domain ........136

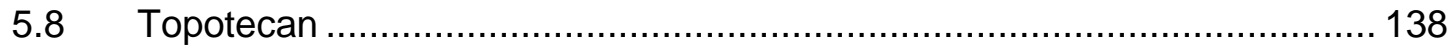

5.8.1 Vertical validation for Topotecan .......................................... 138 


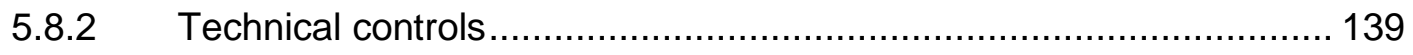

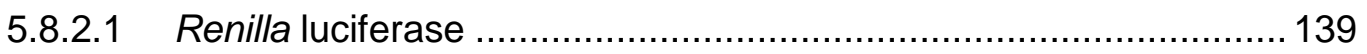

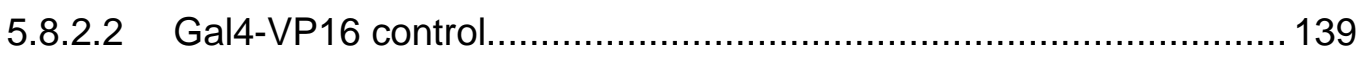

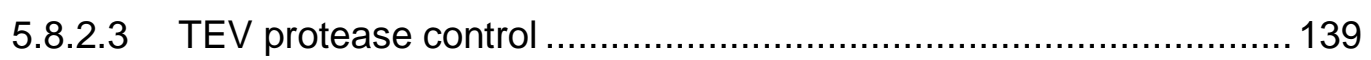

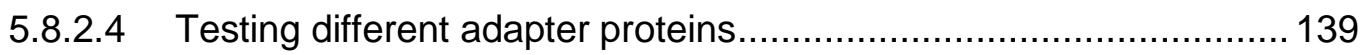

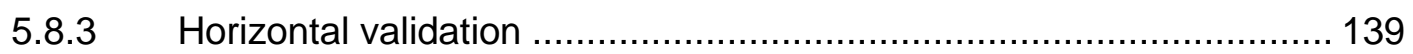

5.8.3.1 ERBB2/ERBB4 dimerisation and adapters................................... 140

5.8.3.2 ERBB2/ERBB3 dimerisation and adapters.................................. 140

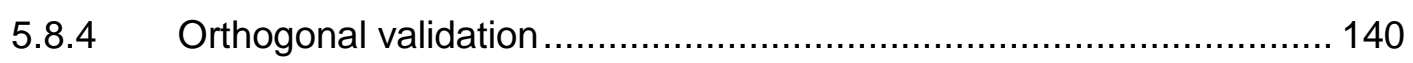

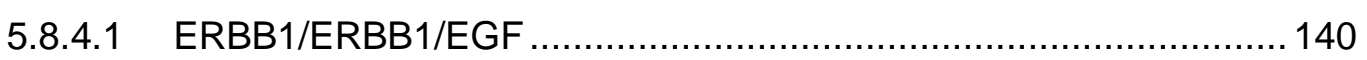

5.8.4.2 FRB/FKBP model interaction induced by Rapamycin.................... 140

5.8.4.3 ERBB4/PIK3R1 assay and effects caused by Irinotecan and SN38140

5.8.4.4 Summary validation Topotecan .................................................. 141

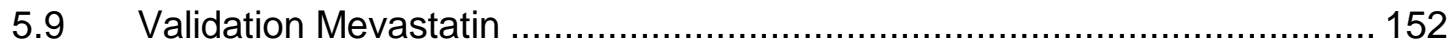

5.9.1 Technical controls for Mevastatin.................................................. 152

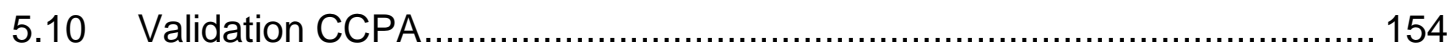

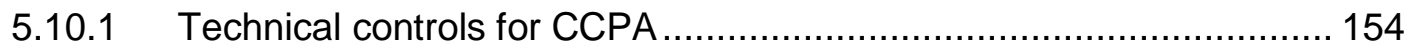

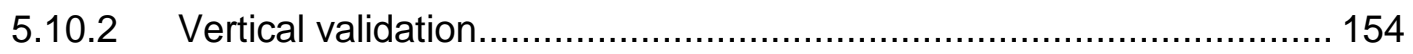

5.11 Validation Vincristine, effects on Renilla luciferase ................................ 156

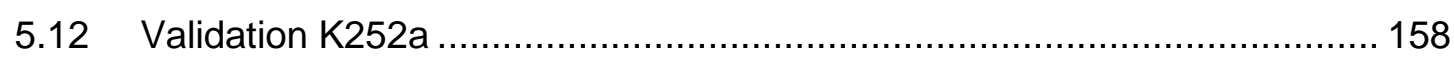

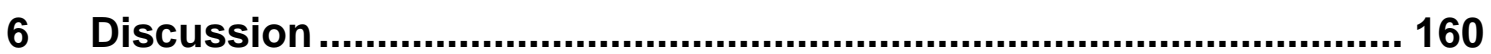

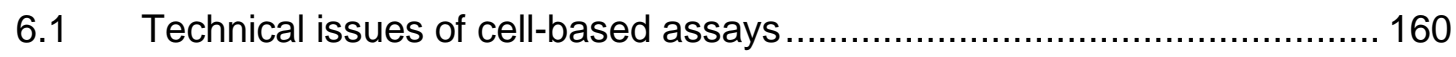

6.1.1 Translational assays and using GWAS data to model SZ ............... 160

6.1.2 Rational design of the assay workflow .......................................... 160

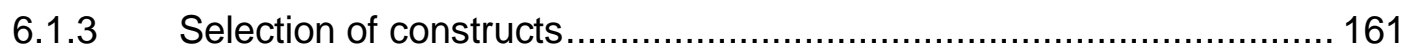

6.1.4 Artificial and tagged proteins in the cell-based assay ........................ 162

6.1.5 In vitro screens vs. co-culture screening systems .............................. 162

6.1.6 Limitations of co-culture systems vs. animal models ........................ 163

6.1.7 Screening of compound libraries: The hit-to-lead process in drug discovery 164

6.1.8 Screening of FDA approved drug libraries ..................................... 164

6.1.9 Transfer of the HTP screen to the LDC ............................................. 164

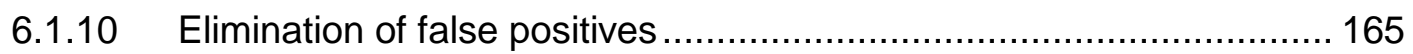

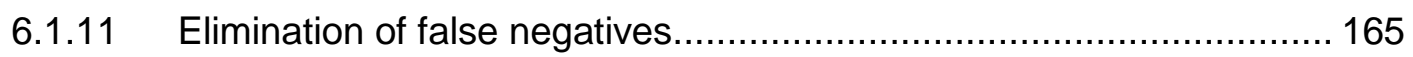

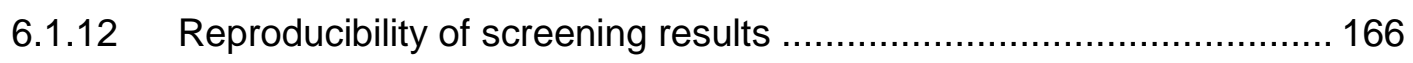

Table 16. Secondary screening and validated hits ......................................... 166

6.1.13 Binding assays and co-immunoprecipitations ................................. 166 
6.1.14 Testing compounds on unrelated targets ...................................... 167

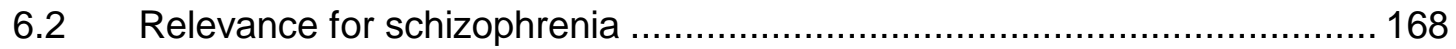

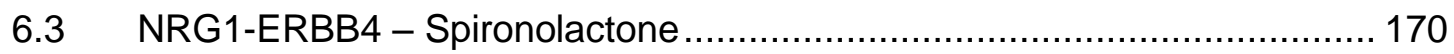

6.3.1 Does Spironolactone physically bind to ERBB4 receptors? ............... 170

6.3.2 Spironolactone derivates and metabolites ....................................... 170

6.3.3 Preliminary validation in a mouse model......................................... 171

$6.4 \quad$ Topotecan shows strong toxic effects ..................................................... 172

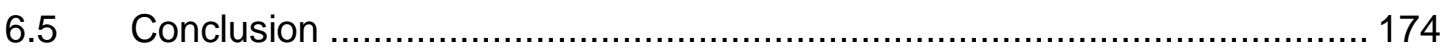

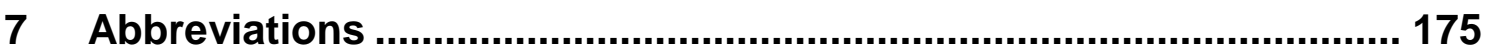

8 Curriculum vitae .................................................................................. 177

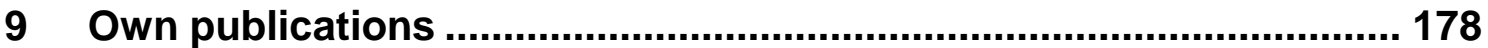

10 Apendix, List of Drugs NCC201/NCC003........................................... 179

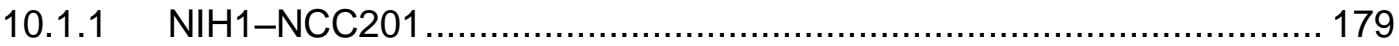

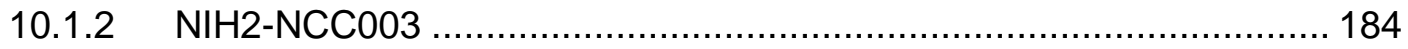

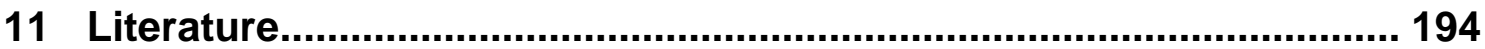




\section{Summary}

Schizophrenia (SZ) is as severe and phenotypically as well as genetically complex neuropsychiatric disorder. Only so-called positive symptoms (e.g. hallucinations and delusions) are currently amenable to treatments with neuroleptics. Negative symptoms (such as anhedonia, social withdraw) and cognitive deficits, however, are not substantially improved by current therapies. An impaired function of the prefrontal cortex has been hypothesised as a potential cause for the latter symptoms. Impaired NRG1-ERBB4 signalling affects cortical circuits formed between inhibitory interneurons and excitatory pyramidal neurons. The corresponding dysbalance of inhibition and excitation is thought to be causative for the cognitive deficits observed in corresponding gain and loss-of-function mouse models. Importantly, genetic association studies have identified NRG1 and ERBB4 as risk factors for SZ. Therefore, NRG1ERBB4 signalling is among the most promising targets for the development of new treatments for SZ.

This thesis describes the design and development of a split TEV-based coculture assay to monitor several steps of the NRG1-ERBB4 signalling cascade in one integrated measurement. The assay displays high sensitivity and robustness and is applicable to high-throughput approaches. In a proof-ofprinciple, the assay was used to screen 727 FDA-approved drugs. Spironolactone was recovered as a major hit, acting as an inhibitor of NRG1ERBB4 signalling and was validated by an extensive set of secondary assays including several technical controls. Moreover, specificity and first mode-ofaction analyses were performed with a panel of assays with NRG and ERBB family members as well as different components of the signalling cascade.

In summary, the assay monitoring proximal aspects of the inter- and intracellular NRG1-ERBB4 signalling cascade was successfully established and a first hit was validated. The assay qualifies for high-throughput screenings with huge libraries of diverse chemical compounds and thus holds a great promise to identify and improve new lead structures to modulate NRG1-ERBB4 signalling. 


\section{Introduction}

\subsection{General characterisation of schizophrenia}

Schizophrenia (SZ) is a severe brain disorder ((Lewis and Lieberman, 2000)(Lewis and Levitt, 2002), a major mental illness and a severely debilitating neuropsychatric disorder (Arnold and Trojanowski, 1996). The term SZ was first introduced in the beginning of the $20^{\text {th }}$ century by Bleuler (Pratt et al., 2012). Kraepelin described the illness as "Dementia praecox"(Kraepelin, 1896).

The diagnostic criteria for schizophrenia are described in the ICD 10 (International Classification of Diseases 10) as well as DSM IV (Diagnostic and Statistical Manual of Mental Disorders). These literal classification schemes are important, since no biological marker of SZ has been identified so far. The classification is based on the categorization of behavioural phenotypes by interviews and are inherent to biases possible by the subjective evaluation of individual clinicians. Nontheless because our understanding of biomarkers based on causal genetic, biochemical or pathophysiological events remains scarce (Pratt et al., 2012) there is no alternative. Moreover, the term disorder is under debate, shifting to disease (Becker, 2005; Berganza et al., 2005; Steurer et al., 2006), taking in account the specific etiology and the discernable pathology of SZ (Tandon et al., 2008b). Further debates, to deconstruct the term SZ itself as it represents an artificial term for different diseases with similar phenotypes are on-going (Tandon et al., 2008a).

The prevalence of SZ ranges between $0.3 \%$ and $2.0 \%$, with an average of $0.7 \%$ in the population of Western Europe. Although occurring worldwide, SZ seems to be independent of culture, political system, or religion (Saha et al., 2005). The precise determination of the number of affected subjects is impossible due to the lack of biomarkers, valid and reliable diagnosis, and precise demarcation between diseased and healthy people (Eaton et al., 2007).

The onset of the disease occurs usually after puberty or during early adulthood (age 15-45) (Fig. 1). Affected men are usually younger than women (average five-seven years earlier) when the disease starts and seem to have a slightly increased risk to suffer from SZ (up to 1,4 fold higher risk) (Aleman et al., 2003; McGrath, 2007). The onset of SZ is usually preceded by a prodromal phase with an average lengths of five years (Klosterkötter, 2008). The prodromal phase often includes the manifestation of cognitive deficits, impairments in social functions, and a drop in job or school related performance (Tandon et al., 2009). 
After the prodromal phase chronic SZ starts mostly by a psychotic episode. The "phenotype" of the disease can further be classified by course (continuous or episodic) onset (acute, progressive or stable), and outcome (mild, severe or recovery). The most abundant type is an episodic course with distinct psychotic episodes.

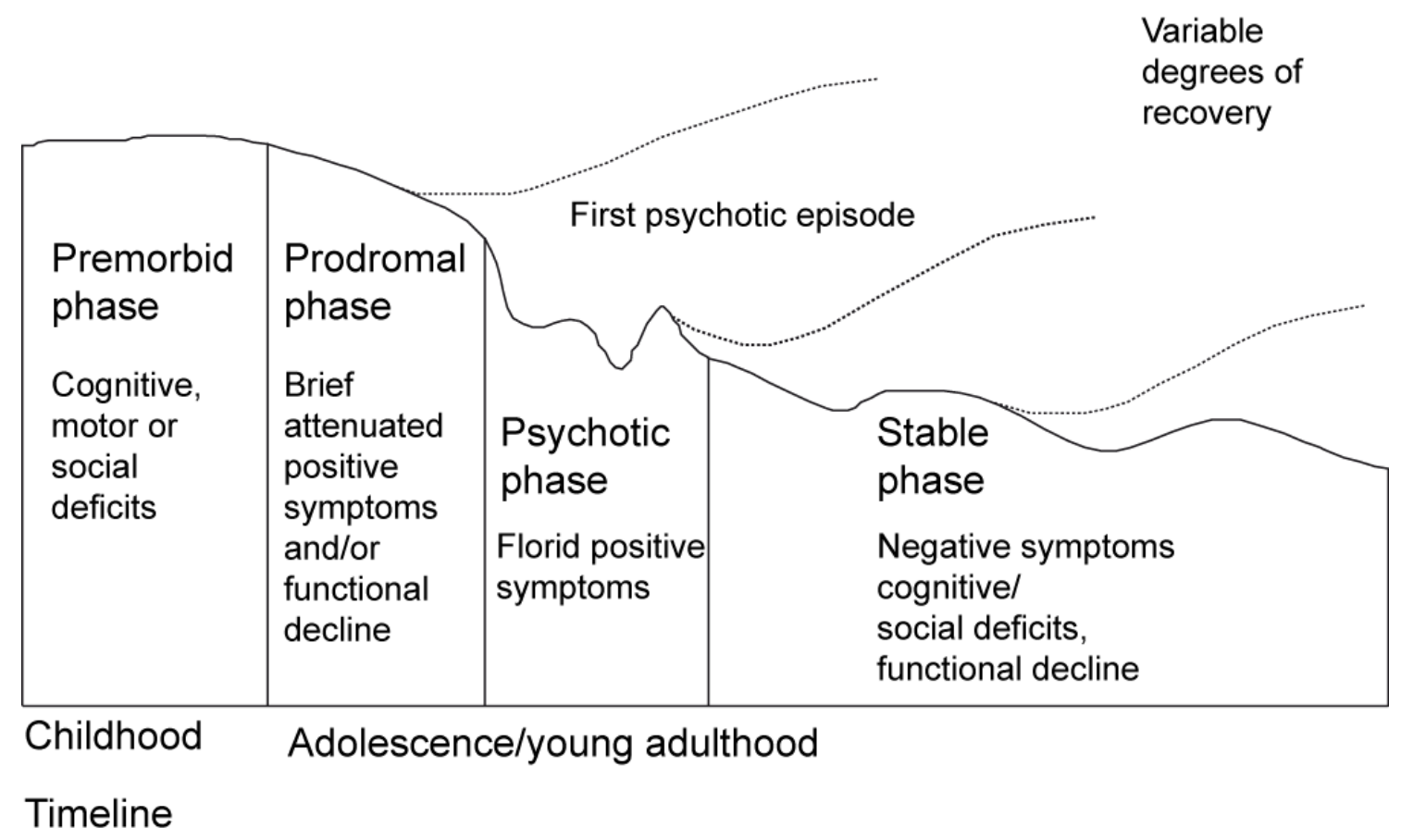

Figure 1: Schematic representation of the course of schizophrenia, modified from (Tandon et al., 2009)

SZ is characterised by three subsets of symptoms: positive symptoms, negative symptoms, and cognitive deficits (Fig. 2). 


\subsection{Positive symptoms}

Positive symptoms are the prominent phenotypic symptoms during an acute psychotic episode where patients may suffer from different sorts of hallucinations (e.g. visual, audio, tactile, olfactory, or gustatory hallucinations), delusions or thought disorders. Patients might suffer upon e.g. hearing of a voice keeping up a running commentary on the persons behaviour or thoughts or experience olfactory hallucinations like the smell of dead people. The patients usually develop more complex delusions (bizarre delusions, delusions of control, organisations) severely altering or modifying the perception of reality. As a part of this, single symptoms are reported, such as the feeling of thought insertions or thought withdrawal, thought echos and thought broadcasting. This leads to feelings of suspiciousness or persecution, extending to attempts of suicide. In contrast, even grandiosity or hostile behaviour is observed depending on the perception content of the delusions. The behaviour of the patients is furthermore impaired by disorganised speech and/or disorganised behaviour, which can be catatonic, but also a complete disorganisation of conceptual thinking. In $75 \%$ of patients these positive symptoms can be reduced by the use of anti-psychotic drugs. At least $25 \%$ of patients do not respond to the treatment at all (Tandon et al., 2010; Tandon et al., 2009; Keshavan et al., 2008; Tandon et al., 2008b; Tandon et al., 2008a).

\subsection{Negative symptoms}

Negative symptoms are not as prominent as positive symptoms. However, negative symptoms can start years before the first psychotic episode and often persist after the decline of the psychotic episode. Patients may suffer from social withdrawal, reduced, flat or inappropriate affect, alogia (poverty of speech), avolition (generalized lack of motivation to perform tasks or undertake activities), emotional withdrawal, and poor rapport. All these symptoms distract the patients to live a normal social life i.e. they may not be integrated in networks of relationships, friends, colleagues, and even medical care personal. In particular solitude has been reported by patients to be one of the major symptoms ruining the quality of their life. The negative symptoms are poorly targeted by classical drugs (Tandon et al., 2009; Tandon et al., 2010).

\subsection{Cognitive deficits}

Furthermore, there is an impact of the disease on the cognitive abilities of the patients. These cognitive deficits are the most debilitating and persistent deficits in SZ. A drop in performance in every-day life regularly accompanies the progress in the development of the negative symptoms, e.g. at school, at the university or in professional life. Cognitive impairments are further impaired with the onset of psychotic episodes. Deficits in working memory, difficulties in 
abstract thinking or stereotyped and inflexible thinking are usually observed. The cognitive deficits remain impaired after fading of the psychotic episode and can worsen with further episodes or over time. The cognitive deficits, as the negative symptoms, do not respond regularly to medication and remain largely resistant to treatment (Arnold and Trojanowski, 1996; Pratt et al., 2012).

Negative and cognitive symptoms are not efficiently targeted by available drugs and are factors which most significantly contribute to worsening of patients individual quality of life. SZ is laying an annual burden of 30 billion $€$ on the European health care systems (33 billlion US dollars in 1999 worldwide (Bayer 1999)) and is among the top ten leading causes of disease related disability in the world (WHO statistics 2001, (Tandon et al., 2008b)).

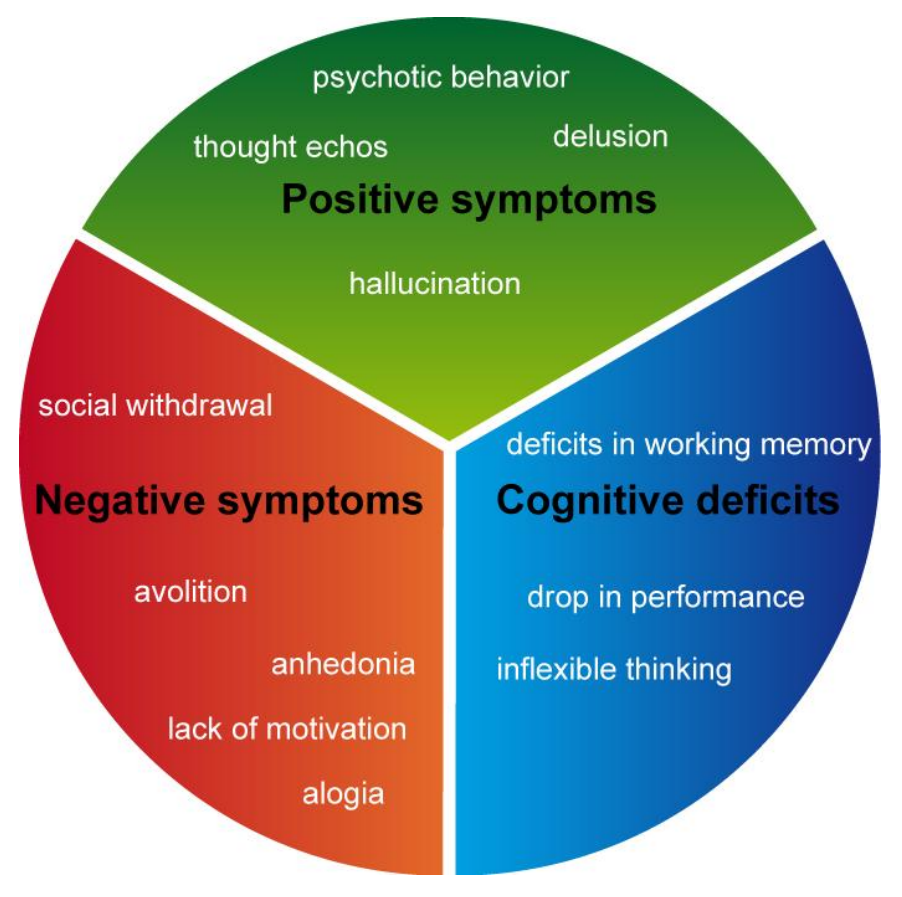

Figure 2: Triade of SZ symptoms: positive symptoms, negative symptoms and cognitive deficits are shown with exemplary phenotypes

\subsection{Comorbidities}

Single symptoms, or even mixtures of the symptoms described above, can also occur in various other mental diseases. For the differential diagnosis of SZ by a psychiatrist, different other illnesses e.g. depression, brain tumour, have to be ruled out as causative for the symptoms or, if occurring in parallel, separately treated. The diagnosis is further complicated by a high rate of psychiatric comorbidity in the patients. The most frequently present psychiatric comorbidities are depression, suicide tendency, substance abuse, sleep disturbances and addiction. 
Diabetes mellitus, autoimmune disorders and cardiac autonomic dysregulation are the most often described non-psychiatric comorbidities. Furthermore, the medications lead often to severe side effects e.g. metabolic syndrome or tiredness, which have to be necessarily addressed in the treatment of SZ as well. In general, the health state of the SZ patients is most likely impaired in multiple ways (Ferentinos and Dikeos, 2012; Kodesh et al., 2012).

\subsection{Residual symptoms}

There is a set of residual symptoms, which occur with an acute psychotic episode and remain present thereafter. This has been described for most negative symptoms like social withdrawal or flat affect, or mild positive symptoms like eccentric behaviour or odd beliefs (de Bartolomeis et al., 2012).

\section{Subtypes of schizophrenia}

For a better diagnosis, SZ is subdivided in different phenotypic subtypes (Tab. 1). The usefulness of this diversion is debatable.

\begin{tabular}{|l|l|}
\hline Type & Description \\
\hline Paranoid (F20.0) & $\begin{array}{l}\text { hostile or threatening auditory hallucinations, } \\
\text { delusions }\end{array}$ \\
\hline Catatonic (F20.2) & $\begin{array}{l}\text { motoric immobility, stuporous catatonia or } \\
\text { exited catatonia / excessive motor activity, } \\
\text { echolalia, echopraxia }\end{array}$ \\
\hline Disorganized (F20.1) & $\begin{array}{l}\text { disorganized speech, disorganized behaviour, } \\
\text { flat or inappropriate affect }\end{array}$ \\
\hline Undifferentiated (F20.3) & $\begin{array}{l}\text { mixture or emerging process of different } \\
\text { subtypes delusions, }\end{array}$ \\
\hline Residual (F20.5) & $\begin{array}{l}\text { Absence of prominent presence of netive } \\
\text { hallucinations etc., presence } \\
\text { symptoms }\end{array}$ \\
\hline
\end{tabular}

Table 1: $\quad$ SZ subtypes with the most prominent behavioural phenotypes.

Depicted are the 5 common subtypes of SZ used, when diagnosed by a psychiatrist. The subtypes are diverse and the borders do not display clear demarcation lines. Modified from (ICD-10) 


\subsection{History of schizophrenia}

The first identifications of mental illnesses and attempts to cure these are unknown but it's history might be as old as human consciousness itself.

Symptoms of a mental disease were first mentioned 3400 years BC in the Book of Heart, an Egyptian papyrus (Okasha, 1999; Okasha, 2001; Mohit, 2001). However, whether these mentions can be linked to $S Z$ is unclear or remains elusive, as this medical term did not exist at that time. Other descriptions, of putative SZ phenotypes, were found in the Indian vedas and in some books of the bible e.g. Nebukadnezar written ca.1400 BC. Skulls with trepanations are sometimes interpreted as early attempts to cure mental diseases (Clower and Finger, 2001).

The Greek philosophers Thales, Phytagoras, Galen, and Hippocrates provided the first theories of the mind and consciousness, and what mental diseases possibly could be at ca. 550 BC. The theories included natural courses and miss-regulations of the four humours. The function of the brain itself was poorly understood (Missios, 2007).

During the dark ages mental illnesses were regarded as evil possessions and no progress in theories could be made. Exorcisms and witch burnings were regular events. In 1357, the Bethlem asylum was founded in London, where dangerous mental diseased patients were simply chained and locked away.

In the $17^{\text {th }}$ century, John Locke $(1632-1704)$ came up with the theory that the brain is empty at birth and is filled in a process of learning by e.g. sensory input. Mental diseases are regarded as a disconnection of the relationship between the environment and the affected person.

In the $18^{\text {th }}$ century Phillippe Pinel $(1745-1826)$ began to unchain the patients in the asylums.

In the $19^{\text {th }}$ century Wilhelm Griesinger $(1818-1868)$ proposed that the brain is the origin / source of the mental diseases, and that these diseases are complex. In addition he shaped the term "unitary psychosis".

Emil Kraeplin (1856-1926) proposed the dichotomy between "dementia praecox" and "bipolar disorder". He also observed the symptoms in longitudinal manner and was convinced that an organic brain disease causing SZ symptoms exists.

In 1897 Alois Alzheimer published the first study on patients having SZ symptoms.

In 1911 Eugen Bleuler (1857-1939) coined the term "schizophrenia" He based it on the Greek words skhizein $=$ to split and phren $=$ mind. 
The first half of the $20^{\text {th }}$ century was dominated by eugenic and social darwinistic pseudo-science, that even lead to mass murder on mentally diseased patients in Germany ("Action T4"). In 1952 the first international congress on neuropathology in Rom concluded: "There is no neuropathology of SZ".

In 1976 a computer tomography (CT) study on schizophrenic brains conducted by Johnstone showed an enlargement of the lateral ventricles. Meanwhile, the development of neuroleptics allowed for the first time to a state of control of the psychotic disease symptoms, which permitted $3 / 4$ of the patients to leave the asylums, e.g. in USA the number of patients in asylums decreased from 600,000 to 150,000 . A wide range of treatment strategies were developed starting from lobotomy, relaxing therapies, lithium, insulin coma, to psychotherapy and psychoactive drugs (Jeste et al., 1985, Ellard, 1987). For further readings see (Russel and Cohn 2012)

\subsection{Morphologic findings in schizophrenia}

The cause of the disease remains unknown. Although the behavioural phenotype is strong, the morphological abnormalities in the brain are more subtle and elusive. SZ is nowadays considered as a multifactorial disease including multiple genetic and environmental components, potentially modulated by epigenetic- risk factors. (Tandon et al., 2008b; Tandon et al., 2009). In detail the following findings are validated and discussed.

Magnetic resonance imaging (MRI) showed in first episode SZ patients a reduction of the total brain volume, an enlargement of the ventricles and a reduction of the volume of the hippocampus. Analysis of high-risk individuals shows growing evidence for subtle abnormalities of hippocampal and ventricular volume in close relatives of SZ patients (Whalley et al., 2012).

Only a few degenerative processes are described for SZ (Meyer-Lindenberg, 2011). A dynamic wave of accelerated grey matter loss has been published (Honea et al., 2008). The loss is most prominent in the early phases of SZ. First, the parietal regions are affected, afterwards the temporal lobes, and subsequently changes in dorsolateral prefrontal cortices and superior temporal gyrii are observed. The yearly grey matter loss in SZ patients has a rate of $0,5 \%$ compared to $0,2 \%$ in healthy individuals (Rund, 2009).

Medical findings in SZ patients using EEG recordings are not specific for SZ. Deficits in sensory-motor gating measured as pre-pulse inhibition are observed. However, again they are not specific for SZ and are present in other psychiatric diseases as well. Moreover, SZ patients display impairments of synchronisation of different brain areas (Fig 12). A disturbance in sensory gating of the p50 
wave is often found in SZ patients. A failure in the corollary discharge system is hypothesized as causative for hallucinations (Ishii et al., 2012).

Autopsies taken from post mortem brains of SZ patients show a decrease in brain weight and brain length, a decrease of the volume of cerebral hemispheres, and an enlargement of the ventricles (Kleinman et al., 2011).

In vivo neuroimaging studies revealed alterations in the ventricular system. SZ brains display abnormalities in the cavum septi pellucidi, an enlargement of the lateral ventricles, and the third ventricle. The grey matter volume is decreased in the prefrontal cortex. A decrease in the thalamus, temporal lobe structures, medial temporal lobe (hippocampus/amygdala), and the superior temporal gyrus is described. The white matter integrity is decreased in all four cortical lobes (Fornito et al., 2012).

At the cellular level microscopic studies of the hippocampus and prefrontal cortex have shown no overall change in neuron numbers, but a size reduction of the mean neuronal soma, and a higher package density of neurons in the prefrontal cortex. The thalamus displays a lower number of neurons in the mediodorsal, anteroventral, and anteromedial nuclei. The entorhinal cortex is characterised by a poorly developed layer II and a disturbance in the layer structure (Arnold and Trojanowski, 1996).

Other theories in the field link findings of auto immune reactions and micro glia malfunctions to the disease (Monji et al., 2009). For further information, see (Hanisch and Kettenmann, 2007; Chen et al., 2010).

Fast progress in individualised medicine enabled derivation of human induced pluripotent stem cells (hiPSC) from patients. The re-programming of patient fibroblasts infected with lentiviruses containing genes for the factors Oct4, SOX2, KLF4, CMYC, and Lin 28, lead to the growth and differentiation of disorder specific neurons. The SZ hiPSC display diminished neuronal connectivity and reduced expression of PSD95 and glutamate receptors. Moreover, alterations in the expression of components of cyclic AMP (cAMP) and the WNT signalling pathways have been reported. Furthermore, the neurons obtained from hiPSC of SZ patients respond to antipsychotic treatment. Experiments on hiPSC of SZ patients give for the first time an unique opportunity of investigating $S Z$ in vitro which offers a tremendous potential to find new personalised treatments for SZ patients ("SZ in a box") (Brennand et al., 2011).

\subsection{Genetic studies}

Adoption studies, twin studies, family-based studies, and population studies showed an increased risk to develop SZ depending on the degree of kinship 
(Sullivan et al., 2003). The average prevalence in any society in any country in the world to develop the illness is $0,7 \%$ (Saha et al., 2005). For children of two SZ-positive parents, and for gene identical monozygotic twin where the other suffers from SZ the overall risk is increased to $40 \%-50 \%$ (Ayhan et al., 2009). The overall genetic impact on the development of SZ is estimated $70 \%-80 \%$ (Sullivan et al., 2003; Wexler and Geschwind, 2011). Family-based studies identified a gene that is disrupted and plays a role in the disease (disrupted in SZ: DISC1, Scottish family). However, genome-wide genetic association studies (GWAS) failed to identify a single gene or a specific subset of genes being causative for the disease. Nevertheless, it was possible to identify different single nucleotide polymorphisms (SNPs) and rare copy number variations (CNVs) in genes, which seem to be associated with brain changes leading to higher vulnerability (Sebat et al., 2009). The precise contribution of each genetic alteration remains unclear and combinatorial effects of different risk alleles are debated as effective cause (Allen et al., 2008; Jia et al., 2010). In addition, a current model suggests that a set of complex disease pathways, where various different alleles lead in different combinations to disparate neuropsychiatric disorders (Figure 3) (SZ, bipolar and unipolar depression, mental retardation, seizures and autism, no clear demarcation lines) (Karam et al., 2010). This model also emphasizes that the discovery of a single pharmacological intervention treating all symptoms at once is highly unlikely. In contrast, newly developed drugs probably target similar symptoms in different neuropsychiatric disorders (Karam et al., 2010).

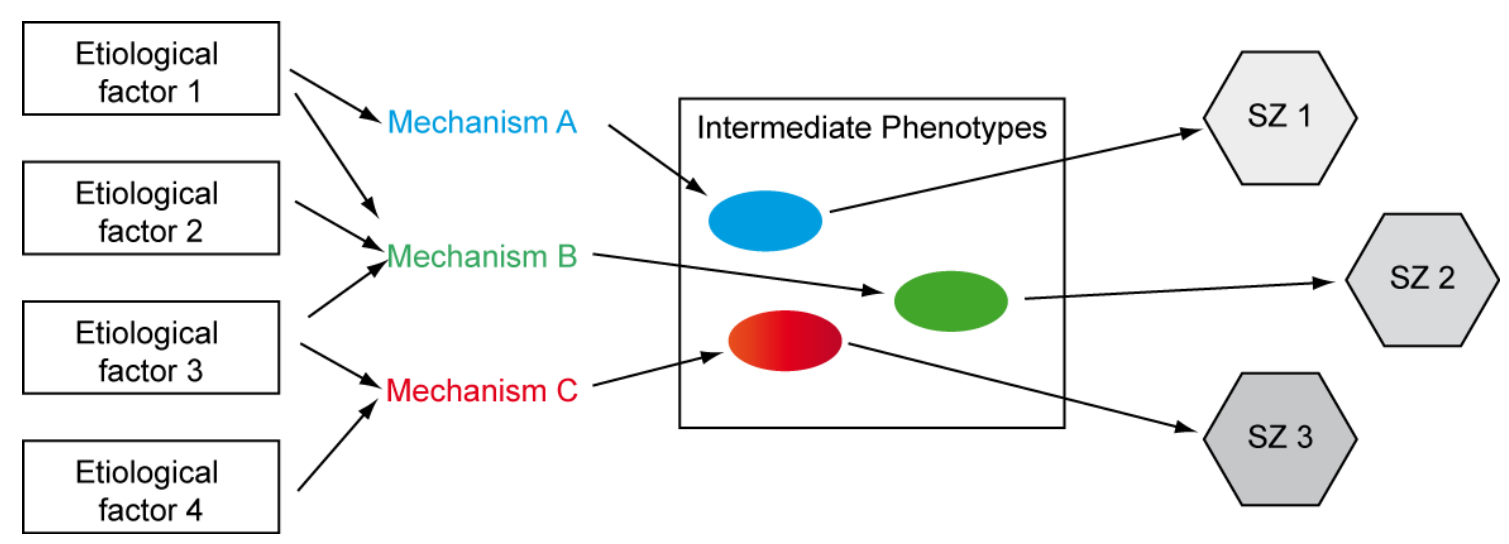

Figure 3: Model for SZ: The endpoint result is different SZ types (Tandon et al. 2008)

Some of the most frequent $\mathrm{SZ}$ susceptibility genes are:

\subsubsection{DISC1}

The DISC1 (disrupted in schizophrenia 1) gene was linked to the disease by St Clair in 1968, when he discovered a chromosomal translocation from 
chromosome chr.11 to chr1 in a Scottish boy. The translocation truncated a single gene, DISC1. The truncation was present in a half of the boy's family that was diagnosed with severe mental diseases. Later linkage of DISC1 to SZ was confirmed in Finish, Caucasian and Asian populations.

DISC1 is a large scaffolding protein $(93 \mathrm{kDa})$, prominently expressed in the human hippocampus. It interacts with MAP1a, GSK-3 $\beta$, PDE4, FEZ1, and NDEL1. DISC1 is also downstream of the NRG1-ERBB4 signalling cascade (St Clair et al., 1990; Millar et al., 2000; Kang et al., 2011; Wexler and Geschwind, 2011).

\subsubsection{NRG1-ERBB4}

The first findings on impaired NRG1-ERBB4 (Neuregulin1 - receptor tyrosineprotein kinase v-erb-a erythroblastic leukemia viral oncogene homolog 4) signalling were made by Stefansson and colleagues in the genome wide association studies on an Icelandic population (Stefansson et al., 2002). The identified SNP SNP8NRG243177 or 'icelandic' haplotype (HAPice) has been associated with several schizophrenia-like phenotypes, e.g impaired frontal and temporal lobe activation, deficits in cognitive functions and a predisposition for the development of psychotic symptoms (Hall et al., 2006).

Until 2009, more than 80 SNPs in the NRG1-ERBB4 receptor-ligand pair have been associated with SZ (Sebat et al., 2009). Patients with alterations in the $\mathrm{Nrg} 1$ gene display endophenotypes like decrease in pre-puls inhibition (Hong et al., 2008), reduced integrity of the white matter (Mclntosh et al., 2008; Winterer et al., 2008), hypofrontality, cognitive dysfunction and decreased IQ (Hall et al., 2006). Most genetic polymorphisms of the $\mathrm{Nrg} 1$ gene are found in the 5' region, the regulatory element of the $\mathrm{Nrg} 1$ gene (Harrison 2006). It is speculated that this alterations contribute to alterations in gene function and expression. A meta-analysis of up to 24 different studies in diverse ethnic groups successfully confirmed these findings (Munafò et al., 2006; Li et al., 2004; Li et al., 2006).

The Nrg1 gene is a key player of numerous processes implicated and discussed in the development of SZ. NRG1 contributes to myelination, development of glial cells, migration of radial glial cells during cortical development, neuronal plasticity modulated by NMDA receptor function, development of GABAergic interneurons, and expression of dopamine and serotonin receptors and monoamine transporters (Mei and Xiong, 2008). Furthermore, NRG1 modulates the formation of dendritic spines (Chen et al., 2008), and gamma oscillations in the hippocampus (Fisahn et al., 2009). Mice heterozygous for Nrg1 show schizophrenia-related phenotypes resembling symptoms observed in SZ patients (see below). 
NRG1 has been shown to increase the expression of $\alpha 7$-nicotinic receptors (Hancock et al., 2008). It is postulated that alterations in NRG1 mediated signalling changes the nicotinic receptor profile in presynaptic inputs, leading to deficits in pre-puls inhibition by altering glutamatergic transmission from the ventral hippocampus to the nucleus accumbens (Karam et al., 2010).

Additionally, the NRG1-ERBB4 signalling pathway is a major input for various pathways, involving PI3-kinases, PTPRZ1-RTP $\beta$-phosphatase, BACE secretase, and the afore mentioned DISC1. Many of these are hubs at information processing, and may be suitable as future drug targets (Jaaro-Peled et al., 2009; Höistad et al., 2009).

\subsection{Environmental factors}

Many studies show the importance of environmental factors in onset, cause and severity of the disease. The concordance for SZ in monozygotic twins is only $50 \%$. Therefore genes cannot be the only cause for the disease. The most prominent environmental factors contributing to development of SZ are antenatal or obstetric complications (hypoxia, maternal infections, maternal drug - intake) (Nicodemus et al., 2008)), migration (first reported by Odegard 1932, (Cantor-Graae and Selten, 2005), urbanicity (Kirkbride et al., 2006), parental age, malnutrition and psychotrauma (Keshavan et al., 2008). All these factors seem to create psychosocial stress. Just like the genetic factors, environmental factors alone fail to explain the disease (Fig. 3). The combination of genetic and environmental factors leads to the "Two Hit Hypothesis".

\subsection{Two hit hypothesis}

The "Two Hit Hypothesis" of SZ integrates the genetic and environmental observations. The combination of one, or of different genetic defects, either inherited, or de novo mutated, generates a state of vulnerability e.g. a deficient neuronal network. Stressful environmental effects like viral infections, birth complications, or social stress as a second hit can subsequently trigger the outbreak of $S Z$ by modulating the expression or function of the defective genes or proteins (Bayer et al., 1999; Maynard et al., 2001).

The proposal of a "Three Hit Hypothesis", additionally taking in account drug effects like cannabinol (THC), as an additional player to genes and environment interaction, is under debate (Vigano et al., 2009). 


\subsection{Findings in animal models of schizophrenia}

Animal models of diseases, reflecting the genetic findings, are highly desired and deliver valuable information about the disease. The problem with animal models of complex diseases, which cannot be associated with a single gene, or that are multifactorial disorders like SZ, is to generate a valid model for a disease.

A valid animal model has to meet the following criteria:

- Face validity (animal model shows the symptoms of the disease)

- Construct validity (animal model has the same mutation or genetic alteration underlying the mechanisms of disease aetiology)

- Predictive validity (animal model responds to treatments similar as patient)

As the precise cause and the multi factorial interaction remains unknown, construct validity of the different animal models in SZ is not possible. Consistent with the complexity of the disease and spectrum of symptoms, genetic research has led to the development of several animals models that are thought to mimic parts of the disease. They show a broad spectrum of endophenotypes similar to observations of a few positive symptoms, negative symptoms, and cognitive deficits in SZ but "the schizophrenia model" will not be possible to generate (Kellendonk et al., 2009).

The first mouse models in the field were drug induced 'psychotic' models. Mice were simply treated with Amphetamine or Phencyclidine until they show psychotic behaviour (such as motor hyperactivity) and were subsequently treated with the newlz developed putative drugs (Javitt and Zukin, 1991).

Amphetamine leads to an over activation of the dopaminergic system and Phencyclidine leads to an over activation of the glutamatergic system. The Dopamine- and Glutamate-Hypotheses sprouted from these observations. Drugs could be tested afterward with these models which lead e.g. to the discovery of antipsychotic drugs like Haloperidol (1958), which is still used to treat psychotic episodes in SZ patients. However, these setup of drug discovery remains serendipitous (Pratt et al., 2012).

\subsubsection{Dopamine hypothesis of schizophrenia}

Dopamine belongs to the family of monoamines and acts as neurotransmitter or neuromodulator. It is recognised via G-protein coupled receptors, localised in the prefrontal cortex, nucleus acumbens, and the striatum. Decreased Dopamin in the prefrontal cortex and increased dopamine in the mesolimbic pathwayarer key findings for SZ (Goldman-Rakic et al., 2004) The classic dopamine 
hypothesis postulates that the drug class of neuroleptics (e.g. Droperidol) act as dopamine receptor antagonists (van Rossum, 1966; van Rossum, 1967). There is a correlation between affinity of these antipsychotic drugs to the Dopamine D2 receptor subtype and the clinical efficiency in countering hallucinations and delusions(Seeman and Lee, 1975). They are hypothesized to block dopamine receptors in the mesolimbic dopaminergic system. This leads to the alleviation of the positive i.e. psychotic symptoms. Unfortunately, side effects resulting from dopamine blockade in the nigrostriatal and hypothalamic-pituitary systems, phenotypically similar to Parkinson's disease, hyperprolactinemia, sedation and hypertension can be observed (Pratt et al., 2012, Vigano et al., 2009).

In 1991, a modified dopamine hypothesis was postulated (Davis et al., 1991, Biedermann and Fleischhacker, 2009) .Cortical hypoactivity, leads to negative symptoms, therefore neuroleptics show no effect. It is based on the observation that humans with frontal lobe brain damages or lobotomy show symptoms similar to SZ negative symptoms. Subcortical hyperactivity, leads to the positive symptoms, which are partially reduced by neuroleptics. Only the D2 receptor subclass, localized in the striatum (subcortical) is targeted by all relevant antipsychotics (Sanyal and Van Tol, 1997).

\subsection{NRG1-ERBB4 animal models}

The finding of impairments in the NRG1-ERBB4 system by GWAS, led to an increased interest in the analysis of different genetically manipulated $\mathrm{Nrg} 1$ and Erbb4 mice to study neuronal and behavioural phenotypes.

Mice that are homozygous null for Erbb4 are dying at postnatal day (P) 10,5 11 because of developmental heart failure (Tidcombe et al., 2003). Heart rescued mice (to prevent developmental death) show defects in neuronal architecture.

Heterozygous knock-out (KO) mice of Nrg1 show hyperactivity and pre-pulse inhibition deficits, one of endophenotypes of SZ.

Nrg1 overexpression (Stefansson et al., 2002), altered social behaviour and increased anxiety (Desbonnet et al., 2009), memory deficits, reduced inhibitory interneuron numbers and increased ventricular volume (Chen et al., 2008). These findings resemble symptoms observed in SZ patients.

Nrg1-typelll overexpressing mouse models and the ErbB4 heterozygous mouse model could be used to validate effects of Nrg1-ERBB4 mediating drugs.

The following table summarizes the phenotypes obtained with Nrg 1/Erbb mouse models: 


\begin{tabular}{|c|c|c|}
\hline \multicolumn{2}{|c|}{ Mouse-model } & \multirow{2}{*}{$\begin{array}{l}\text { Phenotype } \\
\text { Increased locomotor activity, non-significant trend } \\
\text { towards decreased pre-pulse inhibition, decreased } \\
\text { context depending fear learning (Kato et al., 2010). } \\
\text { reduced GABAergic (parvalbumin) and myelination } \\
\text { marker in the frontal cortex (Kato et al, 2008). } \\
\text { Alterations in hippocampus. Hypermyelination and } \\
\text { ataxia for high gene dosage (Brinkmann et al., 2008) }\end{array}$} \\
\hline Nrg1.I & Overexpression & \\
\hline & Heterozygous KO & $\begin{array}{l}\text { Behavioural phenotype linked to SZ phenotype } \\
\text { (Stefansson et al., 2002) Fewer functional NMDA } \\
\text { receptors, react to clozapine (Bjarnadottir et al., 2007) }\end{array}$ \\
\hline & Homozygous KO & $\begin{array}{l}\text { Die during embryogenesis, heart malformations } \\
\text { (Meyer and Birchmeier, 1995) }\end{array}$ \\
\hline \multirow[t]{3}{*}{ Nrg1.III } & Overexpression & $\begin{array}{l}\text { Hypermyelination (Velanac et al., 2012; Michailov et } \\
\text { al., 2004) ataxia for high gene dosage (Brinkmann et } \\
\text { al., 2008) }\end{array}$ \\
\hline & Heterozygous KO & $\begin{array}{l}\text { Poor ensheathment of sensory neurons, } \\
\text { disproportionately unmyelinated or hypomyelinated } \\
\text { nerve fibres. Failure in myelination (Taveggia et al., } \\
\text { 2005; Taveggia et al., 2008). }\end{array}$ \\
\hline & Homozygous KO & Not separated from Nrg1.I \\
\hline \multirow[t]{4}{*}{ ErbB4 } & Overexpression & $\begin{array}{l}\text { Generation and characterization of GAD67-ErbB4 } \\
\text { transgenic mice. (Work in progress Lin) }\end{array}$ \\
\hline & Heterozygous KO & $\begin{array}{l}\text { Behavioural phenotype linked to SZ phenotype } \\
\text { (Stefansson et al., 2002) }\end{array}$ \\
\hline & Homozygous KO & $\begin{array}{l}\text { Lethal at embryonic day (E) 10,5-11, failure in heart } \\
\text { development (Tidcombe et al., 2003). }\end{array}$ \\
\hline & $\begin{array}{l}\text { Homozygous KO heart } \\
\text { rescue phenotype }\end{array}$ & $\begin{array}{l}\text { Defects in cranial neural crest cell migration, aberrant } \\
\text { cranial nerve architecture, increased number of large } \\
\text { interneurons, lactation deficits. Lack of STAT5A } \\
\text { phosphorylation (Liu et al., 1997, Fried et al., 2002). } \\
\text { Altered populations of hippocampal interneurons } \\
\text { (Neddens and Buonanno, 2010). }\end{array}$ \\
\hline
\end{tabular}

Table 2. Shown are the effects of alterations in the Nrg1-ErbB4 signalling system in mouse animal models. 
Several studies combined genetic modified animal models of SZ with environmental factors like e.g. stress (isolation stress in single caging, psychosocial stress by social defeat or maternal separation etc.). This is thought to mimic the "Two Hit" hypothesis of SZ in the respective animal model.

$\mathrm{SZ}$ is a human specific disorder diagnosed by a psychiatrist and obviously cannot be exactly matched in any animal model. Nonetheless, several mouse models display behavioural alterations resembling certain (endo)phenotypes of SZ (Tab. 3). The construct validity underlying these phenotypes, especially regarding modelling of positive symptoms of $S Z$, is often under debate.

\begin{tabular}{|c|c|c|}
\hline Domain & Schizophrenia in humans & Rodent phenotype \\
\hline \multirow{3}{*}{$\begin{array}{l}\text { Positive } \\
\text { symptoms }\end{array}$} & Psychomotor agitation & Hyperlocomotion in open field \\
\hline & Catatonia & Catatonia like state in bar test \\
\hline & $\begin{array}{l}\text { Hallucination, delusion, thought } \\
\text { disorders }\end{array}$ & Lack of readouts \\
\hline \multirow{5}{*}{$\begin{array}{l}\text { Negative } \\
\text { symptoms }\end{array}$} & \multirow[t]{2}{*}{ Social withdrawal } & Decrease in social interaction \\
\hline & & Decrease in nest building ability \\
\hline & $\begin{array}{l}\text { Restricted interest, stereotypic } \\
\text { behaviour }\end{array}$ & Repetitive behaviour \\
\hline & Affect flattening & Lack of reliable readouts \\
\hline & Anhedonia & Decrease in sucrose preference \\
\hline \multirow{7}{*}{$\begin{array}{l}\text { Cognitive } \\
\text { symptoms }\end{array}$} & \multirow[t]{3}{*}{ Working memory deficits } & Impaired performance in T-maze \\
\hline & & Impaired performance in 8-arm maze \\
\hline & & $\begin{array}{l}\text { Decreased spontaneous alternation } \\
\text { in Y-maze }\end{array}$ \\
\hline & \multirow[t]{3}{*}{ Deficit of attention } & $\begin{array}{l}\text { Decrease sustained attention in } \\
5 \text { CSRTT }\end{array}$ \\
\hline & & Decreased set shifting ability \\
\hline & & $\begin{array}{l}\text { Decreased spatial reversal or } \\
\text { reversal of discriminant operant } \\
\text { learning }\end{array}$ \\
\hline & Disturbance of sensorimotor gating & Diminished pre-pulse inhibition \\
\hline
\end{tabular}

Table 3. Tests available for assessment of SZ-related phenotypes in rodent models 


\subsection{NRG1-ERBB4: from proteins to network analysis}

\subsubsection{Why addressing the NRG1-ERBB4 signalling system?}

As described above, gene defects play the major part in the development of the vulnerability to SZ. Nonetheless, available studies fail to link the disease to a single gene or to a precise subset of genes. Therefore, the assumption that SZ is a generic term or a superordinate concept of different diseases ending up in a similar set of similar or slightly different phenotypes is presumable. If there is not "one schizophrenia" existing with one causative SZ gene, I assume that newly developed drugs, for a determined subset of patients are the auspicious novelties. The NRG1-ERBB4 signalling network seems to be one of the most promising and best accessible SZ key players to address in the development of new drugs. In detail the following subsets of information on NRG1-ERBB4 are available.

\subsubsection{Protein-protein-interactions and NRG1-ERBB4 signalling}

Specific protein-protein-interactions (PPI) in cells are the basic units of signalling cascades. Most cellular processes are regulated through signalling cascades or more complex structures of interlinked cascades named signalling networks or pathways (Neddens and Buonanno, 2010). Signal processing needs different regulation of information. This leads to diversification, amplification, diminution or alteration of the signal and interexchange between different cascades. The endpoints of signalling cascades are changes in expression levels of the cellular proteins thereby inducing apoptosis, proliferation, growth or other changes in cellular behaviour.

Of crucial importance for an inter-cellular communication is the reception of signals from other cells. Signals are transferred from the outside to the inside through a ligand representing the stimulus activating specific transmembrane receptors. Once activated, receptors recruit adaptor molecules and activate signalling cascades forwarding information through the cytosol to the nucleus. In the cytosol further processing takes place and different signal cascades are interlinked. In the nucleus gene activity is modulated according to the processed information, causing on and off switching of genes or modulating the gene expression (Taniguchi et al., 2006). A plurality of subsequent processes for example protein turnover and stress reactions to low oxygen levels are important parts of signal processing (Fishman and Porter, 2005).

The overall architecture of the NRG1-ERBB signalling system is organised in a bowtie or hourglass like structure (Fig. 4). A multitude of ligands binds to corresponding subtypes of ERBB receptors, thereby activating a handful of adapters and then initialising various cascades to deliver signals to the nucleus (Yarden and Sliwkowski, 2001; Oda et al., 2005). Drugs and pharmacological 
substances can act through binding of receptors outside the cells, or by inhibiting the functionality of enzymes, or alter the structure of proteins and protein complexes (Oda et al., 2005; Yarden and Pines, 2012).

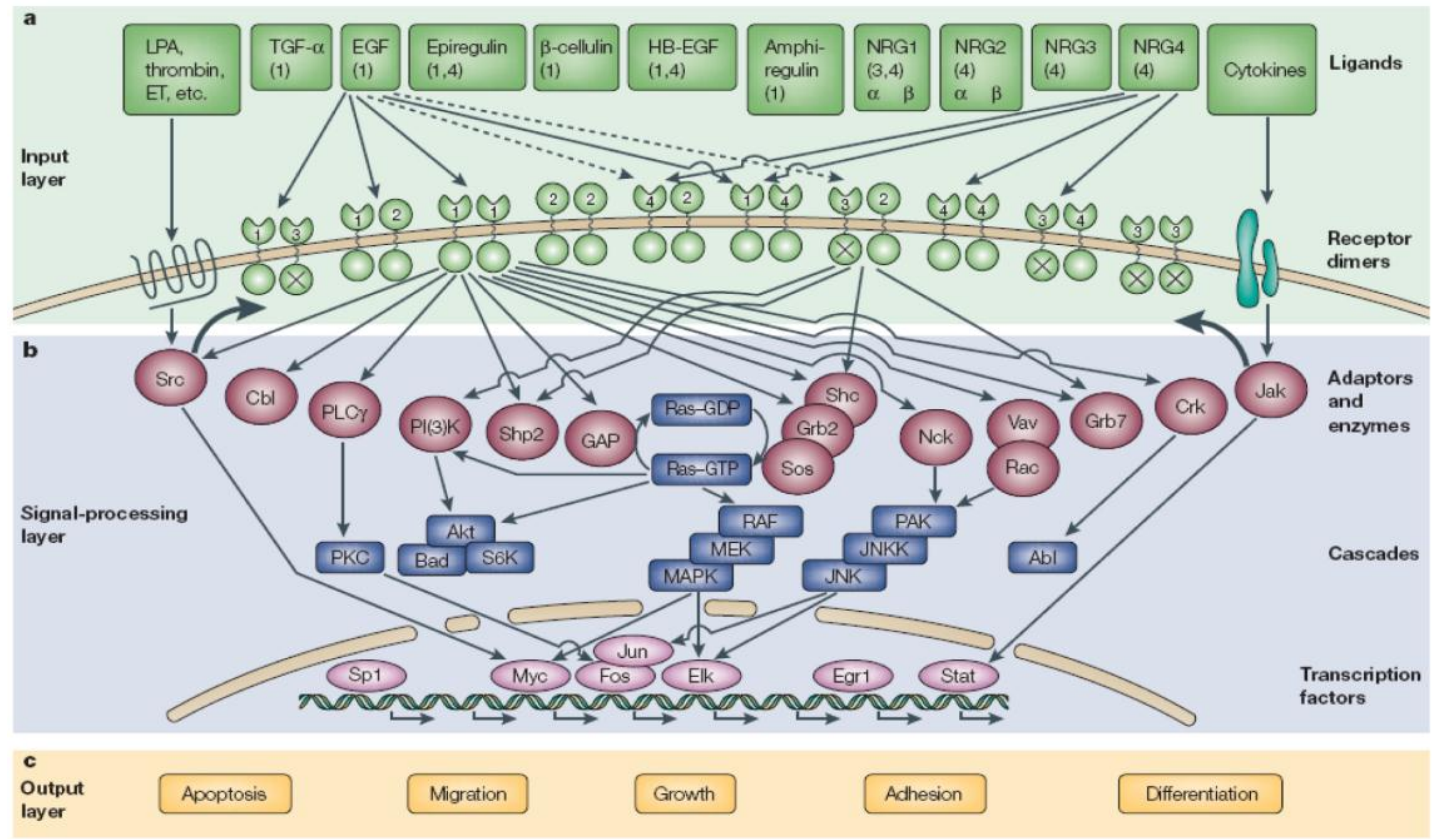

Figure 4: Bowtie structure of the ERBB signalling network (Yarden and Sliwkowski 2001)

\subsubsection{Neuregulin1}

Neuregulins (NRGs) are signalling molecules or ligands that bind to specific receptor tyrosine kinases (RTKs) i.e. ERBB3 and ERBB4. The Neuregulin family consist of four genes named Nrg1-Nrg4. The best characterised ligand is Neuregulin1 (NRG1). More than 31 isoforms of this ligand are described (Falls, 2003). The effect of the NRG1 ligand depends on the cell type, tissue context and activated receptors. NRG1 acts e.g. as differentiation factor in the peripheral nervous system (PNS) (Adlkofer and Lai, 2000) and regulates myelin sheet thickness in axon-Schwann cell interactions (Michailov et al., 2004). In the central nervous system (CNS) NRG1 promotes synaptogenesis (Kwon et al., 2008; Ozaki et al., 1997).

Historically, NRG1 was associated with various biological functions. First, it was named Heregulin/neu differentiation factor (HRG/NDF) that activates HER/neuoncogenes or glial growth factors that promote proliferation of Schwann cells (Grossmann et al., 2009; Brockes et al., 1980). Furthermore, NRG1 was found to increase the expression of acetylcholine receptors in muscle cells (Falls, 2003). 


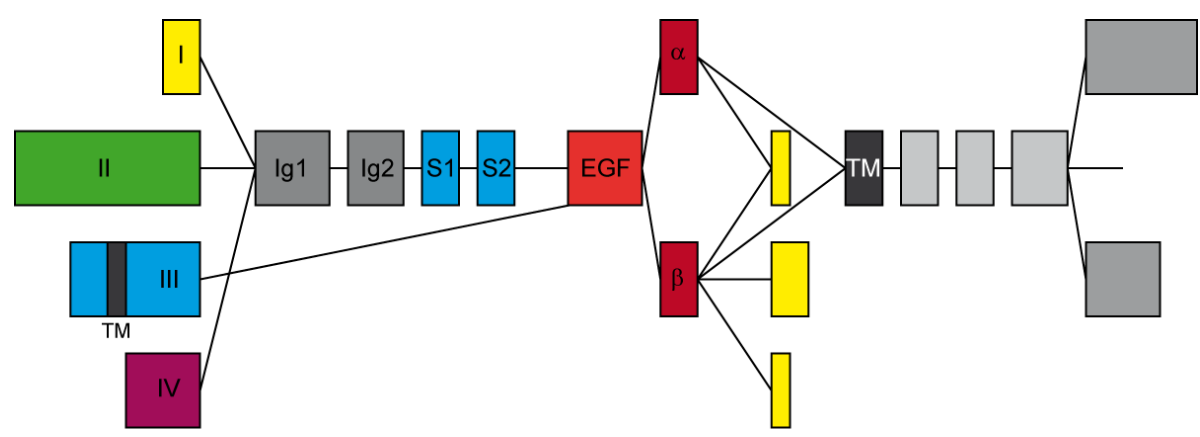

Figure 5: Splice variants of Nrg1 (modified Mei Xlong 2008)

The three most abundant splice isoforms of NRG1 in mice are Nrg1-typel- $\beta 1 \mathrm{a}$, Nrg1-typell- $\beta 1 \mathrm{a}$ and Nrg1-typelll- $\beta 1$ a (Fig. 5). All isoforms harbour a C-terminal transmembrane domain, but differ in the $\mathrm{N}$-terminus. Type I and type II harbour an extracellularly localised immunoglobulin like (lg-like) domain. In contrast, type III contains a cysteine-rich domain (CRD) with an additional transmembrane domain. The transmembrane isoforms of NRG1 are cell type dependently processed by a set of metalloproteases, e.g. TNFalpha-converting enzyme (TACE) or beta-site APP cleaving enzymes (BACE). When cleaved, the NRG1 intracellular domain can back signal to the nucleus and the extracellular domain acts as a paracrine (type I-II) or as juxtacrine signal (type III) (Mei and Xiong, 2008).

NRG1 binding activates ERBB3 and ERBB4 receptors by inducing homo- and hetero-dimerisation of ERBB1-4 receptors, in particular NRG1 can activate ERBB1-3, 1-4, 2-3, 2-4, 3-3, 3-4, 4-4 receptor pairs (Mei and Xiong, 2008). It is postulated that a single ligand is sufficient to activate the dimers (Liu et al., 2012).

The most prominent bioactive part of all Nrg1 isoforms is the EGF like domain. The EGF like domain is 55 amino acids (AA) long (Fig. 6). A 62 AA stretch, resembling NRG1 AA Thr176-Lys238, including the EGF like domain, is commercially available via different manufactores. Thiss domain is overexpressed in bacteria and afterwards purified. It is highly active and stimulates ERBB3 and ERBB4 receptors similar to native NRGs (Liu et al., 2012). 
$\begin{array}{rrrrrr}10 & 20 & 30 & 40 & 50 & 60\end{array}$

$\begin{array}{rrrrrr}70 & 80 & 90 & 100 & 110 & 120 \\ \text { KW } & \text { FKNGNELNRK } & \text { NKPQNIKIQK } & \text { KPGKSELRIN } & \text { KASLADSGEY } & \text { MCKVISKLGN }\end{array}$

$\begin{array}{rrrrrr}130 & 140 & 150 & 160 & 170 & 180 \\ \text { DSASANITIV } & \text { ESNEIITGMP } & \text { ASTEGAYVSS ESPIRISVST } & \text { EGANTSSSTS } & \text { TSTTGTSHLV }\end{array}$

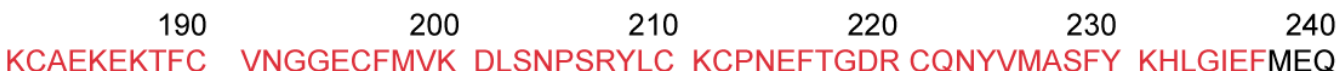

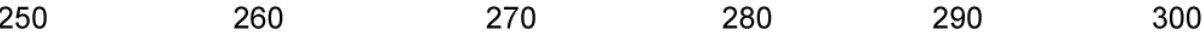

KRVLTITGIC IALLVVGIMC VVAYCKTKKQ RKKLHDRLRQ SLRSERNNMM NIANGPHHPN

Figure 6: Amino acid sequence of NRG1. Complete precursor chain of NRG1-beta, the EGF like domain is highlighted in red. Modified from Reprokin.

\subsubsection{ERBB receptor tyrosine kinase family}

In humans there are four different ERBB receptors described, named EGFR, ERBB2, ERBB3, and ERBB4. Some of them have different splice variants.

ERBB receptors recognise specific signalling molecules e.g. NRGs or EGF and react by forming homo or hetero dimers. The receptor-complexes promote the signal by cross-phosporylation by tyrosine kinase activities and subsequent recruitment of phosphor-adaptor molecules, that than lead to the activation of further signalling cascades.

\begin{tabular}{|l|l|l|l|}
\hline ERBB1 & EGFR & HER1 & mErbB1 \\
\hline ERBB2 & Neu & HER2 & mErbB2 \\
\hline ERBB3 & & HER3 & mErbB3 \\
\hline ERBB4 & & HER4 & mErbB4 \\
\hline
\end{tabular}

Table 4. Names and synonyms for ERBB receptors. HER: human epidermal growth factor receptor.

m: murine, (Citri and Yarden, 2006; Yarden and Sliwkowski, 2001)

All ERBB receptors are glycosylated type I trans-membrane proteins characterised by four extracellular domains (I-IV) and one intracellular tyrosine kinase domain (Fig. 7 and 8). NRG ligands bind to the extracellular domains I (L1) and III (L2). The cysteine rich domains II and IV take part in the receptor dimerization (Burgess et al., 2003). After dimerization, the receptors phosphorylate each other, shift to the trans state and activate signalling cascades via adaptor recruitment. All ERBB receptors are capable of forming homo- or heterodimers with each other (Yarden and Pines, 2012). 
ERBB2 lacks the ability to bind to ligands (Marmor et al., 2004, Yarden and Sliwkowski, 2001). Furthermore, the receptor shows high auto-phosphorylation levels (Lonardo et al., 1990) which leads to its constitutive activity (Di Fiore et al., 1990). ERBB2 is a classical breast cancer candidate being the first receptor ever targeted utilising an antibody (Trastuzumab, trade name Herceptin 1998) in an anti-cancer therapy (Hudis, 2007). The preferred binding partners for ERBB2 are ERBB3 and ERBB4 (Tzahar et al., 1996).

ERBB3 has no active tyrosine kinase domain. The receptor can bind to adapter molecules but its hetero-dimerisation is necessary for promoting signals (Carraway et al., 1994).

ERBB4 is the most similar receptor compared to EGFR (Plowman et al., 1993). At least 4 different isoforms of the receptor are known: ERBB4-JM-a-CYT-1, ERBB4-JM-a-CYT-2, ERBB4-JM-b-CYT-1, ERBB4-JM-b-CYT-2 (Tzahar et al., 1996).

The splice variant JM-a, has an extracellular cleavage site in the stalk-region next to the transmembrane region. The cleavage site can be processed by TACE (tumour necrosis factor- $\alpha$ converting enzyme) and $\gamma$-secretase (Rio et al., 2000; Ni et al., 2001). The processing cleaves the receptor into two fragments: an extracellular and an intracellular one being able to shuttle to the nucleus and acting as transcriptional activator (Williams et al., 2004; Vidal et al., 2005).

The second splice variant defined by the inclusion (Cyt1) or the exclusion (Cyt2) of exon 26. Importantly, exon 26 harbours the binding site for the regulatory domain PIK3R1, promoting PI3K signalling (Elenius et al., 1999; Carpenter, 2003).

The activation of receptor pairs is ligand-dependend and specific. NRG1 and NRG2 bind to ERBB3 and ERBB4, whereas NRG3 and NRG4 seem to bind only to ERBB4. EGF and TGF $\alpha$ activate EGFR only (Marmor et al., 2004, Yarden and Sliwkowski, 2001).

The expression patterns of the receptors in the brain are cell type-dependent. The receptor pair ERBB2/ERBB3 is expressed in glial cells, and the pair ERBB2-ERBB4 in neurons (Buonanno and Fischbach, 2001). ERBB4 is expressed in Parvalbumin positive interneurons (Neddens and Buonanno, 2011). 

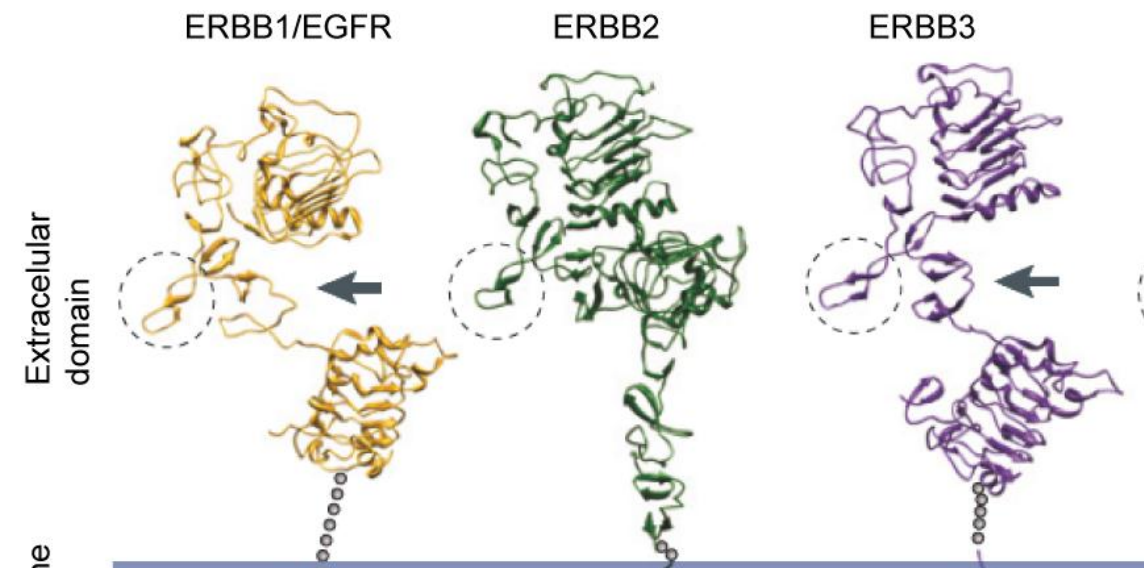

ERBB4
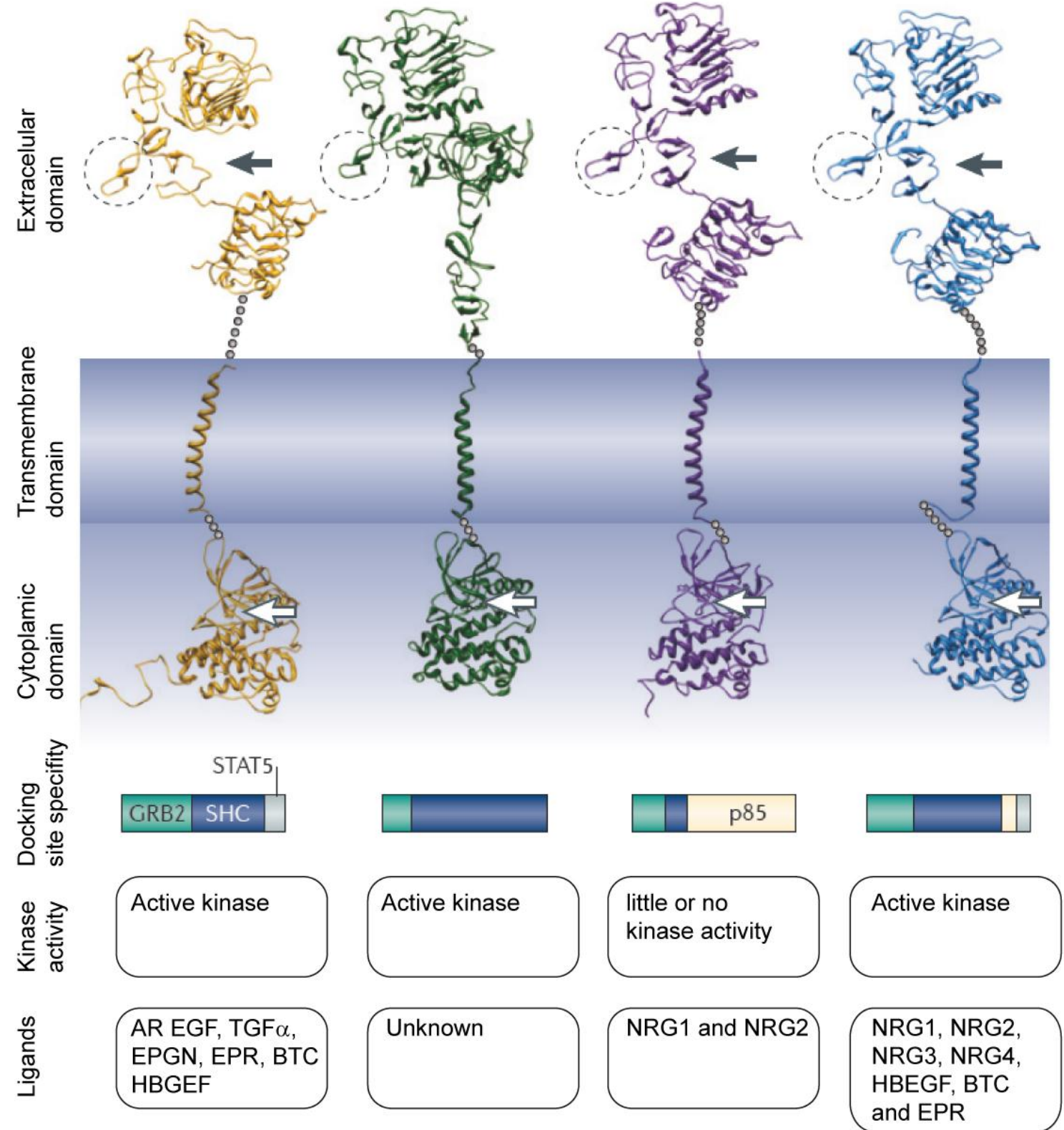

Figure 7: Functional and structural features of ERBB receptors that are unique to each receptor

The black arrows indicate the ligand-binding clefts. The dashed circles indicate the dimerisation loops. The white arrows marks the ATP binding cleft. The sizes of the coloured rectangles below the protein structures represents the fractions of docking site specifity meaning the number of binding sites for each indicated protein normalised to the total number of phosphotyrosine docking sites of each receptors. AR, amphiregulin; BTC, bcellulin; EGF, epidermal growth factor; EGFR, EGF receptor; EPGN, epigen: EPR epiregulin; GBM, glioblastoma; GI, gastrointestinal tract; GRB2, growth factor receptor-bound protein 2; HBEGF, heparin-binding EGF-like growth factor; NRG, neuregulin; NSCLC, non-small-cell lung cancer; SHC, SRC-homology domain-containing; STAT5, signal transducer and activator of transcription 5; TGFa, transforming growth factor-a. Adapted from (Yarden and Pines, 2012). 


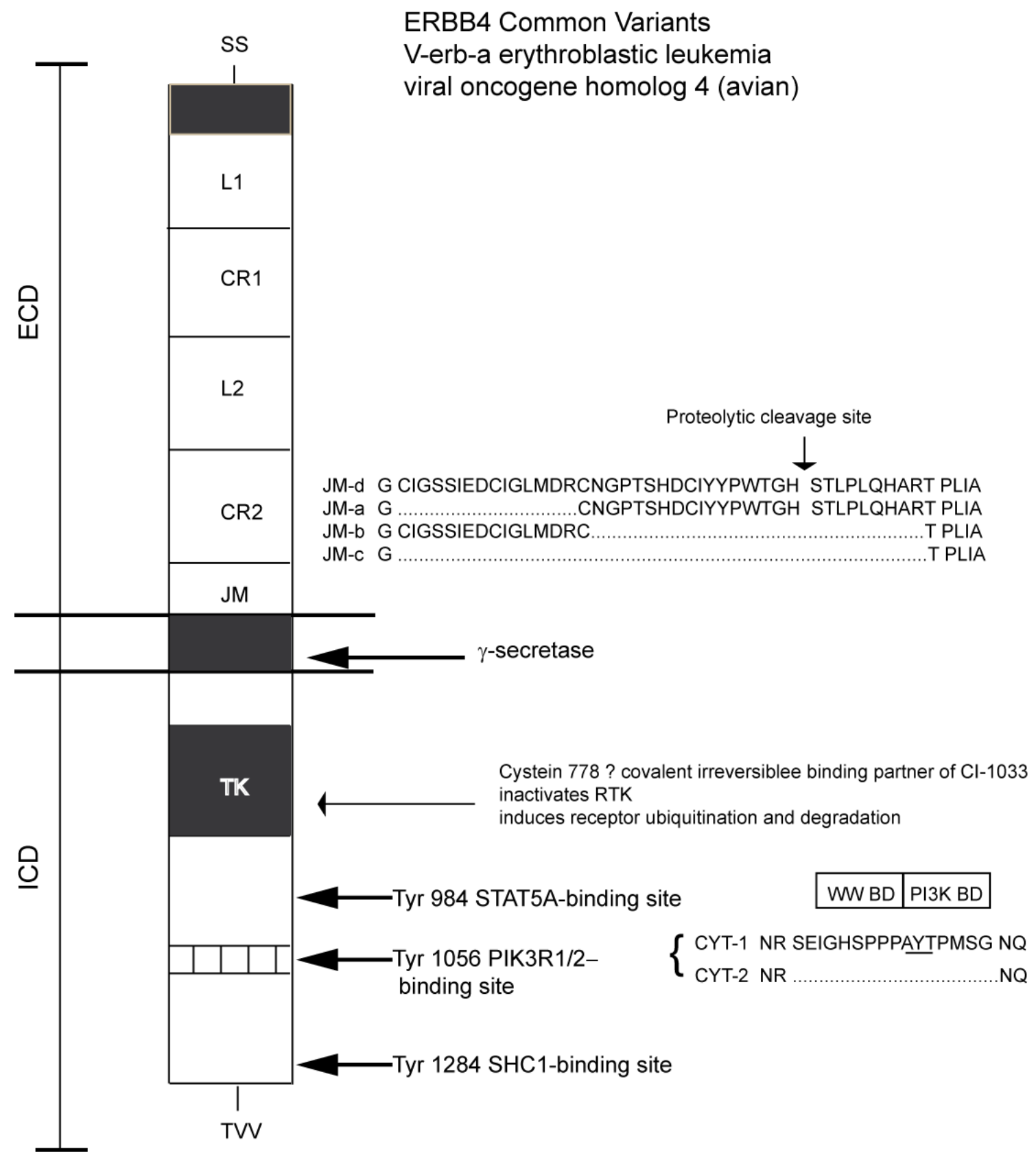

Figure 8: Schematic illustration of ERBB4 domain structure, its variants and binding sites for adapter molecules.

$E C D$, extra cellular ligand binding domain; ICD, intra cellular domain; SS, signal sequence; TK, tyrosine kinase domain; L1,L2, ligand interacting domains; CR1, CR2, cysteine-rich domains; modified from (Zeng et al., 2007b) 


\subsubsection{NRG1-ERBB4 signalling in the nervous system}

The three best characterised signalling pathways initiated through ERBB receptors are the Ras-Mitogen Activated Protein Kinase Ras-MAPK pathway, the PI3K-Akt/PBK pathway and the Phospholipase C/Protein Kinase $\mathrm{C}$ pathway. The STATs (Signal Transducer and Activator of Transcription) can be activated via direct binding to ERBB receptor pairs (Jones et al., 2006; Marmor et al., 2004; Schulze et al., 2005).

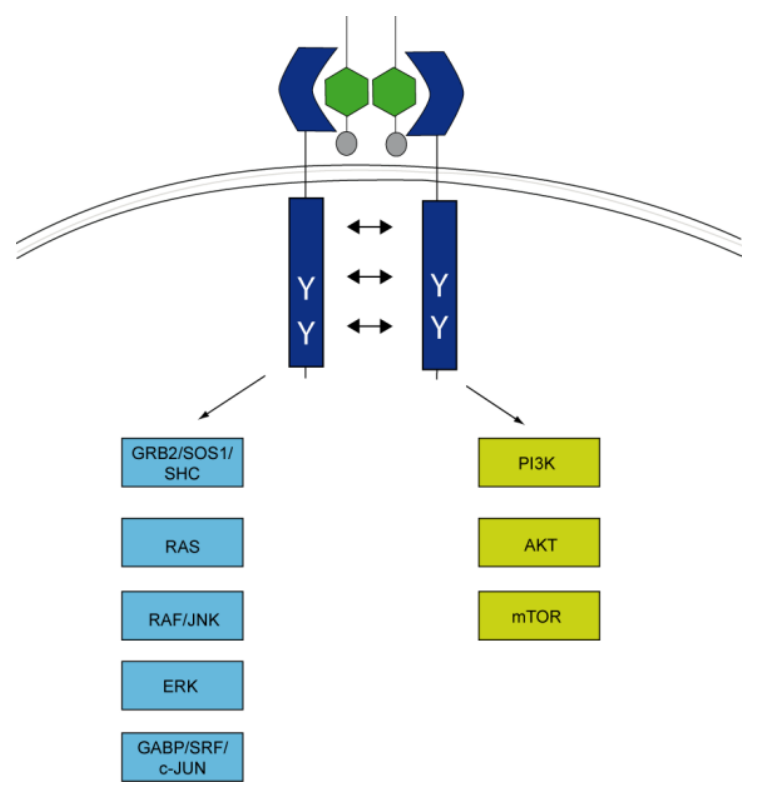

Figure 9: Schematic representation of signalling pathways initialised by ERBB4 receptors.

Modified from: Schizophrenia: Emerging targets and Therapeutic Strategieies (Karam et al., 2010).

\subsubsection{Adapters of ERBB receptors}

In this study 5 different phosph-adapters of ERBB-receptors system have been used.

- PIK3R1 (PI3Kp85 $\alpha$ )

- SRC

- SHC

- STAT5A

- GRB2 


\subsubsection{PI3K}

The phosphoinositide 3-kinase (PI3K) is a nodal point of different pathways in the cell. Its function involves cell growth, proliferation, differentiation, motility, survival and intracellular trafficking. The PI3Ks were first described by Lewis Cantley (Auger et al., 1989). A very recent study confirmed explicitly the role of ERBB4 promoted PI3K signalling in SZ (Law et al., 2012a). PI3Ks are involved in learning and memory and in long-term potentiation (Man et al., 2003). They bind to phospho-tyrosine 1056 of the ERBB4-JMA-CYT1 variant. The recruitment of PI3K to the receptors is promoted by NRG1 i.e. the EGF-like domain (Williams et al., 2009). The PI3 kinases are the central elements of the PI3K/Akt signalling pathway.

The PI3K are subdivided in 3 classes named class I, II, III (Leevers et al., 1999) (Tab. 5).

Class I PI3Ks are activated via G-protein coupled receptors (GPCRs) and receptor tyrosine kinases (RTKs). They form heterodimers and phosphorylate Phosphatidylinositol 3-phosphate (PI(3)P), Phosphatidylinositol (3,4)bisphosphatePI(3,4)P2) and Phosphatidylinositol $(3,4,5)$ trisphosphate ((PI3,4,5)P3).

The Class I PI3Ks consists of two subunits: catalytical subunit p110 with three variants (p110a, p110b and $\mathrm{p} 110 \mathrm{c}$ ) and the regulatory subunit $\mathrm{p} 85$. The regulatory subunit has five variants: the highest expressed are p85a and p55a, followed by p50a, p85b, p55y.

\begin{tabular}{|c|c|c|c|}
\hline group & gene & protein & aliases \\
\hline \multirow{4}{*}{$\begin{array}{l}\text { class I } \\
\text { catalytic }\end{array}$} & PIK3CA & PI3K, catalytic, alpha polypeptide & $\mathrm{p} 110-\mathrm{a}$ \\
\hline & PIKЗCB & PI3K, catalytic, beta polypeptide & p110-b \\
\hline & PIK3CG & PI3K, catalytic, gamma polypeptide & p110-g \\
\hline & PIK3CD & PI3K, catalytic, delta polypeptide & p110-d \\
\hline \multirow{6}{*}{$\begin{array}{l}\text { class I } \\
\text { regulatory }\end{array}$} & PIK3R1 & PI3K, regulatory subunit 1 (alpha) & p85-a \\
\hline & PIK3R2 & PI3K, regulatory subunit 2 (beta) & p85-b \\
\hline & PIK3R3 & PI3K, regulatory subunit 3 (gamma) & p85-g \\
\hline & PIK3R4 & PI3K, regulatory subunit 4 & p150 \\
\hline & PIK3R5 & PI3K, regulatory subunit 5 & p101 \\
\hline & PIK3R6 & PI3K, regulatory subunit 6 & p87 \\
\hline \multirow[t]{3}{*}{ class II } & PIK3C2A & PI3K, class 2 alpha polypeptide & PI3K-C2a \\
\hline & PIK3C2B & PI3K, class 2 beta polypeptide & $\mathrm{PI3K}-\mathrm{C} 2 \mathrm{~b}$ \\
\hline & PIK3C2G & PI3K, class 2 gamma polypeptide & PI3K-C2g \\
\hline class III & PIK3C23 & PI3K, class 3 & Vps34 \\
\hline
\end{tabular}

Table 5. Different classes and variants of the catalytic and regular subunits of the PI3kinases. 


\subsubsection{STAT5A}

The signal transducer and activator of transcription $5 \alpha$ (STAT5A) is a member of the family of seven structurally and functionally related transcription factors (STAT1, STAT2, STAT3, STAT4, STAT5A, STATB, STAT6). Normally STATS are activated by Janus Kinases (JAKs), respond to cytokines (JAK/STAT pathway) and can be phosphorylated by RTKs e.g. EGFR. After phosphorylation STATs homo- or hetero-dimerise and translocate to the nucleus to act subsequently as transcription factors. STAT signalling cascades are associated with cellular transformation (Kisseleva et al., 2002). Disturbed phosphorylation of STATs was found in ErbB4 homozygous KO mice (Tidcombe et al., 2003). STAT5A binds to pY984 ERBB4-JM-a-CYT1 and its increased binding to mErbB4 after Nrg1 stimulation was shown (Wehr, unpublished).

\subsubsection{SHC1}

The $\mathrm{SH} 2$ domains containing transforming protein 1 (SHC1) is the $46 \mathrm{kDa}$ variant expressed by the $S h c A$ mRNA. It is an ubiquitously expressed adapter protein that signals through the mitogen activated protein kinases (MAPK) pathway and takes part in the regulation of apoptosis and drug resistance. The SHC1 protein contains a SH2 domain, a PTB domain and a central region bearing various phosphorylation sites. Null mutant mice homozygous for Shc dye at E11.5 because of heart defects (Ravichandran, 2001; Pelicci et al., 1992). SHC1 binds to phosphorylated Tyr1284 of the ERBB4-JM-a-CYT1 and its increased binding to ErbB4 after Nrg1 stimulation was showen (Wehr, unpublished).

\subsubsection{GRB2}

The growth factor receptor bound protein 2 (GRB2) is an phospo-adaptor protein. It is involved in signal transduction, transformation, cell communication and proliferation. The protein contains one $\mathrm{SH} 2$ two $\mathrm{SH} 3$ domains. It links the EGFR RTKs to the activation of Ras and the MAPKs ERK1 and 2 (Schulze et al., 2005).

\subsubsection{SRC}

The proto-oncogene SRC is a tyrosine kinase. C-Src phosphorylates SHC and STAT5a. For the discovery of this kinase as the first oncogene kinase $\mathrm{J}$. Michael Bishop and Harold E. Varmus were awarded with the Nobel Prize in 1989 (Olayioye et al., 1999; Keely et al., 2000; Sato et al., 2000). 


\subsection{Downstream pathways}

\subsubsection{PI3K/ AKT pathway}

The phosphatidylinositide 3-kinase (PI3K)-AKT-mammalian target of Rapamycin (mTOR) pathway is a key regulator for proliferation, growth, survival, protein synthesis, and glucose metabolism.

PI3Ks convert PIP2 to PIP3. This activates AKT (acute transforming retrovirus (AKT-8) also known as protein kinase B or RAC-PK) a central serine/threonine kinase. AKT is upstream to both mTOR and of GSK3 $\beta$ (Yap et al., 2008) (Fig. 10).

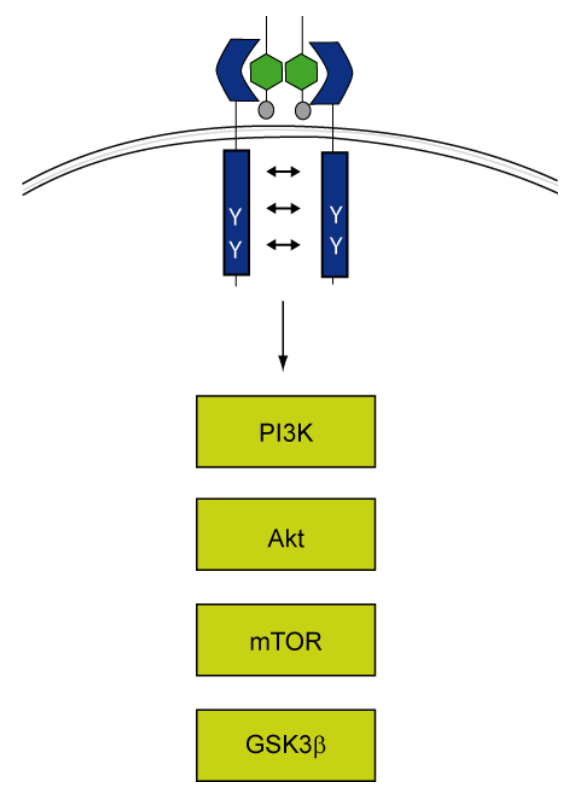

Figure 10: The PI3K, AKT, mTOR pathway

\subsubsection{MAPK /ERK pathway}

The mitogen-activated protein kinase (MAPK) pathway (Fig. 11) is an important central pathway linked to growth, proliferation, differentiation, and survival of various cell types. In general the "MAPK pathway" is a cassette of three kinases, which are sequentially activated. Starting point of the cascade might be e.g. a growth factor.

The very downstream kinase MAPK is activated via phosphorylation through MAPKK (MAPK kinase), which is activated through MAPKKK (MAPKK kinase). The MAPKs are deactivated via MAPK phosphatases (MKPs). In eukaryotic cells at least twelve different MAPKKKs, seven MAPKKs and eight MAPKs active in at least four different modules are described. For example one module 
is the RAF/MEK/ERK cassette. The most upstream kinase is activated by membrane recruitment, usually mediated by adapter molecules, such as Grb2 Sos1 and Shc1. These are recruited to the membrane by an activated RTK. MAPKK is named MEK and the MAPK "extracellular signal-regulated kinase" is called ERK for historical reasons.
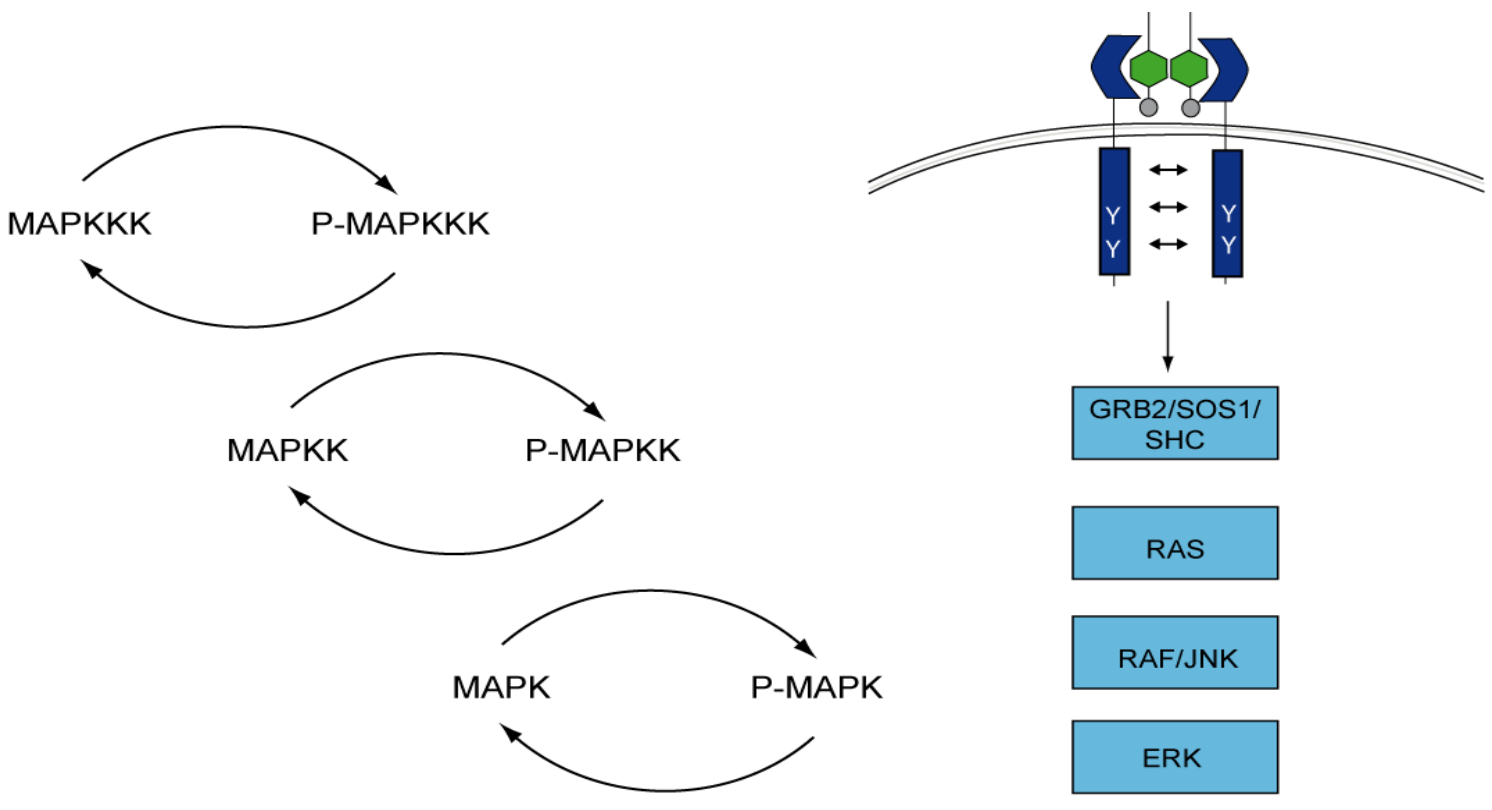

RAF/JNK

ERK

Figure 11 MAPK pathway 


\subsection{Localisation of NRG1-ERBB4 signalling in the neuronal architecture}

\subsubsection{Glutamate hypothesis of SZ}

Glutamate is the most common neurotransmitter in the mammalian brain. Glutamate receptors have excitatory properties and are important for neural development, neuronal survival and synaptic plasticity.

The glutamate receptors in the mammalian CNS are subdivided in:

\begin{tabular}{|l|l|l|}
\hline lonotropic recptors & NMDA & ligand gated ion channels \\
\hline & AMPA & \\
\hline & Kainate & \\
\hline Metabotropic & mGluRs & G-protein coupled \\
\hline
\end{tabular}

Table 6. Overview of the different Glutamate receptors

\subsubsection{NMDA}

Increasing evidence suggests that NMDA hypo-function is involved in the pathophysiology of SZ (Homayoun and Moghaddam, 2007). Low doses of NMDA antagonists like PCP, MK801 or Ketamine can induce SZ-like psychotic symptoms (Bian et al., 2009). PCP and Ketamine lead to decrease parvalbumin (PV) stained interneurons. Reduction of parvalbumin or somatostatin stained interneurons and decrease of mRNA expression of somatostatin, parvalbumin and glutamate acid decarboxylase (GAD 67) are key findings for SZ (Konradi et al., 2011). PCP and Ketamine lead to increased cortical glutamate level. Elevated levels of glutamate are observed in some brain regions of SZ patients.

"At the Network level, psychomimetic doses of NMDA antagonist may favour the balance of excitation over inhibition by blocking NMDA-dependent excitatory inputs to GABAergic interneurons" (Christoph Ott, 2012 personal communication).

"Subcortical NMDA receptor dysfunction, leading to elevated cortical glutamate levels and potentially to excitotoxic processes, may be important, particularly in the early stages of the disease" (Christoph Ott, 2012 personal communication).

mGLu2/3

Recent findings suggest that the compound LY404039 and its oral prodrug LY2140023 targets the metabotropic glutamate system. The effective treatment of SZ patients is still under debate (Mezler et al., 2010). 


\subsubsection{Hyper- and hypo-frontality in SZ}

The gamma frequency oscillations of SZ patients performing working-memory tasks are disturbed. Responsible for this is most likely loss of inhibition of parvalbumin positive $(\mathrm{PV}+)$ interneurons (Uhlhaas and Singer, 2010; Haenschel et al., 2009; Barr et al., 2010).
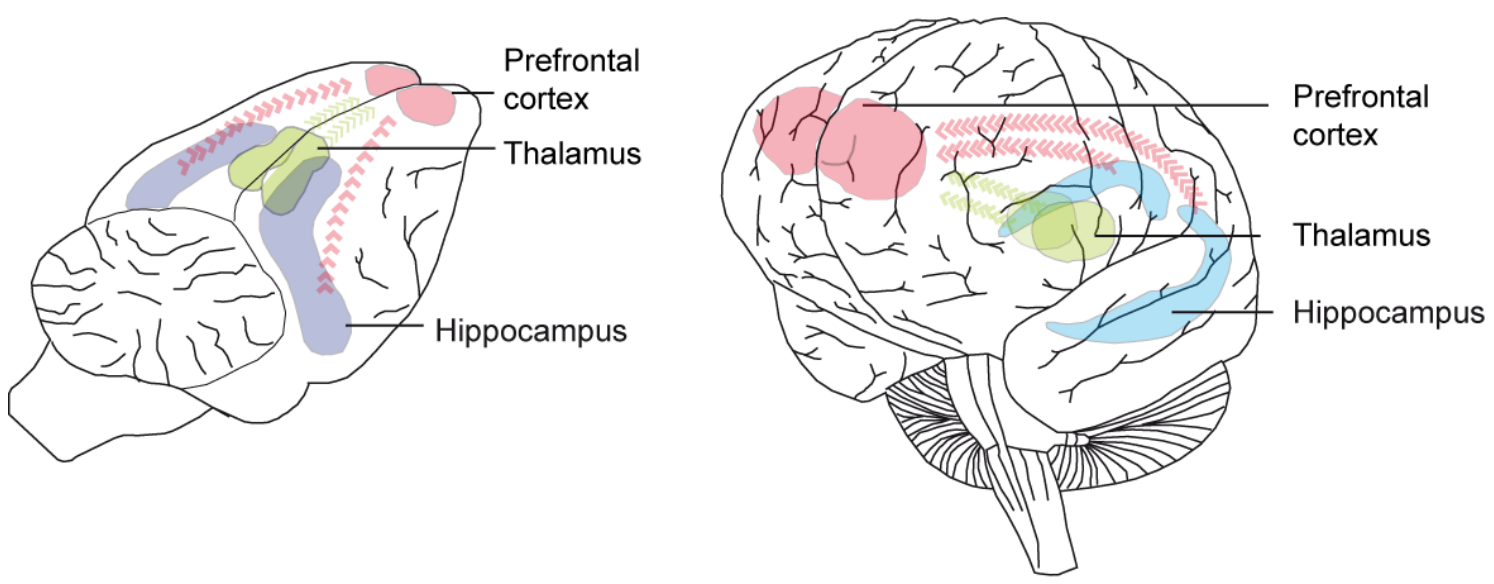

Figure 12: Prefrontal cortex, Thalamus and Hippocampus, connections in mice and man, modified by (Pratt et al., 2012).

Dysfunctional connectivity in schizophrenia. Aberrant activity in both rodent models of the disease (left panel) and in patients (right panel) is centred on prefrontal - hippocampal thalamic networks.

\subsubsection{From neuronal circuits to single synapses}

A small functional substructure of the brain is a simple neuronal circuit. A neuronal circuit is defined as a hierarchical network of inhibitory and excitatory neurons. In the cerebral cortex excitatory glutamatergic pyramidal cells and inhibitory GABAergic neurons form a micro-network (Fig. 13). The pyramidal cells are specialised in the transfer of information between cortical areas and to other parts of the brain. The interneurons function as breaks and oscillation fine tuners to the pyramidal cells. Disturbance of the balance lead to circuit missfunction. In SZ a certain class of interneurons seem to be affected. The PV+ interneurons, including Basket cells and Chandelier cells, localised in the dorsolateral prefrontal cortex show a decrease in Glutamate decarboxylase isoform $67 \mathrm{kDa}$ (GAD67) expression in SZ (Akbarian et al., 1995; Hashimoto et al., 2003; Marín, 2012). Expression of ERBB4 protein in humans and monkeys is restricted to the somatodendritic compartments of interneurons and is absent from pyramidal cells (Neddens et al., 2011; Neddens and Buonanno, 2011; Buonanno, 2010). The precise localisation of ERBB4 in CNS has not been 
described systematically yet and has to be further analysed. For further information see: (Neddens and Buonanno, 2011).

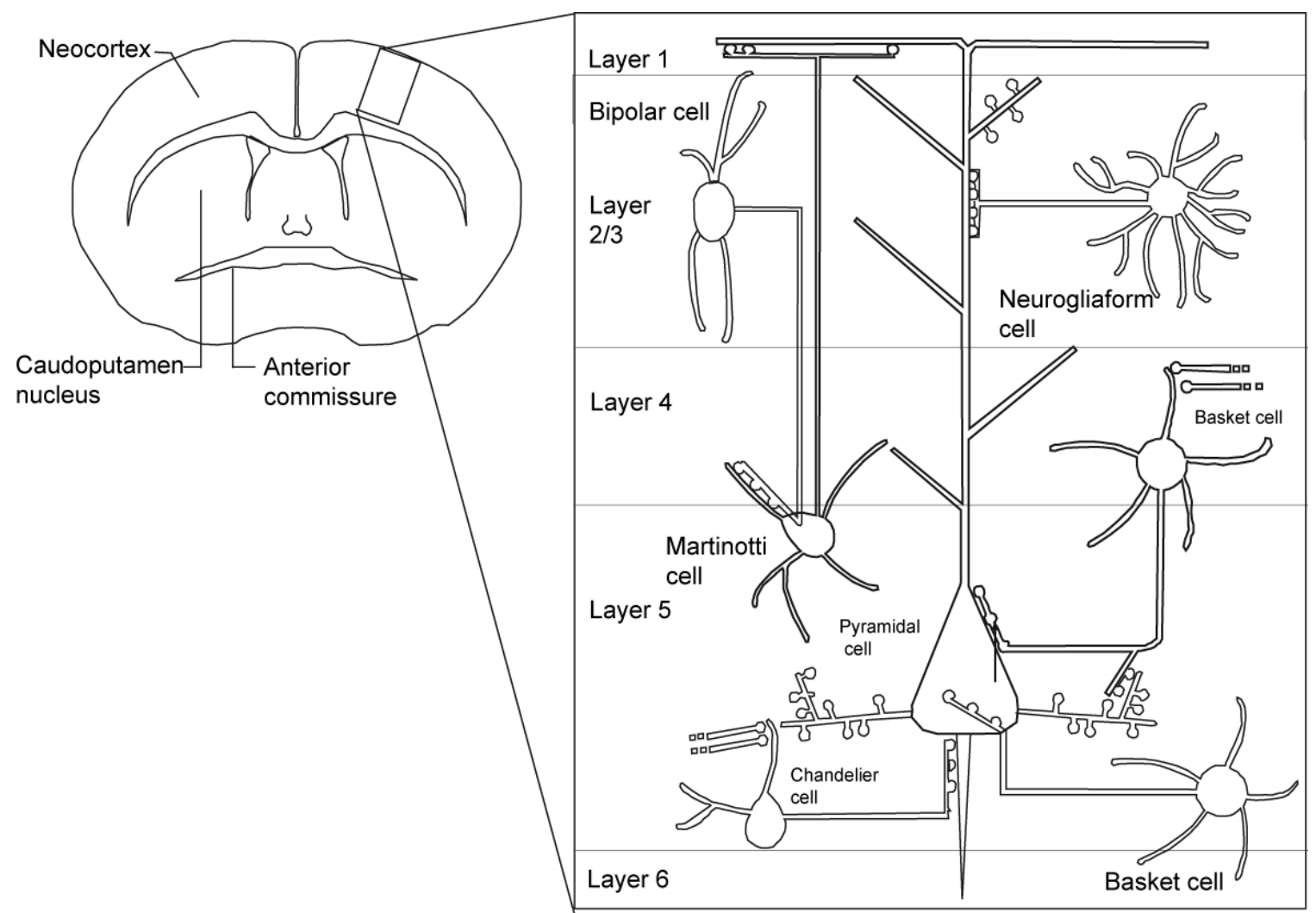

Figure 13: Cortical interneuron network

Different classes of cortical interneurons are distinguished by morphology, neurochemical content, intrinsic electrophysiological properties and pattern of connectivity. Interneurons i.e. Basket cells target the soma and basal dendrites of pyramidal cells, Chandelier cells contact the axon initial segment and Martinotti cells and neurogliaform cells primarily contact the dendrites of pyramidal cells. The cells form a complex network of inhibition and excitation synchronised to a distinct oscillation. Modified from (Marín, 2012).

\subsubsection{Alterations found in the neuronal circuit}

Two different reasons are hypothesised to be responsible for the reduced activity of the PV+ interneurons in SZ.

A loss of function of interneurons might be caused by (I) a reduction of inhibitory synapses number (Woo et al., 1998), or (II) by a failure in the recruitment of the interneurons to their correct final localisation, thereby preventing functional excitation of these inhibitory interneurons (Grunze et al., 1996; Gunduz-Bruce, 2009).

NMDA receptor antagonists or the conditional deletion of NR1 subunit of NMDAR lead to the dis-inhibition of excitatory pyramidal cells and a loss of synchrony, showing SZ like symptoms (Belforte et al., 2010; Carlén et al., 2012). 


\subsubsection{NRG1-ERBB4 in the development of PV+ interneurons}

ERBB4 directs the migration of PV+ interneurons in response to NRG1 (Flames et al., 2004). NRG1-ERBB4 signalling controls the integration and synapse formation of $\mathrm{PV}+$ interneurons with pyramidal cells in neuronal circuits (Fazzari et al., 2010). Moreover, PV+ interneurons deficient in ERBB4, receive less input by pyramidal cells than normal ones (Ting et al., 2011). Interestingly, mice lacking ERBB4 specific in PV+ Interneurons have an impaired working memory phenotype (Wen et al., 2010) resembling cognitive deficits observed in SZ.

An overview of the location and implication of the findings is given in (Fig. 14) for neuronal circuits and in (Fig. 15) for synapse.

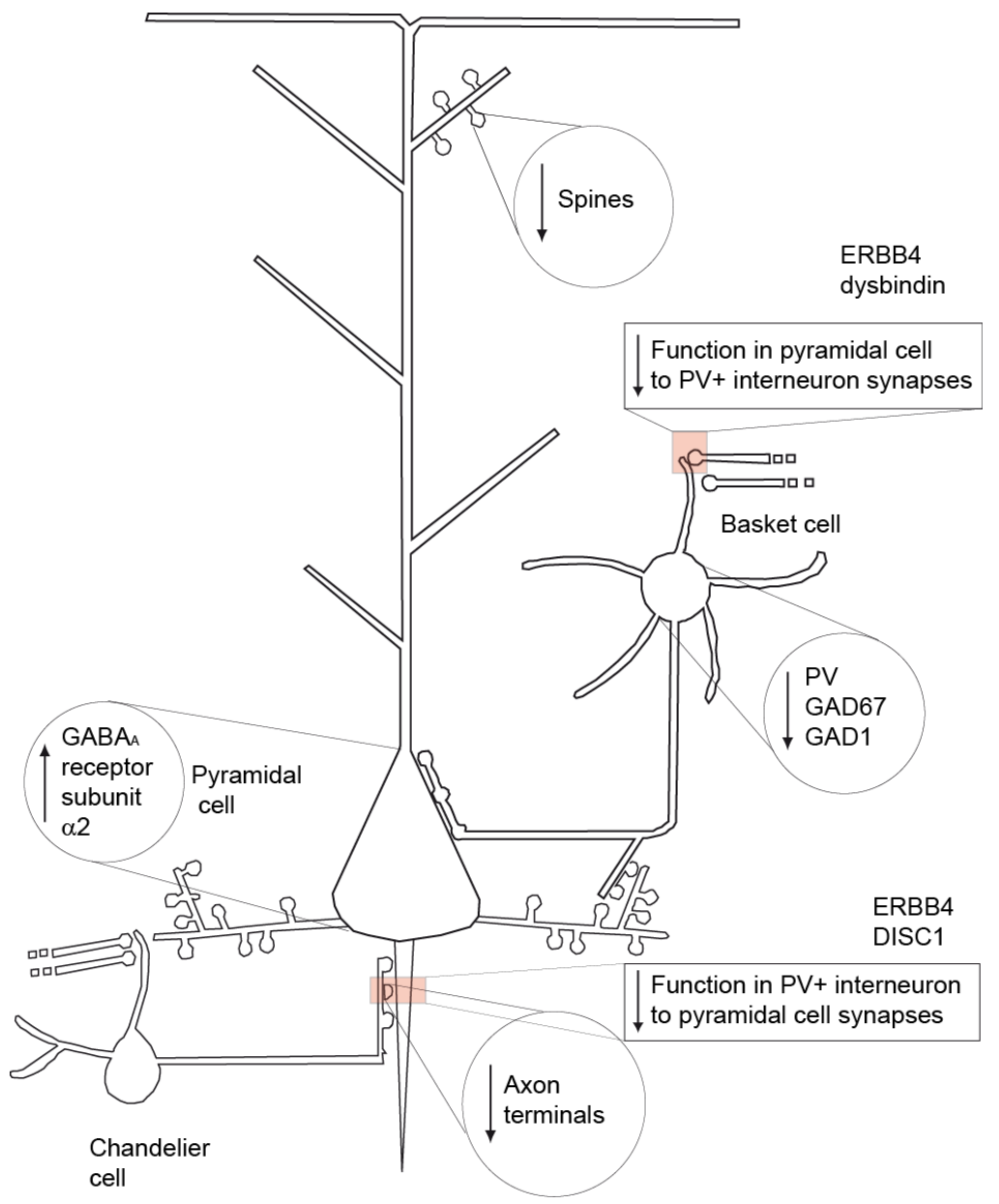

Figure 14: Alterations found in cortical circuits SZ patients and animal models

Spines and $67 \mathrm{kDa}$ glutamate decarboxylase (Gad67) are reduced. Loss of ERBB4 alters presynaptic functions of the Chandelier cells and post synaptic the functions of the Basket cells, resulting in a loss of inhibitory function and synchrony. The Pyramidal cell responds with asynchronous hyperactivity. 


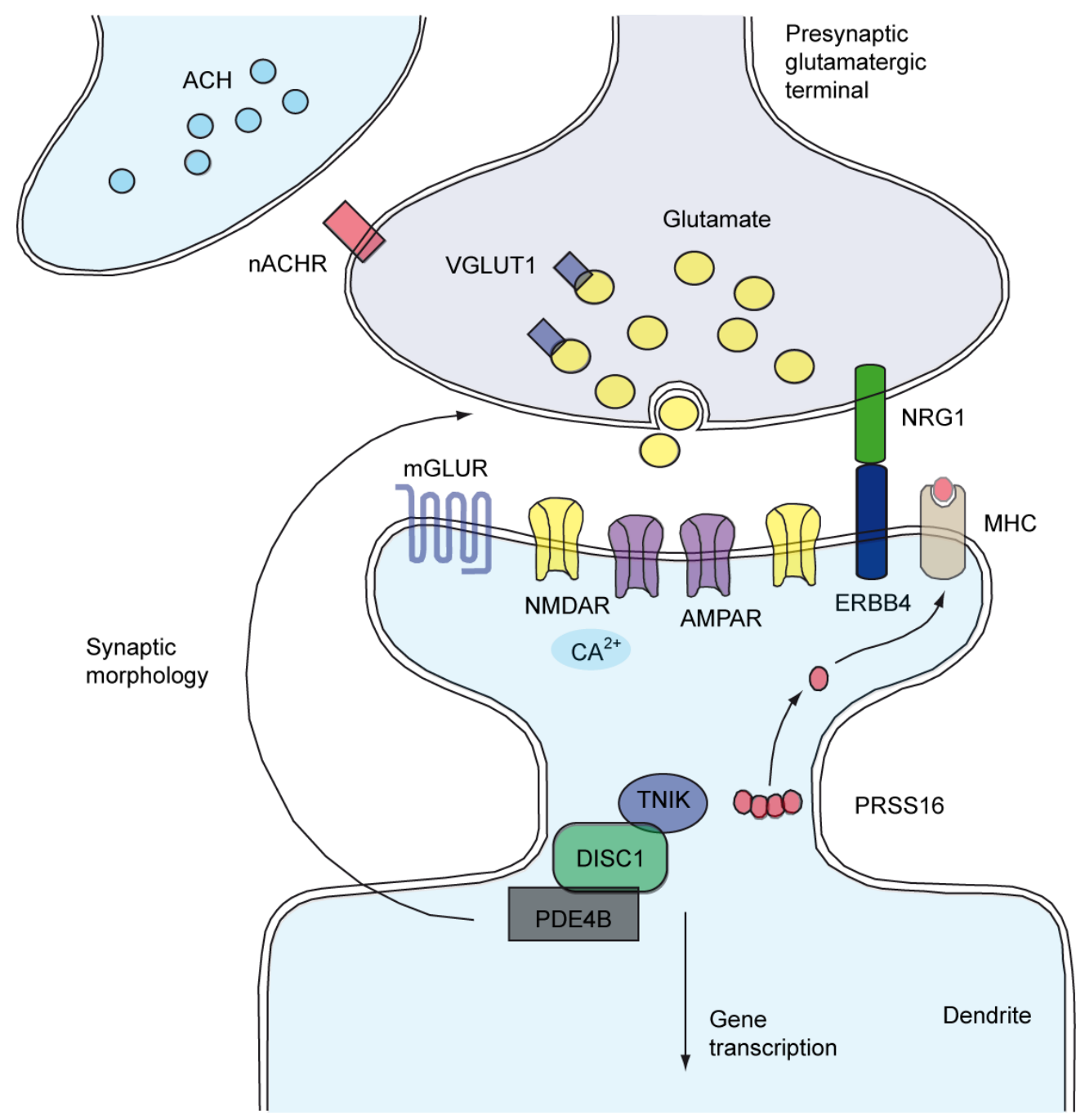

Figure 15 Synapse, modified (Pratt et al., 2012)

The picture shows the most prominent genes implicated in the aetiology of schizophrenia. NRG1-ERBB4 signalling regulates the function and morphology of the synapse. DISC1 coordinates signalling pathways that regulate synaptic structure (DISC1 with phosphodiesterase 4B (PDE4B) and TRAF2 and NCK-interacting kinase (TNIK)) a7 subunit containing nicotinic acetylcholine receptors (nAChRs) are strongly implicated in disease risk via gene copy number variations. Major histocompatibility complex (MHC) molecules have some role in the maintenance and plasticity of synaptic connections. AMPA: (a-amino3hydroxy-5methyl-4-isoxazole propionic acid receptor (AMPAR); mGluR: metabotropic glutamate receptor; NMDAR: N-methyl-D-aspartate receptor; PRSS16: serine protease 16; VGLUT1: vesicular glutamate transporter 1. 


\subsection{Targeting NRG1-ERBB4 with protein-protein interaction assays}

\subsubsection{First generation drugs}

The first class of drugs also named 'typical' drugs used in the treatment of SZ is termed neuroleptics and act as antipsychotic drugs. Neuroleptics were found in MDMA (3,4-methylenedioxy-N-methylamphetamine) and PCP (Phencyclidine) induced psychotic mice models. All of these drugs target the dopamine system. They bind to the dopamine D2 receptor subtype and block them in the mesolimbic dopaminergic system.

Prominent first generation drugs are Chlorpromazine (first synthetised in 1950) and Haloperidol (1958).

These drugs display severe side effects. The dopamine blockade in the nigostratial and hypothalamic-pituitary systems lead to extrapyramidal side effects mimicking Parkinson's disease, hyperprolactinaemia, sedation metabolic-syndrome and hypotension. The patients have to take the drugs for their whole life. Moreover, the first generation drugs are used only for symptomatic treatment and they do not affect the cause of the disease (Hudepohl and Nasrallah, 2012).

\subsubsection{Second generation drugs}

Second generation drugs, also named 'atypical' drugs, are multi-affinity receptor target agents targeting specific D2 receptor subtypes or compounds with a balanced activity against serotonin (5-hydroxytryptamine) versus dopamine.

Atypical drugs, even if failing to treat all symptoms of SZ, treat a broader set of symptoms than first generation drugs and display a diminished side effect profile due to reduced extrapyramidal side effects. They also target specifically dopamine receptors in various degrees to perform the therapeutic action. Prominent representatives of atypical drugs are Clozapine (Barros 2011), which reverses altered spine formation in NRG ERBB2-4 mice, Olanzapine and Risperidone (Penzes et al., 2011).

Typical and atypical drugs that target dopamine receptors support the dopamine hypothesis of SZ. Nevertheless, they fail in curing SZ and treat only some symptoms with occasionally tremendous side effects. Principally, no accelerated progress in SZ-drug development in the past 50 years has been observed. As the construct validity of SZ in animal models cannot be fully accomplished, the complexity of an animal model obscure smaller effects, costs for animal experiments and maintenance are high and usability of animals in 
HTP screenings are limited, animal models seem not to be the right system for large compound screening.

At least, knowledge of the genetic risk factors of SZ should be tried to be translated into artificial systems, resembling partially valid models to perform HTP screens identifying chemical lead compounds to develop new classes of substances which subsequently need to be active in the corresponding translational/genetic mouse model.

\subsubsection{Classic hit to lead discovery}

Most screens have been performed with in vitro assays. Substance libraries were tested against "naked" proteins. A hit generated in this kind of assay has to be evaluated extensively afterwards and may i.e. often not enter cells or tissues.

Hit confirmation means first retesting of the hits in the same assay system with the same assay conditions. Than, dose-response curves are generated to determine key pharmacological hallmarks (sensitivity, efficacy etc.). Orthogonal testing follows. The hits are tested in different assays or with different technologies. In a secondary screening the hits are tested in a cellular environment to ensure membrane permeability. Chemical amenability /accessibility has also to be ensured. Intellectual property is the next everpresent issue for commercial screens.

Biophysical testing is used to access if the compound binds to the target, i.e. nuclear magnetic resonance or isothermal titration calorimetry.

After hit confirmation, hit expansion follows. Compound clusters from the screen are chosen and expanded. The clusters contain compounds with high target affinity (below 1uM), low molecular weight and moderate lipophilicity. The compounds should not interfere with the P450 enzymes or bind to the human serum albumin. The compounds should also be stable, soluble in water, and should be cell permeable, they should further not exhibit cytotoxicity and should not be rapidly metabolised.

The compounds have to show significant biological activity in cellular assays and selectivity versus other related targets.

Lead optimization is the later process of chemical modification of lead (hit) compounds to get more potent leads with less off-target active structures (Bleicher 2003, Astle2010). 


\subsubsection{Compound screens}

Compound screens give the opportunity to test large numbers of drugs, compounds or lead structures for primary effects on proven therapeutically relevant targets. For subsequent structural modifications (medicinal chemistry), it is important to identify the exact mechanism or mode of action. For HTPcompound screens an assay should be reliable, robust, scalable, fast in response, easy to readout, and mimicking the mechanism observed as close as possible.

Proximal outputs of the NRG1-ERBB4 signalling system can be monitored with PPI assays i.e. at the level of receptor dimerization or the phospho-adapter recruitment. For these types of analyses the following assay-systems should be discussed.

\subsection{Cell based assays}

PPIs can be analysed with genetic or biochemical methods. Genetic methods contain e.g. FRET (Fluorescence Resonance Energy Transfer), BRET Bioluminescence Resonance Energy Transfer) or complementation systems like yeast to hybrid. Biochemical methods include e.g. protein complex immunoprecipitation (Co-IP) (Boute et al., 2002; Uetz and Hughes, 2000). The accurate method depends on the questions asked and the usability e.g. in high throughput or fully automated systems.

\subsubsection{Biochemical methods e.g. Co-IP}

The Co-IP is based on a classical immunoprecipitation. A protein of interest is pulled down from a lysate via a specific antibody. If the protein is interacting with a second protein this protein is bound to the first protein. A second antibody is then used to probe against the second protein and so to detect the PPI. Unfortunately, the interaction can take place in vitro in the lysate which might lead to artefacts in the detection. A quantification of the PPI can be done via ImageJ or other image programs. The method is cost and labour intensive and difficult to automate in bigger scales.

\subsubsection{Affinity columns}

A peptide is bound to an affinity column. If a cell lysate is loaded on the column and proteins interacting with the peptide sequence bind to the peptide and can be purified afterwards. The putative interaction partners can be further analysed via MALDITOF etc. 


\subsection{Protein fragment complementation assays}

\subsubsection{Yeast two hybrid}

The Yeast two-hybrid $(\mathrm{Y} 2 \mathrm{H})$ assay is a protein fragment complementation assay to measure PPI or protein-DNA interactions. In the classical version, the LacZ reporter gene is under the control of the upstream activating sequence UAS. The UAS transcription factor Gal4 is fragmented in an UAS binding domain and a transcriptional activator domain. Both domains can be fused to two putative interacting proteins of interest (pray and bait), and if these proteins come to close proximity, modify the Gal4 transcription factor and activate the reporter gene. The $\mathrm{Y} 2 \mathrm{H}$ is a fast and scalable low-tech assay.

On the other site yeast is not the ideal organism to express mammalian proteins due to lack of specific chaperons and localisation mechanisms: Furthermore, interactions that take place beside the nucleus cannot be detected and this method produces a high false positive failure rate. In conclusion, $\mathrm{Y} 2 \mathrm{H}$ is not suitable to detect interactions of huge mammalian membrane proteins (Stagljar et al., 1998).

\subsection{Split TEV}

The split TEV method is a protein fragment complementation assay, based on the $\mathrm{NiA}$ inclusion protease from the tobacco etch virus (TEV). The protease is split into an $\mathrm{N}$ - and a C-terminal part (NTEV + CTEV), thereby setting apart the catalytic residues. When NTEV and CTEV are fused to proteins of interest, which are capable of interacting with each other, the TEV protease activity is reconstituted as the fragments come into close proximity. In turn, the protease activates an artificial reporter by cleaving at a highly specific site encoded by ENLYFQ'G/S. Artificial reporters can be of transcriptional nature or reporter protein-based(Wehr et al., 2006).

A direct reporter protein is i.e. an inactive luciferase enclosed by two mutated Estrogen receptor domains (ERT2) domains bound via TEV cleavage sites (tevS). The two large ERT2 domains prevent luciferase from proper folding to functional form. TEV protease activity releases the luciferase from the ERT2 domains, it folds to a functional confirmation and the activity of luciferase as a readout can be measured(Wehr et al., 2006).

Transcription based system use i.e. the artificial transcription factor galactose 4viral protein $16(\mathrm{GV})$. The GV is bound with the tevS to one of the to interacting proteins with split TEV protease halves. Reconstitution of TEV-protease activity cleaves the tevS and releases the GV to shuttle to the nucleus and activate a 
reporter protein under the control of the upstream activator site of five GAL4 responsive elements (UAS) site. A reporter protein can be i.e. a fluorescent protein like enhanced green fluorescent protein (EGFP) or an enzyme like the Firefly luciferase. Using a transcription factor and an enzyme as read out allowes also a high magnification of the signal.

The assay is robust, scalable, and was shown to be highly specific for ERBB receptors. It constitutes an optimal assay to access the intercellular NRG1ERBB4 signalling cascade potentially adaptable to a HTP-screen (Wehr et al., 2006; Wehr et al., 2008a; Djannatian et al., 2011).

\subsection{2 z'-Factor}

For HTP screening, the z'-factor is a statistical determinant of the robustness and reliability of the assay. It links a large number of single measurements of unknown effects and effect sizes to a set of positive and negative controls. The $z$ 'factor is calculated for small (or pilot) screens to predict if the assay is qualified for HTS. Four parameter are used, the means $(\mu)$ and the standard deviations $(\sigma)$ of the positive $(p)$ and the negative $(n)$ controls ( $\mu p, \sigma p$ and $\sigma n$, $\mu n)$.

$z^{\prime}$-factor $=1-\frac{3(\sigma p+\sigma n)}{|\mu p-\mu n|}$

\subsubsection{The interpretation of the $\mathrm{z}^{\prime}$-factor:}

- 1 is the ideal $z^{\prime}$-factor. It can never exceed and is impossible to matched.

- 1-0.5 is an excellent assay. 0.5 is the value when $\sigma p=\sigma n, \mu p$ and $\mu n$ show a separation of twelve standard deviations.

- $0.5-0$ is a marginal assay. The assay is not automatically qualified for HTP screening. For complicated but stable assays lower z'-factors of e.g. 0.4 can be defined as limit.

- 0 is a yes/no assay with no separation window, overall not suitable for HTP screening.

- Less than 0, the assay is not qualified for HTP screening, as positive and negative controls do not have any separation window and overlap (Zhang et al., 1999; Zhang et al., 2008; Zhang, 2008; Sui and Wu, 2007). 


\subsection{Aim of the thesis}

$\mathrm{SZ}$ is a severe disease. To date there are no drugs available, which mediate or attenuate the negative symptoms and cognitive deficits. Genetic studies showed that the NRG1-ERBB4 signalling system is altered at least in a subgroup of SZ patients. Moreover, the NRG1-ERBB4 signalling system most likely influences biological processes that are central to the diseases (i.e. interneuron migration and function with an impact on the excitatory-inhibitory balance and associated working memory and cognitive deficits). Therefore, addressing NRG1-ERBB4 signalling is one of the most promising targets for the development of new drugs for SZ.

The first main aim of the thesis is the design, development and characterisation of a split TEV-based co-culture assay to monitor the most proximal steps of the NRG1-ERBB4 signalling cascade. The assay must be sensitive, robust and adaptable to high-throughput approaches for future screenings of huge compound libraries. The second main aim is the engineering and validation of the secondary assays useable to analyse putative hits from larger screens.

As a pilot study, the developed assay will be used to screen 727 FDA approved drugs obtained from the NIH collection. The recovered hits will be further validated by an extensive set of secondary assays containing different Nrgs, ERBB receptors and adapter proteins, to get a fast and clear insights into the specificity of drug function, and to discard false positive hits. The aims of the screening project are (1) the detection of unknown therapeutic potentials in drugs available already on the market, and (2) the identification of new lead structures from tested substances for further lead optimisation processes. 


\title{
3 Chemicals and Reagents
}

Chemicals were received from Amersham Biosciences, BD Falcon, BioRad, Carl Roth, Eppendorf, Invitrogen, Merck, Riedel de Haen, Roche, Serva, Sigma and VWR.

\subsection{Laboratory suplies}

Consumable supplies, which are not mentioned separately were purchased from commercial sources as BD Falcon, Eppendorf, Gilson, Greiner-Nunc and Menzel-Gläser.

Cell culture dishes

BD-Falcon

$15 \mathrm{~cm}$

$10 \mathrm{~cm}$

$5 \mathrm{~cm}$

6 well

12 well

24 well

Cryo tubes

ECL hyperfilms

Electroporation cuvettes $(1,2,4 \mathrm{~mm})$

NuPAGE 4-12\%Bis-Tris Gels

PVDF Membrane Hybond $P$

Reaction tubes

Nunc

Amersham Bioscience

Biorad

Invitrogen

Amersham Biosciences

Eppendorf

\subsection{Laboratory equipment}

\section{Hardware}

Atroum 611 Water Putification System

Sartorius

Gene Pulser Xcell

Biorad

Hood

Heraeus

Microplate reader Mithras LB 940

Berthold Technologies

Microscopes

Leica

Leica DM IRBE (invers)

Leica DM RXA

Leica TCS SP5 X (confocal)

Nucleofector

SDS PAGE gel electrophoresis system

Xcell SureLock NuPAGE system

Sonicator Bandelin Sonoplus

X-ray film developer KODAK XOMAT

CellStarRoboter

\author{
Amaxa \\ Invitrogen \\ Invitrogen \\ Bandelin \\ Kodak \\ Hamilton
}

EDV Hardware

Canon iRC3580

Canon 
HP Color Laserjet 4550

Laptop Lenovo T61

iMac 11,2

\section{EDV Software}

Adobe package

DNASTAR Lasergene

Firefox

Microsoft Office 2003

Microsoft Office $X$

Zotero

Chemsketch

\section{Kits}

Calf intestine phosphatase (CIP)

Nucleobond PC 20

NucleoBond Xtra Midi EF

Nucleobond PC 500 EF

Nucleobond PC 10.000 EF
Hewlet Packard

Apple

Adobe

DNAStar

Mozilla Foundation

Microsoft

Microsoft

www.zotero.org

ACD Labs

Roche

Machery-Nagel

Machery-Nagel

Machery-Nagel

Machery-Nagel

\subsubsection{Antibodies}

\begin{tabular}{|l|l|l|}
\hline Name & Anti & Supplier \\
\hline Phospho-Akt (Ser473) & Rabbit & Cell Signaling Technology \\
\hline Akt & Rabbit & Cell Signaling Technology \\
\hline FLAG M2 & Mouse & Sigma Aldrich \\
\hline HA & Rat & Roche \\
\hline c-Myc & Mouse & Santa Cruz Biotechnology \\
\hline V5 & Mouse & Invitrogen \\
\hline Phospho-p70 S6 Kinase (Thr389) (108D2) & Rabbit & Cell Signaling Technology \\
\hline p70 S6 Kinase & Rabbit & Cell Signaling Technology \\
\hline Phospho-HER4/ErbB4 (Tyr984) & Rabbit & Cell Signaling Technology \\
\hline p-ErbB4 (Tyr 1056) & Rabbit & Santa Cruz Biotechnology \\
\hline $\begin{array}{l}\text { Phospho-p44/42 MAPK (Erk1/2) (Thr202/ Tyr204) } \\
\text { (D13.14.4E) XP }\end{array}$ & Rabbit & Cell Signaling Technology \\
\hline p44/42 MAPK (Erk1/2) (137F5) & Rabbit & Cell Signaling Technology \\
\hline ErbB-4 (C-7) & Mouse & Santa Cruz Biotechnology \\
\hline Phospho-HER4/ErbB4 (Tyr1284) (21A9) & Rabbit & Cell Signaling Technology \\
\hline GSK-3? (27C10) & Rabbit & Cell Signaling Technology \\
\hline Phospho-GSK-3?/? (Ser21/9) & Rabbit & Cell Signaling Technology \\
\hline
\end{tabular}

Table 8. Antibodies used 


\subsubsection{Oligonucleotides}

Were synthezised in house by MPI-EM core facility Primers

Delivery concentration

$50 \mathrm{pmol} / \mathrm{ul}$

f.c. in the PCR

$0,2 \mathrm{uM}$

\subsubsection{Plasmids}

$\begin{array}{ll}\text { Adapters } & \\ \text { HS306 PIK3R1-CTEV-2HA } & \text { KanR } \\ \text { HS309 SRC-CTEV-2HA } & \text { KanR } \\ \text { HS310 GRB2-CTEV-2HA } & \text { KanR } \\ \text { HS311 STAT5A-CTEV-2HA } & \text { KanR } \\ \text { HS316 SHC1v2-CTEV-2HA } & \text { KanR } \\ & \\ \text { RTKs } & \\ \text { HS275 ERBB4-Glink-NTEV-tevS-GV-2HA } & \text { AmpR } \\ \text { HS276 ERBB4-Glink-CTEV-2HA } & \text { KanR } \\ \text { HS322 EGFR-Glink-NTEV-tevS-GV-2HA } & \text { AmpR } \\ \text { HS323 EGFR-Glink-CTEV-2HA } & \text { KanR } \\ \text { HS324 ERBB2-Glink-NTEV-tevS-GV-2HA } & \text { AmpR } \\ \text { HS325 ERBB2-Glink-CTEV-“HA } & \text { KanR } \\ \text { HS326 ERBB3-Glink-NTEV-tevS-GV-2HA } & \text { AmpR } \\ \text { HS327 ERBB3-Glink-CTEV-2HA } & \text { KanR } \\ & \\ \text { Split TEV } & \text { KanR } \\ \text { TC118 TM-TEV } & \text { KanR } \\ \text { TC97 } & \text { TM-tevS-GV } \\ \text { TC84 } \quad \text { pCMV-GV } & \\ & \\ \text { NRGs } \quad \text { Neuregulin1.I-beta1a } & \\ \text { V368 } & \text { Neuregulin1.II-beta1a } \\ \text { V369 } & \text { Neuregulin1.II-beta1a }\end{array}$

Other

TM-FRB_IRES_FKBP

HTR5A-NTEV-tevS-GV-2HA

$\beta$-Arrestin2 $\delta$-CTEV-2HA

\section{Gateway backbone vectors}

pDest-F

pDest-HA

pDest-V5

pDest-Glink-NTEV-tevS-GV-2HA

pDest-Glink-CTEV-2HA

\subsubsection{Bacterial strains}
E. coli $\mathrm{DH} 5 \mathrm{a}$
E. coli DH10B
E. coli XL1-blue
E. coli One shot Mach 1-T1
E. coli DB3.1 ccdb survival

Invitrogen

Invitrogen

Statagene

Invitrogen 


\subsubsection{Mammalian cell lines}

HEK293

HEK293FT

PC12

PC12TO

PC12

Neuroscreen-1

Oli-neu

NIH3T3

SHSYY5
Human Embryonic Kidney immortalised cell line

(ATTC) (DuBridge et al., 1987)

Human Embryonic Kidney immortalised cell line

(ATTC) (Javanbakht et al. , 2003)

Rat adrenal pheochromocytoma cell line

(ATTC) (Grene and Tischler. 1976)

expresses TetR-VP16 transactivator (Clontech)

optimised for screening PC12 cells (Thermo Fisher Scientific.

Murine oligodendroglial precursor immortalised cell line

(Jung et al.,1995)

mouse embryonic fibroblasts

(Todaro and Green, 1962)

Neuroblastoma cells)

\subsubsection{Solutions and buffers}

PBS (Phosphate buffered saline) 10x

$\mathrm{NaCL} \quad 100 \mathrm{~g}$

$\mathrm{KCl} 2,5 \mathrm{~g}$

$\mathrm{NA} 2 \mathrm{HPO} 4 \times 2 \mathrm{H} 2 \mathrm{O} \quad 7,2 \mathrm{~g}$

$\mathrm{KH} 2 \mathrm{PO} 4 \quad 2,5 \mathrm{~g}$

Disolve, adjust $\mathrm{pH}$ to 7,2 with $\mathrm{NaOH}$, add $\mathrm{H} 2 \mathrm{O}$ to $1000 \mathrm{ml}$

TBS (TRis buffered saline), $\quad$ 10x

Tris-Base (1M; pH 8) $\quad 50 \mathrm{ml} \quad$ (f.c. $50 \mathrm{mM}$ )

$\mathrm{NaCl}(5 \mathrm{M}) \quad 30 \mathrm{ml} \quad$ (f.c. $150 \mathrm{mM}$ )

\subsubsection{Buffers for molecular biology}

DNA sample buffer, 10x

$\begin{array}{ll}\text { Bromphenolblue } & 0,25 \% \\ \text { Xylencyanol } & 0,25 \% \\ \text { Ficoll (Type400) } & 15 \%\end{array}$

In ddH2O

TAE (Tris /Acetat/EDTA) buffer, 50x

Tris-Base $\mathrm{pH} 8$

$\operatorname{EDTA}(0,5 \mathrm{M} ; \mathrm{pH} 8)$

Add ddH2O to $1000 \mathrm{ml}$

TE (Tris - EDTA) buffer,

Tris-Base $\mathrm{pH} 7,4$

$\operatorname{EDTA}(0,5 \mathrm{M} ; \mathrm{pH} 8)$

Ethidiumbromid

$\mathrm{EtBr} 1 \%$ in ddH2O

Final concentration in the gel

dNTP mix 50x

dATP, dCATP, dGTP, dTTP $\quad 10 \mathrm{mM}(2,5 \mathrm{mM}$ each $)$

f.c. in the PCR
$10 \mathrm{mM}$

$1 \mathrm{mM}$

$242 \mathrm{~g}(2 \mathrm{M})$

$100 \mathrm{ml}(1 \mathrm{mM})$

$10 \mathrm{mg} / \mathrm{ml}$

$1 \mathrm{ug} / \mathrm{ml}$

200uM (50uM each)

LB-medium (Luria and Bertani medium / Luria brooth) (BERTANI, 1951)

Bacto Yeast extract

$0,5 \%$ 
Bacto Peptone

$\mathrm{NaCl}$

Optional Agar

SOC - medium

Bacto Yeast extract

Bacto Peptone

$\mathrm{NaCl}$

Glucose

$\mathrm{KCl}$

$\mathrm{MgSO} 4$

Antibiotics

Kanamycin

Ampicilin

Chloramphenicol

Gentamycin

Zeocyn ( low salt agar)
$1 \%$

$1 \%$

$1 \%$ for plates

$0,5 \%$

$2 \%$

$10 \mathrm{mM}$

$20 \mathrm{mM}$

$2,5 \mathrm{mM}$

$10 \mathrm{mM}$

f.c. in agar plates

$50 \mathrm{ug} / \mathrm{ml}$

$100 \mathrm{ug} / \mathrm{ml}$

\subsubsection{Luciferase assay buffers}

Lysis-buffer

Passive lysis buffer $\quad(5 x) \quad$ Promega (Cat\# E1941)

Dilute $30 \mathrm{ml}$ of $5 x$ Buffer in $120 \mathrm{ml} \mathrm{ddH}_{2} \mathrm{O}$

Firefly-luciferase-assay buffer (Gaunitz and Papke, 1998)

$\begin{array}{llll}\text { Tricine } & & 500 \mathrm{ml} & 1500 \mathrm{ml} \\ \left(\mathrm{MgCO}_{3}\right)_{4}{ }^{*} \mathrm{Mg}(\mathrm{OH})_{2}{ }^{*} \mathrm{HH}_{2} \mathrm{O} & 20 \mathrm{mM} & 1792 \mathrm{mg} & 5376 \mathrm{mg} \\ \left(\text { Magnesiumcarbonat-Hydroxid-Pentahydrat) }^{1,07 \mathrm{mM}}\right. & 260 \mathrm{mg} & 780 \mathrm{mg} \\ \mathrm{MgSO}_{4} & 2,67 \mathrm{mM} & 161 \mathrm{mg} & \\ \text { EDTA } & 0,1 \mathrm{mM} & 100 \mu \mathrm{l}(0,5 \mathrm{M}) & 483 \mathrm{mg} \\ \text { DTT } & 33,3 \mathrm{mM} & 2570 \mathrm{mg} & 700 \mu \mathrm{l} \\ \text { Coenzym A } & 270 \mu \mathrm{M} 1 & 05 \mathrm{mg} & 315 \mathrm{mg} \\ \text { D-Luciferin, free base } & 470 \mu \mathrm{M} & 66 \mathrm{mg} & 198 \mathrm{mg} \\ \text { ATP } & 530 \mu \mathrm{M} & 146 \mathrm{mg} & 438 \mathrm{mg}\end{array}$

For dissolving of $\left(\mathrm{MgCO}_{3}\right)_{4}{ }^{*} \mathrm{Mg}(\mathrm{OH})_{2}{ }^{*} 5 \mathrm{H}_{2} \mathrm{O}$ titrate the $\mathrm{pH}$ with $\mathrm{HCL}$ until the solution is clear. Afterwards adjust the $\mathrm{pH}$ with $5 \mathrm{M} \mathrm{NaOH}$ to7.8

Ad Luciferin and ATP at last. Control pH

Storage of the Buffer ad $-20^{\circ} \mathrm{C}$ without light, thaw at room temperature

Renilla-luciferase-assay buffer

\begin{tabular}{|c|c|c|c|}
\hline $\begin{array}{l}\mathrm{NaCl} \\
\mathrm{Na}_{2} \text {-EDTA }\end{array}$ & $1,1 \mathrm{M}$ & $32,15 \mathrm{~g}$ & $96,45 \mathrm{~g}$ \\
\hline $\begin{array}{l}\mathrm{Na}_{2} \text {-EDTA } \\
\text { (Disodium ethylenediamine tetr }\end{array}$ & $2,2 \mathrm{mM}$ & $2,2 \mathrm{ml}(0,5 \mathrm{M}) \quad 6,6 \mathrm{r}$ & $6,6 \mathrm{ml}$ \\
\hline $\mathrm{KxPO}_{4}(\mathrm{pH} 5,1)$ & $0,22 \mathrm{M}$ & $110 \mathrm{ml} 1 \mathrm{M} \mathrm{KH}_{2} \mathrm{PO}_{4}$ & $330 \mathrm{ml}$ \\
\hline BSA & $0,44 \mathrm{mg} / \mathrm{ml}$ & $220 \mathrm{mg}$ & $660 \mathrm{mg}$ \\
\hline $\mathrm{NaN}_{3}$ & $1,3 \mathrm{mM}$ & $42,25 \mathrm{mg}$ & $126,75 \mathrm{mg}$ \\
\hline Coelenterazin (in $100 \%$ EtOH) & $1,43 \mathrm{mM}$ & $300 \mu \mathrm{l}$ Stock & $900 \mu \mathrm{l}$ \\
\hline
\end{tabular}

$\mathrm{KxPO}_{4}(\mathrm{pH} 5,1): 1 \mathrm{M} \mathrm{KH}_{2} \mathrm{PO}_{4}$, Adjust the $\mathrm{pH}$ with $\mathrm{KOH}$ to 5.1

add Coelenterazin as last ingredience

Coelenterazin stock is $1 \mathrm{mg}$ in $1 \mathrm{ml} \mathrm{EtOH}$, Storage at $-20^{\circ} \mathrm{C}$

Adjust the $\mathrm{pH}$ of the Buffer to 5.0

Storage of the Buffer ad $-20^{\circ} \mathrm{C}$ without light, thaw at room temperature 
All substrates are from PJK (http://www.pjk-gmbh.de).

D-Luciferin, free acid:

No. 260150

Co-Enzyme A:

No. 260809

ATP:

No. 260920

DTT:

No. 260710

Coelenterazin:

No. 260350

\subsubsection{Solutions for cell culture}

$2 x$ freezing medium for eukaryotic cells

$\begin{array}{ll}\text { DMEM } & 55 \% \\ \text { DMSO } & 20 \% \\ \text { FBS } & 45 \%\end{array}$

PLL 250x

Poly-L-Lysine in ddH2O

f.c. for coating PC12 plates

$5 \mathrm{mg} / \mathrm{ml}$

$0,02 \mathrm{mg} / \mathrm{ml}$

Penicillin-Streptomycin 100x

Potassium Penicillin

Streptomycin Sulfate

$10,000 \mathrm{U} / \mathrm{ml}$

$10,000 \mathrm{ug} / \mathrm{ml}$

Trypsin-EDTA 10x

Trypsin

$5 \mathrm{~g}$

EDTA

$6,85 \mathrm{mM}$

Transfection medium

OptiMEM-Medium (Gibco)

Nutrition media

DMEM $1 \mathrm{~g}$ Glucose/l Lonza phenol red

DMEM 4,5 g Glucose/l Lonza phenol red

DMEM $1 \mathrm{~g}$ Glucose/l Lonza

DMEM 4,5 g Glucose/l Lonza

Horse Serum HS, heat inactivated for $45^{\prime \prime}$ at $56^{\circ} \mathrm{C}$

Fetales Bovines Serum FBS, heat inactivated for $45^{\prime \prime}$ at $56^{\circ} \mathrm{C}$

PenStrep

GlutaMAX-I (stable L alanine-L-glutamine-dipeptide)

$\begin{array}{lllll}\text { Cell line } & \text { Glucose } & \text { Percent FCS } & \text { Percent HS } & \text { PSG } \\ \text { HEK } & 4,5 \% & 10 \% & --- & 1 \% \\ \text { PC12 } & 1 \% & 10 \% & 5 \% & 1 \%\end{array}$

Standard IP buffer (1\% NP40)

For $250 \mathrm{ml}$

$12.5 \mathrm{ml} 1 \mathrm{M}$ Tris $\mathrm{pH} 7.5$

Final concentration []

$7.5 \mathrm{ml} 5 \mathrm{M} \mathrm{NaCl}$

$50 \mathrm{mM}$

$2.5 \mathrm{ml} \mathrm{NP} 40$ (or $25 \mathrm{ml} \mathrm{10 \%} \mathrm{NP40)}$

$150 \mathrm{mM}$

$0.5 \mathrm{ml} 0.5 \mathrm{M}$ EGTA

$1 \%$

$1 \mathrm{mM}$

or Hepes-based Lysis buffer 
Add freshly:

Complete tablet (Roche)(mini for $10 \mathrm{ml}$ )

Phosphatase inhibitor II and III (Sigma): $10 \mu \mathrm{l} / \mathrm{ml}$ each

$\mathrm{NaF}(0.5 \mathrm{M}): 200 \mu \mathrm{l} / \mathrm{ml}$ (f.c. $100 \mathrm{mM}$ )

Optional:

$\mathrm{Na}_{3} \mathrm{VO}_{4}(0.2 \mathrm{M}) ; 5 \mu \mathrm{l} / \mathrm{ml}$ (f.c. $\left.1 \mathrm{mM}\right)$

PMSF $(100 \mathrm{mM}): 10 \mu \mathrm{l} / \mathrm{ml}$

DTT (1M): $10 \mu \mathrm{l} / \mathrm{ml}$

IP buffer for anti-Flag IP (with 1\% Triton-X100):

For $250 \mathrm{ml}$

$12.5 \mathrm{ml} 1 \mathrm{M}$ Tris $\mathrm{pH} 7.5$

$7.5 \mathrm{ml} 5 \mathrm{M} \mathrm{NaCl}$

$2.5 \mathrm{ml}$ Triton-X100 (or $25 \mathrm{ml}$ 10\% Triton-X)

$50 \mathrm{mM}$

$150 \mathrm{mM}$

$1 \%$

$1 \mathrm{mM}$

$0.5 \mathrm{ml} 0.5 \mathrm{M}$ EGTA

$0.5 \mathrm{M}$ EGTA: $50 \mathrm{ml}$ titrate with $5 \mathrm{M} \mathrm{NaOH}$ (ca. $8 \mathrm{ml}$ ), or with $10 \mathrm{M} \mathrm{NaOH}$ ca. $4 \mathrm{ml}$ ).

1X Running buffer:

$3.03 \mathrm{~g}$ Tris

$14.4 \mathrm{~g}$ Glycine

$1 \mathrm{~g}$ SDS

add water to 1 I

1X Transfer buffer:

$3.03 \mathrm{~g}$ Tris

$14.4 \mathrm{~g}$ Glycine

$200 \mathrm{ml}$ Methanol

add water to $1 \mathrm{I}$

Sucrose buffer for brain lysates

$\begin{array}{llll}\text { sucrose } 80 \% & 4^{\circ} \mathrm{C} & 320 \mathrm{mM} & 1,37 \mathrm{ml} \\ 1 \mathrm{M} \text { Tris } & & 10 \mathrm{mM} & 100 \mu \mathrm{l} \\ 2 \mathrm{M} \mathrm{NaHCO} \quad & 4^{\circ} \mathrm{C} & 1 \mathrm{mM} & 5 \mu \mathrm{l} \\ 2 \mathrm{M} \mathrm{MgCl} 2 & 1 \mathrm{mM} & 5 \mu \mathrm{l} \\ \mathrm{H} 2 \mathrm{O} & & 8,134 \mathrm{ml} \\ \text { protease inhibitor } & & 1 \text { tablet } \\ \text { Phosphatase inhib. } & \mathrm{Na} 4 \mathrm{P} 2 \mathrm{O} 7 & 225 \mu \mathrm{l} \\ \left(-20^{\circ} \mathrm{C} \text { freezer) }\right. & \mathrm{NaF} & 200 \mu \mathrm{l} \\ & \mathrm{Na3VO} 4 & 50 \mu \mathrm{l} \\ & \mathrm{ZnCl} 2 & 10 \mu \mathrm{l} \\ \text { total volume } & & 10 \mathrm{ml}\end{array}$

4x LDS sample buffer (NUPAGE)

$40 \%$ glycerol

$564 \mathrm{mM}$ Tris-Base

$4.6 \mathrm{ml} 87 \%$ glycerol

$424 \mathrm{mM}$ Tris-HCl

$0.682 \mathrm{~g}$

$0.666 \mathrm{~g}$

LDS (lithium dodecyl =lauryl sulphate) $\quad 0.8 \mathrm{~g}$

$2.5 \%(\mathrm{v} / \mathrm{v})$ phenol red (1\%solution) 250ul

$7.5 \%(v / v)$ Serva Blue G250 (1\%solution) 750ul

2mM EDTA

40ul 0.5M EDTA or $0.006 \mathrm{~g}$

$1 \mathrm{x}$ buffer should be $\mathrm{pH}$ 8.5. Do not adjust the $\mathrm{pH}$.

2x Freezing medium for mammalian cells

$\begin{array}{lll} & 10 \mathrm{ml} & 15 \mathrm{ml} \\ \text { DMSO }(20 \%) & 2 \mathrm{ml} & 3 \mathrm{ml} \\ \text { FCS }(25 \%) & 2,5 \mathrm{ml} & 3,75 \mathrm{ml} \\ \text { DMEM }(55 \%) & 5,5 \mathrm{ml} & 8,25 \mathrm{ml}\end{array}$




\subsection{The Clinical Collection}

The NIH Clinical Collection (NCC) is a plated array of 281 (NCC201) and 446 (NCC003) small molecules with a history of human clinical trials. The substances are distributed by Molecular Libraries Small Molecule Repository (MLSMR) Evotec San Francisco. The collection was assembled by the American National Institutes of Health $(\mathrm{NIH})$. Similar collections of Food and Drug Administration (FDA) approved drugs have been used to detect undiscovered bioactivity and therapeutic potentials. The aim of the collection is to do likewise, to provide excellent starting points for chemical lead optimisation or even to discover treatment effects in new diseases areas.

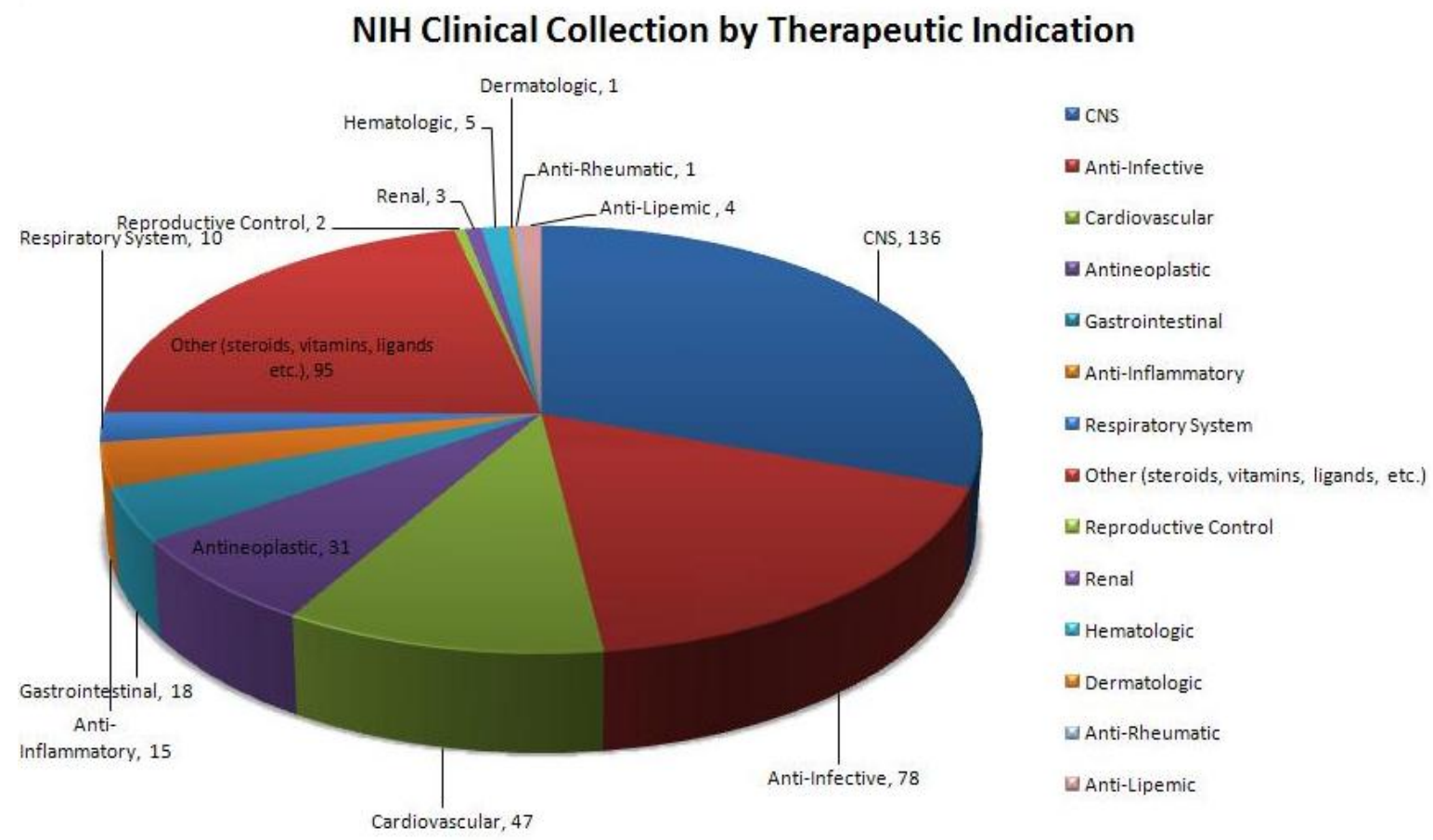

Figure 16: NIH Clinical colection

Image by Evotec

http://www.nihclinicalcollection.com/NCCAnnotatedChartVP.JPG

For the screen all chemicals are solved in DMSO and used in a final concentration of $10 \mu \mathrm{M}$ if possible. All substances were purity checked at 16.11.2007 aliquoted at 10.12.2010 or 29.09.2011. For a detailed list of drugs see apendix. 


\subsection{1}

\subsection{Drugs for validation}

\begin{tabular}{|c|c|c|c|}
\hline Name & Manufacturer & Amount & Order number \\
\hline Lapatinib & Axon & $10 \mathrm{mg}$ & 1395 \\
\hline Cl-1033 & Axon & $5 \mathrm{mg}$ & 1433 \\
\hline Staurosporin & Cayman & $100 \mu \mathrm{g}$ & 81590 \\
\hline SB-431542 hydrate & Sigma & $5 \mathrm{mg}$ & S4317 \\
\hline EGF-like domain & Reprokine & $50 \mu \mathrm{g}$ & RKQ02297 \\
\hline mEGF & Reprokine & $50 \mu \mathrm{g}$ & RKP01132 \\
\hline Rapamycin & Calbiochem & $100 \mu \mathrm{g}$ & 533210 \\
\hline MK-801 (Dizocilpine) & Sigma & $25 \mathrm{mg}$ & M107 \\
\hline Spironolactone & Sigma & $1 G$ & S3378 \\
\hline Eplerenone & Sigma & $10 \mathrm{mg}$ & E6657 \\
\hline Canrenone & Santa Cruz & $1 \mathrm{~g}$ & 205616 \\
\hline Topotecan & Selleckchem & $50 \mathrm{mg}$ & S11231 \\
\hline SN38 & Tocris & $10 \mathrm{mg}$ & 2684 \\
\hline Vincristine & Tocris & $10 \mathrm{mg}$ & 1257 \\
\hline Mevastatin & Sigma & $5 \mathrm{mg}$ & M2537 \\
\hline Albendazole & Sigma & $10 \mathrm{~g}$ & A4673 \\
\hline CCPA & Tocris & $10 \mathrm{mg}$ & 1705 \\
\hline $\mathrm{K} 252 \mathrm{a}$ & LC Laboratories & $1 \mathrm{mg}$ & K-2151 \\
\hline
\end{tabular}

Table 11. Drugs ordered for validation purpose 


\section{Methods}

\subsection{General lab routine}

Methods not described in detail were performed according to "Molecular cloning: A Laboratory Manual" (Maniatis,1982). Molecular biology standard methods were performed according to Sambrook and Russel (Sambrook and Russell, 2001) and to the manufacturer's instructions if not indicated otherwise.

\subsection{Transformation of chemically competent bacteria}

Aliquots of transformation-competent bacteria (E.coli, strain XL1 blue, DH5a, Mach1) were thawed on ice or on the way. 2.5-30 $\mu$ l of a ligation or recombination reaction was added to $50-100 \mu$ l cells (for retransformation of existing plasmids 1ul DNA and 10ul bacteria is sufficient), followed by an incubation step on ice for 5-30 min. The bacteria were heat-shocked at $42^{\circ} \mathrm{C}$ for $30 \mathrm{sec}$, and then cooled on ice for $2 \mathrm{~min}$. $600 \mu \mathrm{l}$ cold LB medium or SOC medium without antibiotics was added, and the mix was incubated at $37^{\circ} \mathrm{C}$ for 45 min without or with moderate shaking. During this incubation the cells start expressing the appropriate antibiotic resistance. Then, $100-800 \mu l$ were plated on pre-warmed LB-agar plates containing the appropriate antibiotics. Sterile glass beads were used to equally distribute the bacteria. The plates were incubated overnight at $30-37^{\circ} \mathrm{C}$ depending on the encoded genes. (Note ERBB constructs should be expressed on not more than $32^{\circ} \mathrm{C}$ ).

\subsection{Electroporation of bacteria}

The commercially available electro-competent E.coli strain $\mathrm{DH} 10 \mathrm{~B}$ was diluted $1: 4$ with sterile $10 \%$ glycerol, and $20 \mu \mathrm{l}$ aliquots were made. For electroporation, the bacteria were thawed on ice, $2-2.5 \mu$ l of a recombination or ligation reaction was added, and the mix was transferred into a $1 \mathrm{~mm}$ electroporation cuvette (BioRad).

Be aware of to high concentrations of salt in the DNA. In case of producing an electric arc dilute the DNA further with the 10\% glycerol. The electroporation was performed using the 'GenePulserll' (BioRad) with the following settings: $1.75 \mathrm{kV}, 25 \mu \mathrm{F}$ capacitance and resistance of $200 \Omega$. The cells were resuspended in $600 \mu \mathrm{l}$ cooled LB, or alternatively in SOC medium, without antibiotics and incubated at $37^{\circ} \mathrm{C}$ for $45 \mathrm{~min}$ with moderate shaking. The bacteria were plated as described above in the section 'Transformation of chemically competent bacteria'. 


\subsection{Plasmid purification}

Plasmid DNA preparations are based on a modified protocol of Birnboim and Doly (Birnboim and Doly, 1979), depending on the alkaline cell lysis followed by SDS precipitation of proteins and genomic DNA. The plasmid DNA was bound to a silica membrane under appropriate high-salt conditions, washed and finally eluted with buffer TE or $\mathrm{H}_{2} \mathrm{O}$. Plasmid DNA was isolated from fresh overnight bacterial LB cultures grown to an OD of 2 to 3 using the Plasmid DNA Purification Kits (Mini, Midi, Maxi, Maxi EF and Giga scale) provided by Macherey-Nagel. Detailed description of the procedures can be found in the manuals provided by the manufacturers.

\subsection{Plasmid DNA mini preparations}

In brief, the pelleted bacteria (2-4 $\mathrm{ml}$ of the overnight culture) were resuspended in $250 \mu \mathrm{l}$ buffer A1 with RNAse. Cell lysis was performed by adding $250 \mu \mathrm{l}$ lysis buffer A2. After 5 min incubation the reaction was stopped by adding $300 \mu \mathrm{l}$ neutralization buffer $\mathrm{A} 3$. The mix was centrifuged for $10 \mathrm{~min}$ to pellet genomic DNA as well as proteins, and the clear supernatant was then loaded onto the column. The resin membrane was washed with $600 \mu \mathrm{l}$ buffer $A Q$, dried by centrifugation for $2 \mathrm{~min}$, and finally, the plasmid DNA was eluted in $100 \mu \mathrm{H}_{2} \mathrm{O}$ or TE buffer.

\subsection{Plasmid DNA midi preparations}

In brief, pelleted bacteria $(200 \mathrm{ml}$ of the overnight culture) were resuspended in $4 \mathrm{ml}$ buffer $\mathrm{S} 1$ with RNAse. $4 \mathrm{ml}$ buffer $\mathrm{A} 2$ was added to lyse the cells and after $5 \mathrm{~min}$ incubation at RT cell lysis was stopped by adding $4 \mathrm{ml}$ buffer S3 pre-chilled to $4^{\circ} \mathrm{C}$. The suspension was incubated on ice for $5-15 \mathrm{~min}$. In the meantime, the column was equilibrated with $2.5 \mathrm{ml}$ buffer $\mathrm{N} 2$. The cleared and filtered lysate was loaded on the column. The column was washed twice with $10 \mathrm{ml}$ buffer $\mathrm{N} 3$, and the DNA was eluted in $5 \mathrm{ml}$ buffer N5. Finally, the DNA was precipitated using $35 \%$ f.c. isopropanol, and it was re-suspended in 100 $\mu \mathrm{l}$ TE buffer. The expected amount of plasmids is $200-500 \mu \mathrm{g}$. The f.c. in stock is adjusted to $1 \mu \mathrm{g} / \mu \mathrm{l}$.

\subsection{Photometric concentration determination of nuclear acids}

Concentration and purity of a nucleic acids solution can be analysed by spectrophotometry. According to the Lambert-Beer law, the concentration of a solution is directly proportional to its extinction or absorption, that is $A=\varepsilon^{*} C^{*}$

with $\varepsilon$ representing the molar extinction coefficient (unit $M-1 \mathrm{~cm}-1$ ), c being the 
concentration (unit M) and I being the optic path length (cuvette thickness) that the light passes through the sample (unit $\mathrm{cm}$ ). The extinction coefficients for nuclear acids at $\lambda=260 \mathrm{~nm}$ are:

$$
\begin{array}{rlr}
\text { guanine: } & \varepsilon=12010 & \mathrm{M}-1 \mathrm{~cm}-1 \\
\text { cytosine: } & \varepsilon=7050 & \mathrm{M}-1 \mathrm{~cm}-1 \\
\text { adenine: } & \varepsilon=15200 & \mathrm{M}-1 \mathrm{~cm}-1 \\
\text { thymine: } & \varepsilon=8400 & \mathrm{M}-1 \mathrm{~cm}-1 \\
\text { (uracile: } & \varepsilon=8111 & \mathrm{M}-1 \mathrm{~cm}-1 \text { ) }
\end{array}
$$

Using a spectrophotometer, the absorption at 260nm (maximum of absorption of nucleic acids) and $280 \mathrm{~nm}$ (maximum of absorption of aromatic amino acids in proteins) of the nucleic acid solution was determined. For a reliable measurement, it is of importance that the value for the absorption at $260 \mathrm{~nm}$ is set between 0.1 and 1 to fit the linear range of the photometer. Therefore, a proper dilution of the sample was necessary. To determine the concentration the following relation was applied:

1 OD260 (optical density at $260 \mathrm{~nm}$ ) $=50 \mathrm{mg} / \mathrm{ml}$ for double stranded (ds) DNA

1 OD260 $=40 \mathrm{mg} / \mathrm{ml}$ for single stranded (ss) RNA

1 OD260 $=33 \mathrm{mg} / \mathrm{ml}$ for ss oligonucleotides

The equation to determine the concentration of dsDNA was

$c(\mu \mathrm{g} / \mu \mathrm{l})=\mathrm{OD} 260 * 50 *$ dilution factor $/ 1000$

The purity of a given sample was assessed by the relative absorption values of $260 \mathrm{~nm}$ over $280 \mathrm{~nm}$. Clean preparations of DNA and RNA would return values between 1.8 and 2.0 whereas significantly lower values indicated a protein contamination.

\subsection{Agarose gel electrophoresis}

Agarose gels of $0.8 \%-4 \%$ were used to separate DNA fragments between $0.1 \mathrm{~kb}$ and $15 \mathrm{~kb}$ of size. The proper amount of agarose was added to $1 \times$ TAE buffer, and the suspension was heated, in the microwave, for appr. $5 \mathrm{~min}$, or longer, until the agarose was completely dissolved. In case of ethidium bromide gels the solution was allowed to cool to $60^{\circ} \mathrm{C}$ to minimise evaporation of gases, and ethidium bromide was added to a final concentration of $1 \mu \mathrm{g} / \mu \mathrm{\mu}$. Be aware of ethidium bromide evaporating in to hot agarose. Don't breath the fumes!

In case of acrydin orange nothing is added to the agarose

The fluid agarose was poured into a gel-casting form, and combs were 
inserted to define the pockets for sample loading. The agarose needed 20$120 \mathrm{~min}$ to solidify depending on the room temperature. Solid agaros gel is storable for 4 weeks at $4^{\circ} \mathrm{C}$. The gel was placed in a chamber containing $1 \mathrm{x}$ TAE buffer. The DNA samples were prepared by adding the proper volume of 10x loading dye (ethidium bromide gel) or 10x OrangeG and GelRed containing loading dye (ethidium bromide free gels) and loaded into the pockets of the gel. The voltage applied was $30-180 \mathrm{~V}(5-10 \mathrm{~V} / \mathrm{cm})$, depending on the size of the gel chamber and the agarose concentration used. For $1.5 \%$ agarose gel to analyse restriction digests the following settings were used:

$\begin{array}{ll}\text { small } & 120 \mathrm{~V} \\ \text { medium } & 150 \mathrm{~V} \\ \text { large } & 180 \mathrm{~V}\end{array}$

DNA molecules migrate to the positive electrode as the phosphates in the DNA backbones are negatively charged. The speed of the migration is lowered by the size of the DNA molecule and the obstruction by the fishnet like structure of the agarose gel, resulting in their size-dependent separation. The DNA could be visualised by the fluorescence of the incorporated ethidium bromide when exposed to UV light source emitting $260 \mathrm{~nm}$.

OrangeG could be visualized via a BioDoc Analyzer

The 100bp marker or $1 \mathrm{~kb}$ marker (Fermentas, St. Leon-Rot) were used as molecular size standards. Gel chamber, casting form and combs were produced by the workshop of the Max-Planck-Institute.

\subsection{Isolation of DNA from agarose gels}

To isolate DNA from agarose gels the Gel and PCR clean up kit provided by Macherey-Nagel was used. The area of the gel containing the desired DNA was excised under UV light (356nm, Intas UV systems, Heidelberg) with a scalpel. The step should be as fast as possible, cause UV light will crosslink DNA and therefore cause mutations. The excised Gel was weighted and transferred to an Eppendorf reaction tube. The DNA was purified according to the manufacturer's instructions. In brief, for each $100 \mathrm{mg}$ of agarose gel, $200 \mu \mathrm{l}$ of buffer NTI was added. The mix was incubated for $5-20 \mathrm{~min}$ at $50^{\circ} \mathrm{C}$ until the agarose was dissolved completely. The sample was loaded onto a silica

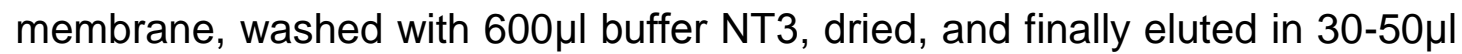
buffer NE prewarmed to $60^{\circ} \mathrm{C}$. Concentration and size of the DNA was then checked via agarose gel electrophoresis. 


\subsection{DNA digest with restriction endonucleases}

DNA restriction digests were performed using type II restriction

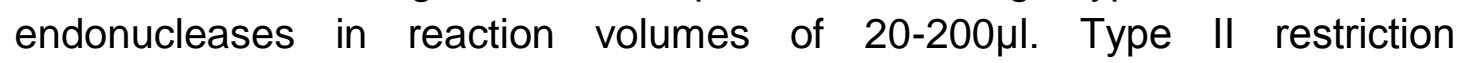
endonucleases induce cleavage mostly within their palindromic recognition sequences of 4-8 nucleotides, and yield in $5^{\prime}$ - or $3^{\prime}$-DNA overhangs or blunt ends depending on the enzyme. Most enzymes were supplied with one of four standard 10x reaction buffers (NEB, New England Biolabs). 0.5 to $1 \mu \mathrm{g}$ of DNA were used for analytical digests, $5-25 \mu \mathrm{g}$ of DNA were used for preparative digests, respectively. The restriction enzyme unit ' $U$ ' defines the amount of enzyme that is required to cut $1 \mu \mathrm{g}$ DNA in 1 hour at $37^{\circ} \mathrm{C}$. Usually, 2- to 3 -fold the amount of enzyme was used in digests and the digests were incubated for two hours. To save enzymes larger digests were incubated for longer than two hours e.g. overnight.

\subsection{DNA sequencing}

DNA sequencing was performed at the Institute's DNA Core Facility (Department of Neurobiology, MPI of Experimental Medicine, Göttingen) lead by Dr. Fritz Benseler. DNA sequencing is based on a modified dideoxy chain reaction termination method according to Sanger (Sanger et al., 1977). The approach is based on a linear DNA amplification in the presence of a sequencing primer, a DNA-polymerase, normal deoxynucleoside triphosphates (dNTPs), and modified nucleotides (dideoxyNTPs) that terminate DNA strand elongation. DideoxyNTPs stochastically incorporate into the newly synthesized DNA by replacing the normal dNTPs thereby terminating the synthesis. The products of different length are separated by capillary electrophoresis. As each of the four dideoxyNTPs is coupled to a different fluorescent dye the DNA fragments can be characterised by size, and the terminal nucleotide is identified. Through a repetitive procedure, the DNA sequence is determined.

\subsection{Sequence analysis of DNA}

Sequencing data was analysed using the DNAstar software, version 8, provided by Lasergene. The obtained sequences were also aligned to databases of the 'National Center for Biotechnology Information' (NCBI, http://www.ncbi.nlm.nih.gov) and ENSEMBL (http://www.ensembl.org).

\subsection{Modification of DNA}

Ligation of DNA The enzyme DNA ligase derived from the bacteriophage T4 catalises the formation of covalent phosphodiester bonds between free 3'hydroxy and 5'-phosphate overhangs of double stranded DNA. Cofactors 
needed for this reaction are $\mathrm{Mg}^{2+}$ ions and ATP. The cloning of DNA fragments, that is the ligation of insert and vector, was performed for $2 \mathrm{~h}$ at room temperature or overnight at $4^{\circ} \mathrm{C}$. The $10-20 \mu$ l of a ligation reaction contained 50-100ng vector DNA, 150-300ng insert DNA (molar ratio 1:3), 1.5U T4 DNA-ligase, and the ATP-containing 10x buffer provided by the manufacturer. (Note: ATP decays over time. If the Ligation is not working ATP is probably gone). The reaction was stopped by incubation at $65^{\circ} \mathrm{C}$ for $10 \mathrm{~min}$. Standard ligation reaction:

$\begin{array}{ll}x \mu \mathrm{l} & \text { vector } \quad(50-100 \mathrm{ng} \text { DNA }) \\ \mathrm{x} \mu \mathrm{l} & \text { insert } \quad(150-300 \mathrm{ng} \mathrm{DNA}) \\ 1 \mu \mathrm{l} & 10 \mathrm{x} \text { buffer } \\ 0.5 \mu \mathrm{l} & \text { T4 ligase }(1.5 \mathrm{U})\end{array}$

Add up to $10 \mu \mathrm{lddH} 2 \mathrm{O}$

\subsection{Dephosphorylation of 5'-DNA fragment overhangs, vectors only}

The terminal 5'-phosphate groups of dsDNA can be removed by phosphatase treatment using the enzyme calf intestinal alkaline phosphatase (CIP). This helps to reduce the likelihood of unwanted vector re-ligations. As CIP is compatible with NEB-, Roche- and Promega-buffers $1-2 \mu$ of the enzyme was added to a completed DNA digest and incubated at $37^{\circ} \mathrm{C}$ for maximal $20 \mathrm{~min}$. To stop the reaction the digested DNA fragment was heat inactivated and/or purified by agarose electrophoresis.

\subsection{Cloning of PCR products}

PCR products were purified by agarose electrophoresis and using the Gel and PCR clean up kit (Macherey-Nagel). The PCR products were, if they contained terminal restrictions sites, first cut with the appropriate restriction enzyme(s), purified, and then used for ligation into the designated vector. PCR products amplified with Taq-polymerase were also directly sub- cloned into the pGemT or pGemTeasy vector (Promega) through so-called TA cloning. The technique is based on the property of the Taq-polymerase to attach an additional nucleotide (preferentially adenosine) to the 3'-end of the completed PCR product. The pGemT vectors can be purchased in a linearised form with complementary 5 'thymine overhangs which bind to the PCR fragment. If a proofreading polymerase with 3'-exonuclease activity (e.g. Pfu-polymerase, Stratagene) was used in the PCR, the adenosine overhangs had to be added in a second reaction using the Taq-polymerase. To do this, $25 \mu \mathrm{l}$ of the purified PCR product were incubated with $5 \mu \mathrm{l} 10 \mathrm{x}$ buffer, dATP (f.c. $0.2 \mathrm{mM}$ ), and $5 \mathrm{U}$ Red Taq-polymerase in a final reaction volume of $50 \mu \mathrm{l}$ 
incubated for $10 \mathrm{~min}$ at $37^{\circ} \mathrm{C}$. The reaction was purified with the help of the

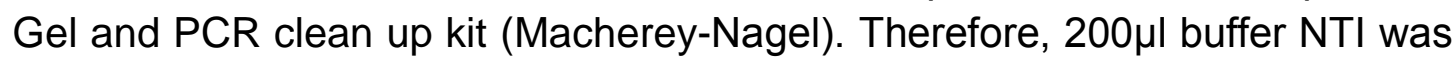
added per $100 \mu \mathrm{I}$ PCR reaction volume, the mix was applied onto the column,

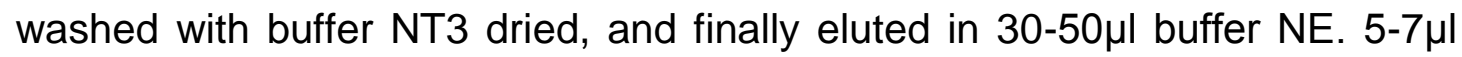
was used for ligation.

\subsection{Amplification of DNA by polymerase chain reaction}

The polymerase chain reaction (PCR) was developed by Kary Banks Mullis (Mullis et al., 1992; Mullis and Faloona, 1987), and is now a commonly used method for selective in vitro amplification of defined DNA fragments. The DNA synthesis begins with the annealing of two DNA oligonucleotides (primers), which flank the DNA stretch that is supposed to be amplified. One primer anneals as a sense primer to the plus strand of the DNA, the other primer binds as an anti-sense primer to the minus strand. The polymerase recognises the dsDNA (primer and template) and exponentially amplifies the DNA region flanked by the primers. The synthesis is catalysed by thermostable Taq-polymerases (derived from the thermophilic bacterium Thermus aquaticus) or proofreading polymerases (e.g. Pfu-polymerase, Stratagene, derived from the thermophilic archaea bacterium Pyrococcus furiosus). The latter one helps to reduce the mutation rate by its 5'-3'exonuclease activity by one power to about one mutation in 106 nucleotides.

\section{Standard PCR reaction $(20 \mu \mathrm{l})$ :}

$x \mu$ template (10-100ng)

$1 \mu \mathrm{l}$ sense (forward) primer $(10 \mathrm{pmol} / \mu \mathrm{l})$

$1 \mu \mathrm{l}$ anti-sense (reverse) primer $(10 \mathrm{pmol} / \mu \mathrm{l})$

$2 \mu \mathrm{l} 10 \mathrm{x}$ reaction buffer (including $\mathrm{MgCl} 2$ )

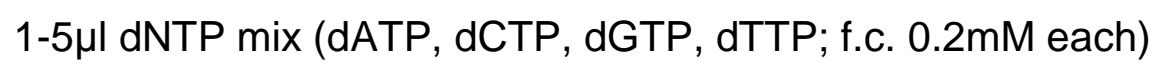

$0.8 \mu \mathrm{l}$ Taq polymerase $(5 \mathrm{U} / \mu \mathrm{l})$ Add ddH2O to $20 \mu \mathrm{l}$

A PCR reaction consists of three main steps:

1. denaturation,

2. primer annealing

3. elongation.

The PCR steps are run at different temperatures, and they are repeated multiple times in cycles. At the beginning of the PCR, an initial denaturation at $95^{\circ} \mathrm{C}$ was used to remove any DNA secondary structures followed by a primer annealing step between $50-60^{\circ} \mathrm{C}$ where the primers are supposed to bind to their complementary DNA sequence. The annealing temperature was $2-4^{\circ} \mathrm{C}$ 
lower than the computed melting temperature of the primers. At $72^{\circ} \mathrm{C}$ the elongation was performed, which is the optimum temperature for the Taqpolymerase. The extension time was calculated depending on the template length and the polymerase used. Non proofreading polymerases require up to $30 \mathrm{sec}$ per $1 \mathrm{~kb}$ template, whereas proofreading enzymes need about $1 \mathrm{~min}$ per $1 \mathrm{~kb}$. The PCR ended in a final elongation step at $72^{\circ} \mathrm{C}$ to ensure complete synthesis. Depending on the DNA template and the purpose (cDNA, plasmid DNA, genomic DNA) 15-36 cycles were run. The volume of the PCR reaction was $20 \mu \mathrm{l}$ (genotyping) or 50 $\mathrm{\mu l}$ (preparative PCR).

\section{Standard PCR protocol}

3 min $\rightarrow 95^{\circ} \mathrm{C}$, initial denaturation

$30 \mathrm{sec} \rightarrow 56^{\circ} \mathrm{C}$, annealing

$60 \mathrm{sec} \rightarrow 72^{\circ} \mathrm{C}$, elongation

$30 \mathrm{sec} \rightarrow 95^{\circ} \mathrm{C}$, denaturation

$60 \mathrm{sec} \rightarrow 55^{\circ} \mathrm{C}$, final annealing

$10 \mathrm{~min} \rightarrow 72^{\circ} \mathrm{C}$, final elongation cycle

\section{Designing primer sequences}

Primers were designed with melting temperatures $\left(T_{m}\right)$ of $60^{\circ} \mathrm{C}$. Primer melting temperature can be roughly calculated with the help of the following formula: $T_{m}=(A+T) 2+(G+C) 4$, with $A, T, G$ and $C$ indicating the numbers of the corresponding nucleotides in the primer sequence. For a more precise $T_{m}$ determination, an online algorithm developed by Warren A. Kibbe (Kibbe, 2007) was used (http://www.basic.northwestern.edu/biotools/oligocalc.html).

\section{Site-directed mutagenesis}

This method of PCR-based in vitro mutagenesis presents a fast approach to exchange and manipulate one or more nucleotides, also simultaneously, in a DNA molecule. Following aspects for the design of the mutagenesis oligonucleotides have to be considered: The length of the oligonucleotide is supposed to be in the range between $24 \mathrm{bp}$ and $45 \mathrm{bp}$.

The $T_{m}$ of the oligonucleotide should be higher than $78^{\circ} \mathrm{C}$ and is calculated by using the following formula: $\mathrm{T}_{\mathrm{m}}=81.5+0.41$ ( $\left.\% \mathrm{GC}\right)-675 / \mathrm{N}-\%$ mismatch (with $\mathrm{N}$ being the length of the oligonucleotide in bp and \% GC reflecting the $\mathrm{GC}$ content in per cent).

The mutation, deletion or insertion should preferentially lie in the middle the oligonucleotide and flanked by each side with $10-15 \mathrm{bp}$ which exhibit $100 \%$ complementarity to the target sequence The GC content of the 
oligonucleotide should not be below $40 \%$.

The oligonucleotide should start and end with at minimum one G or C.

The mutagenesis PCRs are exclusively performed using proofreading polymerases. The PCR product was digested with Dpnl, a restriction endonuclease, which specifically recognises the methylated sequence 5'Gm6ATC 3', and therefore cuts only the parental template DNA generated in E.coli. The mutated sample was gel purified and transformed into bacteria for amplification.

\subsection{One-way gateway}

The gateway recombination system is based on site-directed recombination and uses enzymes derived from the bacteriophage lambda that are able to transfer DNA fragments between vectors (Hartley et al., 2000) This cloning strategy works with much higher efficiency than the conventional cloning technique that is based on type II endonucleases and T4 DNA- ligase. For detailed information on gateway cloning see Invitrogen's web page (http://de.invitrogen.com/site/de/de/home/ProductsandServices/Applications/C loning/Gateway- Cloning.html). To clone an ORF or other DNA fragments of interest specific recombination sites (attB1 and attB2) have to be added to both ends via PCR. The PCR product was recombined in a 'BP reaction' with a 'donor' plasmid, termed pDONR, containing attP1 and attP2 recognition sites. The BP reaction is catalised by the Gateway BP clonase II enzyme mix.

\section{Each BP reaction contained:}

$\begin{array}{lll}3 \mu \mathrm{l} & \text { attB-PCR product } & (10-100 \mathrm{ng} / \mu \mathrm{l}) \\ 1 \mu \mathrm{l} & \text { pDONR } & (100 \mathrm{ng} / \mu \mathrm{l}) \\ 1 \mu \mathrm{l} & \text { BP clonase II } & \end{array}$

$\mathrm{BP}$ reactions were incubated overnight at $\mathrm{RT}$. To optimize conditions for transformation, $1 \mu \mathrm{l}$ of proteinase $\mathrm{K}$ was added and incubated at $37^{\circ} \mathrm{C}$ for 10 min, followed by a heat-inactivation step at $95^{\circ} \mathrm{C}$ for $10 \mathrm{~min} .1 \mu \mathrm{l}$ of the inactivated BP reactions was taken for electroporation in $\mathrm{DH} 10 \mathrm{~b}$ bacteria or for chemical transformation in XL1blue bacteria. Clones were selected by the appropriate antibiotic, and the insert was sequenced.

One major feature of the gateway system is that bacteria harbouring a not recombined donor vector after a recombination reaction are not supposed to survive as the pDONR vector contains the toxic gyrase inhibitor encoded by the $\mathrm{ccdB}$ gene flanked by the recombination sites. The $\mathrm{ccdB}$ gene is only replaced by an insert when the recombination event was successful, ensuring that only properly recombined clones survive and produce colonies. 
Constructs containing the pDONR backbone and the DNA insert represent 'entry clones' ( $p E N T R$ ). These constructs carry attL sites (the product of recombination of attB and attP sites). To generate the final 'expression clones', abbreviated $\mathrm{pEXPR}$, the attL sites of the Entry clones are recombined with attR sites of a 'destination vector', or $\mathrm{PDEST}$, in an 'LR' reaction, catalysed by the Gateway LR Clonase II enzyme mix.

\section{Each LR reaction included:}

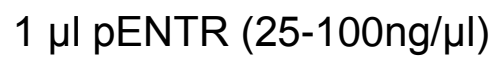

$1 \mu \mathrm{l}$ pDEST $(100 \mathrm{ng} / \mu \mathrm{l})$

$1 \mu \mathrm{L}$ LR clonase

$2 \mu \mathrm{H} 2 \mathrm{O}$

The reaction was incubated at $\mathrm{RT}$ overnight followed by a proteinase $\mathrm{K}$ digest. $1 \mu \mathrm{l}$ of the reaction was transformed into $\mathrm{DH} 10 \mathrm{~b}$ electro-competent or $\mathrm{DH} 5 \alpha$ chemical competent bacteria. The resulting pEXPR clones were then analysed by restriction digest and sequencing and functionally tested by transient transfection in cell culture followed by luciferase assays, fluorescence assays or western blotting.

\subsection{Subculturing of eukaryotic cells}

Eukaryotic cells (HEK293, HEK293FT, PC12, etc) were cultivated on $15 \mathrm{~cm}$ dishes or cell culture flasks at $37^{\circ} \mathrm{C}$ in a humidified incubator containing $5 \%$ CO2. After reaching $70-90 \%$ confluence, cells were subcultured by washing with PBS and mild treatment with $2.5 \mathrm{ml}$ trypsin/EDTA for $1-5 \mathrm{~min}$ at $37^{\circ} \mathrm{C}$ until they detached from the culture dish. The reaction was stopped by adding $5 \mathrm{ml}$ serum-containing culture medium, the cells were resuspended and the cells were pelleted by centrifugation for $3 \mathrm{~min}$ at $800 \mathrm{~g}$. The supernatant was discarded, the pellet resuspended in fresh medium, and an appropriate inoculum (typically, a dilution of $1 / 10$ to $1 / 5$ ) was plated onto new dishes.

\subsection{Thawing and cryopreservation of cell lines}

Frozen stocks of cell lines were kept at $-196^{\circ} \mathrm{C}$ submerged in liquid nitrogen in $2 \mathrm{ml}$ cryovials. Aliquots were quickly thawed in a water bath at $37^{\circ} \mathrm{C}$, suspended with $3 \mathrm{ml}$ pre-warmed growth medium, and centrifuged for $3 \mathrm{~min}$ at $800 \mathrm{~g}$ to remove the DMSO with the supernatant. The pellet was resuspended in fresh medium and plated onto a $15 \mathrm{~cm}$ culture dish.

For cryopreservation cells were grown on $15 \mathrm{~cm}$ dishes until reaching $90 \%$ confluency. The medium was removed, and the cells were detached using trypsin as described above. The cells were counted using a Neubauer 
counting chamber, and, after centrifugation, resuspended in an appropriate volume of ice-cold growth medium to obtain a concentration of about 4 million cells per $1 \mathrm{ml} .500 \mu \mathrm{l}$ of the cell suspension was transferred into a cryovial already containing an equal volume of the ice-cold $2 x$ freezing medium.

The samples were mixed gently by inverting the tubes three times and quickly frozen at $-20^{\circ} \mathrm{C}$. After two hours the cells were transferred to $-80^{\circ} \mathrm{C}$ for one day and finally to liquid nitrogen for long term storage.

\subsection{Coating with poly-L-lysin}

For PC12 cells, it is important to coat the dishes with poly-L-lysin (PLL). The negatively charged polysaccharid structures on the cell surface bind to the positively charged PLL allowing better adherence of the cells. For coating, dishes were incubated with $0.02 \mathrm{mg} / \mathrm{ml} \mathrm{PLL}$ in ddH2O for 20 min at RT, then washed twice with sterile water, air-dried, and stored at $4^{\circ} \mathrm{C}$ until further use.

96 well plates were coated using the Hamilton Lab Star robot. 30 $\mu$ l of PLL solution were pipetted in each well. The PLL solution incubated for 20". Afterwards the wells were washed twice with $\mathrm{ddH} 2 \mathrm{O}$. The coated plates were sterilized via UV light for $1 \mathrm{~h}$

\subsection{Transfection of mammalian cells}

DNA-transfer in eukaryotic cells with lipofectamine2000

The transient transfection of plasmid DNA in eukaryotic cells was done using lipofectamine2000 (Invitrogen). The approach is based on the formation DNAcontaining liposomes that are endocytosed by the cell. Cells were typically transfected in 96-well plates for reporter gene assays. Depending on the cell line different numbers of cells were plated:

$\begin{array}{llll}\text { Cell line } & \text { 96well } & \text { 6well } & \mathbf{1 5 c m} \\ \text { PC12TO } & 40.000 & 1.000 .000 & 5 \mathrm{M} \\ \text { PC12WT } & 40.000 & 1.000 .000 & \\ \text { PC12 Neuroscreen } & 50.000 & & \\ \text { HEK293 } & 20.000 & 500.000 & 5 \mathrm{M} \\ \text { HEK293FT } & & & 5 \mathrm{M}\end{array}$

The protocol provided by the manufacturer was modified to obtain optimal results. For a single well of a 96-well plate, both the 10-200ng (mini or midi prep) of DNA and $0.2 \mu \mathrm{l}$ of lipofactamine2000 were diluted in $15 \mu \mathrm{l}$ OptiMEM each. The solutions were mixed separately, incubated for $5 \mathrm{~min}$, and then 
combined, resulting in $30 \mu \mathrm{l}$. The mix was vortexed for $20 \mathrm{sec}$. and incubated for $20 \mathrm{~min}$ at RT. The growth medium was completely removed from the cells, and $30 \mu \mathrm{l}$ of the OptiMEM containing DNA/lipofectamine complexes were applied onto the cells. After two hours, $30 \mu \mathrm{l}$ of regular growth medium was added to each well. The cells were allowed to express the plasmids for 24$48 \mathrm{~h}$, and subjected to a given procedure, i.e. western blots or reporter gene assays.

\subsection{DNA transfer in eukaryotic cells with electroporation}

During electroporation a brief current pulse induces a transmembrane electric field in the cells, which leads to local instabilities of the cell membrane (electro-pores). In this short period macromolecules such as DNA are able to enter the cell. With optimal setting of the parameters, cells recover and can be subcultured. For the generation of stably transfected Hek293 cells, 5M cells were mixed with $20 \mu \mathrm{g}$ linearised plasmid and transferred into an electroporation cuvette $(4 \mathrm{~mm})$.

Parameters for electroporation were the following: 950 $\mathrm{F}, 220 \mathrm{~V}$. After electroporation, cells were incubated under standard conditions.

\subsection{Generation of stable cell lines}

Cells were transfected and selected with the indicated drug selection concentrations corresponding to the repsective resitance encoded on the expression plasmid.

\section{Hek293}

Puromycin 200ng/ml

Hygromycin $\quad 100 \mu \mathrm{g} / \mathrm{ml}$ selection $\quad 50 \mu \mathrm{g} / \mathrm{ml}$ maintaining

G418 $\quad 400 \mu \mathrm{g} / \mathrm{ml}$ selection $\quad 200 \mu \mathrm{g} / \mathrm{ml}$ maintaining

Hygromycin Co integration

Vector MSCV Hygro Hygromycin $50 \mu \mathrm{g} / \mathrm{ml} \quad 1-5 ; 000,000$ cells

$2 \mu \mathrm{g}$ pMSCV Hygro $\quad 10 \mu \mathrm{g}$ Plasmid of interest linearised

Electroporate the cells and plate the cells on a PLL coated cell culture Plate

Start the selection $24 \mathrm{~h}$ after transfection. After 5 days nearly all cells are dead

After 2 weeks positive survival clones should be visible by eyesight Alternatively, transfection via Lipofectamin 2000 (Invitrogen cat.-no.: 11668019) It isn't necessary to linearize the plasmid of interest 
Transfection scheme for a $10 \mathrm{~cm}$ dish:

\begin{tabular}{|l|l|l|l|}
\hline & O-MEM & LF2000 & DNA \\
\hline DNA tube: & $1,5 \mathrm{ml}$ & $20 \mu \mathrm{l} \mathrm{-}$ & $1,2 \mu \mathrm{g}$ \\
\hline
\end{tabular}

Add the OptiMEM to both tubes; mix well through pipetting and incubate $5 \mathrm{~min}$ at room-temperature (RT). Mix the LF/OptiMEM mixture and the DNA/OptiMEM, incubate $20 \mathrm{~min}$ at RT. Change the medium with $3 \mathrm{ml}$ of LF/DNA-mix per dish, incubate for $1-2 \mathrm{~h}$ at $37^{\circ} \mathrm{C}$, then add $3 \mathrm{ml}$ of fresh $2 \mathrm{x}$ growth medium. Start selection $24 \mathrm{~h}$ after transfection

\section{PC12TO}

Hygromycin Co integration

Vector MSCV-hygro Hygromycin $200 \mu \mathrm{g} / \mathrm{ml} \quad 1-5,000,000$ cells

$2 \mu \mathrm{g}$ pMSCV Hygro $10 \mu \mathrm{g}$ Plasmid of interest linearised

$50 \mu \mathrm{g} / \mathrm{ml} \mathrm{Hygro} \mathrm{is} \mathrm{not} \mathrm{enough!}$

\section{Important for freezing and thawing of stable cells:}

When you freeze the cells change $3 \mathrm{~h}$ before freezing the growth-media to media without selection antibiotics. For freezing use a freezing medium with the double amount of serum at least. Don't forget the DMSO. When thawing let the cells grow for $24 \mathrm{~h}$ in a growth medium without selection antibiotics. After $24 \mathrm{~h}$ you can add the selection antibiotics.

\subsection{Luciferase reporter gene assays}

\subsubsection{Single assay $16 \times 6$ measurement points}

Luciferases are enzymes which catalytic activity leads to the emission of visible light upon substrate conversion. The most commonly used luciferase is the firefly luciferase that catalyses the following reaction:

luciferin + ATP $\rightarrow$ luciferyl adenylate + PPi

luciferyl adenylate $+\mathrm{O} 2 \rightarrow$ oxyluciferin + AMP + light

Transcriptional activity of a given promoter can be investigated by transfecting the cells with a reporter construct carrying a luciferase ORF under the control of this particular promoter. Expression level, of the luciferase reporter gene, is then quantified by adding luciferase substrate and ATP to the cellular lysates and measuring the intensity of the emitted light. Luciferase reporter gene assays were performed in HEK293 cells and PC12 cells in 96-well plates. 12$48 \mathrm{~h}$ after transfection the medium was removed and 30-40 $\mu \mathrm{l}$ of $1 \mathrm{x}$ passive 
lysis buffer (Promega) was added to each well. The plates were incubated for $20 \mathrm{~min}$ at RT under moderate or fast shaking $(200-1400 \mathrm{rpm})$ and assayed immediately, stored at $4^{\circ} \mathrm{C}$ up to $6 \mathrm{~h}$ or frozen at $-20^{\circ} \mathrm{C}$. Prior to analysis the samples were transferred to black, white or black and white plastic microtiter plates. White wells increase the signal. Black plates decrease the noise. Black and white plates combine both features.
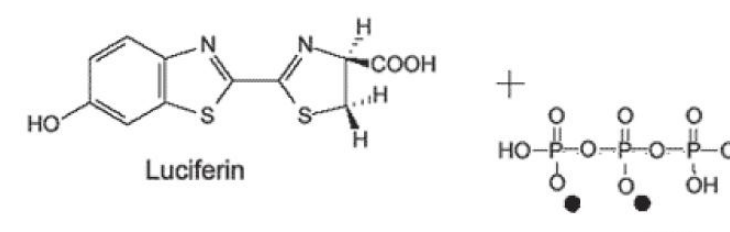

ATP

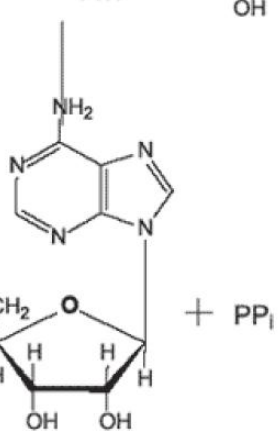

Adenyl-luciferin

Reaction 2
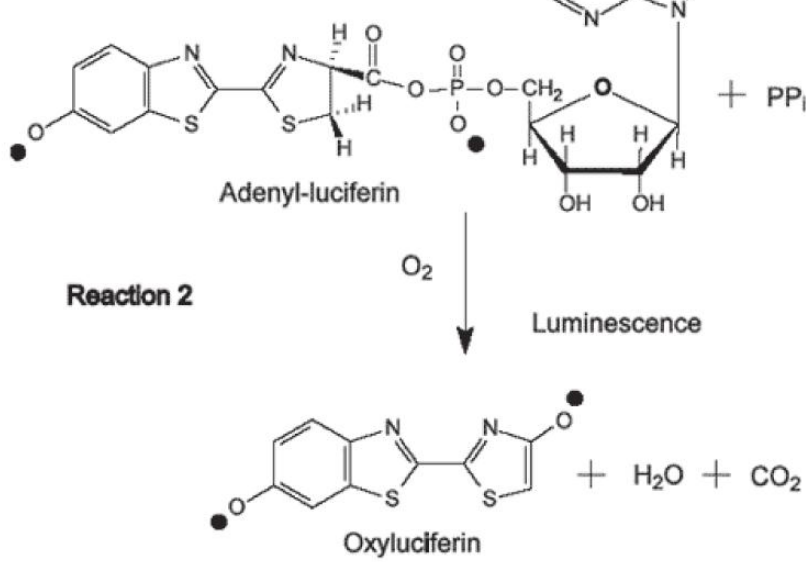

Figure 17: Reaction of D-Luciferine with the firefly luciferase (modified from sigmaaldrich.com)

\subsubsection{Normalisation and transfection control}

Measurements of the firefly luciferase (Photinus pyralis) activity are commonly normalized to the activity of a different reporter, e.g. Renilla luciferase (Renilla reniformis) that is expressed under a constitutively active promoter. This is done to rule out differences in cell number, transfection efficiency, and RNA and protein expression from well to well. 


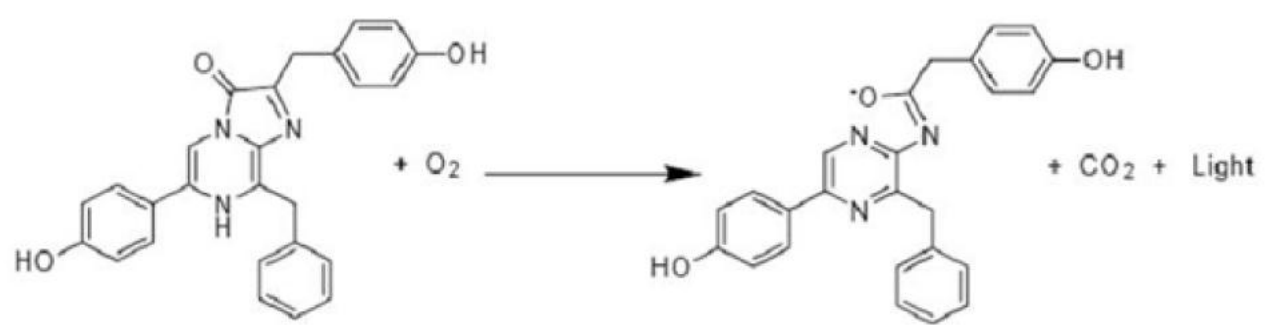

Figure 18: Reaction of Coelenterazine with the Renilla luciferase (modified from Komabiotech.co.kr).

A mix of three different plasmids encoding Renilla luciferas under control of different promoters was used. To visually control transfection efficiency an equal amount of pEYFPnuc, a construct for CMV promoter-driven expression of the nuclear localized enhanced yellow fluorescent protein was used.

$\begin{array}{lll}\text { TC34 } & \text { SV40 } & \text {-Rluc } \\ \text { TC35 } & \text { TK } & \text {-Rluc } \\ \text { TC33 } & \text { CMV } & \text {-Rluc } \\ \text { TC48 } & \text { CMV } & \text {-EYFPnuc }\end{array}$

The plasmids were mixed at the following ratio: SV40:TK:CMV $=10: 2: 1$ to compensate for the different potency of the promoters.

The Renilla mix is prepared with the following recipe

$\begin{array}{lll}\text { pSV40_Renilla } & 100 \mu \mathrm{g} & \\ \text { pTK_Renilla } & 20 \mu \mathrm{g} & \\ \text { pCMV_Renilla } & 10 \mu \mathrm{g} & \\ \text { pEYFPnuc } & 13 \mu \mathrm{g} & \text { in } 1300 \mu \mathrm{l}\end{array}$

Final concentration of all $110 \mathrm{ng} / \mu \mathrm{l}$

(100ng Renilla plasmids and 10ng EYFPnuc).

Dependent on the assay the Renilla mix has to be modified to meet the requirements or to prevent usage of promoters which react to the assay. The TK-promoter seems to be promoter of choice for NRG1-ERBB4 assays. The fluorescent protein can be exchanged with other colours e.g.

pEYFP, pEYFPnuc, pECFP Cl, DSRed, pCherry

20ng of the Renilla mix was cotransfected per well along with any firefly reporter gene assay, if not indicated otherwise. For standard luciferase reporter gene assays six technical replicates were analysed to enhance statistical reliability. Transfection efficiency was checked via fluorescent protein expression. Bioluminescence measurement was performed with the help of the Microplate reader Mitras LB940 or Microlumat Plus 96V (Berthold Technologies) and the associated software MicroWin 2000 using the following protocol: 
1) Injection of $40-75 \mu$ firefly luciferase substrate, $2-60 \mathrm{sec}$ reaction time, 1$10 \mathrm{sec}$ measurement of light signals

2) Injection of 40-75 $\mu \mathrm{l}$ Renilla luciferase substrate, 2-60 sec reaction time, 1$10 \mathrm{sec}$ measurement of light signals

The firefly and the Renilla luciferases display high substrate specificity and do not cross-react. Moreover, firefly luciferase activity is blocked by the $\mathrm{pH}$ conditions of the Renilla substrate.

\subsubsection{Statistical analysis}

Data were exported from MicroWin 2000 and analyzed with Excel or GraphPadPrism. For normalisation, the firefly-value was divided by the corresponding Renilla-value to get the relative luciferase unit (RLU). Mean, standard deviation (SD) and standard error of the mean (SEM) of the six technical replicates were calculated.

\subsection{Screening of high throughput assays 96 well formats}

\subsubsection{Automated PLL coating}

The Hamilton Labstar robot was programed to coat 96 well plates. The plates were opened than $30 \mathrm{ul}$ of $\mathrm{PLL} / \mathrm{H}_{2} \mathrm{O}$ were pipetted. The plates were incubated for 20 min., than washed with 2 times $50 \mathrm{ul} \mathrm{H}_{2} \mathrm{O}$. The dried plates were sterilised by one hour UV-light exposition.

\subsubsection{In solution transfection of PC12 cells}

To transfect huge amounts of equal cells with equal plasmids we used the following protocol per one 96well plate. 4,000,000 cells were harvested and diluted in $5 \mathrm{ml}$ phenol red free DMEM. The following plasmids were diluted in 2,5ml Optimem and mixed.

$\begin{array}{ll}2 \mu \mathrm{g} & \text { ERBB4-NTEV-tevS-GV_2HA } \\ 2 \mu \mathrm{g} & \text { PIK3R1-CTEV_2HA } \\ 2 \mu \mathrm{g} & \text { UAS firefly luciferase } \\ 2 \mu \mathrm{g} & \text { TK-Renilla luciferase } \\ 0,5 \mu \mathrm{g} & \text { pECFP Cl }\end{array}$

20ul of Lipofectamin2000 were diluted in $2.5 \mathrm{ml}$ Optimem and mixed.

Both Optimem aliquots were mixed and incubated for 20min. Afterward the $5 \mathrm{ml}$ Optimem and the cells in DMEM were carefully mixed, and incubated at $37^{\circ} \mathrm{C}$ at $5 \% \mathrm{CO}_{2}$ for $2 \mathrm{~h}$.

\subsubsection{Plating of cells}

$10 \mathrm{ml}$ solution, containing $4,000,000$ in solution transfected cells, per 96well 
plate, were stored in the bubble paddle reservoir of the Hamilton Cellstar robot. 100ul per well were pipetted using the 96er pipet head. The homogeneity of the solution over time is sustained by mild paddeling of the reservoir. Keep in mind that the reservoir has a "death volume" of $50 \mathrm{ml} / 5$ plates. The finished plates were stored in the cytomat automated cell culture (34 plates maximum).

\subsubsection{Handling of stable Nrg1-type-I expressing cells}

1,000,000 Nrg1-type-I expressing PC12 cells per plate were harvested and diluted in $10 \mathrm{ml}$ phenol red free DMEM. The cells were placed in the Cellstar robot in the bubble paddle reservoir (see above). 10,000 cells/well were plated in each well. Note, stable cell lines grow slower than normal PC12 cells, probably due to Hygromycin. Start in time with growing the cells.

\subsubsection{Dispension of drugs}

The drugs of the NCC library were diluted in DMSO to an f.c. of $1 \mathrm{mM}$. 80 drugs were used per 96well-plate using the collums 2-11 (blue). 1A-D is further stimulated with $10 \mathrm{mg} / \mathrm{ml}$ EGFld (yellow). 12A-D is inhibited with $100 \mathrm{nM}$

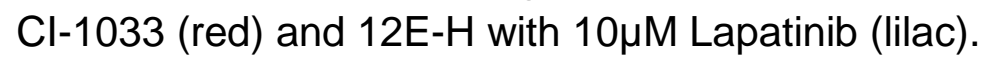

$24 \mathrm{~h}$ after in solution transfection the plates were processed with the Cellstar robot. They were driven out of the incubator, opened, and stimulated with $2 u$ l of Drug/DMSO. 30min. after the drug stimulation, the cells were stimulated with 100ul DMEM with 10,000 Nrg1-typel expressing cells per well, automatically pipetted with the 96 well head from the bubble paddle reservoir. Afterwards the cells were automatically incubated for another $24 \mathrm{~h}$.

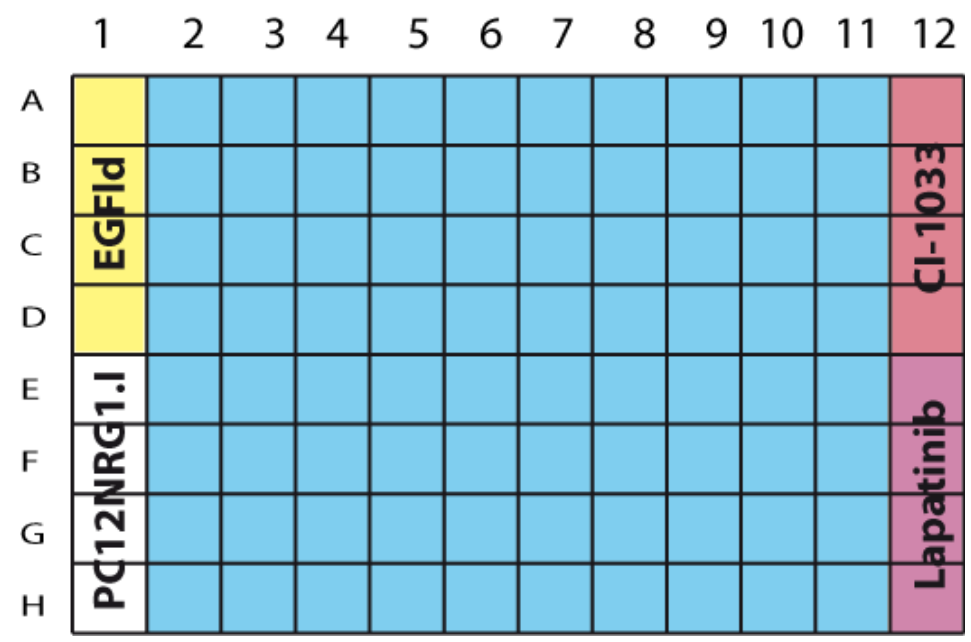

Figure 19: Layout of a 96 well-plate for HTP screen 


\subsubsection{Automated dual luciferase assay}

Plates were emptied of medium. The cells were lysed by application of $35 \mathrm{ul}$ of passive lysis buffer and high speed shaked for $10 \mathrm{~min}$. The plates were measured in the LB plate reader. 30ul of firefly substrate were pipetted in each well, the plate was shaken for ten sec. and than firefly luciferase activity was measured. Than $30 \mu \mathrm{l}$ Renilla substrate were pipeted in each well, the plate was shaken for ten sec. and than Renilla luciferase activity was measured. The data was automaticly exported as text file and analysed using the following TinR script.

\subsubsection{Analysis of results with TinR}

The data was analysed with TinR using the following script:

library("cellHTS2")

\# Script to analyse Luci Screens with cellHTS2

\# part 1 creates all folders and fiules wich are neccesary to run the cellHTS2 package

\# part2 runs cellHTS2

\# part1

\# Please specify Replicate number and number of substance plates subPlates=as.numeric(winDialogString("How many Substance Plates were used", "4")) reps=as.numeric(winDialogString("How many replicates", "3"))

experimentName <- studyName <- winDialogString("Name of the study?", "screen1")

\#create new folder with studyName

datadir<-paste("Z:/User/Systasy project NEW/Operative Business/ERBB4 PI3Kp85a split TEV

Compound screen/Analysis with R/",studyName,sep="')

dir.create(datadir)

dir(datadir)

setwd(datadir)

\#create SUBFOLDERS

dir.create(paste(datadir,"/logRatio",sep="'))

dir.create(paste(datadir,"/rawdata",sep="'))

\# choose all rawdatafiles and copy them to Study folder

csa<-choose.files(default = "Z:/User/Systasy project NEW/Operative Business/ERBB4 PI3Kp85a split

TEV Compound screen/Analysis with R", caption = "Select the LUCI rawdata files",multi = TRUE)

file.copy(csa,paste(datadir,"/rawdata",sep="'))

allfiles <- $\operatorname{dir}($ paste(datadir,"\|rawdatal|",sep="')) ～\# place all raw data files into a RawData subfolder f2r<- allfiles[grep("TXT",allfiles)]

\# go through all rawdata files and extract relevant data and create new files

for(fi in $\mathrm{f} 2 \mathrm{r})\{$

te<-read.table(paste("rawdata/",fi,sep="'),skip=9)

$\mathrm{ff}<-$ te[1:96,]

ffname=paste(strsplit(fi,".TXT")[[1]],"_FF",sep="")

$\mathrm{ff}<-$ cbind (rep(ffname,times=96),ff)

rn<-te[101:196,]

rnname=paste(strsplit(fi,".TXT")[[1]],"_RN",sep="')

rn<-cbind(rep(rnname,times=96),rn)

write.table(ff,paste(ffname,".TXT",sep="'),quote=F,row.names=F,col.names=F,sep="\t")

write.table(rn,paste(rnname,".TXT",sep="'),quote=F,row.names=F,col.names=F,sep="\t") \} 


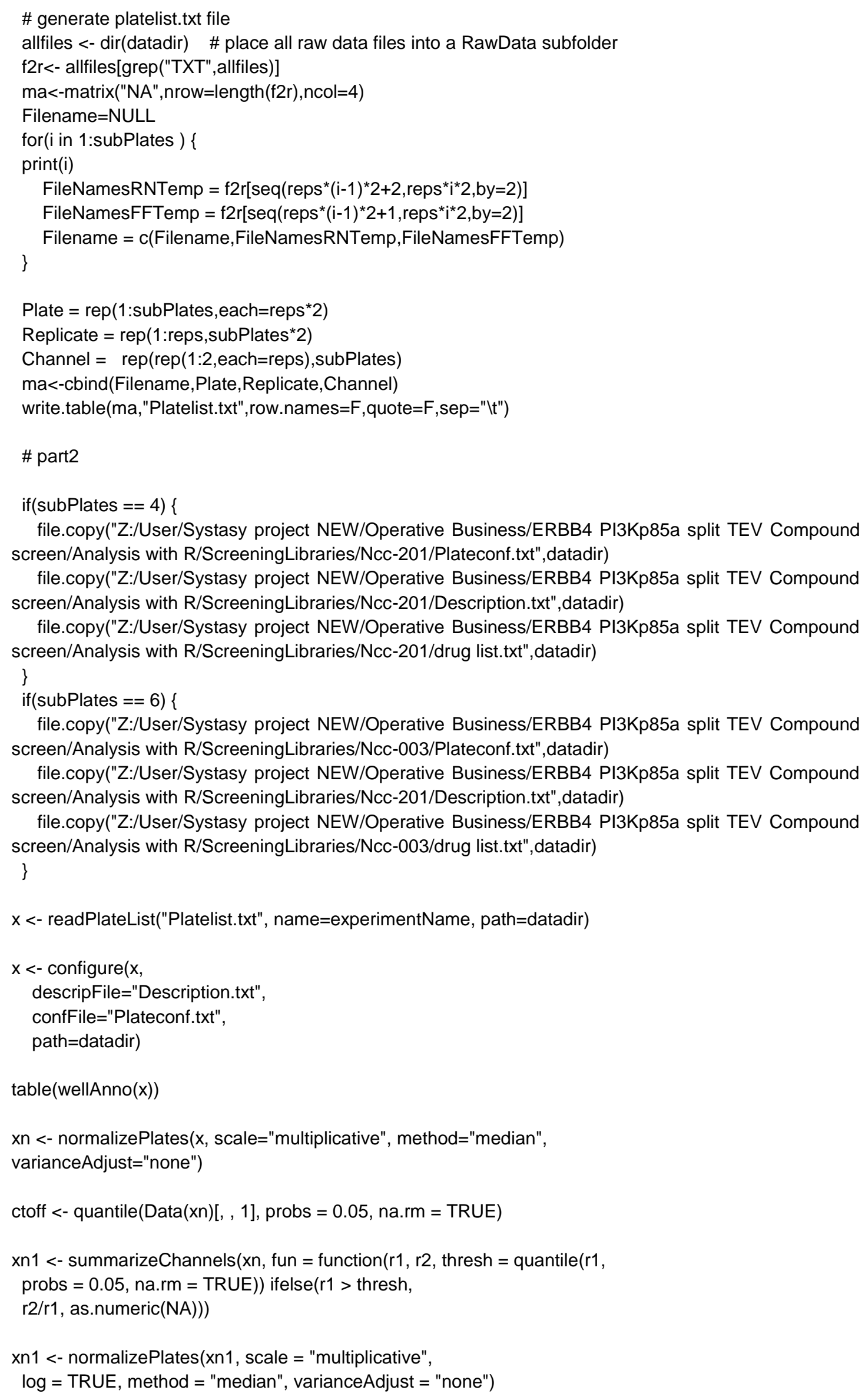




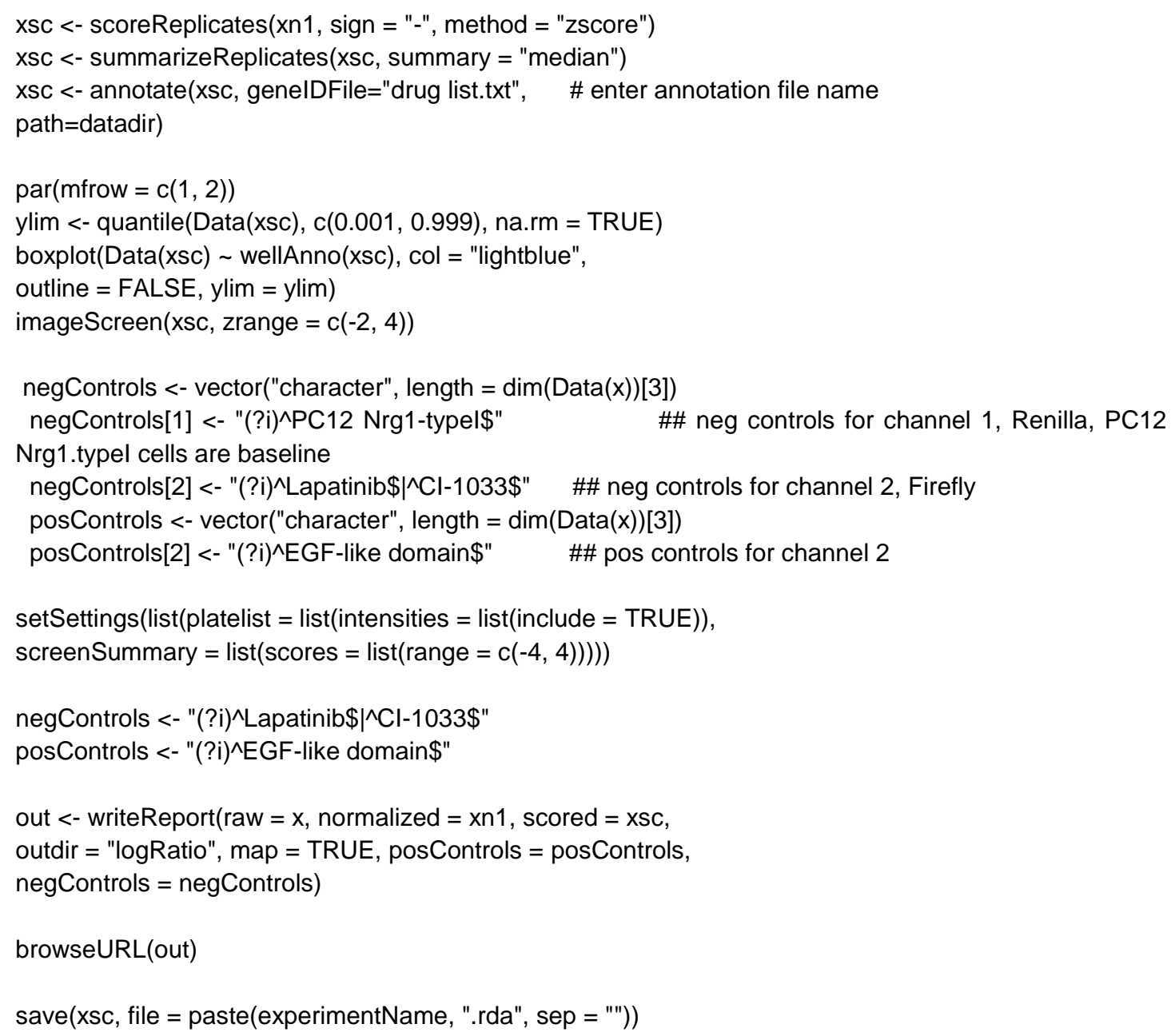




\subsubsection{Preparation of dose response dilutions for 96 well format.}

To prepare a master plate start by solving the drug in DMSO. Prepare 150ul of $10 \mathrm{mM}$ stock solution. Prepare 2 stripes of a 96 well plastic plate with following DMSO amounts.

Pipette 100ul stock solution in 1a, pipette 34 ul stock solution in $1 \mathrm{~b}$, mix by pipetting 10times up and down. Transfer $10 \mathrm{ul}$ from 1a. $\rightarrow 2 \mathrm{a}$., mix, transfer 10ul from $2 \mathrm{~b} . \rightarrow 2 \mathrm{c}$., repeat top down pipetting for all wells except $7 \mathrm{~b}$. that remains pure DMSO. 8a. and b. remains empty for assay stimulation controls.

Dose response curves from f.c. $100 \mu \mathrm{M}$ to $0.0001 \mu \mathrm{M}$ can now be generated by pipetting $2 \mathrm{ul}$ to wells containing final $200 \mathrm{ul}$ co-culture or cell culture buffer. Note pipetting of small amounts (2ul) with 8 channel pipets is inaccurate 12ul are needed for 6 wells. Plan with $150-200 \%$ as master mix. $90 \mu$ will only be secure enough for $5^{\star} 96$ well plates.

\subsection{Biochemical methods}

\subsubsection{Western Blot}

Western blotting is a classical method for immunological detection of proteins in biological samples (Towbin et al., 1992).

\subsubsection{Sodium dodecyl sulphate polyacrylamid gel electrophoresis (SDS-PAGE)}

Unlike DNA, proteins may be positively or negatively charged or neutral and do not have a linear relationship between weight and charge due to differences in amino acid composition. To enable directed migration of proteins in the electric field SDS is used to produce a negative charge. After heat denaturation at $70^{\circ} \mathrm{C}$ the polypeptide chains unfolds to expose the hydrophobic regions which can non-covalently bind SDS hydrophobic tails at the stochiometry of 1 SDS molecule per 2 amino acids. Additional treatment with dithiotreitol (DTT) reduces all intra- and intermolecular disulfide bonds.

Protein lysates were mixed with $4 x$ LDS sample buffer and 10\% DTT (f.c. $0.1 \mathrm{M}$ ) and incubated at $70^{\circ} \mathrm{C}$ for $10 \mathrm{~min}$. 2- $5 \mu \mathrm{g}$ were used for western blotting. For SDS-PAGE NuPAGE Novex Bis- Tris Gels (Invirtogen) were used with Xcell SureLock NuPAGE buffer chambers (Invitrogen) according to the manufacturer's protocol. The electrophoresis was performed at $200 \mathrm{~V}$ for 45 $60 \mathrm{~min}$. 


\subsubsection{Transfer of proteins on membranes}

The transfer of proteins from the gel to a PVDF membrane was performed with the Xcell SureLock Western Blot System (Invitrogen) according to the manufacturer's instructions. Prior to transfer PVDF membranes were activated for $3 \mathrm{~min}$ in $100 \%$ methanol and afterwards rinsed in transfer buffer. The transfer was done at $30 \mathrm{~V}$ for $2 \mathrm{~h}$ while the chamber was placed in ice water.

\subsubsection{Detection}

When the transfer was completed the membrane was incubated in blocking buffer (5\% non fat milk powder in TBS-T for normal antibodies or 5\% BSA in TBS-T for $\alpha$-phospho antibodies) for at least 30min at RT. The primary antibody was applied in the appropriate dilution in blocking buffer at $4^{\circ} \mathrm{C}$ overnight. The membrane was rinsed twice with TBST and than washed with TBST three times for $10 \mathrm{~min}$ and was subsequently incubated with the appropriate horse-radish peroxidase (HRP)-conjugated secondary antibody for $1 \mathrm{~h}$ at RT. After two times rinsing and three wash steps for $10 \mathrm{~min}$ in TBS-T the membrane was treated with enhanced chemiluminescence (ECL) detection solution for $1 \mathrm{~min}$ according to the manufacturer's recommendations and enclosed in plastic foil. In a dark chamber an ECL-hyperfilm was exposed to the membrane and developed with KODAK-O-MAT. For reprobing the membrane was incubated with $0,5 \mathrm{M} \mathrm{NaOH}$ for 3 min at RT or Restore WB stripping buffer according to the manual. The membrane was then washed in TBS-T and blocked again as described above. All incubation steps were performed under constant moderate shaking or rolling.

\subsection{The MK801 mouse model}

NMDA antagonists are tools to produce SZ-like symptoms in animal models and human subjects (Eyjolfsson et al., 2006). This is based on the first findings (Luby et al., 1959) that Phencyclidine induces a psychotic state with positive and negative symptoms in healthy humans (Snyder, 1980). The underlying mechanism of glutamate receptor blockage was discovered in 1982 (Lodge and Anis, 1982). The dysfunction of the combined dopaminergic and glutamatergic transmitter system in the pathogenesis of SZ has been broadly discussed (Javitt and Zukin, 1991; Olney and Farber, 1995; Flores and Coyle, 2003; Carlsson et al., 1999). The disturbance of glutamatergic neurotransmission, has been reported in patients with distinct negative symptoms and cognitive deficits (Harrison and Weinberger, 2005; Goff and Coyle, 2001; Tsai and Coyle, 2002). All known SZ susceptibility genes act on glutamatergic synaptic transmission (Harrison and Weinberger, 2005), and 
reduced GABA synthesis has also been described in SZ patients (Lewis et al., 2005).

Therefore, MK801, the most potent non-competitive NMDA receptor complex antagonist, is used to model SZ symptoms in animal models. MK801 binds to a site in the ion channel and blocks cat ion flow. Therefore, the receptor acquires the open confirmation that is dependent on the agonist MK801.

The drug produces locomotor hyperactivity in rodents that may correspond to positive symptoms of SZ (Nilsson et al., 2004), social withdrawal that is a core phenotype of negative symptoms (Sams-Dodd et al., 1997), pre-pulse inhibition deficits, and maybe cognitive deficits (Arnt, 1998).

MK801 is reported to trigger auditory hallucinations (Allen and Young, 1978).

In addition, MK801-elicited effects were studied on hippocampal and prefrontal GABAergic interneurons in rodent animal models (Braun et al., 2007).
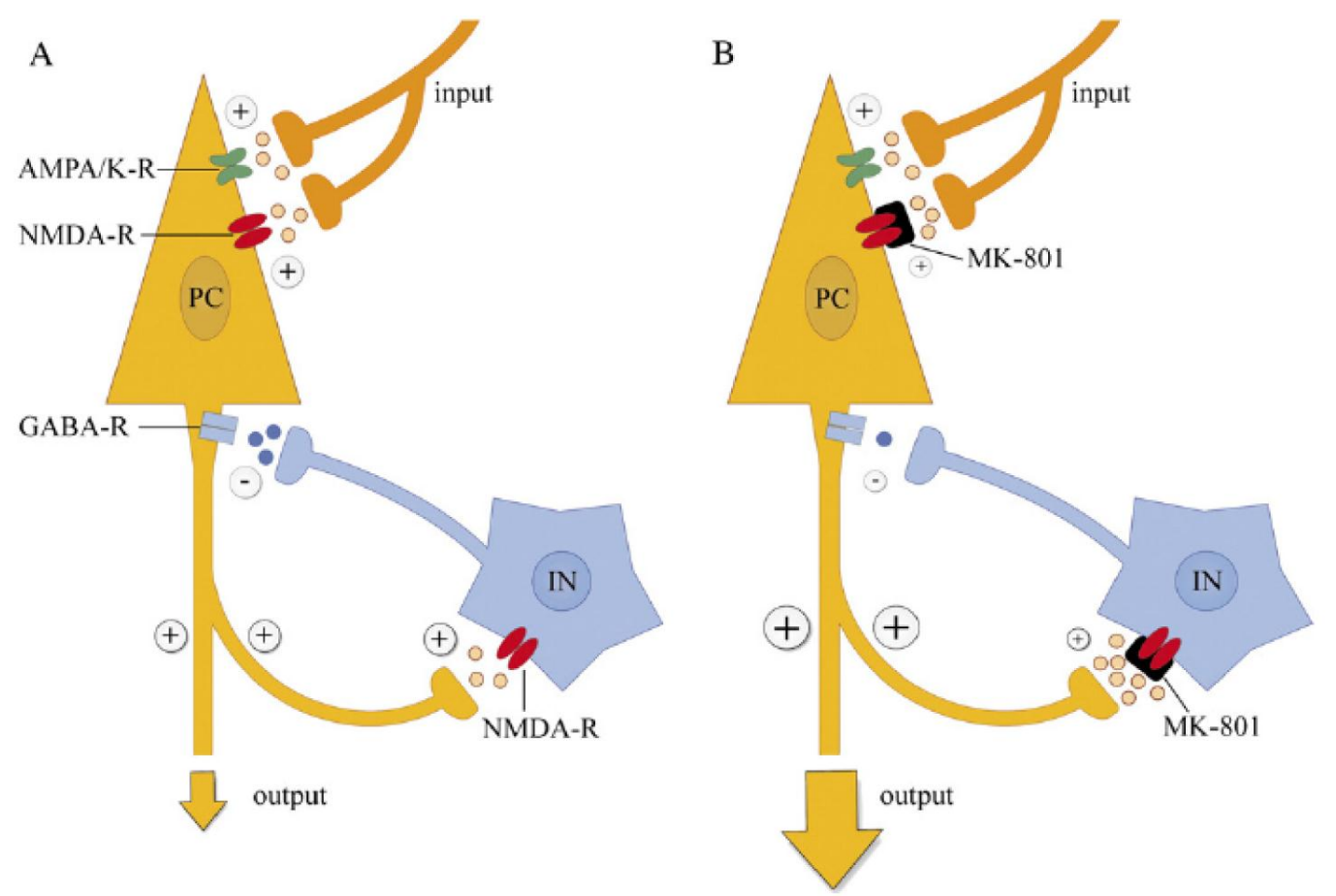

Figure 20: Model of the local neuronal circuit dis-inhibition in response to MK801 in the cortex.

A) An inhibitory GABAergic interneuron (IN, i.e. Chandelier cell or Basket cell) receives input from the excitatory pyramidal cell $(\mathrm{PC})$ thereby exerting an inhibitory control by recurrent projections to the pyramidal cell. B) In presence of the NMDA receptor antagonist MK801, this local feedback inhibition becomes disrupted, whereas the excitatory input is sustained via non-NMDA (AMPA/kainate) receptors, which do not respond to MK801. Due to this imbalance the total excitatory output is predicted to be enhanced. Image taken from (Braun et al., 2007). 


\subsection{Drug tests in mice}

\subsubsection{Drugs and treatments}

Spironolactone (Sigma-Aldrich) was dissolved in $0.9 \% \mathrm{NaCl}$ with $1 \% \mathrm{DMSO}$ and $0.002 \%$ Tween ${ }^{\circledR} 20$. MK801 (Sigma-Aldrich) was dissolved in $0.9 \% \mathrm{NaCl}$ with $1 \%$ DMSO. Drugs were stored in DMSO at $-20^{\circ} \mathrm{C}$ and diluted freshly before experiments. Spironolactone $(20 \mathrm{mg} / \mathrm{kg}), \mathrm{MK} 801(0.5 \mathrm{mg} / \mathrm{kg})$, and vehicle $(0.9 \% \mathrm{NaCl}$ with $1 \% \mathrm{DMSO})$ were injected i.p. in volumes of $10 \mathrm{ml} / \mathrm{kg}$.

\subsubsection{Mice}

C57Bl6/N male mice were purchased from Charles River (Sulzfeld, Germany) at the age of three weeks. After arrival, animals were housed in standard plastic cages with food and water ad libitum in the colony room, with a 12 hours light-dark cycle (lights switched on at 8:00 a.m.). One week prior experiments, mice were placed in single cages and habituated to experimental rooms. Experiments were conducted with nine weeks old animals. Treatment groups were analysed at balanced time points during the light phase to exclude an interference of circadian rhythm on drug actions.

\subsubsection{Behavioral studies}

To assess the effects of Spironolactone on MK801-induced hyperactivity mice were injected with corresponding drugs according to the protocol depicted in (Fig. Figure 55 A) Animals were divided randomly in one of the following four treatment groups: Group 1 was injected twice with vehicle ([veh+veh], $n=13$ ), group 2 was administered with Spironolactone and vehicle ([Spiro+veh], $\mathrm{n}=14$ ), group 3 treated with vehicle and MK801 ([veh + MK801], $n=12$ ), and group 4 injected with Spironolactone and MK801 ([Spiro+MK801], $n=14$ ). $100 \mathrm{~min}$ after the first injection (vehicle or Spironolactone) each animal underwent the open field test. The open field test was conducted in a Plexiglas arena (45 × $45 \times 55 \mathrm{~cm}$ high). Each mouse was placed individually in an open field box and was allowed to explore freely the test arena for 20 min to determine baseline activity. After 20min, the experiment was stopped for $4 \mathrm{~min}$ to administer the second injection (vehicle or MK801 according to the protocol in (Figure $55 \mathrm{~A}$ ) Subsequently, animals were placed back to the open field box and left undisturbed for $120 \mathrm{~min}$. The distance travelled, the time which each mouse spent active, and the time spent in the centre of the arena (defined as $70 \%$ of central area) were analysed as an indicator of anxiety. The numbers of rearings were taken as a sign of exploratory drive. All movements were monitored by infrared sensors and analysed by the ActiMot software (TSE Systems, Bad Homburg, Germany). 


\section{Results}

\subsection{Design of a screening platform for Nrg1-ERBB4 signalling}

A cell-based assay that can be used for HTP screening approaches has to meet specific criteria, such as scalabilty and, robustness. Moreover, it has to provide a simple but quantifiable readout.

The screening assay described here is mimicking proximal aspects of the Nrg1-ERBB4 signalling using a co-culture assay of two different cell populations (Figure 25). The first population represents PC12 cells expressing constitutively active mouse-derived Nrg1-typel- $\beta 1$ a (Figure 26, Figure 27). The second population represents also PC12 cells but expressing human ERBB4 fused to the N-terminal part of the TEV protease (NTEV) that is linked via a TEV protease cleavage site (tevS, sequence: ENLYFQ'G) to the artificial transcription factor Gal4-VP16 (GV). As ERBB4 is a type I transmembrane receptor, GV is anchored to the membrane as long as no functional TEV protease is present. Activated ERBB4, i.e. Nrg1 ligand-bound and dimerised ERBB4, is auto-phosphorylated at several specific Tyr residues in the Cterminal region that act as binding sites for phospho-adapter molecules, such as the regulatory subunit of the PIK3, PIK3R1, that binds to phosphorylated Tyr residues within YXXM motifs. Therefore, PIK3R1 fused to the C-terminal part of the TEV protease (CTEV), is also expressed in the second cell population. In this assay, activated ERBB4 binds to PIK3R1 initiating the formation of TEV protease activity as both TEV protease fragments get into close proximity, which allows the refolding of the inactive fragments into a functional TEV protease. The protease can cleave at the tevS to release the artificial transcription factor GV that in turn translocates to the nucleus. Here, GV induces the transcription of a firefly luciferase reporter gene that is under the control of upstream activating sequences (UAS-Fluc). UAS-Fluc is the third component that has to be introduced into the second cell population to complete the assay. Using a firefly luciferase as readout allows for a linear amplification of an occurred ERBB4/PIK3R1 interaction event. In addition, the luciferase assay is an easy-to-use readout, stable, and very sensitive assay (Figure 22, Figure 28).

\subsubsection{Normalisation}

For normalisation purposes, the assay system is co-transfected with a Renilla luciferase vector. The Renilla luciferase is constitutively expressed under the control of the Thymidine kinase (TK) promoter. It is assumed that the transfection efficiency can be compared across all wells using the enzymatic activity of the Renilla luciferase. Choosing the correct promoter for the Renilla 
luciferase is vital, as some promoters like the CMV or SV40 promoter seem to respond to growth factors, such as the EGFld and other introduced effects like shRNAs (C. Hammer personal communication). In addition, the normalisation vector in the screen is used to detect toxic substances by means of absolute Renilla values.

\subsubsection{Concentrations of plasmids}

All plasmids in the assay are regularly used in the concentration of 20ng/well. Titration assays have shown that this concentration is sufficient to yield optimal induction ratios for the Nrg1-induced ERBB4/PIK3R1 assay, which is also in agreement with a previous report using the split TEV technique for ERBB4 assays (Wehr et al, 2008).

\subsubsection{Cell numbers per well}

Two different cell concentrations have to be considered. First, cell numbers of population 1 have to be high enough to provide a detectable and stable signal, but adjusted to reasonable levels to prevent overcrowding as cells still divide within the $48 \mathrm{~h}$ duration of the assay. 40,000 PC12 cells/96-well were determined to be optimal. Second, the Nrg1-typel-expressing cells have to be adjusted. A number of 5,000-10,000 cells/96-well resulted in a stable activation of the assay that allowed both further activation or inhibition.

\subsubsection{Cell types tested for the assay}

We tested PC12, HEK293, and CHO cells for usability in the screen. PC12 cells performed best in transient assays. HEK293 cells gave unstable results in transient assays and, unfortunately, a triple transgenic HEK293-derived cell line stopped working reliably. $\mathrm{CHO}$ cells gave no stable results at all.

\subsubsection{Transfection methods}

We tested FuGeneHD, Lipofectamine LTX, and Lipofectamine 2000. For PC12 cells, Lipofectamine 2000 was best suitable, as it could well be used for both adherent and in-solution transfections. For screening purposes, insolution transfection was used to increase homogeneity of transfected cells in the wells. A special steering device named "bubble paddle reservoir" available within the Hamilton Microlabstar Plus robot was used to further increase homogeneity of the cells.

\subsubsection{Workflow of the NRG1-ERBB4 assay}

Critical to HTP-screening are the time points applied in a workflow of a given assay. When using in-solution transfection, two time points are critical. First, the components of the assay have to be expressed and correctly localised to 
the membrane, a process that occurs during the expression phase of the transiently transfected cells after plating. Second, a good time window has to be chosen for the application of the drugs and concomitant addition of the Nrg1-type1-expressing cells. We tested various setups with six hours differences between each time point and found a reasonable measurement window between $12 \mathrm{~h}$ expression/12h stimulation and $24 \mathrm{~h}$ expression/36h stimulation. After a total assay duration of $72 \mathrm{~h}$, the plates were overgrown and the cells started to die. For screening purposes, working hours also have to be considered. Therefore, a $24 \mathrm{~h}$ expression/24 stimulation workflow was chosen.

\subsubsection{Stability of the luciferase signals}

To provide a robust readout the luciferase signal has to be stable over the time needed for the measurement. For HTP screening, the processing of the assay plates should be as fast as possible. Therefore, cells are lysed using an 8-well dispenser head, allowing to dispense the buffer in minimal time. In addition, the buffer used, the Promega passive lysis buffer, is formulated to provide optimal stability for luciferase enzymes. When the substrates are added to the cell lysates, the luciferase reaction starts immediately. The firefly luciferase signals are relatively stable, with $80 \%$ stability over the first 5 minutes. The Renilla luciferase signals decrease fast, with a reduction of $50 \%$ within two minutes. To start the measurement, luciferase substrates are dispensed across the plate, followed by orbital mixing and the measurement itself for which the detector head moves again across the plate. The minimum time required for a measurement of one plate in one run in our luciferase reader is 100 seconds for dispensing, 10 seconds for mixing, and 100 seconds for reading. Thus, firefly and Renilla luciferase activity is measured in each well 110 seconds after addition of the substrate. As the timing for each well regarding substrate addition and reading is the same the initial dramatic decrease in Renilla luciferase activity should not have an effect on the readings obtained from the screen (Figure 21). 
Results

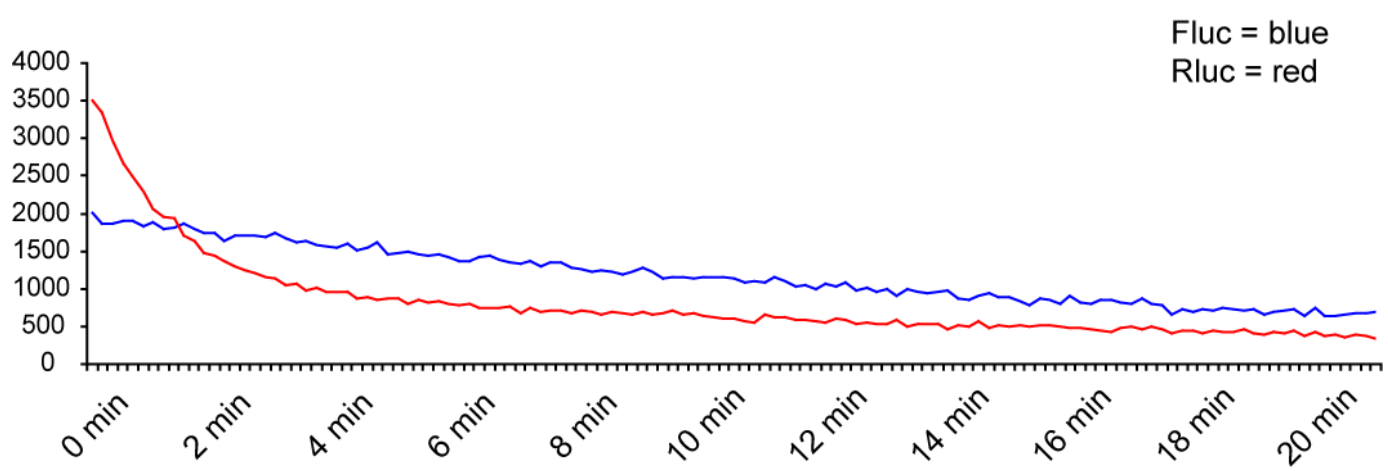

Loss of function Rluc 120 sec. in \%.

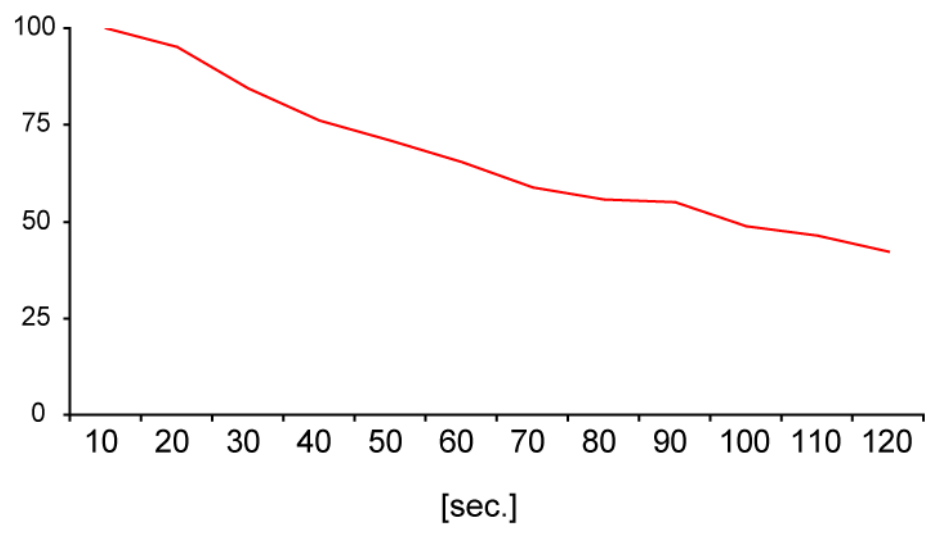

\section{Figure 21: Kinetics of firefly and Renilla luciferase}

Decay of firefly luciferase (Fluc, blue) and Renilla luciferase (Rluc, red) signal over $20 \mathrm{~min}$. The Fluc signal drops regularly over 20 min, then remaining with about $50 \%$ of activity. The Rluc signal initially drops fast, losing about $50 \%$ of its activity within the first 2 min, but remains relatively stable afterwards. 


\subsubsection{Protocol at a glance one 96 well plate}

The experiments lead to the following protocol used in the screening assay:

Transfection Protocol

1. Pipet DNA

2. Mix DNA with $1 / 2$ volume Optimem

3. Vortex

4. Incubate $2-5$ minutes

5. add LF2000 to other $1 / 2$ volume Optimem

6. Vortex

7. Incubate $2-5$ minutes

8. Combine LF2000/Optimem and DNA/Optimem mix

9. Vortex

10. Incubate 30 minutes at RT

11. Add cells (in medium, with same volume as total volume of Optimem)

12. Incubate $2 \mathrm{~h}$ at $37^{\circ} \mathrm{C}$, but no shaking/rocking

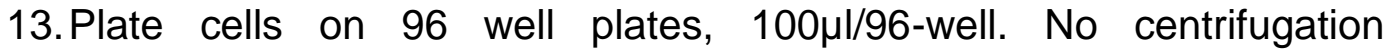
necessary

14. Incubate $24 \mathrm{~h}$ for protein expression. Check expression using a GFPtransfected control plate.

15. Add drugs $(2 \mu \mathrm{l}$ in DMSO, f.c. $10 \mu \mathrm{M})$. Controls: $10 \mu \mathrm{M}$ Lapatinib, 0.1 $\mu \mathrm{M} \mathrm{Cl}-1033$.

16. Add EGF-like domain (f.c. $10 \mathrm{ng} / \mathrm{ml} ; 20 \mathrm{ng} / \mathrm{ml}$ added in $100 \mu \mathrm{l}$ ) or PC12 Nrg1-typel cells (in 100 $\mathrm{\mu l}$ )

17. Incubate further $24 \mathrm{~h}$

18. lyse cells for assay ( $35 \mu \mathrm{l} \mathrm{PLB/96-well)}$

\subsection{Component controls}

\subsubsection{CMV-GV}

The PC12 cells are transfected with UAS-Fluc and GV. The UAS-Fluc can be activated by constitutively active expression of the GV that is under the control of the CMV promoter. In HTP screens, all substances that interfere with Fluc activity have to be eliminated to reduce false positive signals (Figure 23).

\subsubsection{TM-TEV/TM-GV}

The PC12 cells are transfected with TM-TEV, TM-GV, and UAS-Fluc. TEV protease and GV are bound to a membrane anchor termed TM, a transmembrane domain present in the plasmid pHOOK. Both the protease and the GV are localised to the membrane. Any substance that interferes with TEV protease activity can be removed from the screen (Figure 23) 


\subsubsection{Lapatinib}

Lapatinib is an ERBB-specific receptor tyrosine kinase inhibitor. In the screen, it is used as control acting as an inhibitor. $10 \mu \mathrm{M}$ of Lapatinib leads to highly decreased luciferase activity in the screen (Figure 29).

\subsubsection{CI-1033}

$\mathrm{Cl}-1033$ is a non-specific receptor tyrosine kinase inhibitor. It is used in the screen as further control also acting as a highly potent ERBB4 inhibitor. $100 \mathrm{nM}$. Cl-1033 results in even more decreased luciferase activity compared to $10 \mu \mathrm{M}$ Lapatinib (Figure 29).

\subsubsection{EGFld}

The NRG1 EGF-like domain (EGFld) is a potent activator of ERBB3 and ERBB4 activity. In the screen, it is used to further activate the assay, with levels that are above the ones induced by Nrg1-typel-expressing cells. $10 \mathrm{ng} / \mathrm{ml}$ EGFld (ca1.5nM, MW ca. 7.2 kD) are sufficient to activate the assay nearly to maximum levels. EGFld domain represents the functional domain of human NRG1 for ERBB3/4 activation. Therefore, EGFld represents the most effective way to activate ERBB3/4. Substances that are found in the screen and modulate Nrg1-ERBB4 signalling, but fail to modulate EGFld-ERBB4 signalling act as putative modulators of Nrg1 processing (Figure 24). 
Results

A

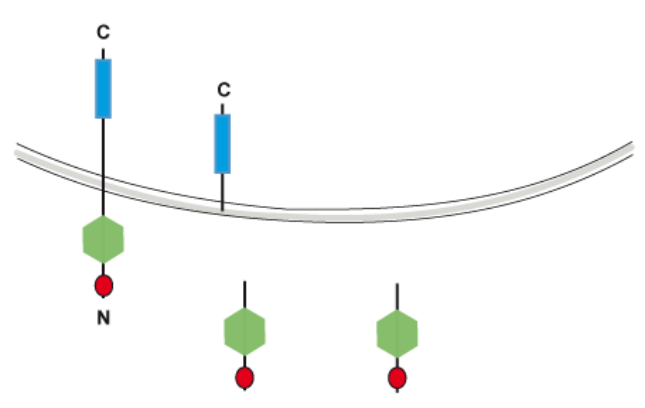

C

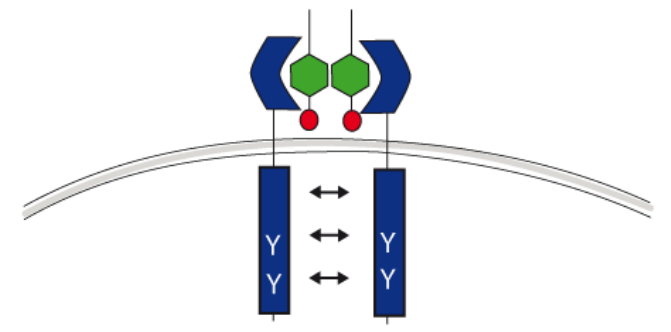

E

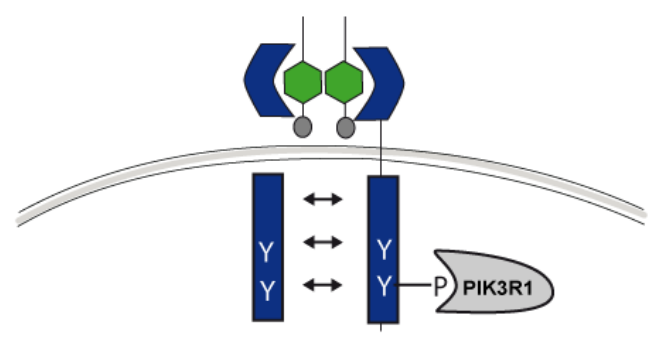

G

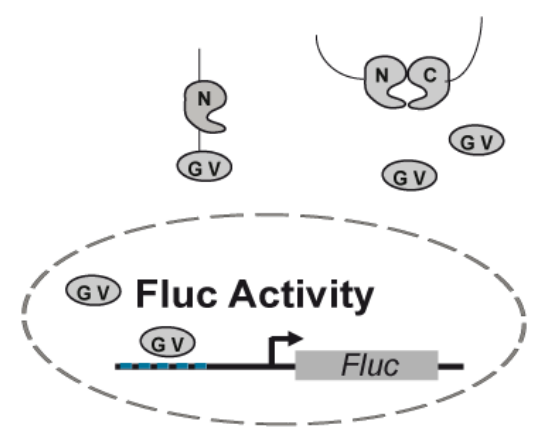

B

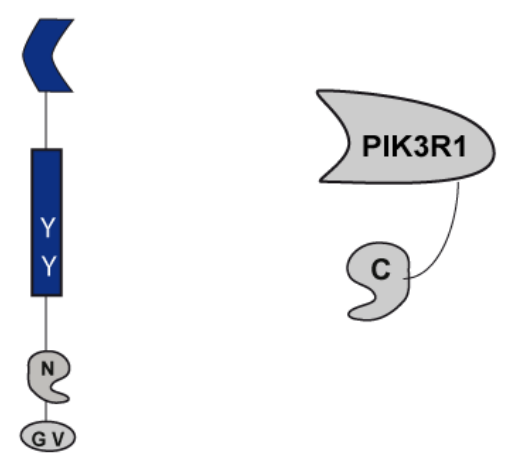

D

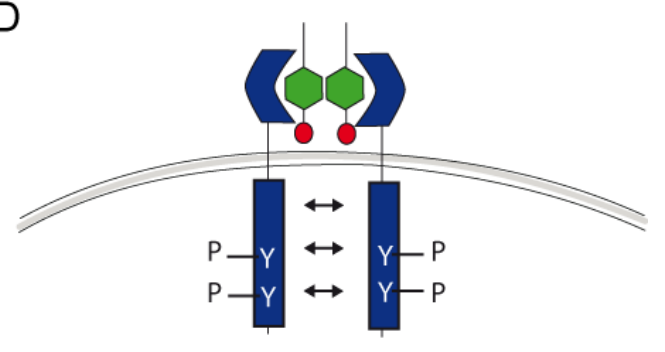

F

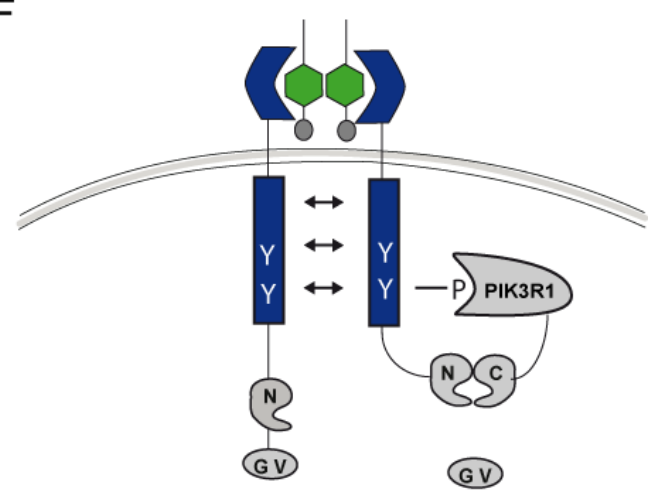

$\mathrm{H}$ 
Figure 22: Schematic representation of the shape and function of the constructs used in the screen

A) Distal of the signalling cascade is a PC12 cell line stable expressing Nrg1-typel $\beta 1$. Nrg1 is localised in the membrane, processed, and then released into the medium. B) The ERBB4 receptor is fused to the N-terminal moiety of the TEV protease. In addition, it is fused to the GalV-VP16 transcription factor via the TEV protease-specific cleavage site ENLYFQ 'G. The alpha regulatory subunit of the PI3-kinase, PIK3R1, is fused to the Cterminal moiety of the TEV protease. C) The ERBB4 receptor homodimerises after binding to Nrg1-typel. D) After forming homodimers, ERBB4 receptor pairs auto-phosphorylate each other. E) The ERBB4 homodimer recruits the PIK3R1 to the PI3-kinase binding site at Tyr 1056.F) The recruitment of PIK3R1 to the ERBB4 homodimer brings the N- and Cterminal fragments of the TEV protease in close proximity. G) Both fragments of the TEV protease fold into the active protease and regain protease activity. The TEV protease targets the TEV-specific cleavage site and releases the Gal4-VP16 transcription factor. The transcription factor shuttles to the nucleus and activates the UAS-Fluc reporter gene. $\mathrm{H})$ Activation of the UAS-Fluc reporter gene results in a higher firefly luciferase activity, which can be measured by the turnover of ATP and d-luciferine producing light. 
A Renilla Luciferase
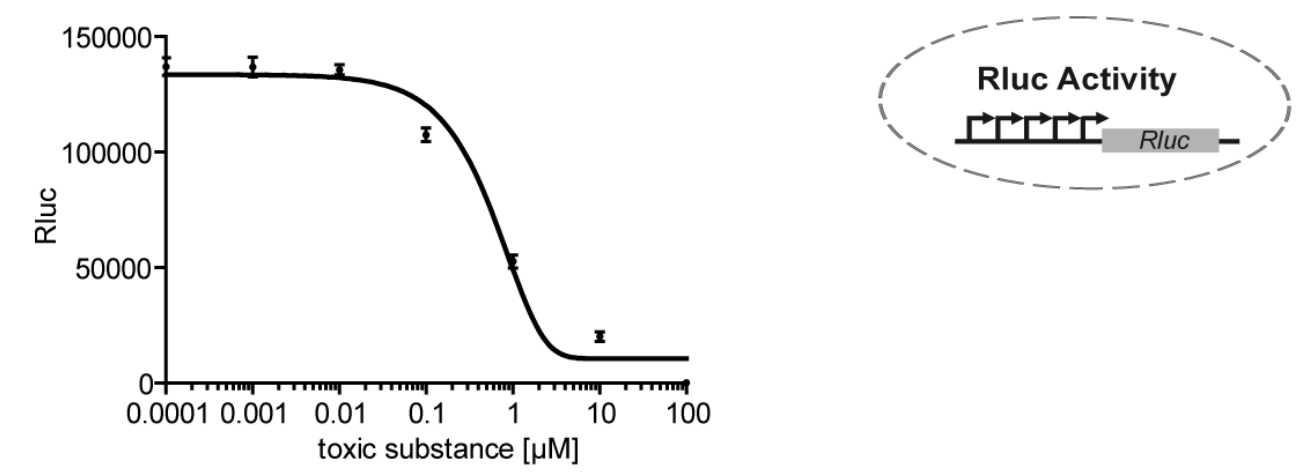

B CMV-GV/G5-FFLuc

$$
z^{\prime}=0,8
$$
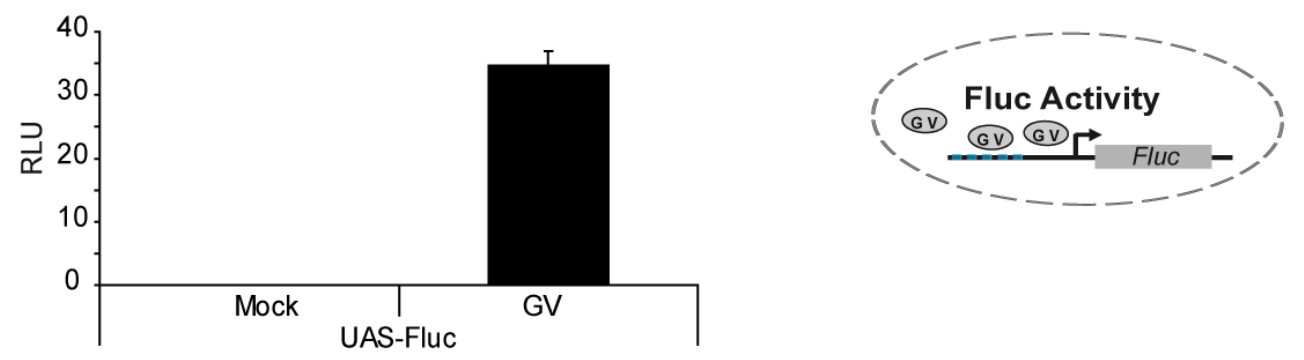

C TM-TEV/TM-GV
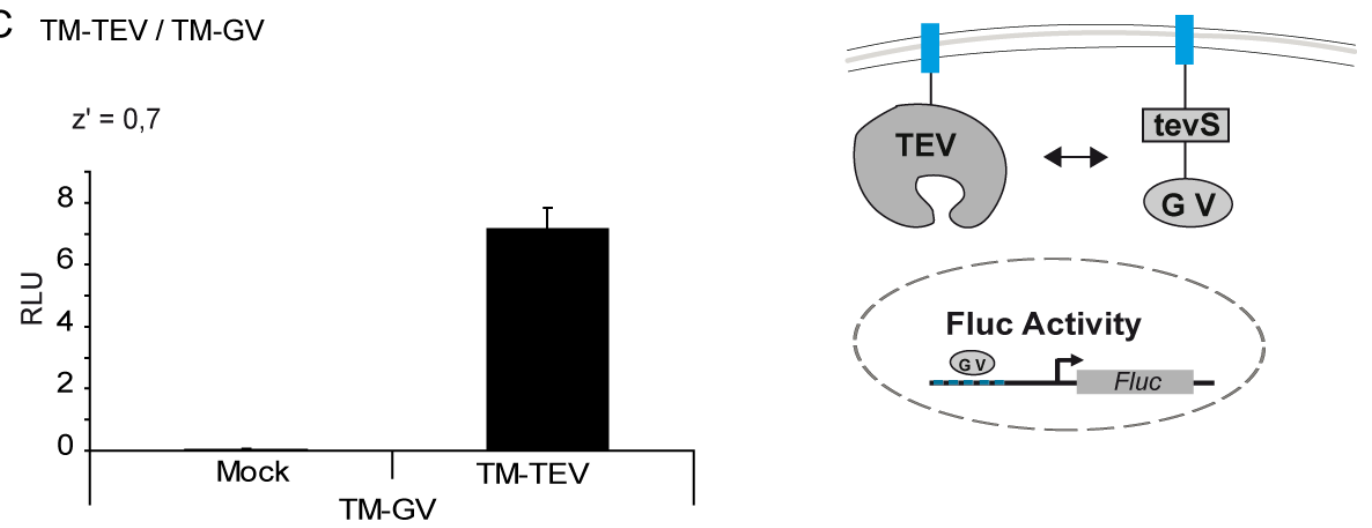

\section{Figure 23: Controls of the screen}

A) Renilla luciferase. Effect of a toxic substance. B) Constitutively active GV-UAS-firefly assay. The Cytomegalovirus promoter (CMV) was placed in front of the Gal4-VP16 (GV) artificial transcription factor. C) TM-TEV/TM-GV.A transmembrane anchor (HOOK) was fused to the TEV protease (TM-TEV) and the TEV protease cleavage site/GV fusion (TMGV). 
Results

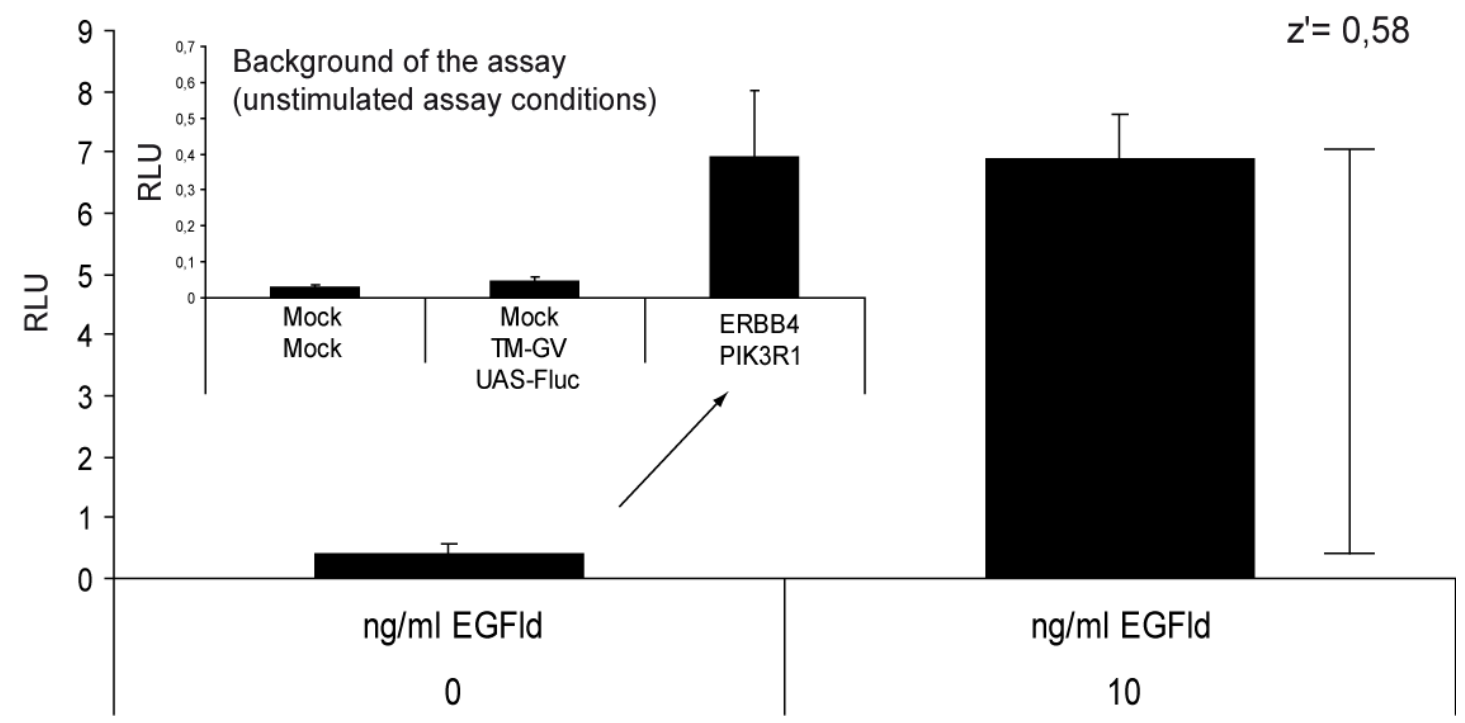

Figure 24: Background of the assay

ERBB4/PIK3R1 split TEV assay in PC12 cells. ERBB4 was fused to NTEV-tevS-GV-2HA. PIK3R1 was fused to CTEV-2HA. ERBB4 dimerises after stimulation with $10 \mathrm{ng} / \mathrm{ml}$ EGFlike domain and recruits PIK3R1. Further controls (inset) show the background of the assay. The background of non-stimulated ERBB4/PIK3R1 is about ten times higher than the background of mock transfected cells or cells transfected with TM-GV/UAS-Fluc. 
A

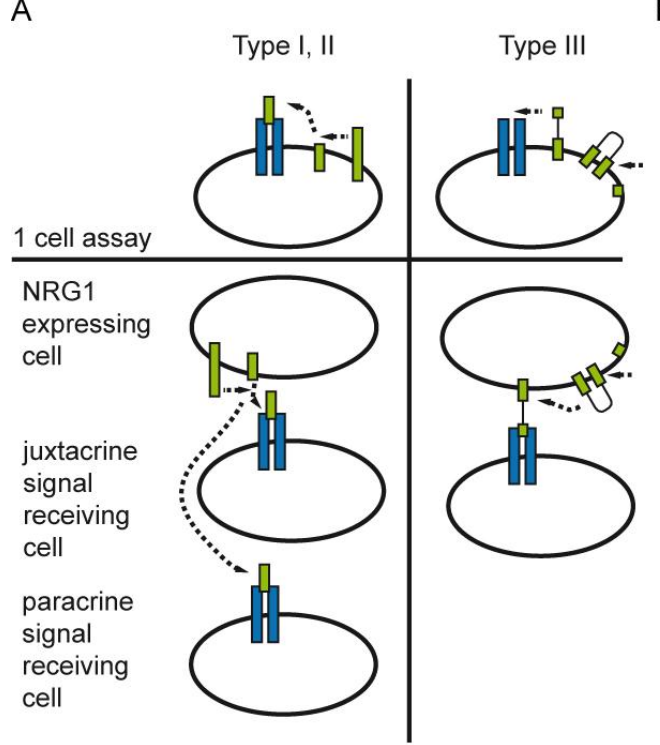

C Brightfield
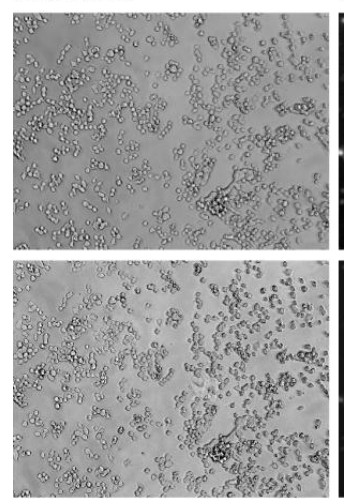

D
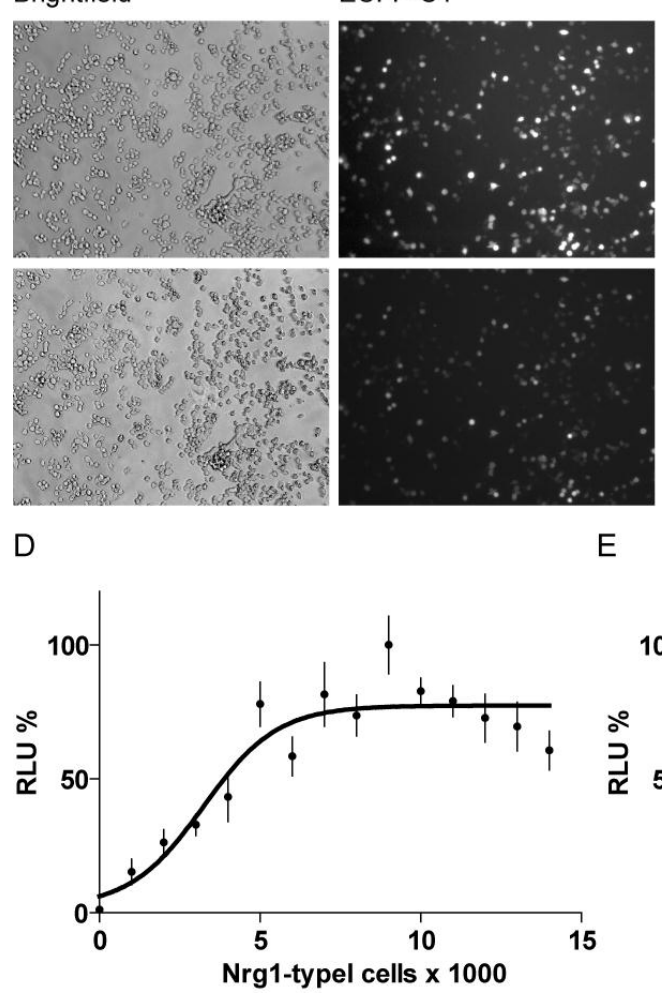

$\mathrm{E}$

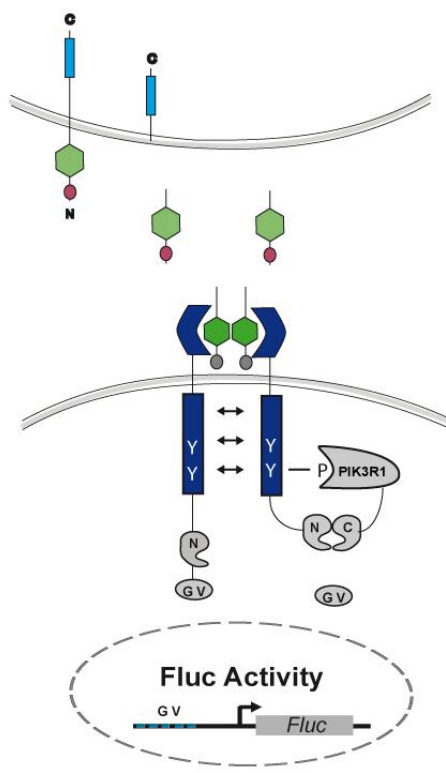

EYFPnuc
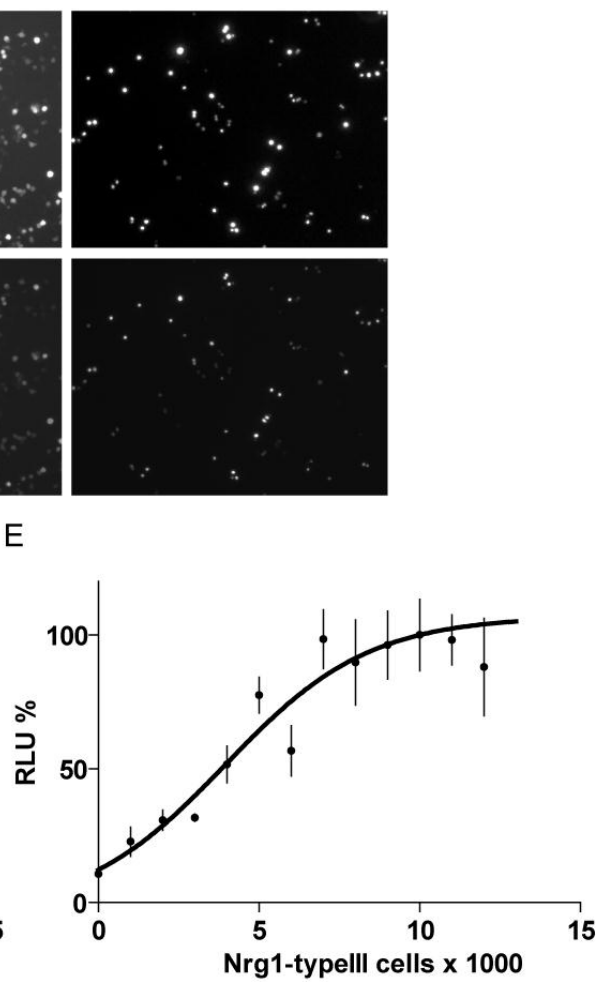

Figure 25: Design of the co-culture assay for the screen

A) Schematic representation of the differences between Nrg1-typel- and Nrg1-typelllmediated signalling in co-culture assays. B) Schematic representation of the co-culture assay. Cell population one expresses Nrg1-typel. The receiving cell, i.e. cell population two, expresses ERBB4-NTEV-tevS-GV-2HA and PIK3R1-CTEV-2HA. After binding to NRG1-typel, ERBB4 homodimerises and recruits PIK3R1. TEV protease activity is reconstituted, cleaving at the tevS to release GV. GV translocation to the nucleus results in increasing Fluc activity.C) Microscopic images of the co-culture assay. 40,000 PC12 ERBB4/PIK3R1 $\square$ cells were transfected with ECFP-C1; 10,000 Nrg1-typel cells were transfected with EYFPnuc. D) Dose response of the Nrg1-ERBB4 assay using increasing numbers of Nrg1-typel-expressing cells. E) Dose response of the Nrg1-ERBB4 assay using increasing numbers of Nrg1-typelll-expressing cells. 
A

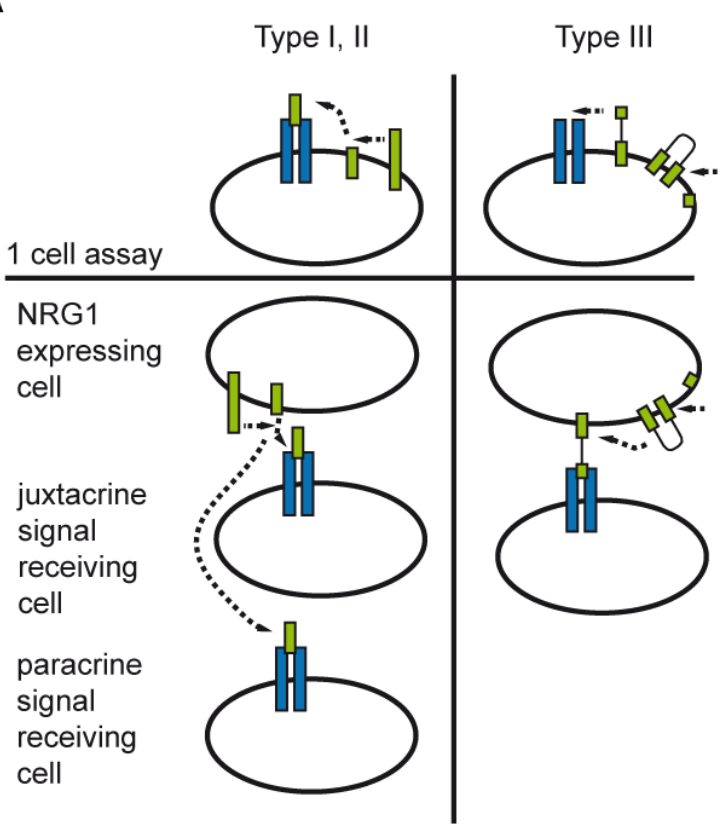

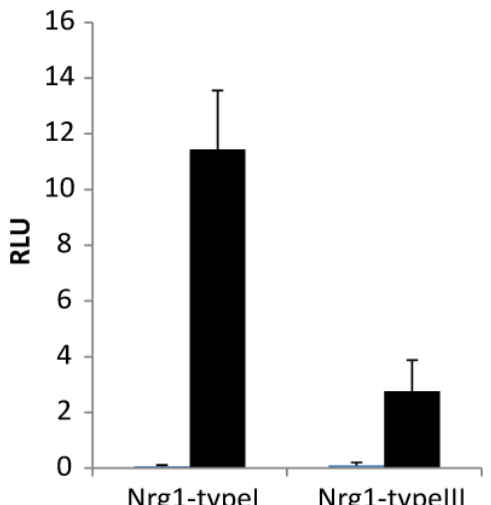

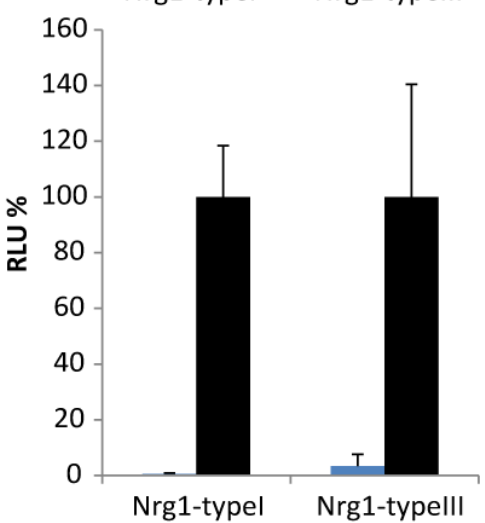

Figure 26: Difference between stimulation with Nrg1-typel and NRG1-type III stable cell lines

ERBB4/PIK3R1 split TEV assay in PC12 cells. ERBB4 was fused to NTEV-tevS-GV-2HA. PIK3R1 was fused to CTEV-2HA. ERBB4 dimerises after stimulation with indicatednumbers of Nrg1-typel- or Nrg1-typelll-expressing PC12 cells, then recruiting PIK3R1.

wt

Nrg1-typel- $\beta 1$ a

Nrg1-typellI- $\beta 1$ a
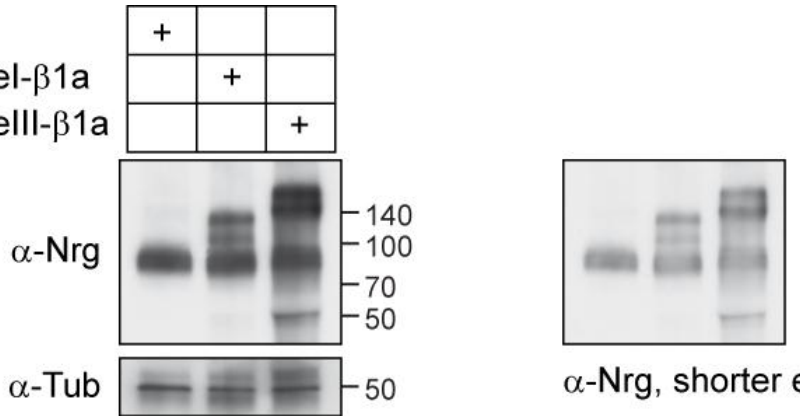

$\alpha$-Nrg, shorter exposure

Figure 27: Western blot analysis showing stable expression of, Nrg1-typel and Nrg1typelll in PC12 cells

Stable PC12 cell lines were generated using plasmids V368 (Nrg1-typel- $\beta 1$ a) and V370 (Nrg1-typelll- $\beta 1 \mathrm{a})$. Plasmids were not linearised before transfection. Co-selection was performed using the Hygromycin-containing plasmid pMSCV-hyg, with a dilution of 1:10 (1 part pMCV-hyg, 9 parts Nrg plasmids) and $100 \mu \mathrm{g} / \mathrm{ml}$ hygromycin. Maintenance of stable cells was done with $50 \mu \mathrm{g} / \mathrm{ml}$ hygromycin in regular PC12 medium. Western blots were probed with indicated antibodies, $\alpha$-Nrg targets the $\beta 1$ a tail of $\mathrm{Nrg} 1$. 
Results

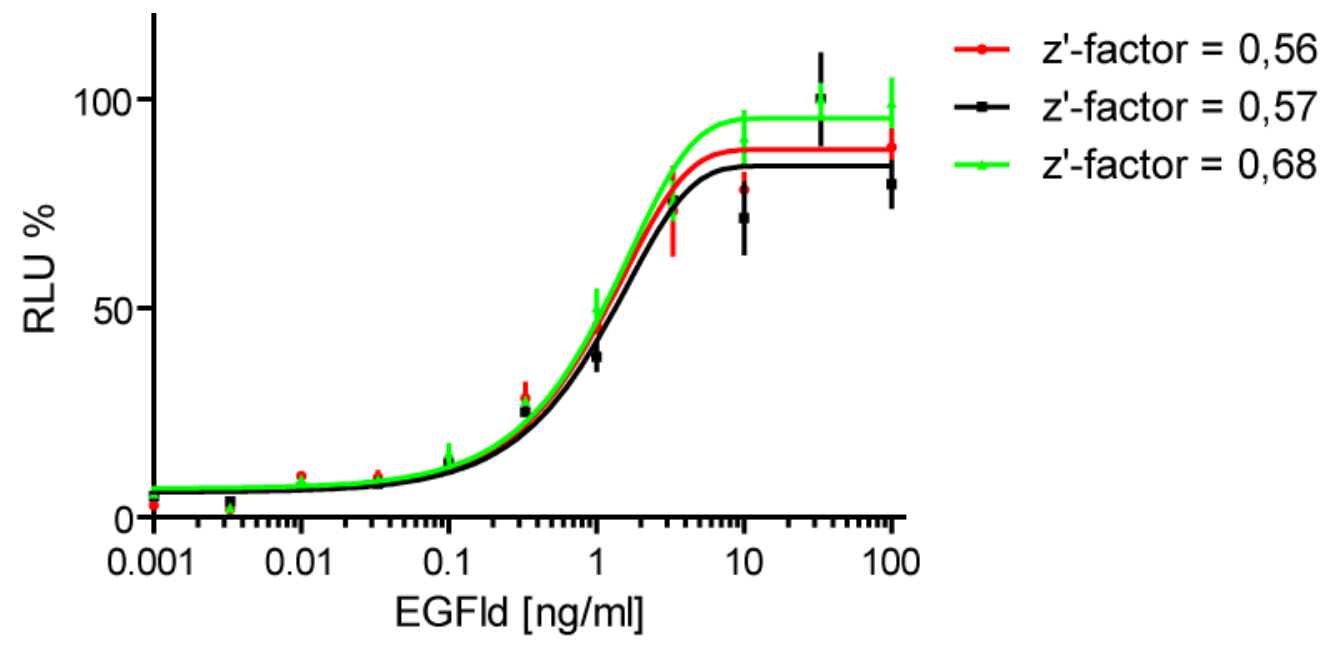

Figure 28: Robustness of the assay

Dose response of the ERBB4/PIK3R1 co-culture assay using increasing amounts of EGFlike domain. Comparison of three plate. ERBB4/PIK3R1 split TEV assay in PC12 cells. ERBB4 was fused to NTEV-tevS-GV-2HA. PIK3R1 was fused to CTEV-2HA. 
A

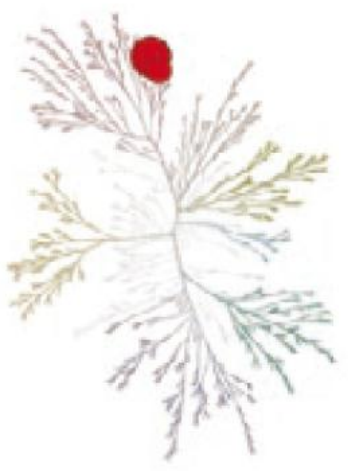

Lapatinib: kD 54nM

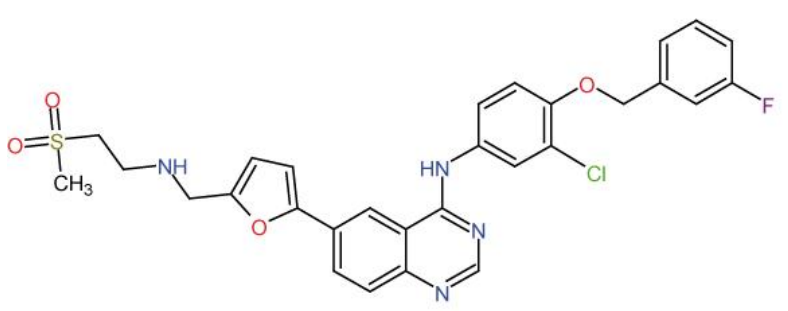

C

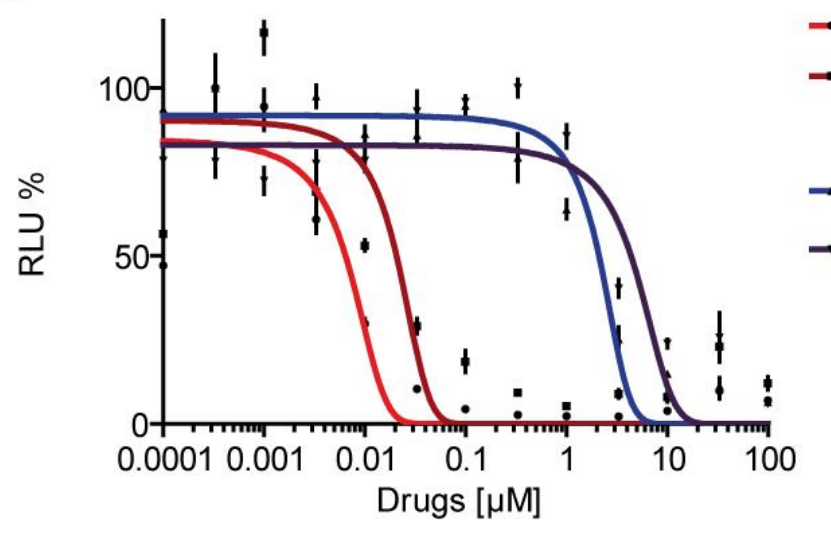

B

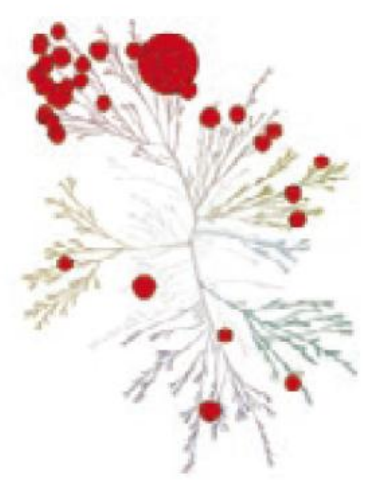

Cl-1033: kd 29nm<smiles>C=CC(=O)Nc1cc2c(Nc3ccc(F)c(Cl)c3)ncnc2cc1OCCCN1CCOCC1</smiles>

Cl-1033 EGFId

$\mathrm{Cl}-1033$

10k Nrg1-typel cells

- Lapatinib EGFId

- Lapatinib

10k Nrg1-typel cells

Figure 29: ERBB4 kinase inhibitor controls, Lapatinib and Cl-1033

A) Lapatinib is described as an ERBB-specific kinase inhibitor. The image shows the specificity of Lapatinib against the kinases within the human kinome tree (Karaman et al., 2008). The in-vitro kD of Lapatinib on ERBB4 is $54 \mathrm{nM}$. Chemical structure of Lapatinib (Karaman et al., 2008 supplements; Medina and Goodin, 2008). B) Cl-1033 is described as a kinase inhibitor not specific to ERBBs. The image shows the specificity of $\mathrm{Cl}-1033$ against the kinases within the human kinome tree (Karaman et al., 2008). The in-vitro kD of $\mathrm{Cl}-1033$ on ERBB4 is 29nM. Chemical structure of $\mathrm{Cl}-1033$ (Karaman et al., 2008 supplements; Slichenmyer and Fry). C) Comparison of the effects of Lapatinib and $\mathrm{Cl}$ 1033 on the ERBB4/PIK3R1 split TEV assay in PC12 cells. ERBB4 was fused to NTEVtevS-GV-2HA. PIK3R1 was fused to CTEV-2HA. 


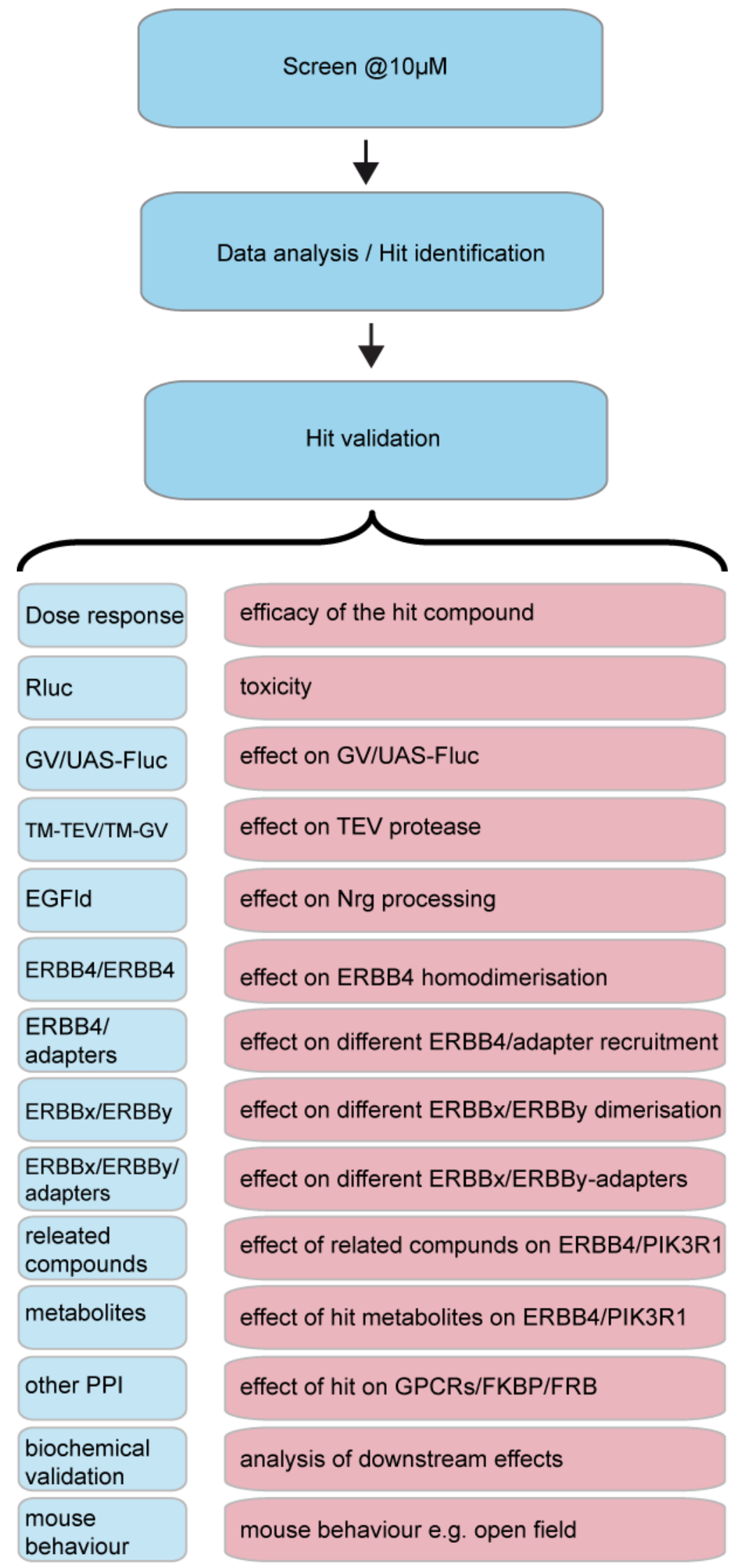




\subsection{The NCC201 screen}

The NCC201 library was screened according to the described parameters. 5,000 (Figure 30) and 10,000 (Figure 31) PC12 Nrg1-typel cells were used for the stimulation. A $z^{\prime}$-factor of $>0.5$ was obtained when full activation was triggered using EGF-like domain and inhibition using Lapatinib and $\mathrm{Cl}-1033$. Both screens resulted in one putative activator and one putative inhibitor. The inhibitor was identified to be Spironolactone. It causes a highly significant decrease of the luciferase activity in the screen $(p<0.0001)$. The activator was identified to be Albendazole. It causes a highly significant increase in luciferase activity $(p<0.0001)$. Both hits were recovered from both types of screens, either run with 5,000 or 10,000 Nrg1-typel-expressing PC12 cells. In addition, both hits showed no toxic effect in the GV-UAS-Fluc technical control screen.

The inhibitor Spironolactone is also found in a screen stimulated to maximum levels using the EGF-like domain, indicating a strong effect of the drug towards ERBB4 receptor activation, but not Nrg1-typel processing as the EGF-like domain already represents a form that is most possibly reduced in structure but retains highest biological activity of $\mathrm{Nrg} 1$ (Figure 33). 

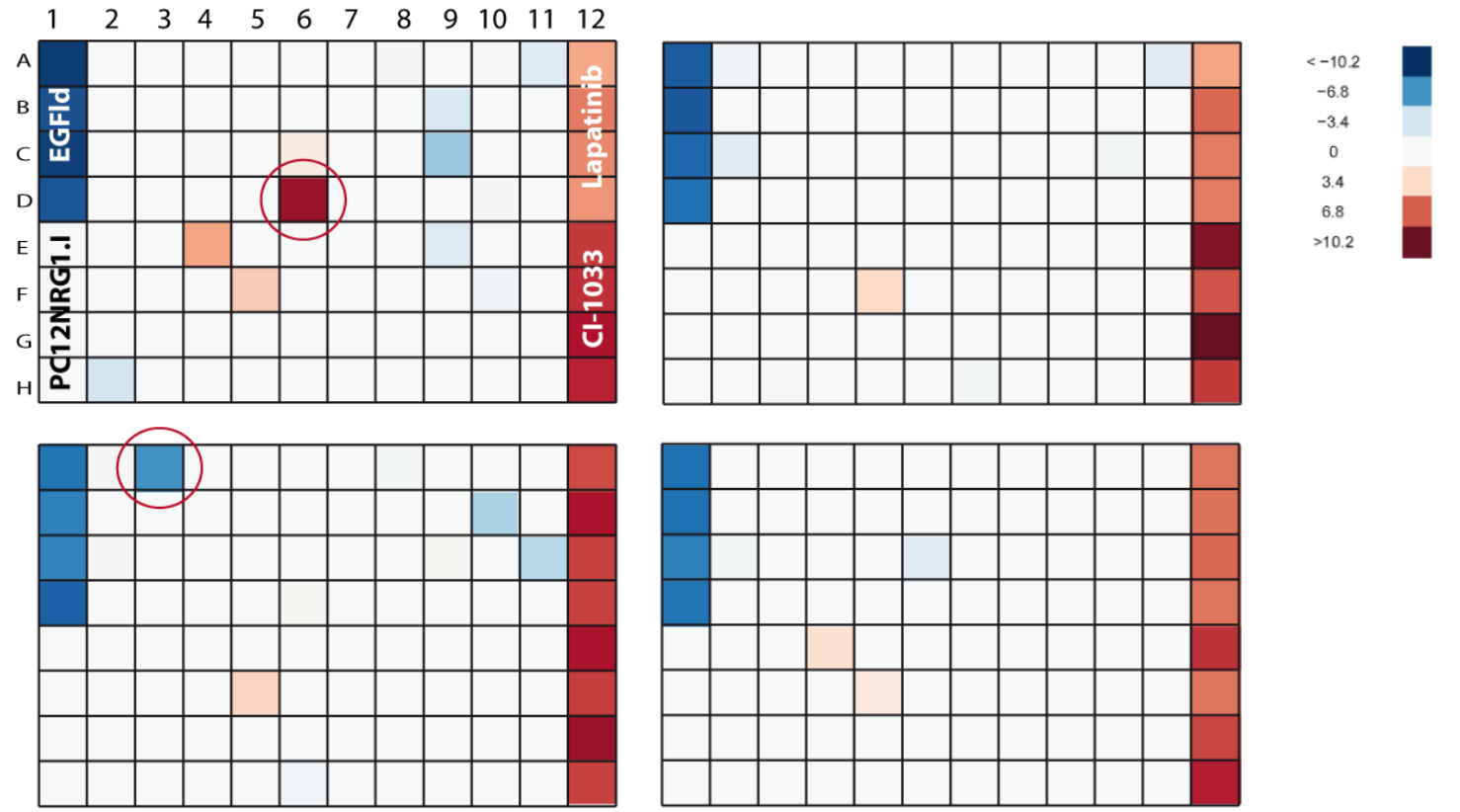

Top inhibitor Plate 1 D06 Spironolactone score 9,06

Top activator Plate $3 \mathrm{~A} 3$ Albendazole score $-6,76$

B

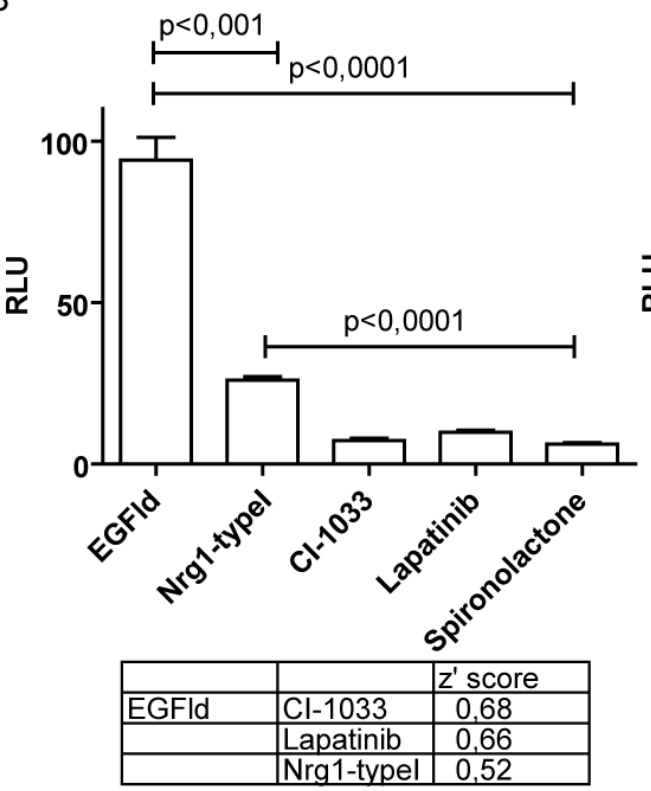

C

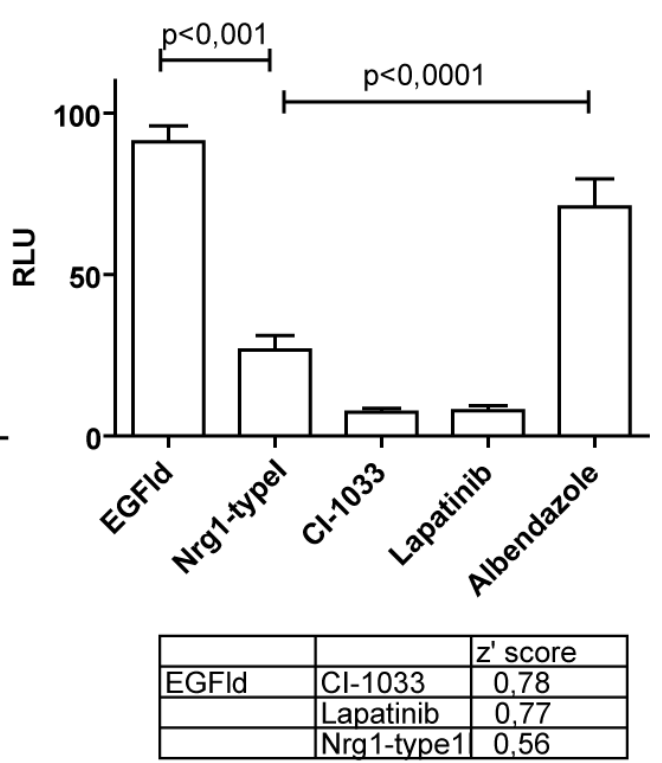

Figure 30 Screen of the NCC201 library

A) ERBB4/PIK3R1 split TEV based screening assay in PC12 cells. ERBB4 was fused to NTEV-tevS-GV-2xHA; PIK3R1was fused to CTEV-2HA. ERBB4 dimerises after stimulation with $5.000 \mathrm{Nrg} 1$-typel cells and recruit PIK3RI. Cells were further stimulated with EGFld $(10 \mathrm{ng} / \mathrm{ml})$, Lapatinib $(10 \mu \mathrm{M}), \mathrm{Cl}-1033(0.1 \mu \mathrm{M})$, or 80 different compounds (see plate design). Luciferase data was analysed using tinR (see script in the methods). B) Extracted data from the screen showing the significance of the effect of the top inhibitor Spironolactone. C) Extracted data from the screen showing the significance of the effect of the top activator Albendazole (Two paired, two sided TTest). 
$\begin{array}{llllllllllll}1 & 2 & 3 & 4 & 5 & 6 & 7 & 8 & 9 & 10 & 11 & 12\end{array}$
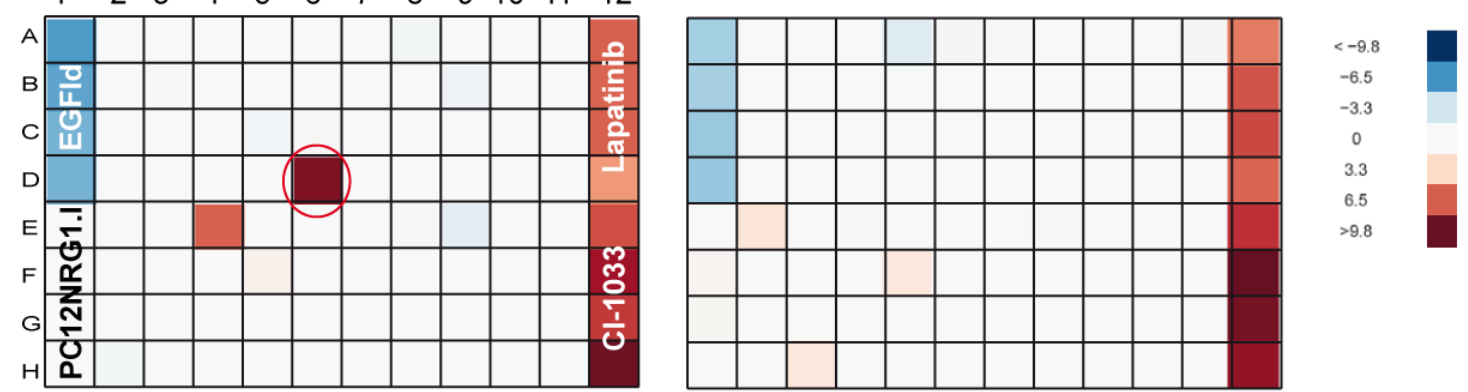

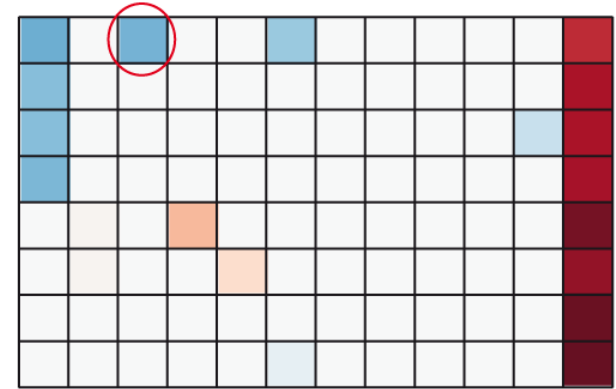

Top inhibitor Plate 1 D06

Spironolactone score 9,25

B

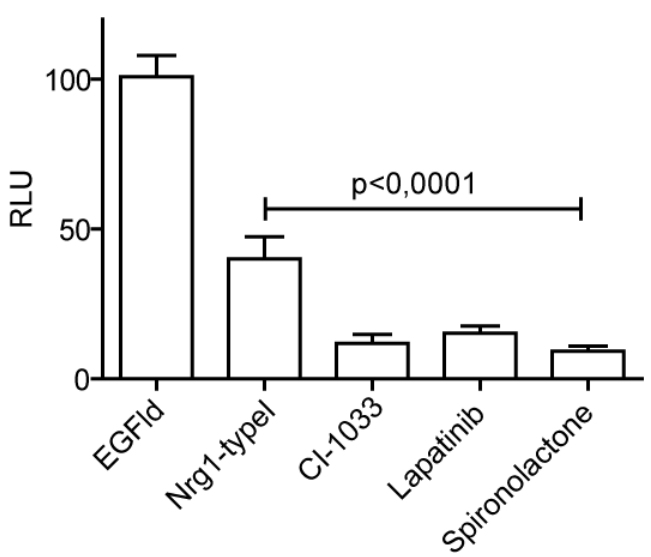

\begin{tabular}{|l|l|r|}
\hline & & $z^{\prime}$ score \\
\hline EGFld & Cl-1033 & 0,66 \\
\hline & Lapatinib & 0,70 \\
\hline & Nrg1-typel & 0,28 \\
\hline
\end{tabular}

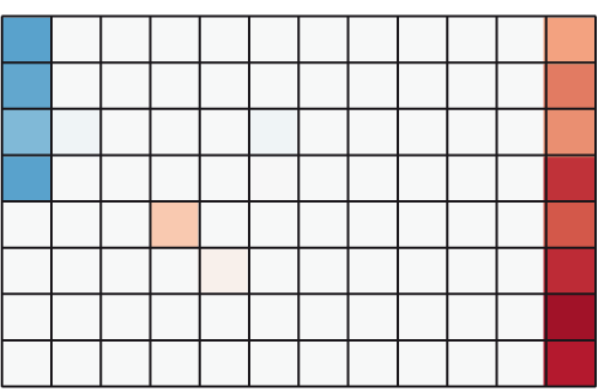

Top activator Plate $3 \mathrm{~A} 3$

Albendazole score $-5,48$

C
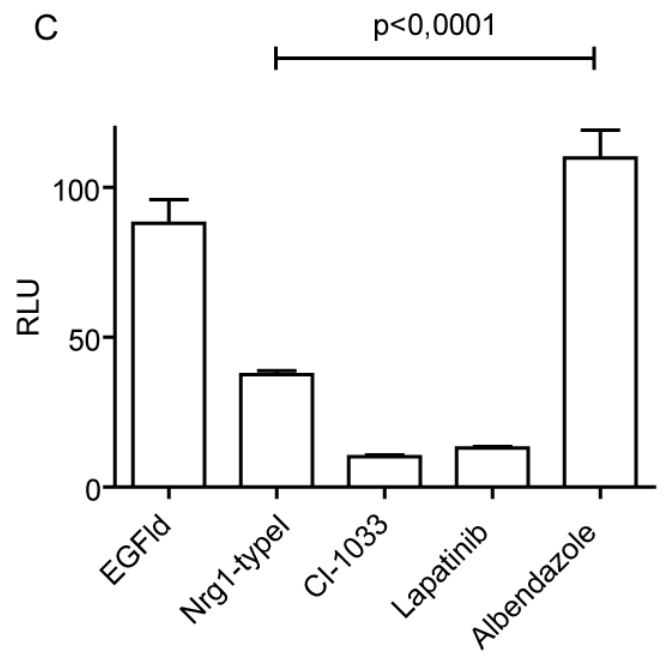

\begin{tabular}{|l|l|r|}
\hline & & z' score \\
\hline EGFId & Cl-1033 & 0,61 \\
\hline & Lapatinib & 0,61 \\
\hline & Nrq1-typel & 0,27 \\
\hline
\end{tabular}

Figure 31: Screen of the NCC201 library 10k Nrg1-typel cells

A) ERBB4/PIK3R1 split TEV based screening assay in PC12 cells. ERBB4 was fused to NTEV-tevS-GV-2xHA; PIK3R1was fused to CTEV-2HA. ERBB4 dimerises after stimulation with $10.000 \mathrm{Nrg} 1$-typel cells and recruit PIK3RI. Cells were further stimulated with EGFld $(10 \mathrm{ng} / \mathrm{ml})$, Lapatinib $(10 \mu \mathrm{M}), \mathrm{Cl}-1033(0.1 \mu \mathrm{M})$, or 80 different compounds (see plate design). Luciferase data was analysed using tinR (see script in the methods). B) Extracted data from the screen showing the significance of the effect of the top inhibitor Spironolactone. C) Extracted data from the screen showing the significance of the effect of the top activator Albendazole (Two paired, two sided TTest). 
Results

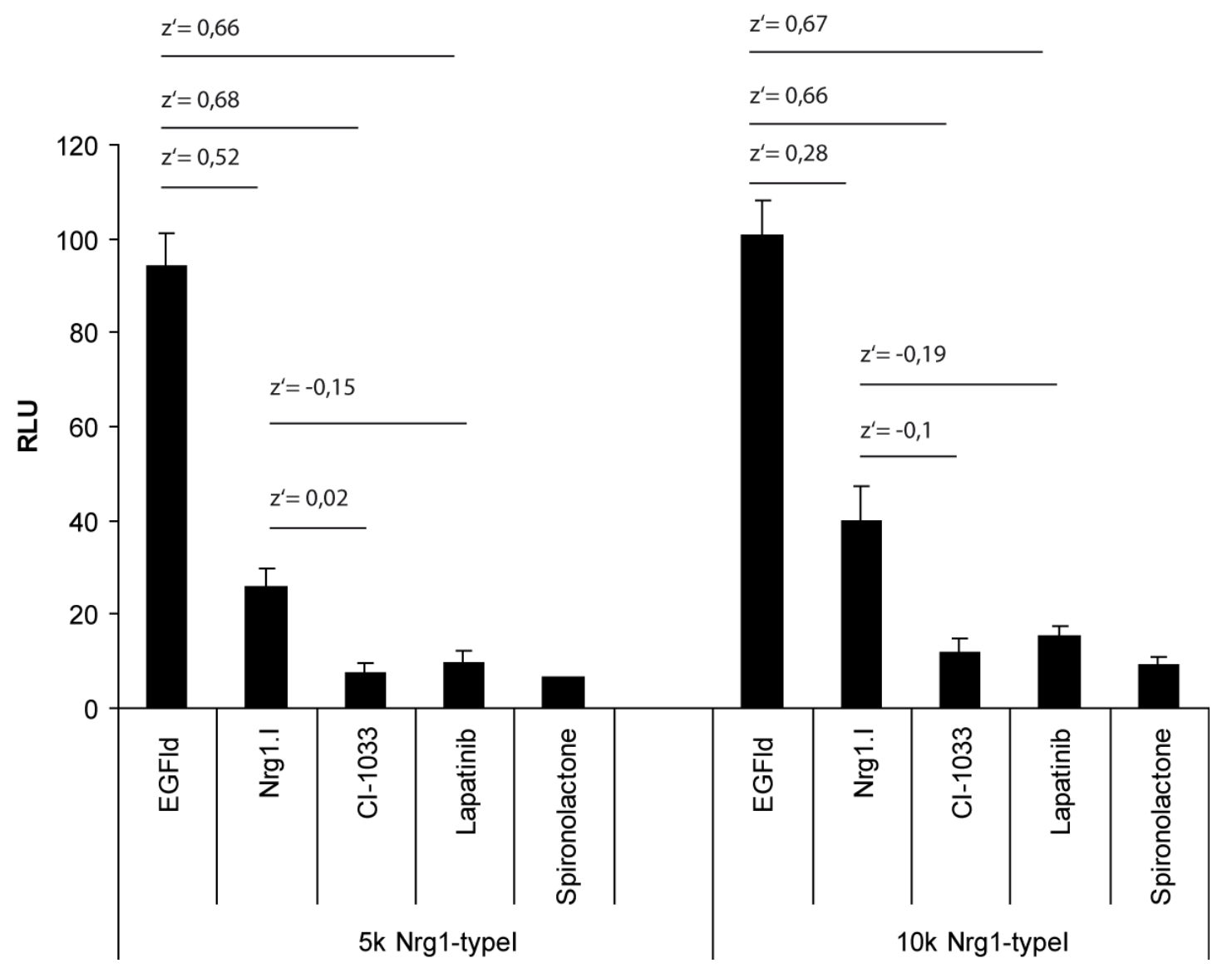

Figure 32: Detailed analysis of the functionality of the screen

$Z$ '-factors between the different activated and inhibited states of the assay. 
$\begin{array}{lllllllllllll}\mathrm{A} & 1 & 2 & 3 & 4 & 5 & 6 & 7 & 8 & 9 & 10 & 11 & 12\end{array}$
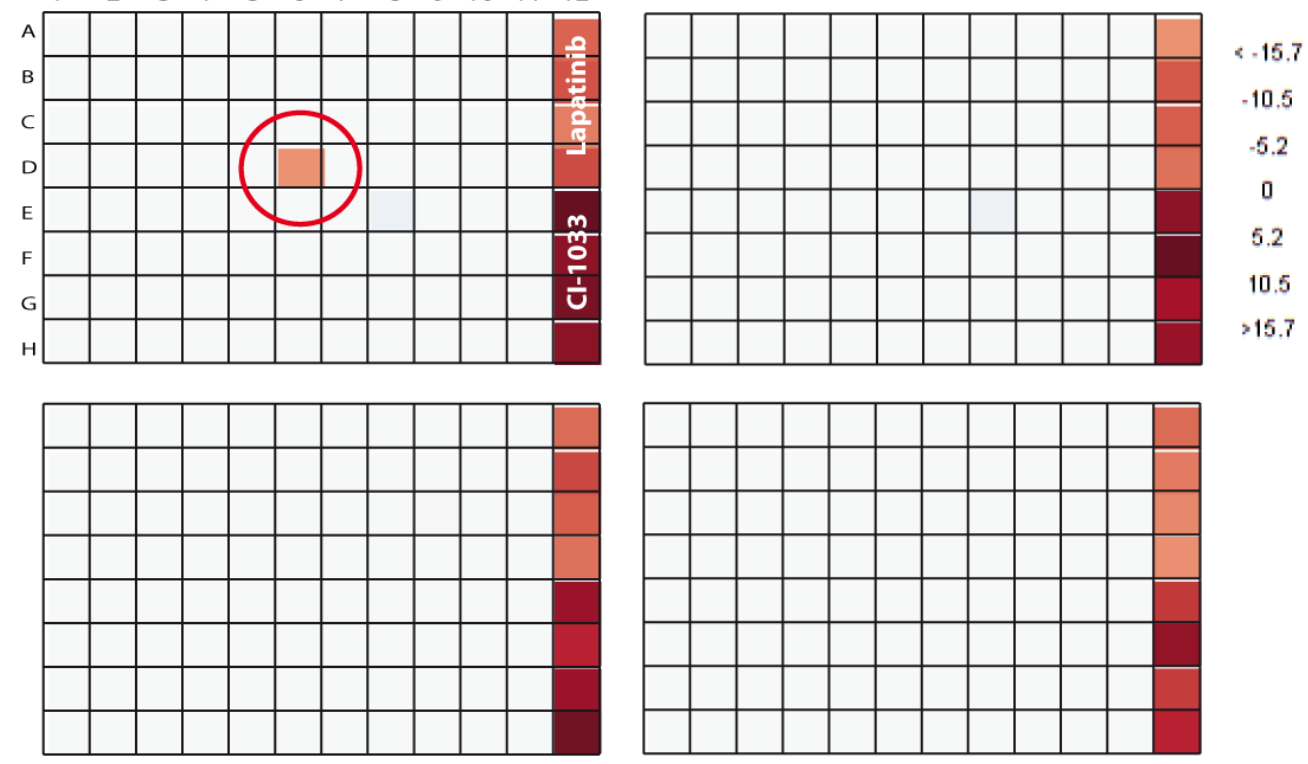

B

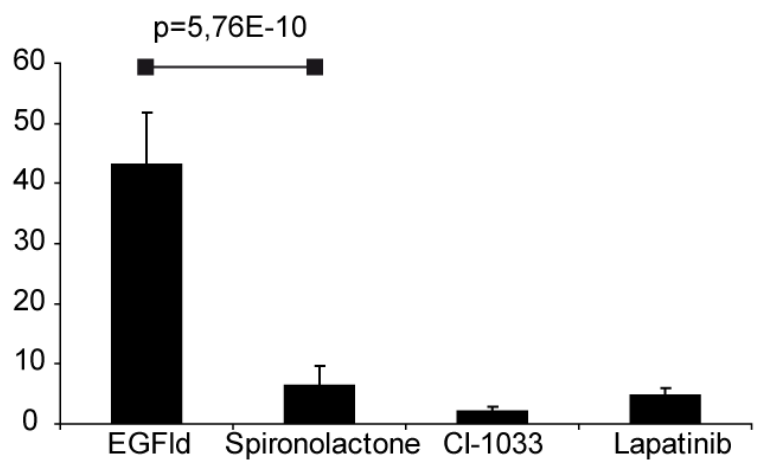

Figure 33: Counter-screen of the NCC201 library with EGF-like domain

A) ERBB4/PIK3R1 split TEV based screening assay in PC12 cells. ERBB4 was fused to NTEV-tevS-GV-2xHA; PIK3R1was fused to CTEV-2HA. ERBB4 dimerises after stimulation with $10 \mathrm{ng} / \mathrm{ml}$ EGFId and recruit PIK3RI. Cells were further stimulated with Lapatinib $(10 \mu \mathrm{M}), \mathrm{Cl}-1033(0.1 \mu \mathrm{M})$, or 80 different compounds (see plate design). Luciferase data was analysed using tinR (see script in the methods). B) Extracted data from the screen showing the significance of the effect of the top inhibitor Spironolactone. 

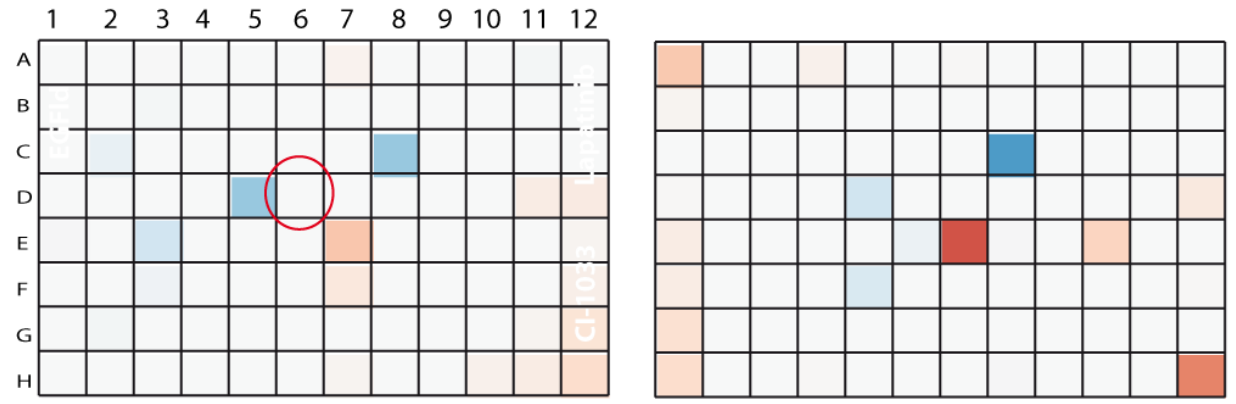

$<-8.6$
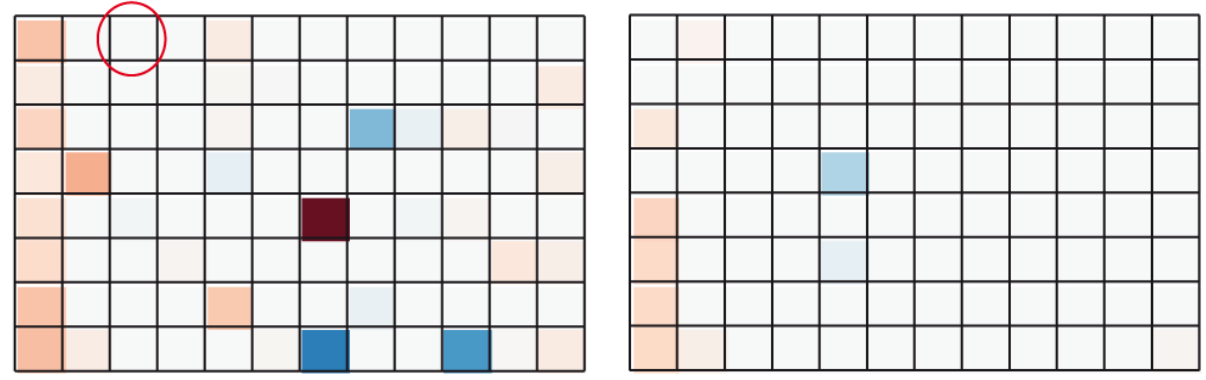

Plate 1 D06 Spironolactone

Plate 3 A03 Albendazole

\section{Figure 34: Counterscreen of the NCC201 library with GV}

PC12 cells were transfected with GV and stimulated with Lapatinib $(10 \mu \mathrm{M}), \mathrm{Cl}-1033$ $(0,1 \mu \mathrm{M})$, or 80 different compounds (see plate design). The red circles show that now effect on the UAS-firefly luciferase is measured with the indicated hits Spironolactone and Albendazole. 


\subsection{The NCC003 Screen}

The NCC003 library was screened according to the described parameters. 10,000 PC12 Nrg1-typel cells were used for the stimulation. A z'-factor of $>$ 0.5 was obtained when using EGFld for activation and the Lapatinib and $\mathrm{Cl}$ 1033 for inhibition. From this part of the library, Topotecan showed the strongest inhibiting effect.
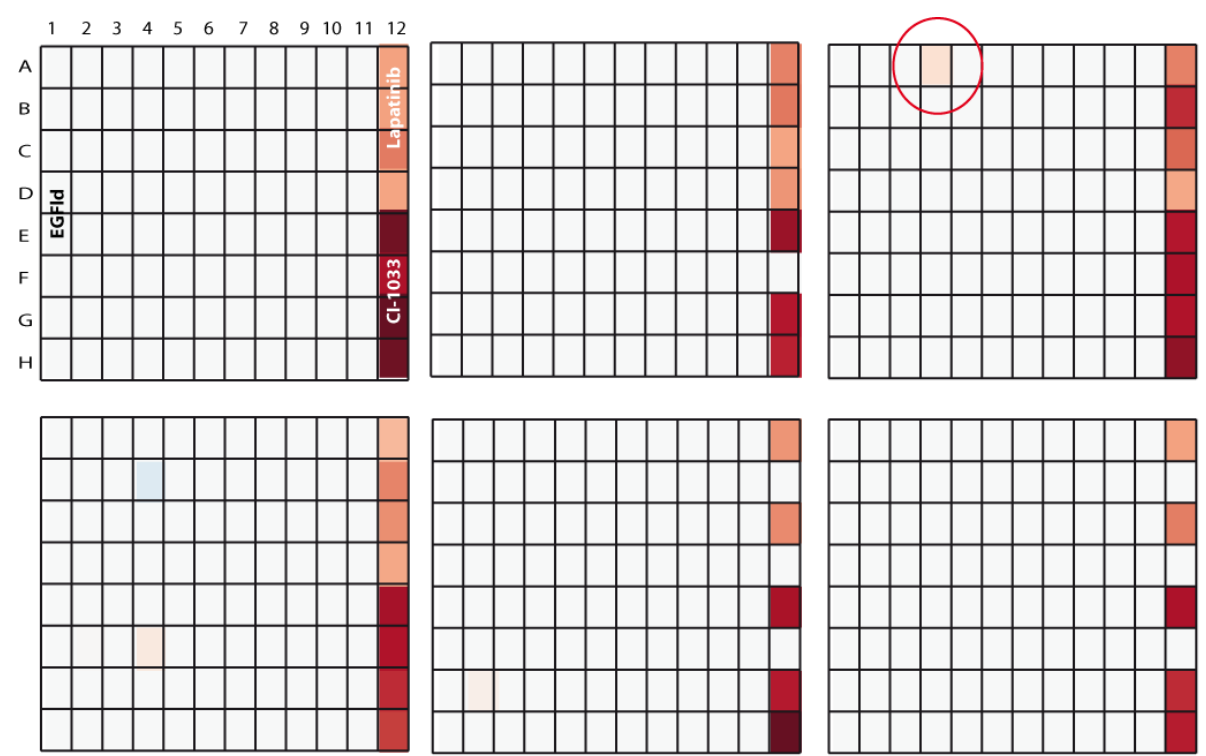

Figure 35: NCC003 screen

Split TEV-based screening assay in PC12 cells. ERBB4 was fused to NTEV-tevS-GV2HA. PIK3R1 was fused to CTEV-2HA. The complete assay was stimulated with $10 \mathrm{ng} / \mathrm{ml}$ EGFId. Lapatinib and $\mathrm{Cl}-1033$ are used as negative controls and inhibit kinase activity with a concentration of $10 \mu \mathrm{M}$ for Lapatinib and $0.1 \mu \mathrm{M}$ for $\mathrm{Cl}-1033(12 \mathrm{~A}-\mathrm{H})$. Topotecan is highlighted by a red circle (top right panel). 


\subsection{Hit validation}

From the NCC201 screen, two hits were recovered, the putative activator Albendazole and the putative inhibitor Spironolactone. From the NCC003 screen, one hit was recovered, the putative inhibitor Topotecan. During the development of the co-culture screen a couple of interesting substances showed alterations in the firefly activity, with minor statistical significance. These substances were chosen to test the validity of the validation concepts

\begin{tabular}{|l|l|l|}
\hline Name & Location & Putative function \\
\hline Spironolactone & NCC201 P1 D7 & Inhibitor \\
\hline Albendazole & NCC201P3 A3 & Activator \\
\hline Topotecan & NCC003 P3 A4 & Inhibitor \\
\hline Vincristine & NCC003 P6 B02 & Inhibitor \\
\hline Mevastatin & NCC003 P6 H4 & Inhibitor \\
\hline CCPA & NCC003 P6 B6 & Activator \\
\hline K252a test substance & Paper (Kuai et al. 2010) & Activator \\
\hline
\end{tabular}

Table 12: overview of candidate hits recovered from the screen (italics) and additionally validated substances

The hit validation is separated into three parts, named (I) vertical, (II) horizontal and (III) orthogonal validation.

(I) The vertical validation addresses at what level of the signalling cascade a candidate might be acting. In the co-culture assay, I tested whether the substance interfered with the localisation and processing of $\mathrm{Nrg} 1$, the binding of $\mathrm{Nrg} 1$ to the ERBB receptors, the homodimerisation of the receptors, the auto-phosphorylation of the receptors, the adaptor recruitment to the receptor, and all artificial components of the assay.

(II) The horizontal validation examines the specificity of the substances on Nrg1-ERBB4 signalling. Different forms of NRG1-typel, different homo and heterodimers of ERBB receptors, and different adapters of the ERBB system can be tested, resulting in more than 200 potential combinations to test.

(III) The orthogonal validation can be sub-divided further. First, the hit substances' effects on the downstream signalling cascades were addressed using other methods, such as phospho-specific antibodies. Second, the candidates were tested in split TEV assays using related (i.e. other receptors, such as the GPCR HTR5A) and unrelated interaction partners (i.e. the model 
Results

interaction of FRB and FKBP. Third, hit compounds were searched for structurally closely related substances, and their effects on the ERBB4/PIK3R1 co-culture assay were then analysed. Finally, first attempt towards the in vivo validation of the primary hit Spironolactone in a mouse model were undertaken. 


\subsection{Spironolactone}

Spironolactone is a synthetic, steroid like drug, which targets primarily the mineralocorticoid receptor (MCR), but it has also anti-androgen and progesterone properties. It acts as a competitive MCR antagonist (e.g. Aldosterone receptor).

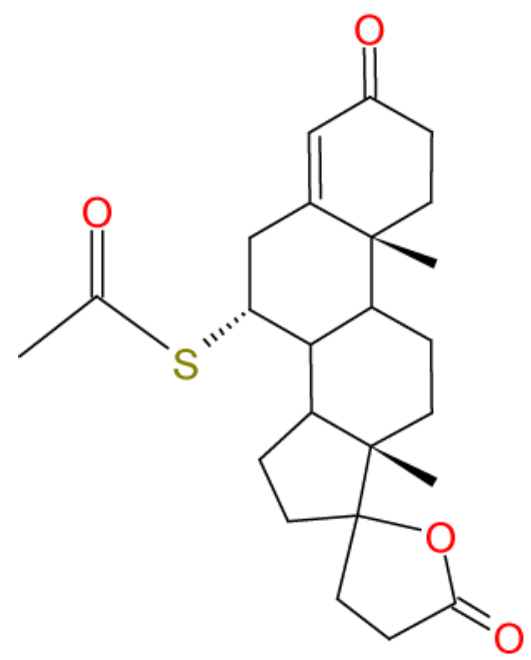

Figure 36: Spironolactone

The MCR is a cytosolic receptor bound to HSP90 proteins. After Aldosterone binding HSP90 proteins are released, causing the MCR to homodimerise and to translocate to the nucleus, where the MCR binds hormone response elements (Fuller et al., 2012; Pippal and Fuller, 2008). It has been shown that a Spironolactone-bound MCR does not translocate to the nucleus, as this inhibits the dimerisation of the receptor (Grossmann et al., 2012). A BRET study showed that a Spironolactone-bound MCR does not homodimerise, in contrast to a Aldosteronebound MCR (Grossmann et al., 2012).

Spironolactone is used as diuretic, antihypertensive, and anti-androgen (Ogden et al., 1961). The known target profile of Spironolactone has been reported to include a much broader spectrum than the MCR only. To date, the following pharmacological inhibition or activation of the following receptors have been described (Fagart et al., 2010):

\begin{tabular}{|l|l|}
\hline Receptor & IC $_{50}$ \\
\hline Mineralocorticoid receptor & $24 \mathrm{nM}$ \\
\hline Androgen receptor & $77 \mathrm{nM}$ \\
\hline Glucocorticoid receptor & $2.42 \mu \mathrm{M}$ \\
\hline Progesterone receptor & $0.74 \mu \mathrm{M}$ \\
\hline
\end{tabular}

Table 13. IC $\mathrm{C}_{50}$ values of various targets of Spironolactone (Fagart 2010)

The half-life of Spironolactone is difficult to determine (Brittain, Analytical Profiles of Drug Substances and Excipients). It is rapidly metabolised in the liver, and more than 17 metabolites were isolated from humans. It is unclear whether one or more of these metabolites are responsible for the effects observed in pharmacological studies. Spironolactone also was reported to 
mildly deteriorate depressive behaviour when combined with the antidepressant drug amitriptyline (Holsboer, 1999).

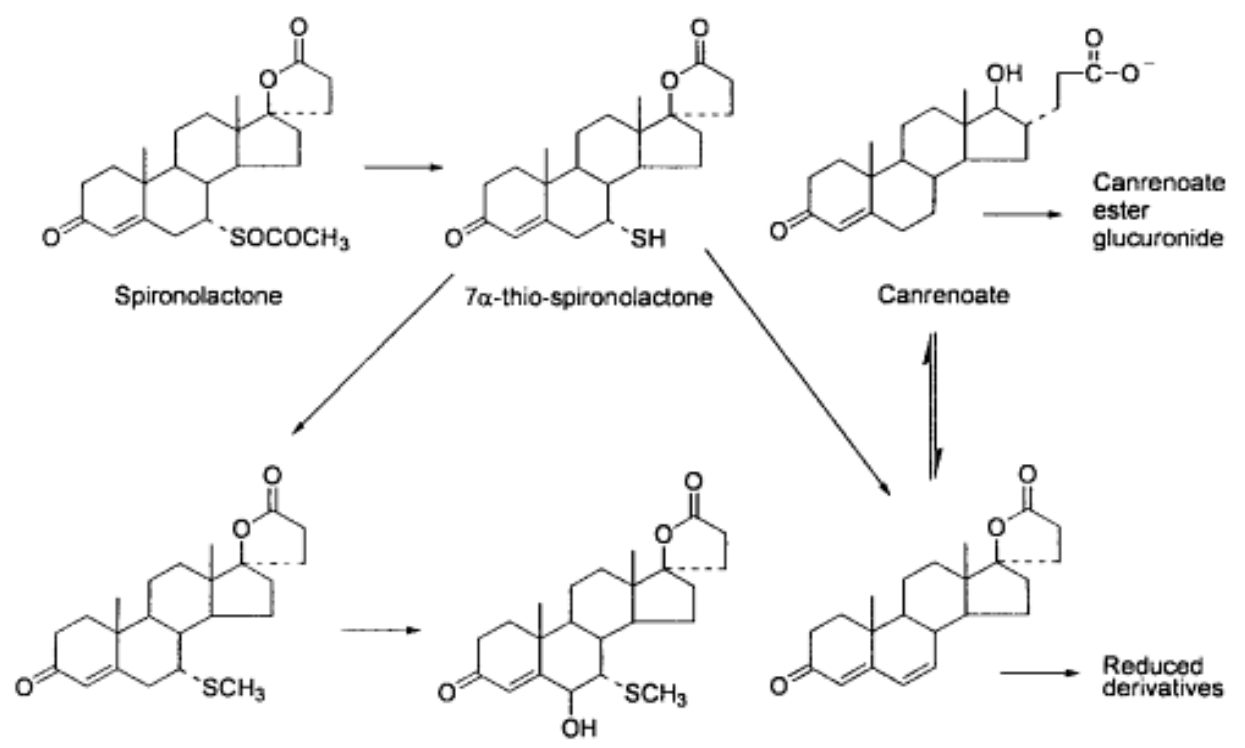

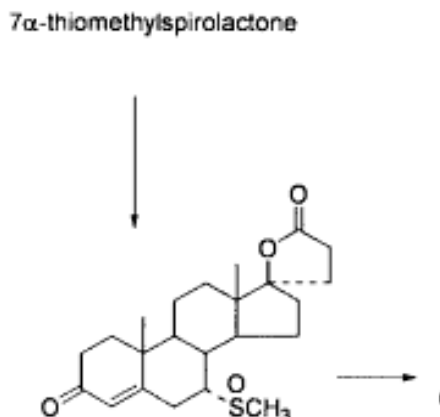

$6 \beta-\mathrm{OH}-7 \alpha$-thiomethyl-

Canrenone spirolactone

$7 \alpha$-methylsulfinylspirolactone<smiles></smiles><smiles>CC12CCC(=O)C=C1C=CC1C2C(O)CC23OC(=O)CCC2C12CCCC32C</smiles>

$6 \beta-\mathrm{OH}-7 \alpha-$ methylsulfinylspirolactone

$15 \alpha-\mathrm{OH}$-canrenone and possible other hydroxylated derivatives

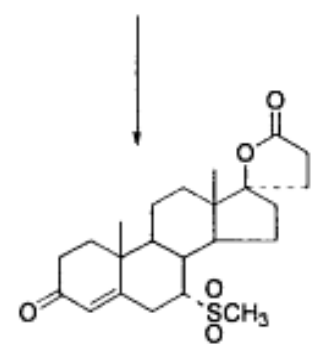

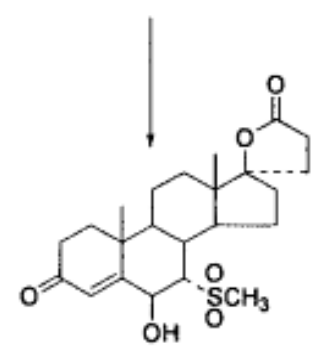

$7 \alpha$-methylsulfonylspirolactone

$6 \beta-\mathrm{OH}-7 \alpha-$ methylsuifonylspirolactone

\section{Figure 37: Map Spironolactone metabolites}

Spironolactone is processed in the liver and metabolised into 17 metabolites. It is speculated that these metabolites also have an effect on the MCR, and may have other unknown functions as well. It is not known whether the structural integrity of Spironolactone is kept in a co-culture assay without liver processing (Analytical Profiles of Drug Substances and Excipients 2002 Harry G. Brittain). 
In the screens performed, Spironolactone was found to decrease Nrg1ERBB4 signalling with varying but consistent levels (5,000 and 10,000 PC12 Nrg1-typel cells). Spironolactone was further validated in the secondary screening process where $10 \mathrm{ng} / \mathrm{ml}$ EGF-like domain was used to stimulate ERBB4 signalling. The GV control screen showed that Spironolactone is not toxic for the cells at screening concentrations.

Next, possible modes of actions for Spironolactone were analysed. To do this, a vertical validation approach was used that addressed the pathway from upstream to downstream components including technical controls. In addition, a horizontal validation approach was initiated to investigate potential functions for other NRG isoforms, ERBB receptors, and adapter molecules, such as GRB2 and SHC1.

\subsubsection{Vertical validation for Spironolactone}

An inhibitory dose response assay was established for Spironolactone. The dose response to increasing concentrations of Spironolactone in the Nrg1typel-ERBB4/PIK3R1 co-culture assay showed an IC50 of $0.9 \mu \mathrm{M}$ (

Figure 38).

\subsubsection{Technical controls for Spironolactone validation}

\subsubsection{Renilla luciferase}

TK-Rluc reported the toxicity of Spironolactone. Spironolactone is toxic for the cells in this assay at concentrations at an $\mathrm{IC}_{50}$ of $56.4 \mu \mathrm{M}$. Figure 39

\subsubsection{The Gal4-VP16 control assay}

In a first technical control assay, UAS-Fluc was co-transfected with GV only. This setup showed the effect of Spironolactone on the GV/UAS reporter system and the firefly luciferase. The $\mathrm{IC}_{50}$ of Spironolactone in this assay is at a concentration of $10.7 \mu \mathrm{M}$ (Figure 39).

\subsubsection{TEV protease control assay}

To correct for any effects elicited by the TEV protease and its activity at TEV protease cleavage sites, a transmembrane version of the TEV protease (TMTEV) was co-transfected with a transmembrane-bound GV carrying an internal TEV cleavage site (TM-GV), along with UAS-Fluc and Rluc reporters. The $\mathrm{IC}_{50}$ for Spironolactone in this constitutively activated TEV protease assay was determined to be at a concentration of $24.3 \mu \mathrm{M}$. When comparing this $I_{50}$ value with the one obtained from the $G V$ control assay $\left(\mathrm{IC}_{50}, 10.7 \mu \mathrm{M}\right)$, 
I can conclude that Spironolactone most likely has no effect on TEV protease activity (Figure 39).

\subsubsection{1-cell assay with soluble Nrg1-EGF-like domain}

To test whether the Spironolactone effect is targeted against Nrg1-typel expression or processing, the Nrg1-typel-expressing PC12 cells were exchanged for the soluble EGF-like domain. The EGF-like domain was used as a surrogate for any processed Nrg1 isoforms. The replacement of Nrg1typel with EGF-like domain showed no functional differences in the performance of the assay, suggesting that Spironolactone does not target full length Neuregulin processing and activity (Figure 39).

The adapter proteins PIK3R1 and SRC are primarily targeted by Spironolactone

The effect of Spironolactone on the recruitment of various other adapter proteins was analysed. In the split TEV-based assay, SHC1, STAT5A and GRB2 showed an $\mathrm{IC}_{50}$ of roughly $5 \mu \mathrm{M}, \mathrm{SRC}$ and PIK3R1 displayed an $\mathrm{IC}_{50}$ of about $0.7 \mu \mathrm{M}$ Spironolactone. These data suggest that in particular the binding of the adapter molecules PIK3R1 and SRC to the ERBB4 receptor is inhibited by Spironolactone (Figure 40, Figure 41). 


\subsubsection{Horizontal validation}

\section{Dimerisations of the ERBB receptor family}

\subsubsection{ERBB4 receptor dimerisations}

A prerequisite for ERBB4 receptor activation is the dimerisation with itself or ERBB2, which is the preferred partner for all ERBB receptors. Therefore, split TEV assays were setup consisting of an ERBB4-NTEV-tevS-GV cotransfected with either ERBB2-CTEV or ERBB4-CTEV. Efficient activation was tested using EGF-like domain (Figure 42,Figure 43), followed by doseresponse analyses for Spironolactone on the ERBB2/4 heterodimerisation and ERBB4 homodimerisation. The ERBB2/4 dimerisation yielded an $\mathrm{IC}_{50}$ $2.2 \mu \mathrm{M}$, and the ERBB4 homodimerisation showed an $\mathrm{IC}_{50}$ of $2.4 \mu \mathrm{M}$ Spironolactone.

\subsubsection{ERBBX/ERBBy}

In addition, a complete panel of ten different ERBB receptor homo- and heterodimers stimulated with $10 \mathrm{ng} / \mathrm{ml}$ EGF-like domain was measured. All combinations tested showed an $\mathrm{IC}_{50}$ of $2.2 \mu \mathrm{M}$ to $9.2 \mu \mathrm{M}$ Spironolactone (see table 14 below). A comparison to the ERBB4/PIK3R1 assay clearly showed a bias towards a selective inhibition for the adapter assay involving PIK3R1 (Figure 42).

\subsubsection{ERBB2/ERBB4 dimerisation and adapters}

To get further insight of Spironolactone's specificity towards the potential inhibition of ERBB2 and ERBB4, the adapter recruitment for ERBB2/4 heterodimers was analysed. The adapter recruitment of ERBB2/ERBB4 showed $\mathrm{IC}_{50}$ values between $0.6 \mu \mathrm{M}$ and $1.7 \mu \mathrm{M}$ (Figure 46 ).

\subsubsection{ERBB2/ERBB3 dimerisation and adapters}

Likewise, the adapter recruitment for ERBB2/3 heterodimers was measured. The analysis showed $I_{50}$ values between $0.5 \mu \mathrm{M}$ and $0.9 \mu \mathrm{M}$. The comparison of the datasets obtained with ERBB2/3 and ERBB2/4 indicates that the effect of Spironolactone is not ERBB4-specific, but also inhibits ERBB3-mediated adaptor recruitment assays Figure 45.

\subsubsection{ERBB4/PIK3R1 assay and effects caused by other Aldosterone derivatives}

To get further insight into Spironolactone's specificity, the data from the NCC201 and the NCC003 screens were analysed for Aldosterone derivatives and structures related to Spironolactone. 25 structural related drugs were 
identified. None of these drugs showed a similarly efficient inhibitory effect on the ERBB4/PIK3R1 co-culture assay (Figure 49)

\subsubsection{ERBB4/PIK3R1 assay and effects caused by Eplerenone and Canrenone}

Eplerenone, the second generation substance of Spironolactone used in the clinics, was also tested in the ERBB4/PIK3R1 assay stimulated with EGF-like domain. Eplerenone is structural and functional closely related to Spironolactone but did not show any effect in the ERBB4/PIK3R1 assay (Figure 48).

Canrenone, a metabolite of Spironolactone lacking the thioketone group was also tested in a similar ERBB4/PIK3R1 assay and showed also no specific inhibiting effect (Figure 48).

The analysis of Eplerenone and the Aldosterone derivatives suggests that the measured effect is Spironolactone-specific and not a general side effect caused by other MCR antagonists or Aldosterone derivatives (Figure 49).

\subsubsection{Orthogonal validation}

\subsubsection{ERBB1/ERBB1/EGF}

Spironolactone showed an effect on EGF-stimulated ERBB1/ERBB1 homodimerisation, with an $\mathrm{IC}_{50}$ of $1.1 \mu \mathrm{M}$ Spironolactone (Figure 50 ).

To address whether Spironolactone is specific to the inhibition of ERBB receptor-mediated signalling, I tested Spironolactone's effect in various other split TEV assays that also included the regulated activation of a G-proteincoupled receptor (GPCR).

\subsubsection{GPCR Serotonin receptor 5A (HTR5A) activation}

Spironolactone showed no effect on the Serotonin-stimulated HTR5A- $\beta$ arrestin (ARRB2) split TEV assay (Figure 51).

\subsubsection{FRB/FKBP model interaction induced by Rapamycin}

Spironolactone showed a minor effect on the Rapamycin-induced FRB/FKBP interaction, with an $\mathrm{IC}_{50}$ of $18.8 \mu \mathrm{M}$ (Figure 52). 
5.6.5 Summary IC $_{50}$ validation Spironolacone

\begin{tabular}{|c|c|c|c|}
\hline & & & $I_{50}$ in $\mu \mathrm{M}$ \\
\hline & Rluc & & 56.4 \\
\hline & GV & & 10.7 \\
\hline TM-TEV & TM-GV & & 24.3 \\
\hline NTEV-tevS-GV & CTEV & & $I C_{50}$ in $\mu \mathrm{M}$ \\
\hline ERBB4 & ERBB4 & & 2.4 \\
\hline \multirow[t]{2}{*}{ ERBB3 } & ERBB4 & & 4.4 \\
\hline & ERBB3 & & 3.1 \\
\hline \multirow[t]{3}{*}{ ERBB2 } & ERBB4 & & 2.2 \\
\hline & ERBB3 & & 3.2 \\
\hline & ERBB2 & & 9.2 \\
\hline \multirow[t]{4}{*}{ ERBB1 } & ERBB4 & & 4.3 \\
\hline & ERBB3 & & 2.9 \\
\hline & ERBB2 & & 5.8 \\
\hline & ERBB1 & & 6.0 \\
\hline \multirow[t]{6}{*}{ V5 tag } & NTEV-tevS-GV & CTEV & $\mathrm{IC}_{50}$ in $\mu \mathrm{M}$ \\
\hline & ERBB4 & SHC1 & 4.9 \\
\hline & & GRB2 & 4.8 \\
\hline & & SRC & 0.7 \\
\hline & & PIK3R1 & 0.7 \\
\hline & & STAT5A & 4.6 \\
\hline \multirow[t]{5}{*}{ ERBB2 } & ERBB3 & SHC1 & 0.9 \\
\hline & & GRB2 & 0.6 \\
\hline & & SRC & 0.9 \\
\hline & & PIK3R1 & 0.5 \\
\hline & & STAT5A & 0.7 \\
\hline \multirow[t]{5}{*}{ ERBB2 } & ERBB4 & SHC1 & 0.8 \\
\hline & & GRB2 & 0.8 \\
\hline & & SRC & 1.8 \\
\hline & & PIK3R1 & 0.8 \\
\hline & & STAT5A & 0.7 \\
\hline NTEV-tevS-GV & CTEV & Drug & \\
\hline ERBB1 & ERBB1 & EGF & 1.1 \\
\hline ERBB4 & PIK3R1 & co-culture assay & 0.9 \\
\hline ERBB4 & PIK3R1 & Eplerenone & n.c. \\
\hline ERBB4 & PIK3R1 & Canrenone & n.c. \\
\hline FRB & FKBP & Rapamycin & 18.8 \\
\hline
\end{tabular}

Table 14. Summary of $\mathrm{IC}_{50}$ values 
These data suggest that Spironolactone affects the complete family of ERBB receptors, with a biased activity profile towards more efficient inhibition of adapter recruitment, particularly PIK3R1 and SRC.

\subsubsection{Biochemistry}

The biochemical, or orthogonal, validation of Spironolactone compared to Lapatinib showed the following effects:

\begin{tabular}{|l|l|l|l|l|}
\hline Antibody & Lapatinib & $\begin{array}{l}\text { Lapatinib } \\
\text { EGFId }\end{array}$ & Spironolactone & $\begin{array}{l}\text { Spironolactone / } \\
\text { EGFId }\end{array}$ \\
\hline $\begin{array}{l}\text { ERBB4 } \\
\text { (Buonanno) }\end{array}$ & & & & \\
\hline $\begin{array}{l}\text { ERBB4 (Santa } \\
\text { Cruz) }\end{array}$ & & activated & & partial degradation \\
\hline $\begin{array}{l}\text { pERBB4 Y1284 } \\
\text { (SHC) }\end{array}$ & & inhibited & & \\
\hline $\begin{array}{l}\text { pERBB4 } \\
\text { Y1056(PIK3) }\end{array}$ & inhibited & & partially inhibited \\
\hline $\begin{array}{l}\text { pERBB4 Y984 } \\
\text { (STAT5A) }\end{array}$ & & inhibited & & \\
\hline S6K & & & & \\
\hline pS6K & & inhibited & & \\
\hline ERK1 & & & \\
\hline pERK1 \\
T202/Y204
\end{tabular}

Table 15. Results of the biochemical validation using phospho-specific antibodies

The validation shows a clear difference between Lapatinib and Spironolactone effects. Lapatinib inhibits the phosphorylation at the three adapter docking sites Y1284 (SHC1), Y1056 (PIK3R1), and Y984 (STAT5A). Spironolactone has only a mild effect on the phosphorylation at the PIK3R1 docking site Y1056.

Lapatinib inhibits phosphorylation of ERK1 independent of EGF-like domain stimulation. Spironolactone, however, has no effect on the phosphorylation levels of ERK1.

The data suggests a difference in the mode of action of Spironolactone and the Lapatinib (Figure 54).

\subsubsection{Validation in HEK293 cells}

Western blot analysis on HEK293 cell lysates using phospho-specfic antibodies showed a similar effect of Lapatinib and Spironolactone on the phosphorylation levels of ERBB4 at Y1284 (Figure 53). 


\subsubsection{Validation in a MK801 mouse model of psychosis}

MK801 is an antagonist of the NMDA-type Glutamate receptor and has been shown to induce psychosis/schizophrenia-related behaviours including hyperactivity in rodents when applied experimentally (Eyjolfsson et al., 2006). The mode-of-action of MK801 is unclear but it has been suggested to act mainly on NMDA receptors located on interneurons thereby altering the balance of excitation and inhibition (Figure 20).Thus, MK801 and the Nrg1ERBB4 signalling may modulate similar cellular circuits affected in psychiatric diseases. To assess the potential impact of the novel Nrg1-ERBB4 inhibitor spironolactone on mouse behaviour, we applied the MK801 model of druginduced psychosis.

Mice were administered with drugs following the protocol depicted in (Figure $55 \mathrm{~A})$. Their locomotor behavior in the open field, i.e. distance travelled, time which mice spent active, time spent in the centre of the test arena, and the number of rearings were analysed (Figure $55 \mathrm{~B}-\mathrm{F}$ ). For the analysis shown, only the time between the second injection until 60 min post injection has been taken into account, as effects are strongly attenuated later on.

MK801-treated animals showed a hyperactivity phenotype compared to controls. Mice treated with Spironolactone displayed a tendency towards less distance travelled (Figure 55 B). Interestingly, mice treated both with MK801 and Spironolactone showed a non-significant tendency towards less distance travelled compared to mice injected with MK801 only.

When the total locomotor behaviour was analysed, the non-significant tendency observed in doubly-injected mice was not visible (Figure $55 \mathrm{C}$ ).

Of interest, mice injected with Spironolactone only spent less time being active (Figure 55.D), and also spent less time in the centre of the open field arena compared to a control group (Figure $55 \mathrm{E}$ ). As reported before, MK801injected mice displayed readily a higher activity than controls, and spent only a little amount of time in the centre. Double-treated mice, however, showed only a non-significant tendency towards less activity, and also spent only a very low percentage of the time in the centre of the test arena (Figure $55 \mathrm{E}$ ).

Last, I looked at rearings to assess curiosity. However, the analysis showed no significant measure compared to controls (Figure $55 \mathrm{~F}$ ).

Taken together, our results suggest that Spironolactone reduces spontaneous locomotor activity in the open field test as adding Spironolactone on top of a MK801 treatment may lead to slightly attenuated properties on mouse behavior. 
Results

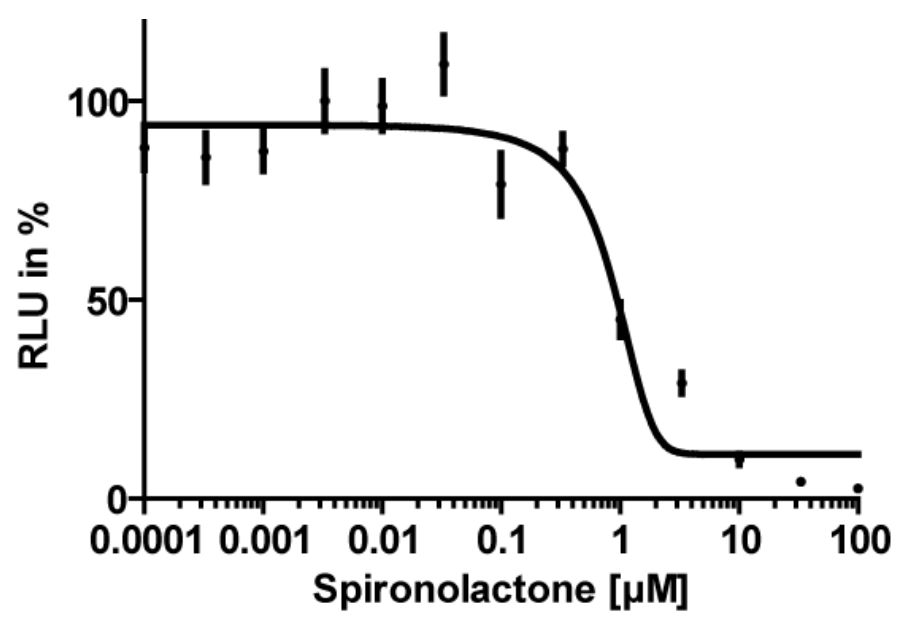

Figure 38: Dose Response of SpironolactoneSplit TEV assay in PC12 cells.

ERBB4 was fused to NTEV-tevS-GV-2xHA; PIK3R1 was fused to CTEV-2HA. ERBB4 dimerises after stimulation with 10.000 NRG1-Type I PC12 cells and recruits PIK3R1. Cells were treated with indicated concentrations of Spironolactone. Cells were harvested and analysed after $24 \mathrm{~h}, \mathrm{n}=6$, error bars represent standard error of the mean. 
A

Merge technical controls

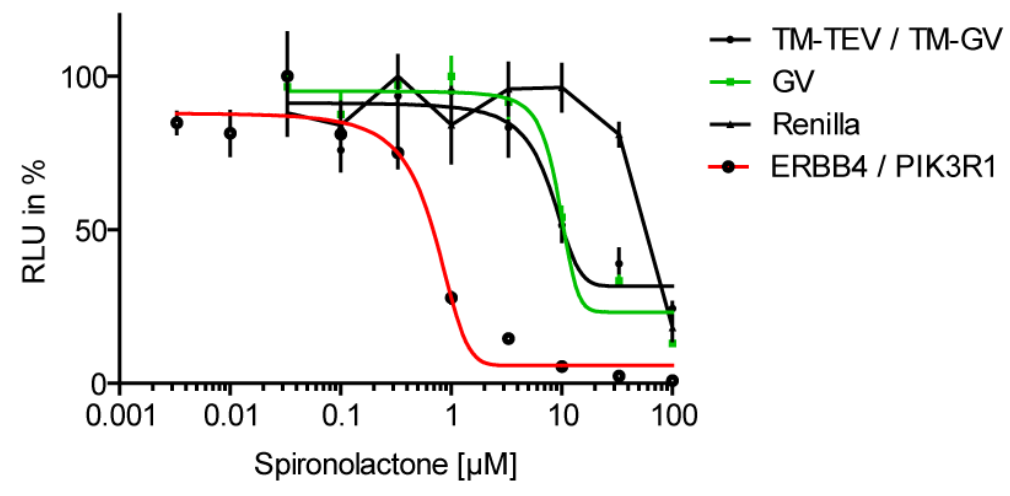

B
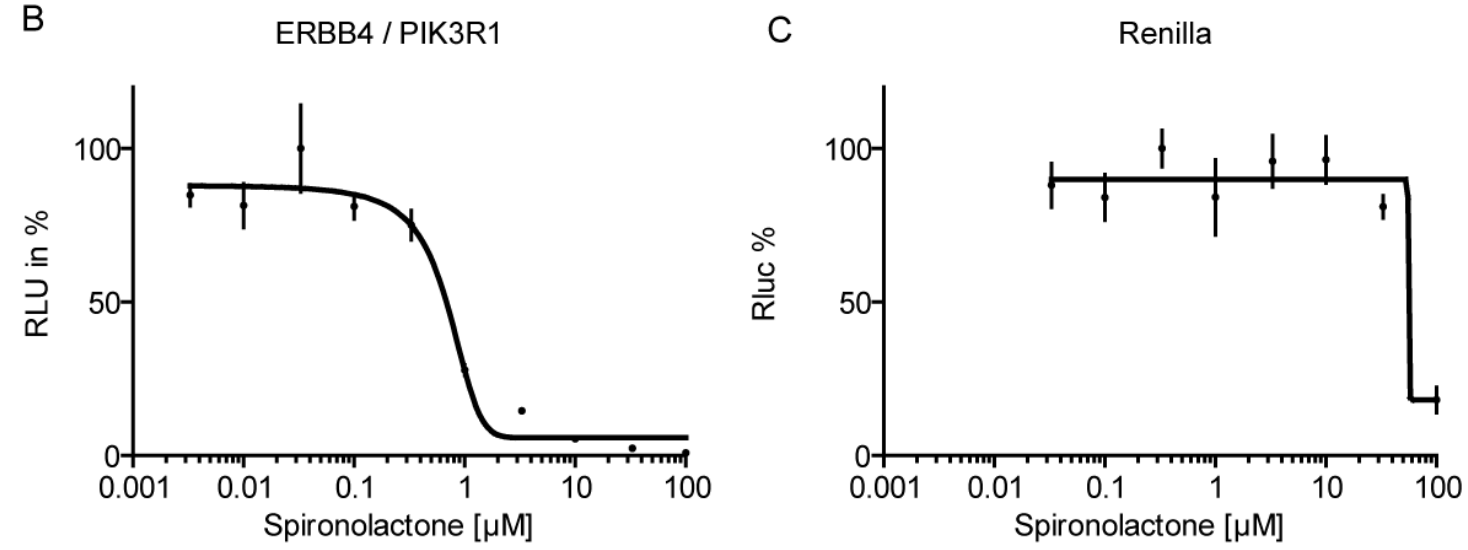

GV

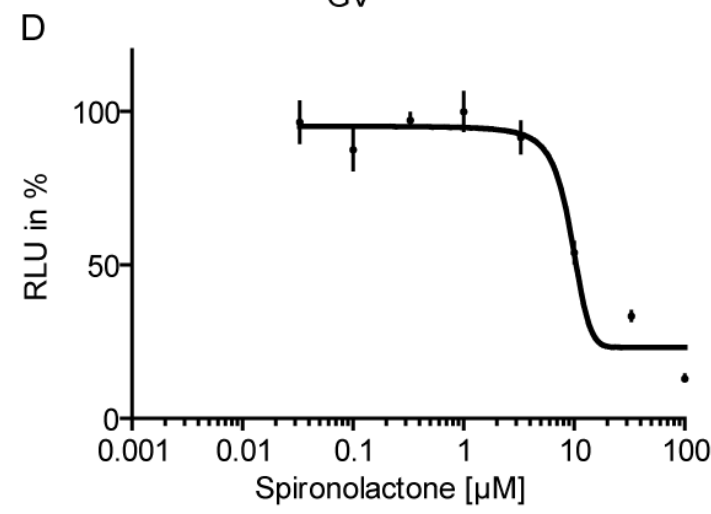

TM-TEV / TM-GV

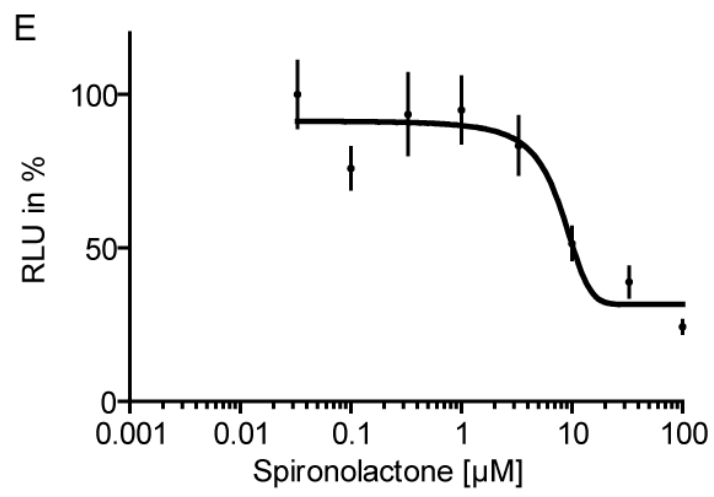

Figure 39: Technical controls

A) Merge of the results of the technical controls versus ERBB4/PIK3R1 (red).

Datasets shown from (B) to (E) were merged. B) ERBB4/PIK3R1. ERBB4 was fused to NTEV-tevS-GV-2xHA; PIK3R1 was fused to CTEV-2HA. Cells were stimulated with 10ng/nl EGFld. C) Renilla Luciferase assay Cells were transfected with 20ng/well TK-Rluc. D) GV assay in PC12 cells. Cells were transfected with 20ng/well CMV-GV, UAS-Fluc, and TK-Rluc. E) TM-TEV/TM-GV assay in PC12 cells. Cells were transfected with 20ng/well TM-TEV, TM-GV. 
A

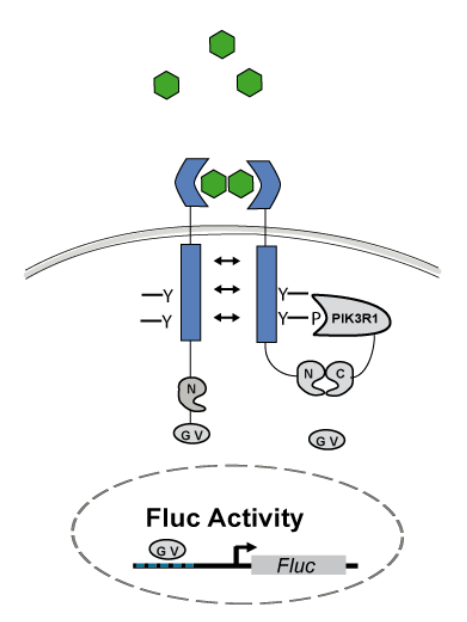

B

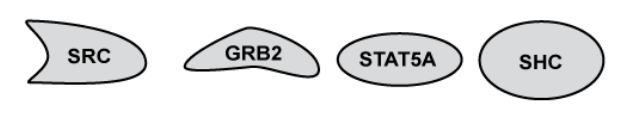

C

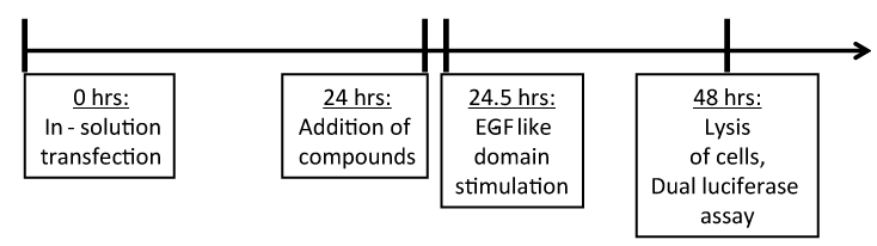

D

\begin{tabular}{|l|l|l|}
\hline RTK & Interaktor & Z'-factor \\
\hline ERBB4 & GRB2 & 0,3 \\
\hline & PIK3R1 & 0,6 \\
\hline & SHC1 & 0,2 \\
\hline & SRC & 0,2 \\
\hline & STAT5A & $-3,4$ \\
\hline
\end{tabular}

E

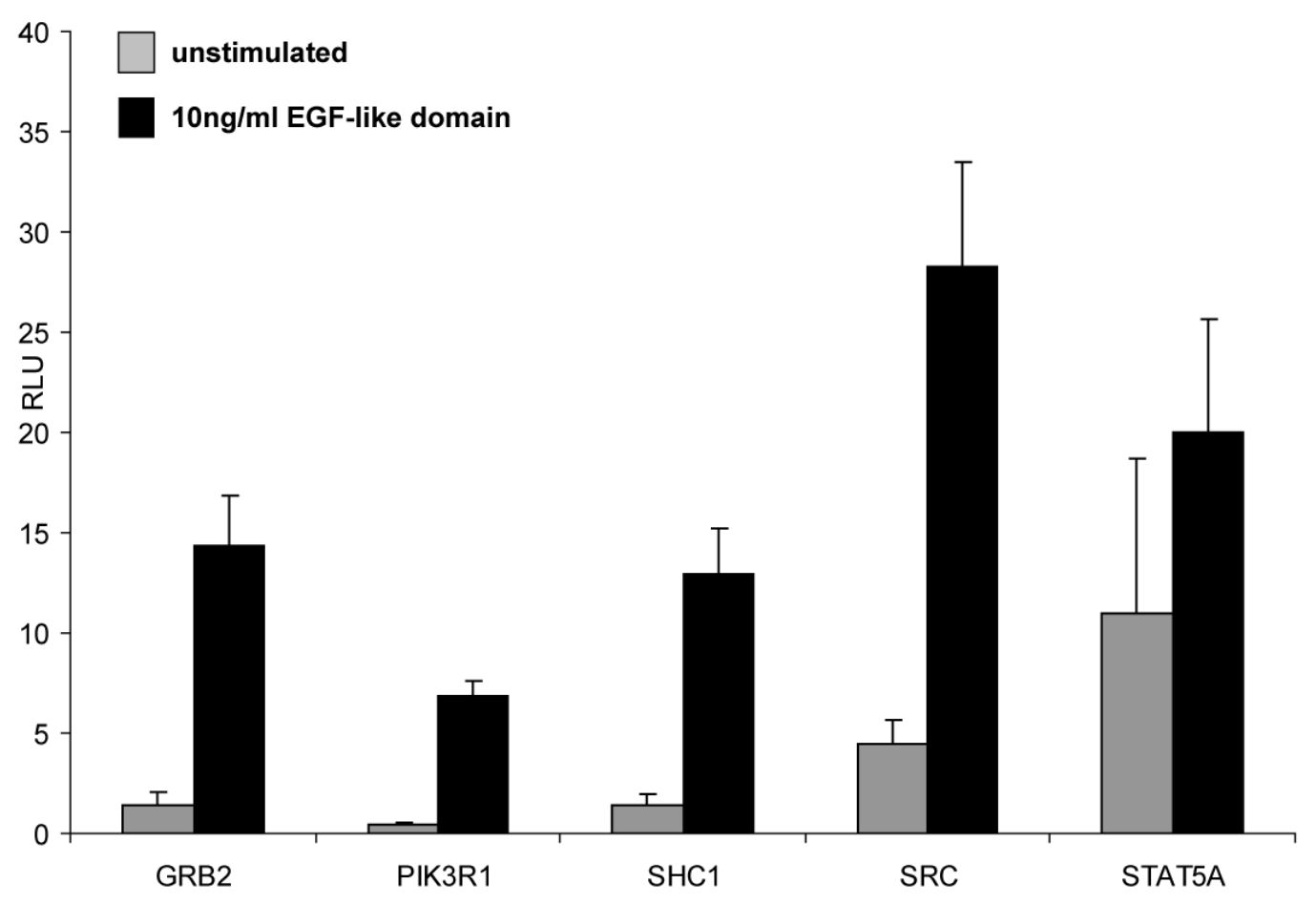

Figure 40: Vertical validation Spironolactone, overview

A) Schematic depiction of the ERBB4/adapter recruitment. B) Schematic drawings of the adapters used. C) Schematic drawings of experimental time course. Cells were transfected and after $24 \mathrm{~h}$ incubation time stimulated with $10 \mathrm{ng} / \mathrm{ml}$ EGFId. Cells were lysed and analysed $24 \mathrm{~h}$ after stimulation. D) Z'-factors obtained from the assays.

E) ERBB4/adapter recruitment. ERBB4 was fused to NTEV-tevS-GV-2xHA; adapters were fused to CTEV-2HA. Cells were stimulated with $10 \mathrm{ng} / \mathrm{ml}$ EGFld. 
A Merge Adapters

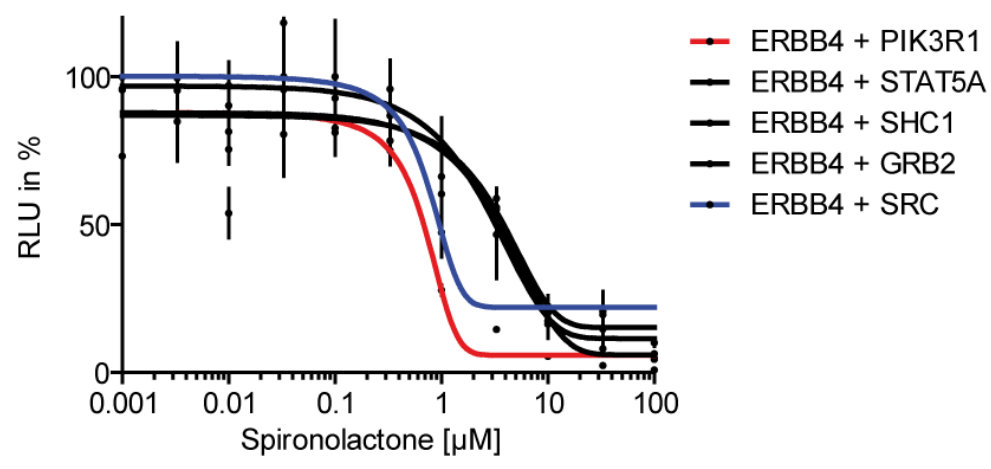

B

ERBB4 / STAT5A

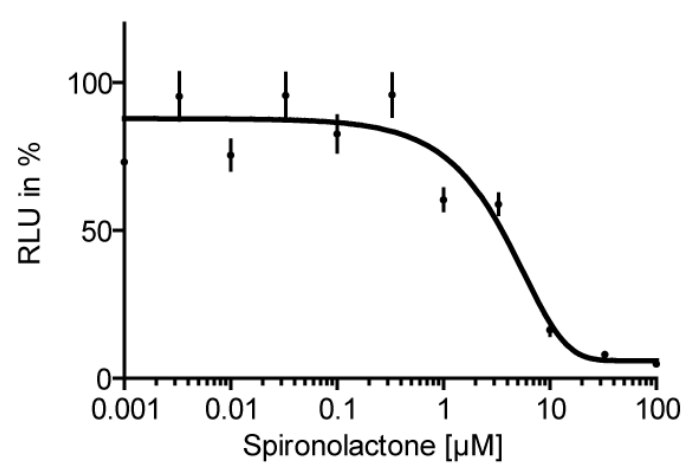

D

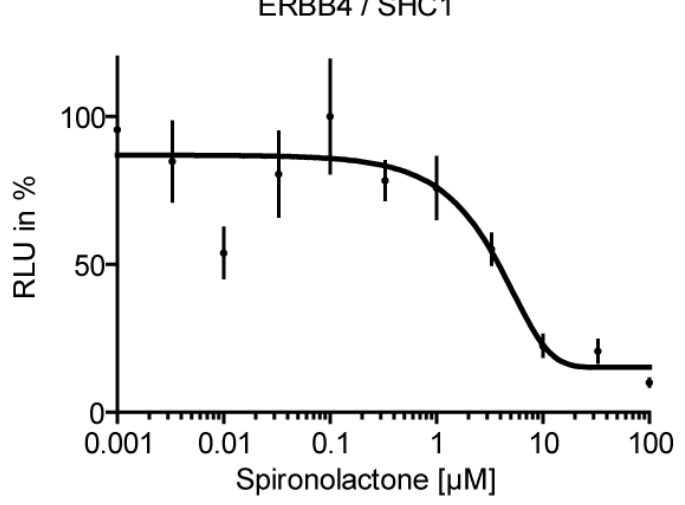

C

ERBB4 / GRB2

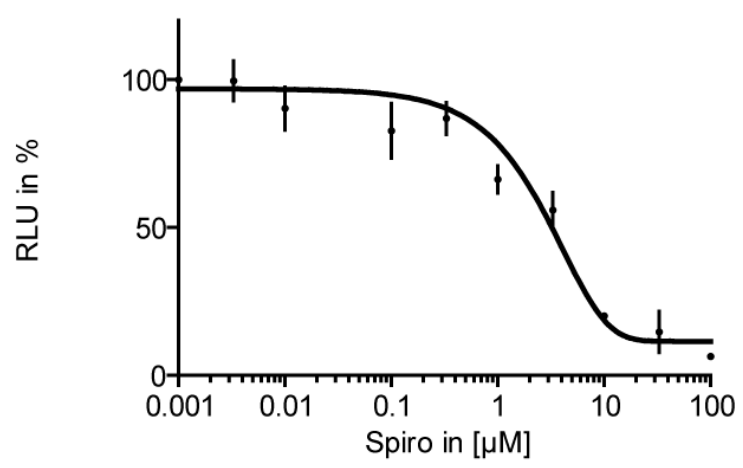

E

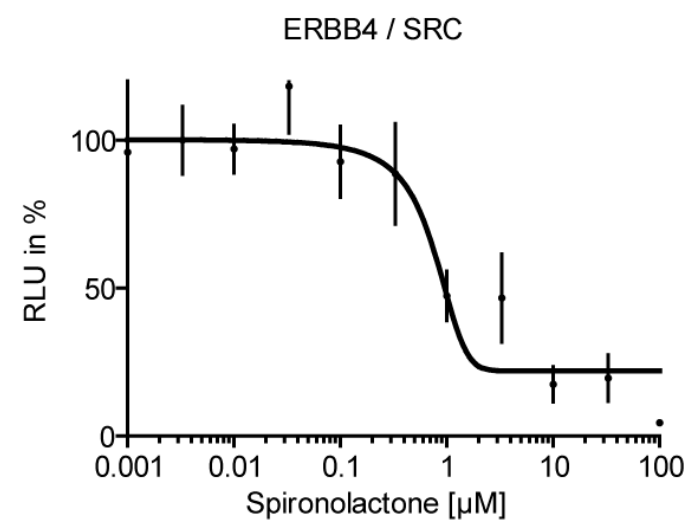

Figure 41: Vertical validation Spironolactone ERBB4/adapter recruitment

A) Comparison of ERBB4/adapter recruitment assays with ERBB4-PIK3R1 recruitment (red). ERBB/adapter recruitment for SHC1, GRB2, and STAT5a are less efficiently inhibited as compared to ERBB4/PIK3R1 recruitment. ERBB4/SHC1 is intermediate. B-E) ERBB4/adapter recruitment. ERBB4 was fused to NTEV-tevS-GV-2xHA; adapters were fused to CTEV-2HA. PC12 cells were stimulated with $10 \mathrm{ng} / \mathrm{ml}$ EGFld. 
A

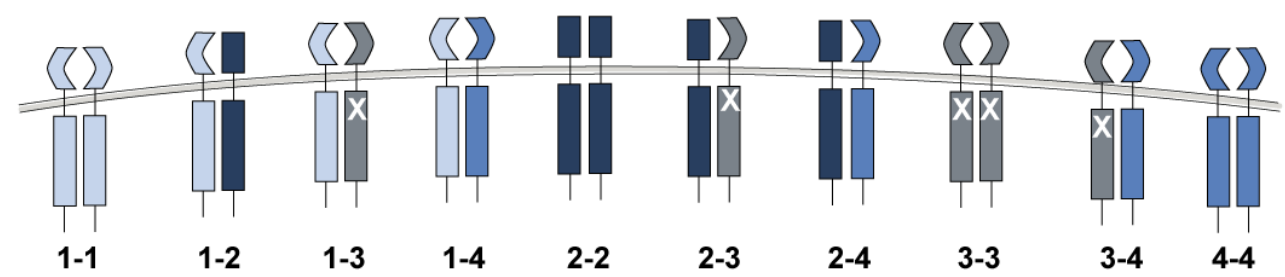

B

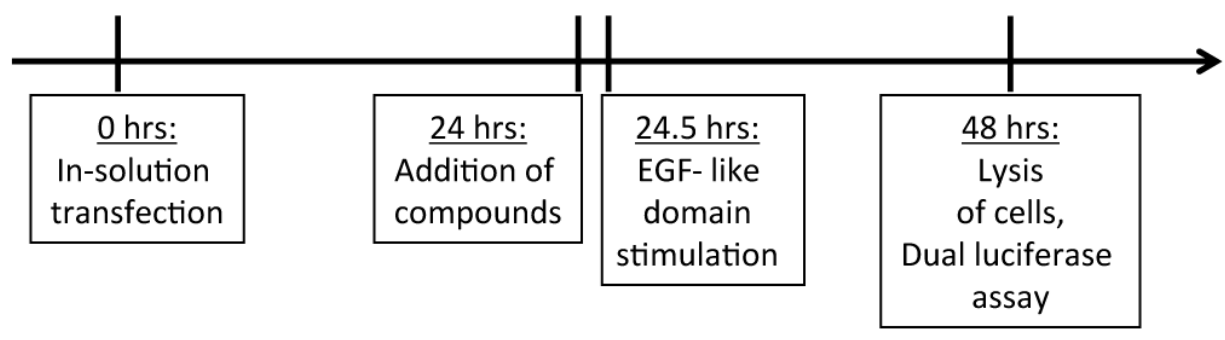

C

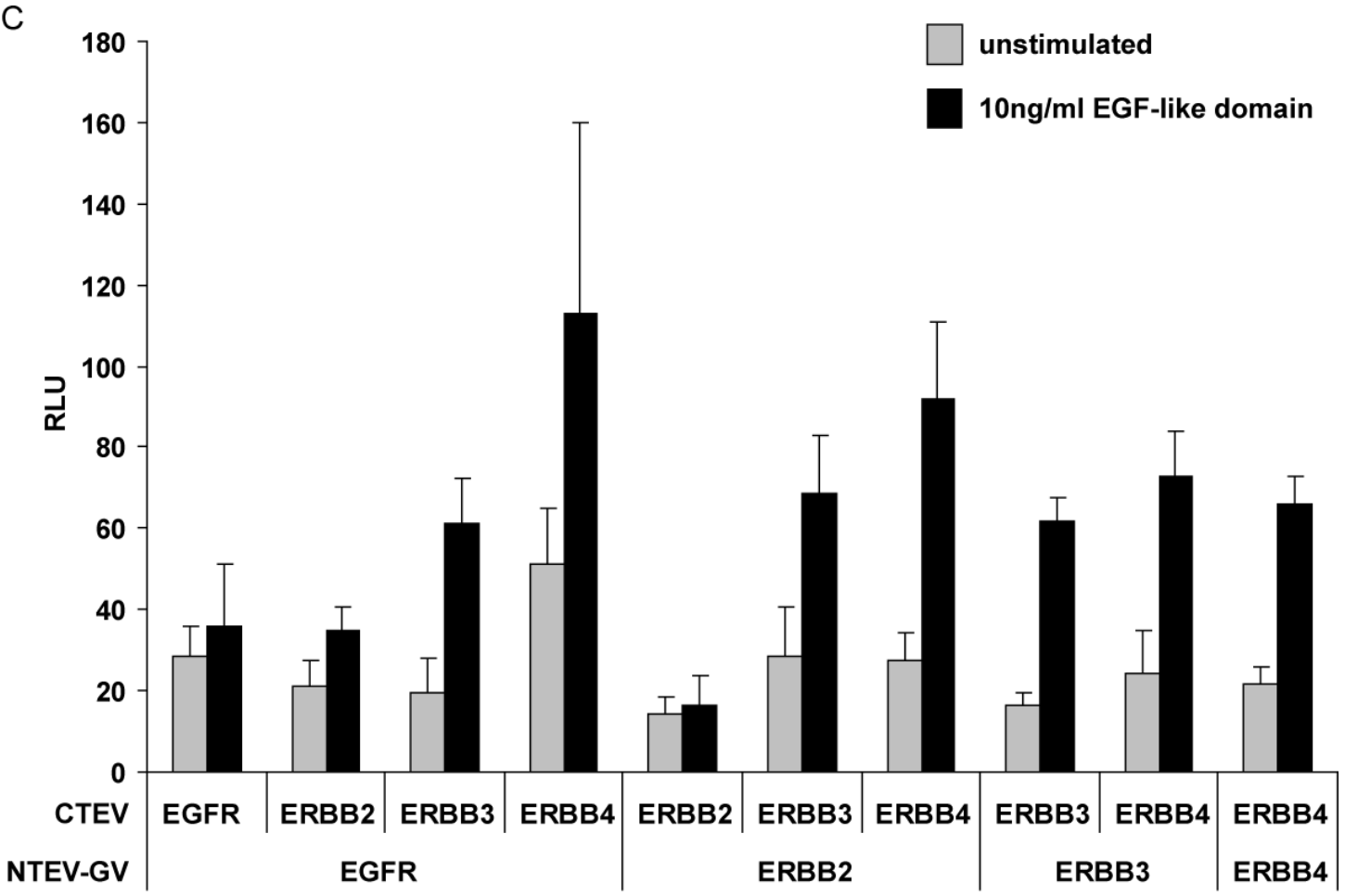

Figure 42: Homo and heterodimerisations of ERBB receptors

A) Schematic drawings of all possible ERBB homo and heterodimer combinations. Note, that ERBB1 (EGFR) is activated by EGF, and not by NRG1-typel, and that ERBB2 lacks a NRG1-binding site whereas ERBB3 lacks intrinsic kinase activity. ERBB4 binds to NRG1 as homodimer and is able to cross-phosphorylate itself. B) Schematic drawings of experimental time course. Cells were transfected and after $24 \mathrm{~h}$ incubation time stimulated with $10 \mathrm{ng} / \mathrm{ml}$ EGFld. Cells were lysed and analysed $24 \mathrm{~h}$ after stimulation. C) ERBB homoand hetero-dimerisation. ERBBx/ERBBy split TEV assay in PC12 cells. ERBBx was fused to NTEV-tevS-GV-2xHA; ERBBy was fused to CTEV-2HA. Cells were stimulated with $10 \mathrm{ng} / \mathrm{ml}$ EGFId. 
A

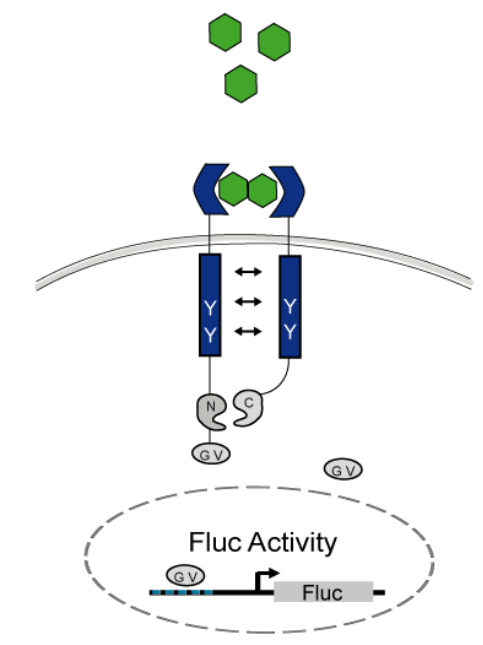

C

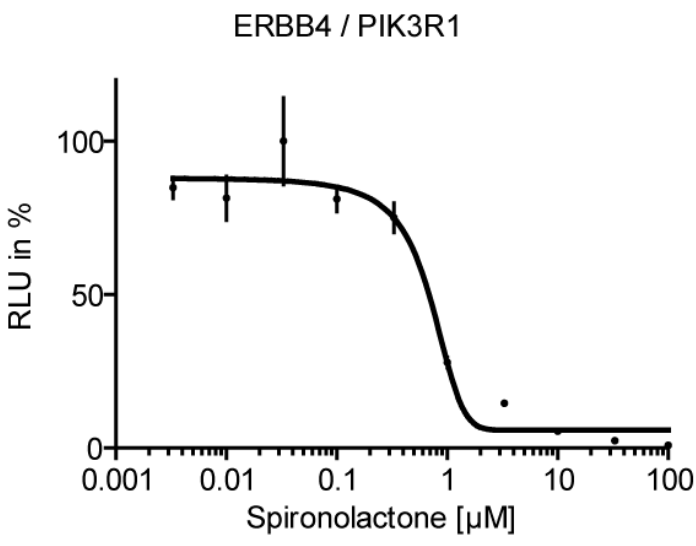

B

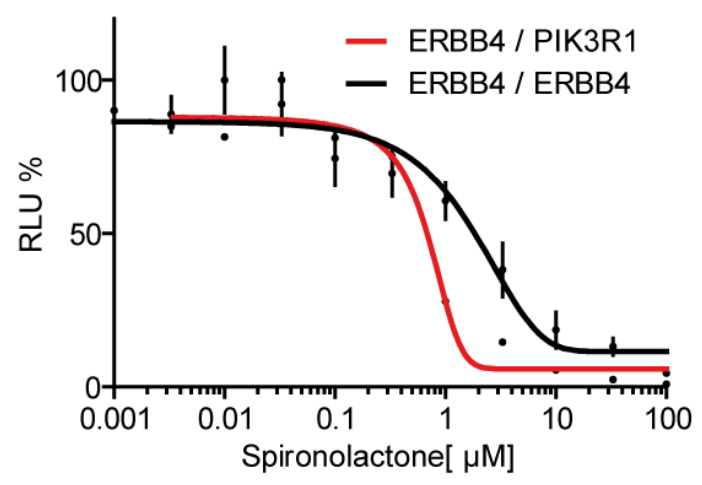

D

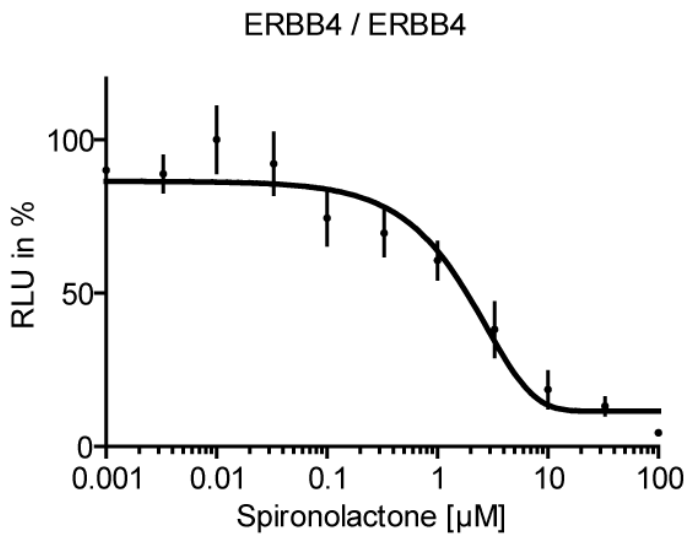

Figure 43: ERBB4 homodimerisation

A) Schematic depiction of ERBB4 homodimerisation. B) Comparison of ERBB4 homodomerisation with ERBB4/PIK3R1 interaction. C) ERBB4/PIK3R1. D) ERBB4/ERBB4. ERBB4 was fused to NTEV-tevS-GV-2xHA; PIK3R1 and ERBB4 were fused to CTEV-2HA. PC12 cells were stimulated with $10 \mathrm{ng} / \mathrm{ml}$ EGFld. 
Results

A

MERGE ERBB Panel

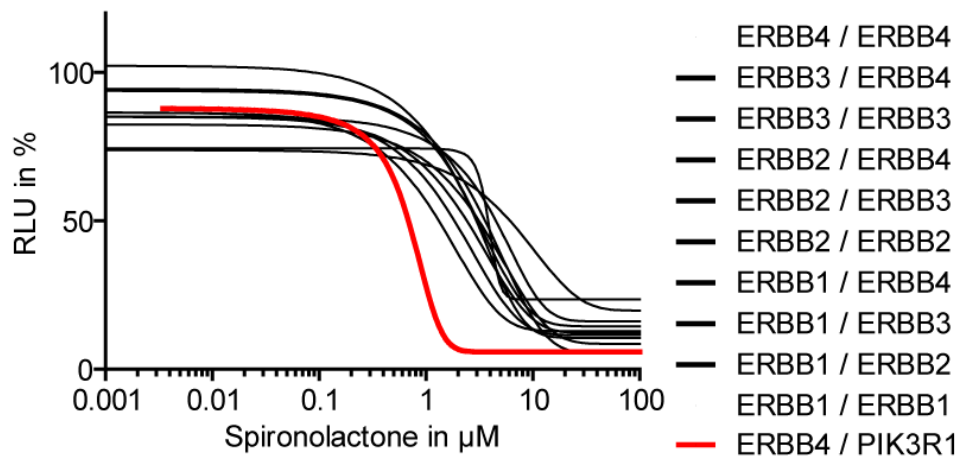

B

ERBB1 / ERBB1

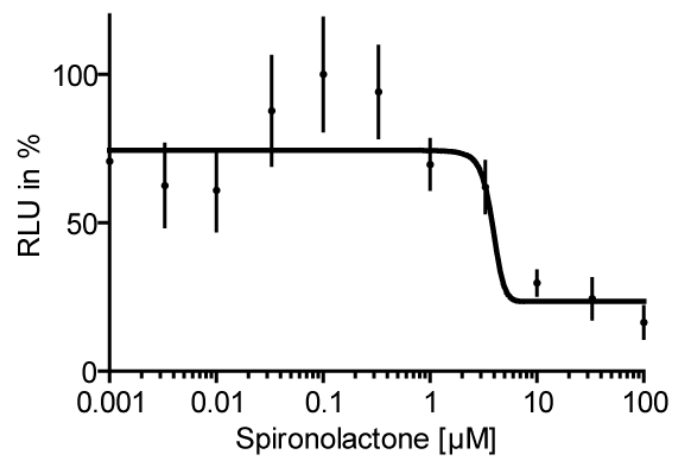

C

ERBB1 / ERBB2

D

ERBB1 / ERBB3

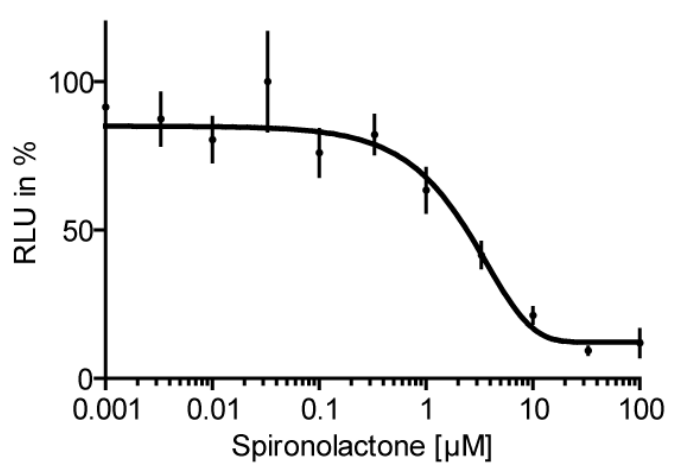

E

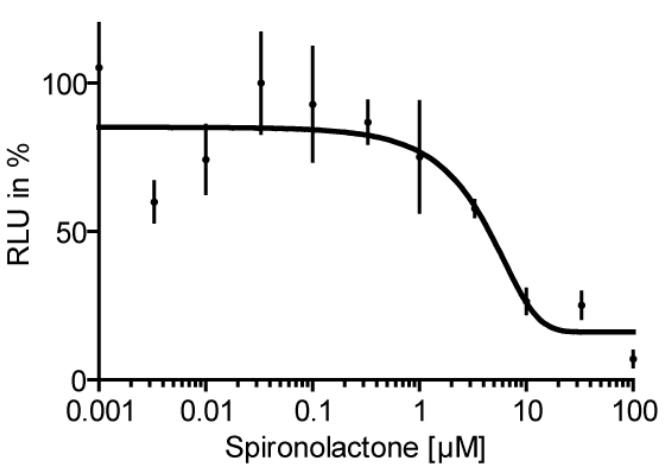

ERBB1 / ERBB4

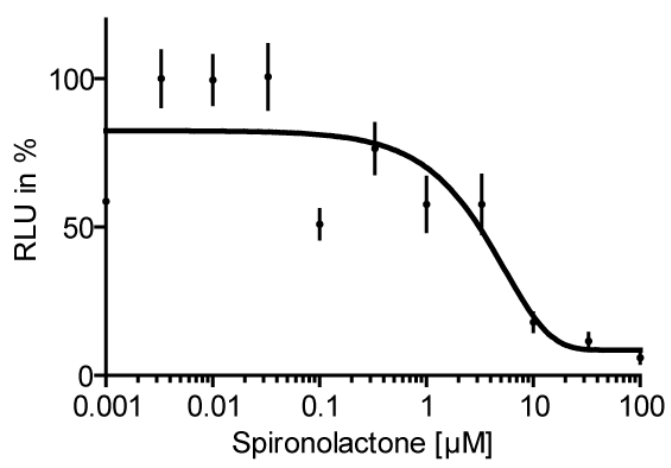


Results

$\mathrm{F}$

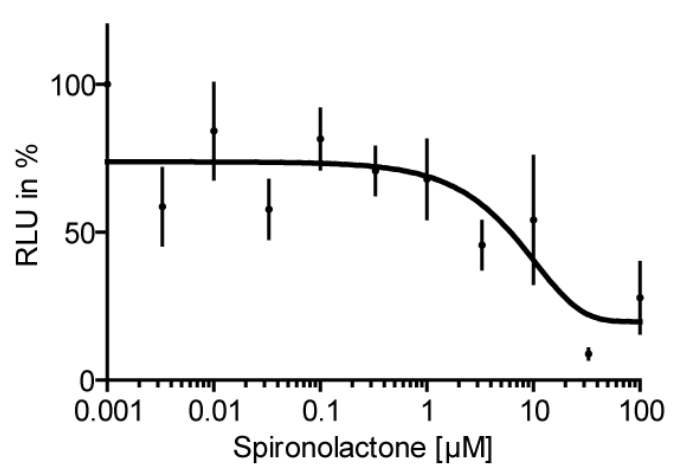

$\mathrm{H}$

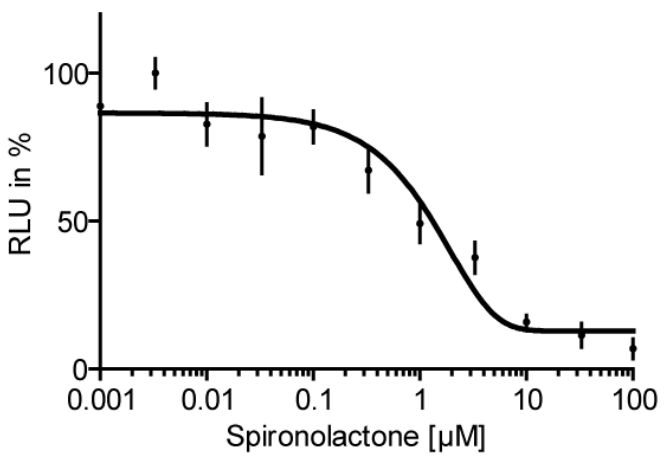

$J$

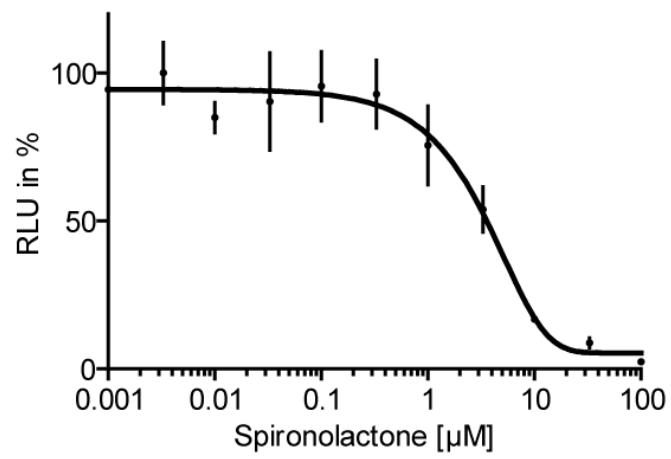

G

ERBB2 / ERBB3

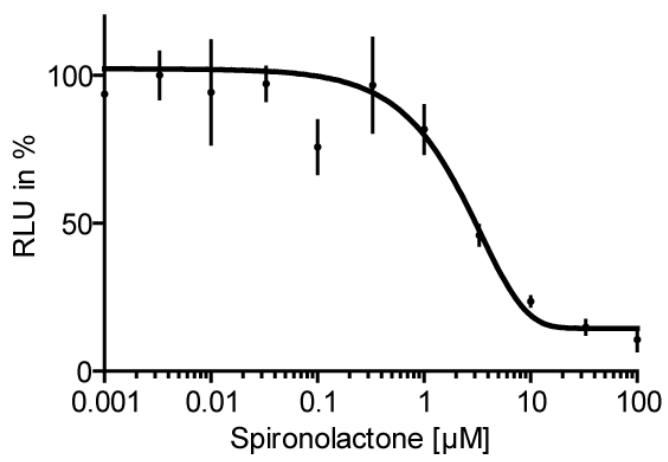

I

ERBB3 / ERBB3

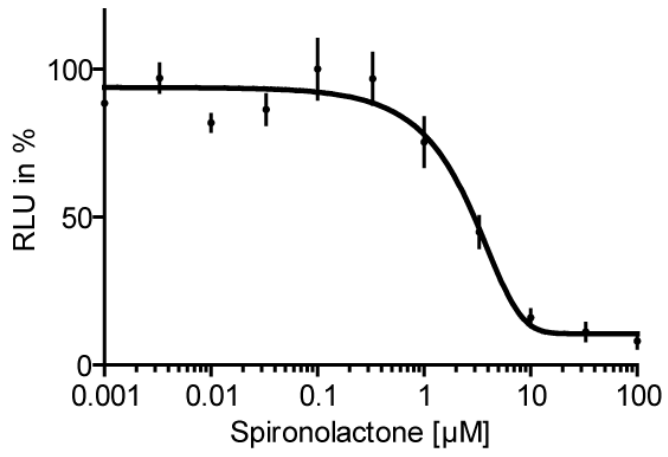

$\mathrm{K}$

ERBB4 / ERBB4

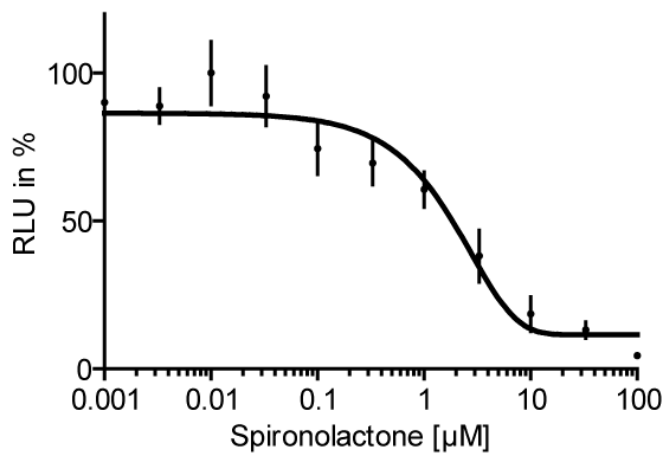

Figure 44: ERBB homo and heterodimerisation

A) Comparison of ERBB homo and heterodimerisation assays with ERBB4-PIK3R1 recruitment. ERBB homo/heterodimerisations are less efficiently inhibited as compared to ERBB4-PIK3R1 recruitment. B-K) ERBB homo and heterodimerisations. ERBBx was fused to NTEV-tevS-GV-2xHA; ERBBy was fused to CTEV-2HA. PC12 cells were stimulated with $10 \mathrm{ng} / \mathrm{ml}$ EGFId. 

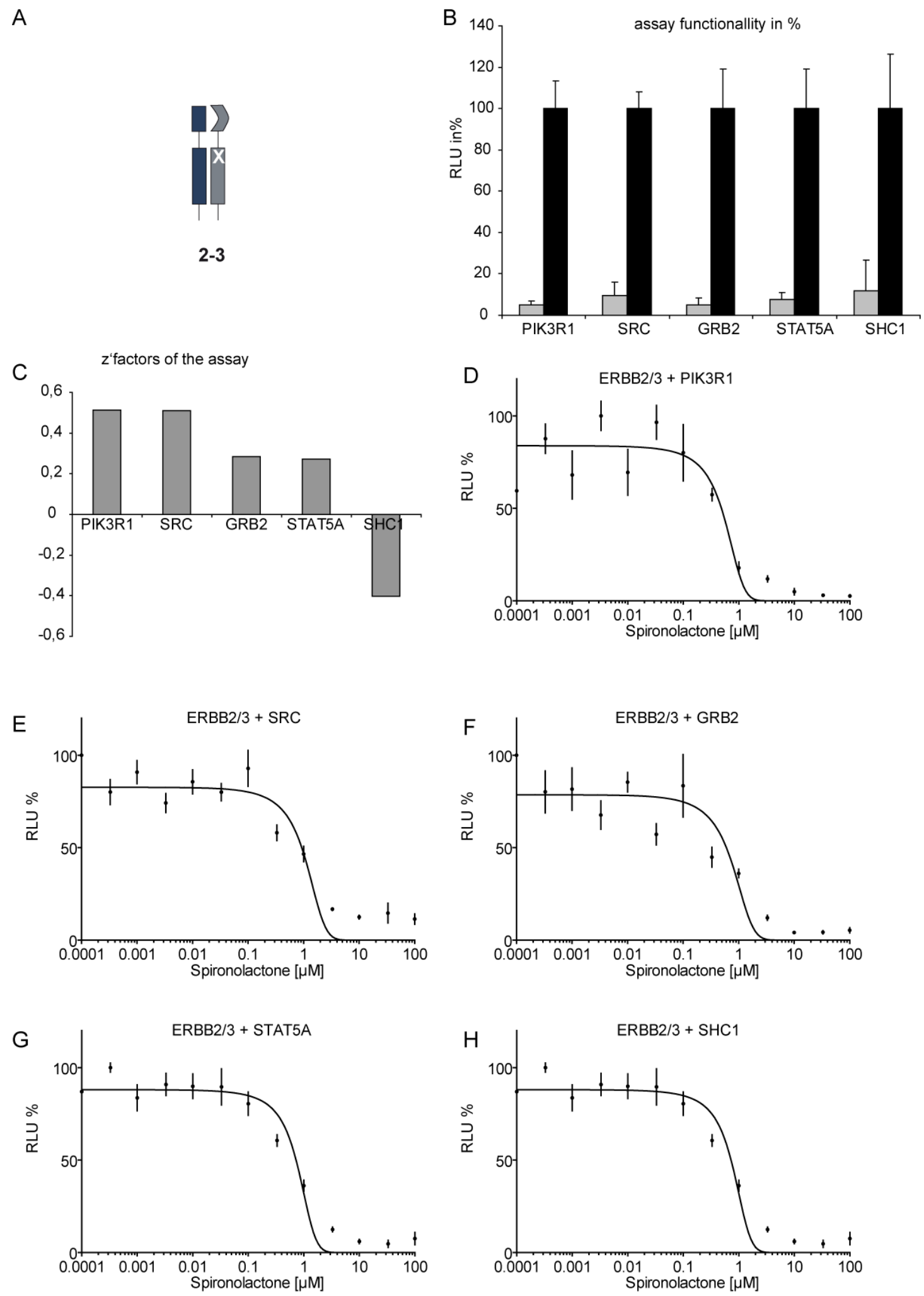

Figure 45: Horizontal validation ERBB2/3 and adapters

A) Schematic representation ERBB2/ERBB3 heterodimerisation. B) Assay performance. ERBB2/ERBB3 heterodimerise after stimulation with $10 \mathrm{ng} / \mathrm{ml}$ EGFld and recruit adapters as indicated. C) Comparison of the $z$ '-factors obtained from the assays. D-H) ERBB2/ERBB3/various adapters. ERBB2 was fused to a V5 tag, ERBB3 was fused to NTEV-tevS-GV-2xHA; adapters were fused to CTEV-2HA. PC12 cells were stimulated with $10 \mathrm{ng} / \mathrm{ml}$ EGFId. 
A

2-4
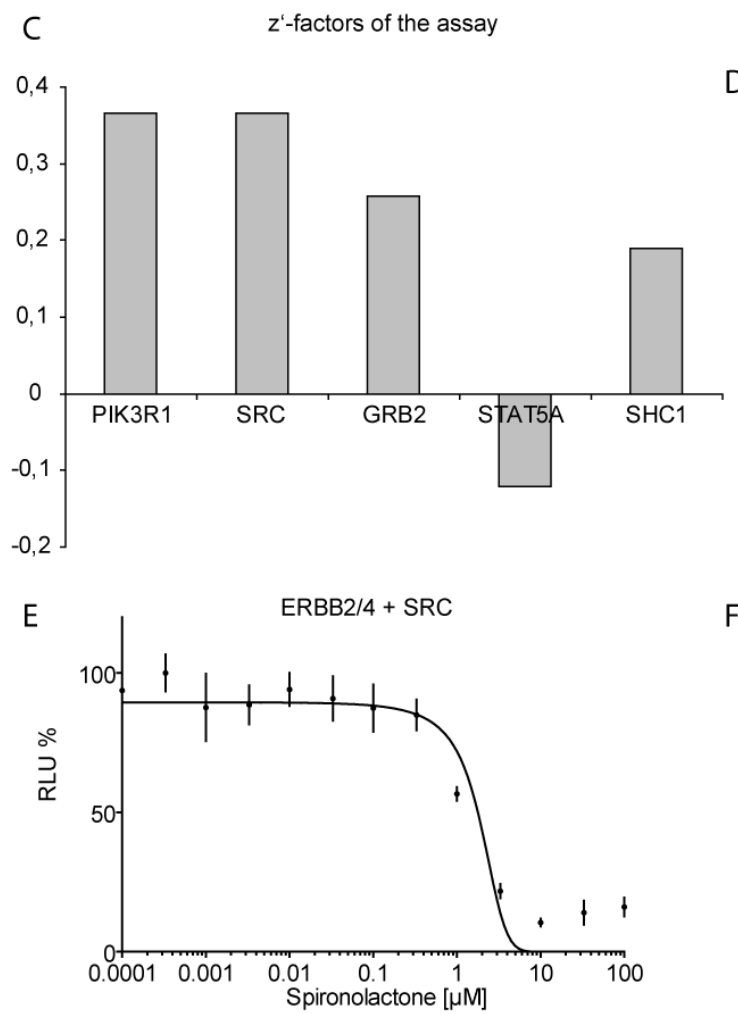

G

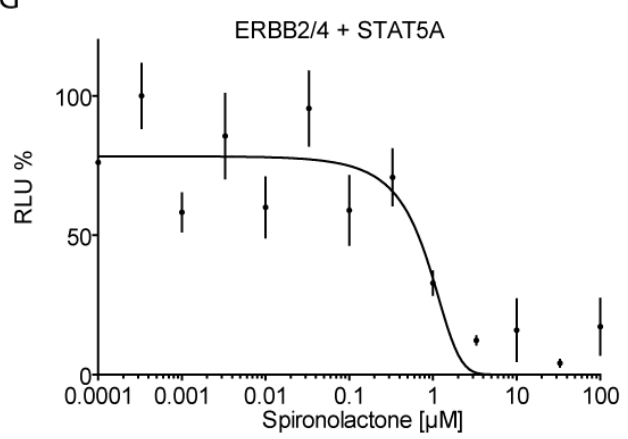

B

$\mathrm{F}$

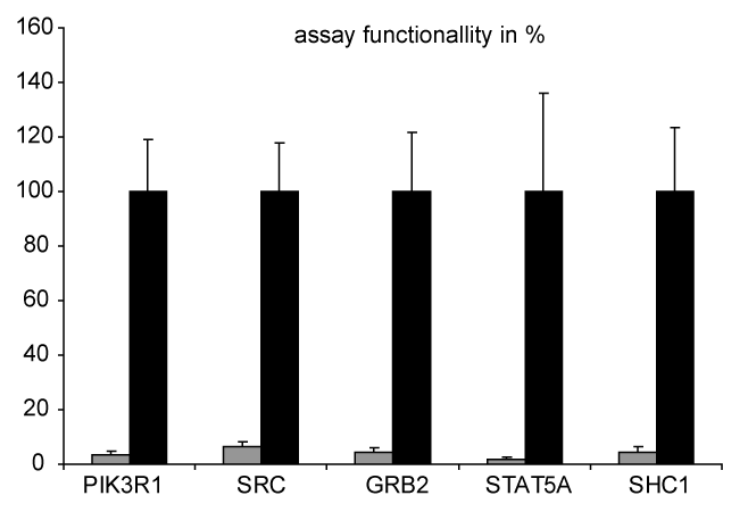

D
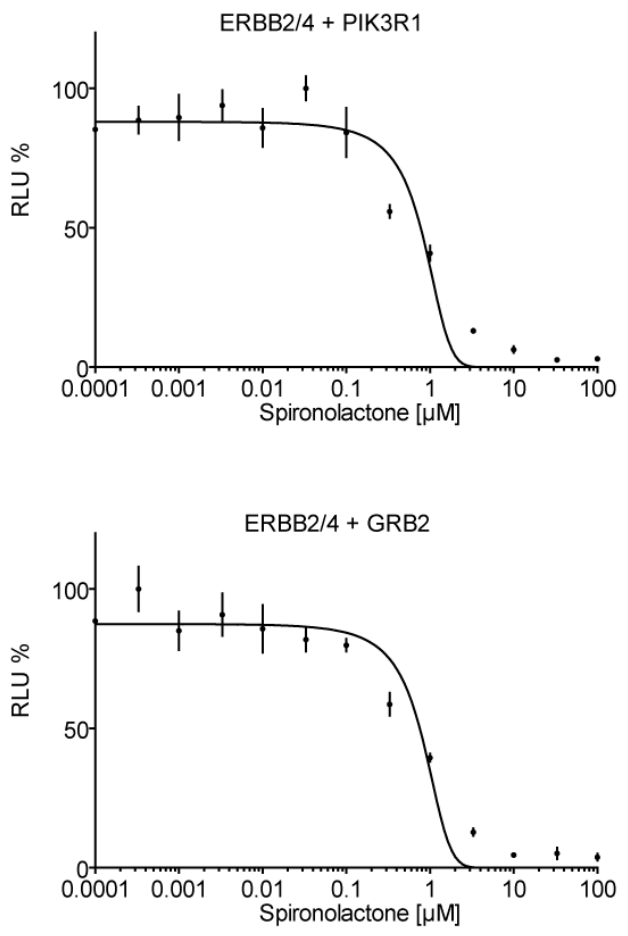

$\mathrm{H}$

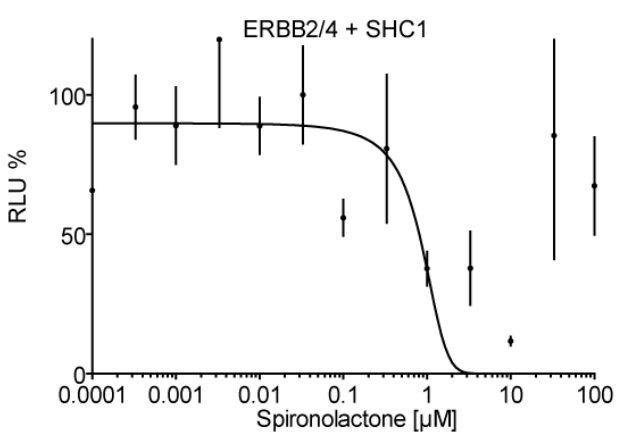

Figure 46: Horizontal validation ERBB2/ERBB4 and adapters

A) Schematic representation ERBB2/ERBB4 heterodimerisation. B) Assay performance. ERBB2/ERBB4 heterodimerise after stimulation with $10 \mathrm{ng} / \mathrm{ml}$ EGFld and recruit adapters as indicated. C) Comparison of the $z$ '-factors obtained from the assays.D-H) ERBB2/ERBB4/various adapters. ERBB2 was fused to a V5 tag, ERBB4 was fused to NTEV-tevS-GV-2xHA; adapters were fused to CTEV-2HA. PC12 cells were stimulated with $10 \mathrm{ng} / \mathrm{ml}$ EGFId. 
Results
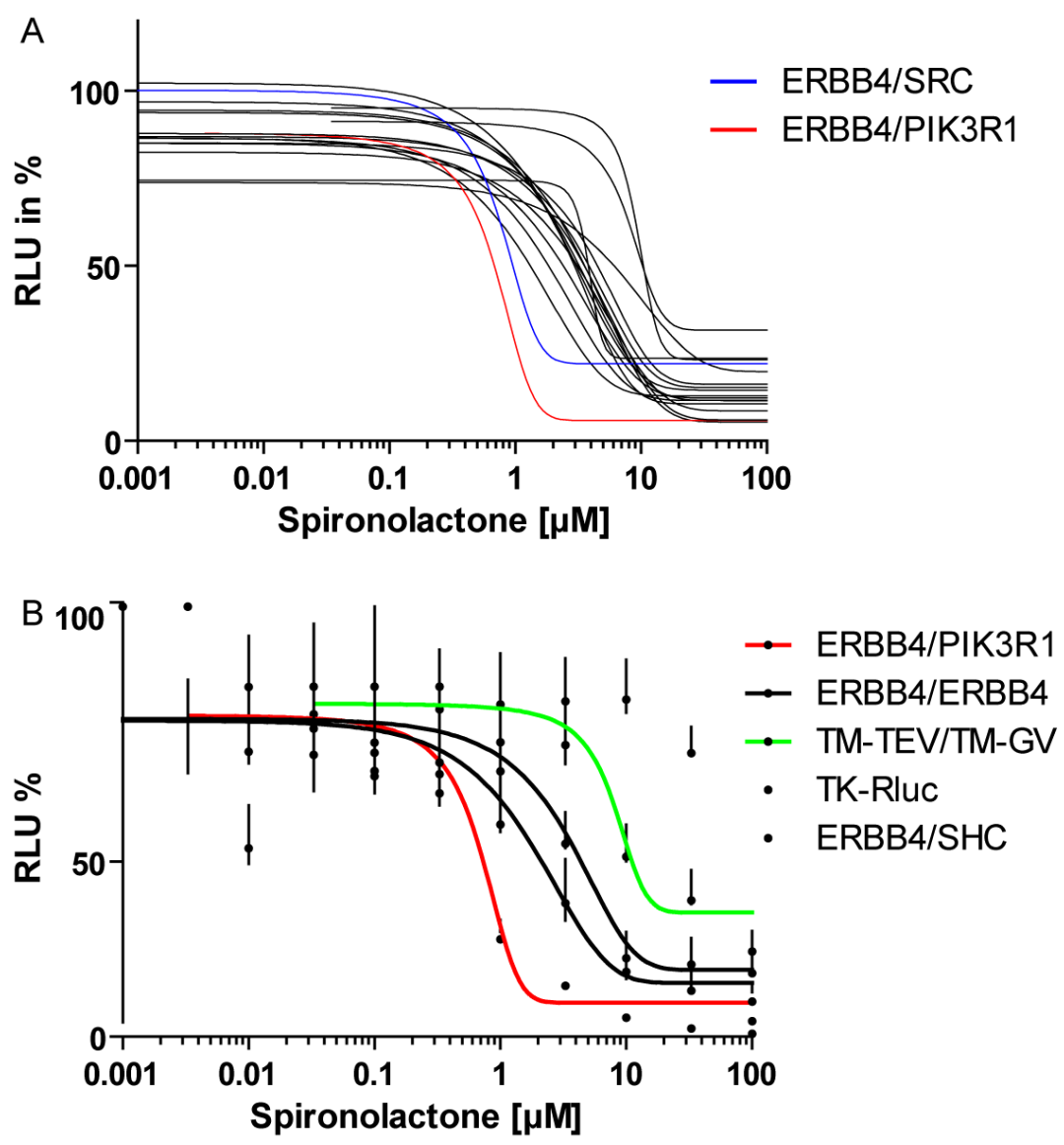

Figure 47: Data compilation of horizontal and vertical validation assays with Spironolactone

A) Detailed comparison of the datasets of the technical, vertical and horizontal validation.

B) Compilation of selected analyses of the technical, vertical, and horizontal validation. 


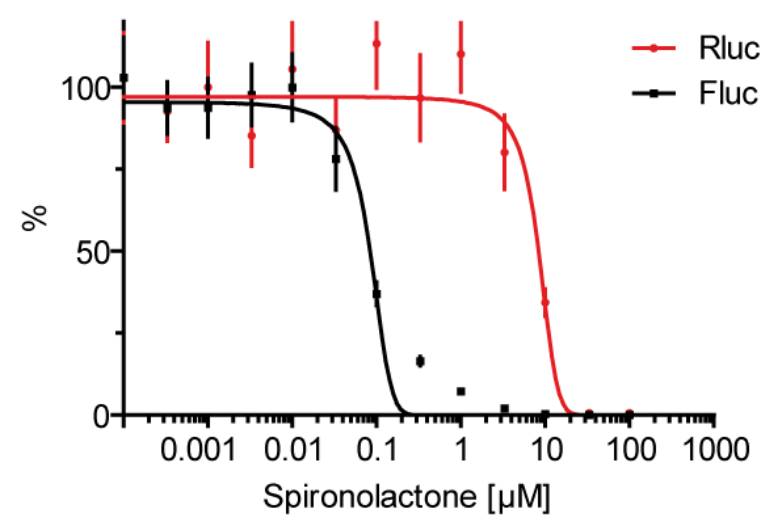<smiles>CC(=O)S[C@H]1CC2=CC(=O)CCC2(C)C2CCC3(C)C(CCC34CCC(=O)O4)C21</smiles>

B Canrenone

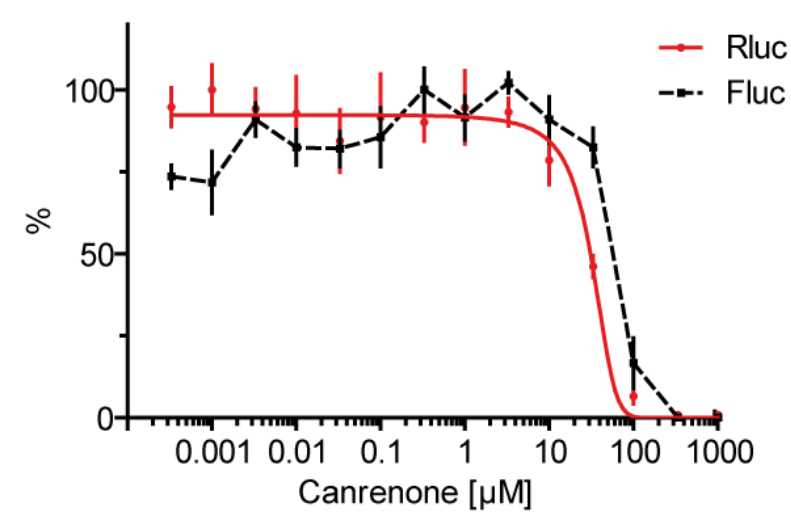<smiles>CC12CCC(=O)C=C1C=CC1C2CCC2(C)C1CCC21CCC(=O)O1</smiles>

C Eplerenone

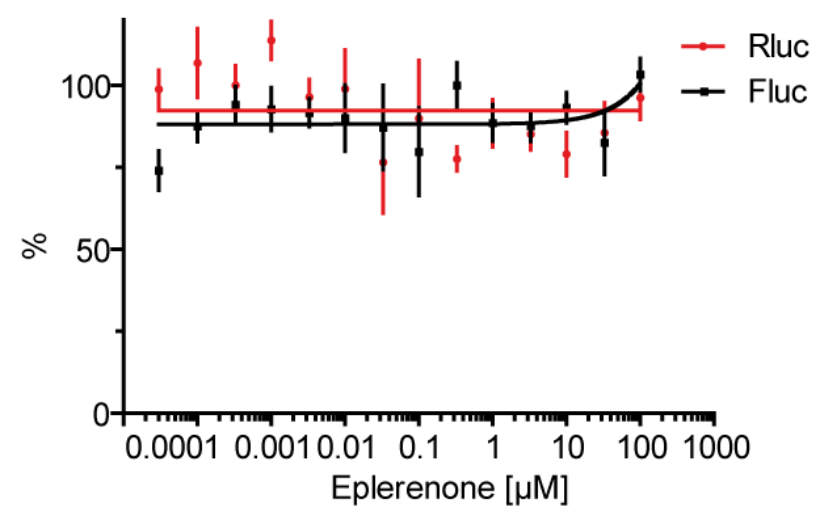<smiles>COC(=O)[C@H]1CC2=CC(=O)CCC2(C)[C@@]2(C)C[C@@H]3CC4(C)C(CC[C@]45CCC(=O)O5)[C@]3(C)C12</smiles>

Figure 48: Comparison Spironolactone, Canrenone, Eplerenone

A) Spironolactone, exerts a strong dose-response dependent inhibitory effect on the recruitment of PIK3R1 to ERBB4 (Fluc and Rluc signal). A') Molecular structure (skeletal formula) of Spironolactone. B) Canrenone (metabolite of Spironolactone) exerts no doseresponse dependent effect on the recruitment of PIK3R1 to ERBB4. B') Molecular structure (skeletal formula) of Canrenone. C) Eplerenone $\left(2^{\text {nd }}\right.$ generation substance of Spironolactone) exerts no dose-response dependent effect on the recruitment of PIK3R1 to ERBB4. C') Molecular structure (skeletal formula) of Eplerenone. All assays are ERBB4/PIK3R1 dimerisation assays in PC12 cells, stimulated with10ng/ml EGFId. 
A

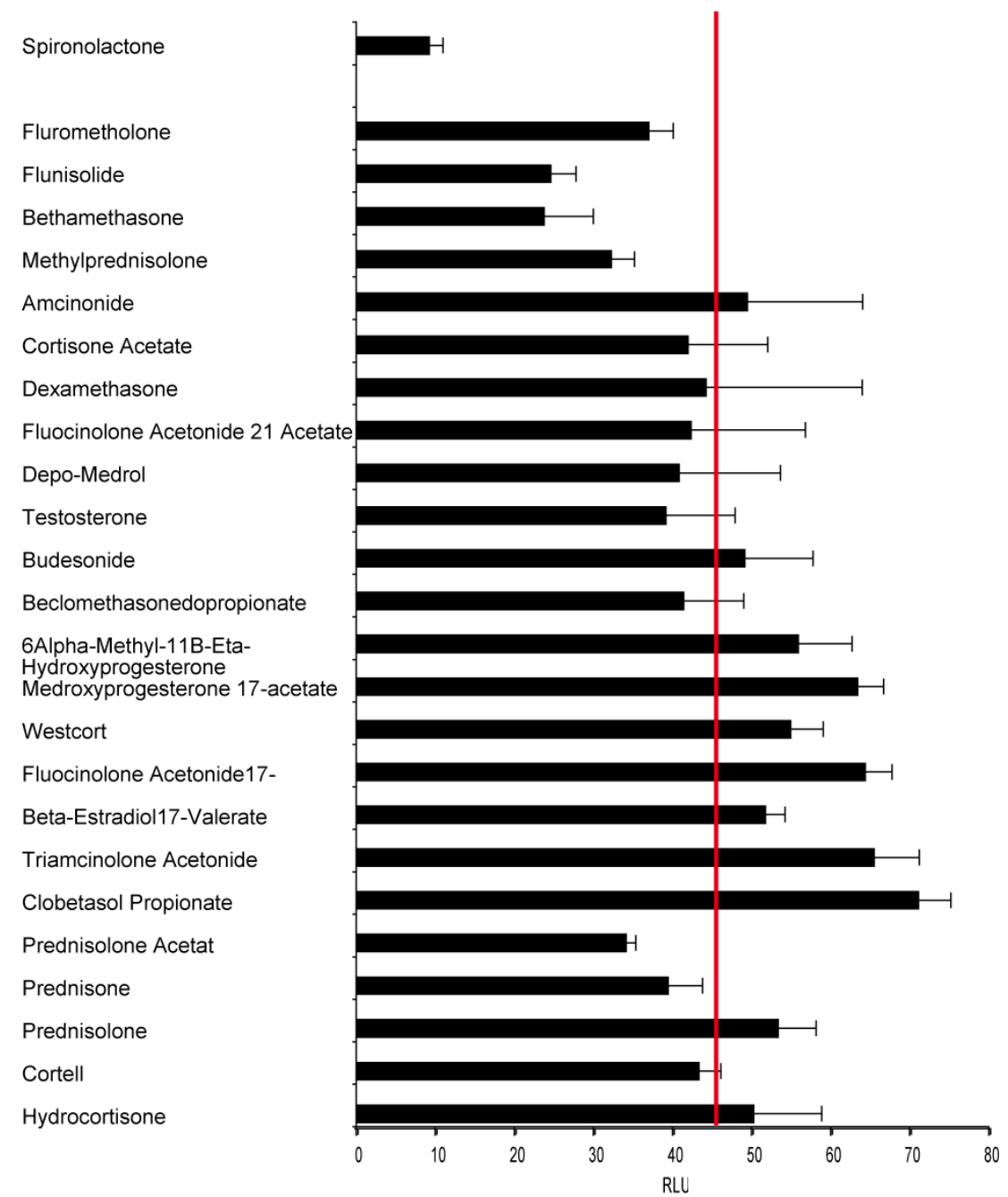<smiles>CC(=O)S[C@H]1CC2=CC(=O)CCC2(C)C2CCC3(C)C(CCC34CCC(=O)O4)C21</smiles>

Spironolactone

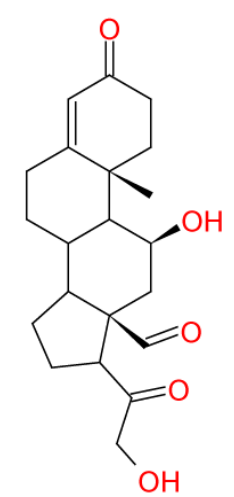

Aldosterone

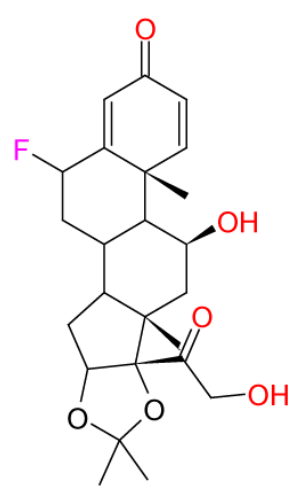

Flunisolide

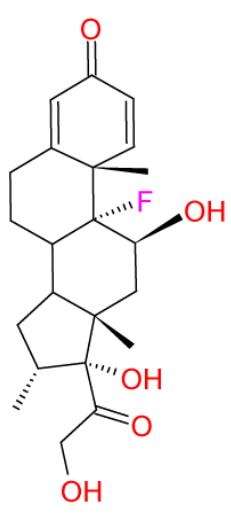

Betamethasone

Figure 49: Analysis of substances structurally related to Spironolactone

A) Selected results from the NCC201/NCC003 screen with sterol-related substances. The 25 compounds that show the closest structural relationship to Spironolacton were analysed for inhibition of an ERBB4/PIK3R1 co-culture assay (Compounds were suggested by pupchem.org.as related compounds) The red line indicates the mean of the combined effects (RLU 46.4; 100\%). Spironolactone was the most efficient substance in inhibiting the assay (RLU 9.3; 20\%). B) Molecular structures of Spironolactone, Aldosterone, Flunisolide and Betamethasone. Flunisolide and Betamethasone show an inhibitory effect in the screen without reaching the detection limit. 
Results
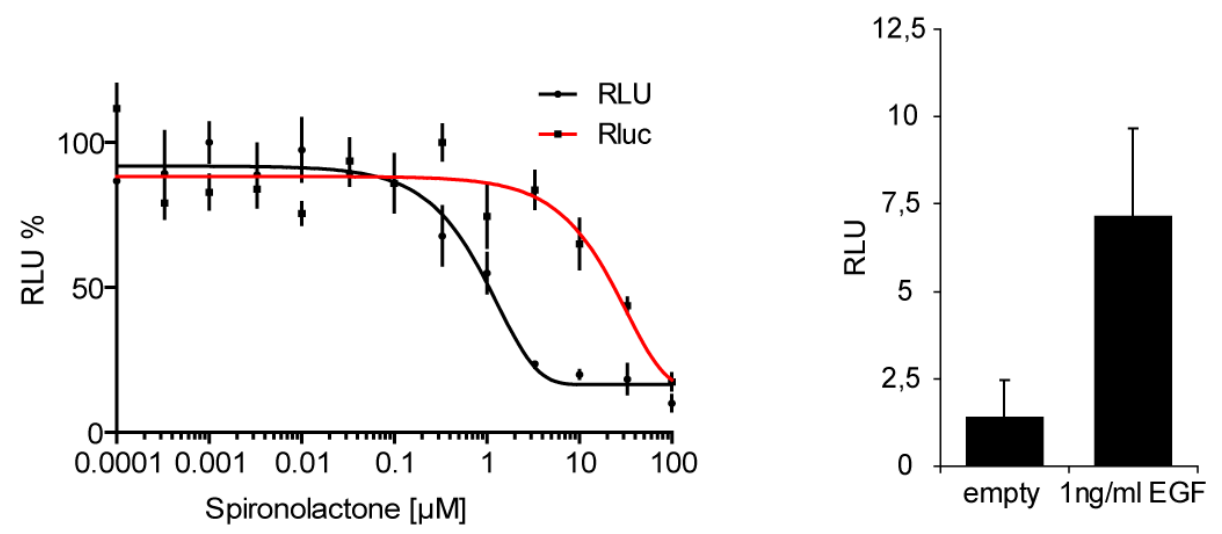

Figure 50: Effect of Spironolactone on ERBB1/1 dimerisation induced by EGF

ERBB1 was fused to NTEV-tevS-GV-2xHA; ERBB1 was fused to CTEV-2HA. PC12 cells were stimulated with $1 \mathrm{ng} / \mathrm{ml}$ EGF.
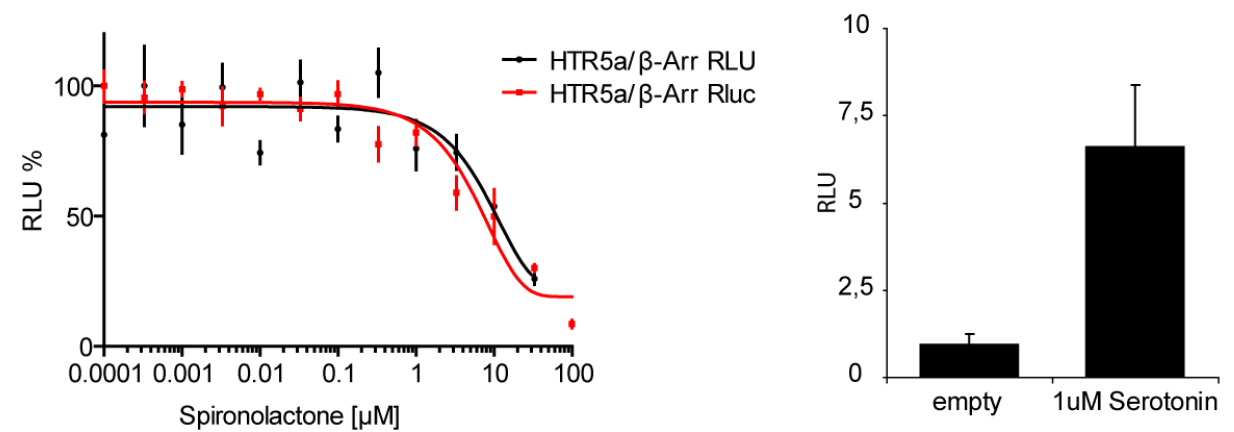

Figure 51: Effect of Spironolactone on stimulated HTR5a/ $\beta$-arrestin2

HTR5A was fused to NTEV-tevS-GV-2xHA; / $\beta$-arrestin2 $\Delta$ (amino acids 1-382 of ARRB2) was fused to CTEV-2HA. PC12 cells were stimulated with $1 \mu \mathrm{M}$ Serotonin.
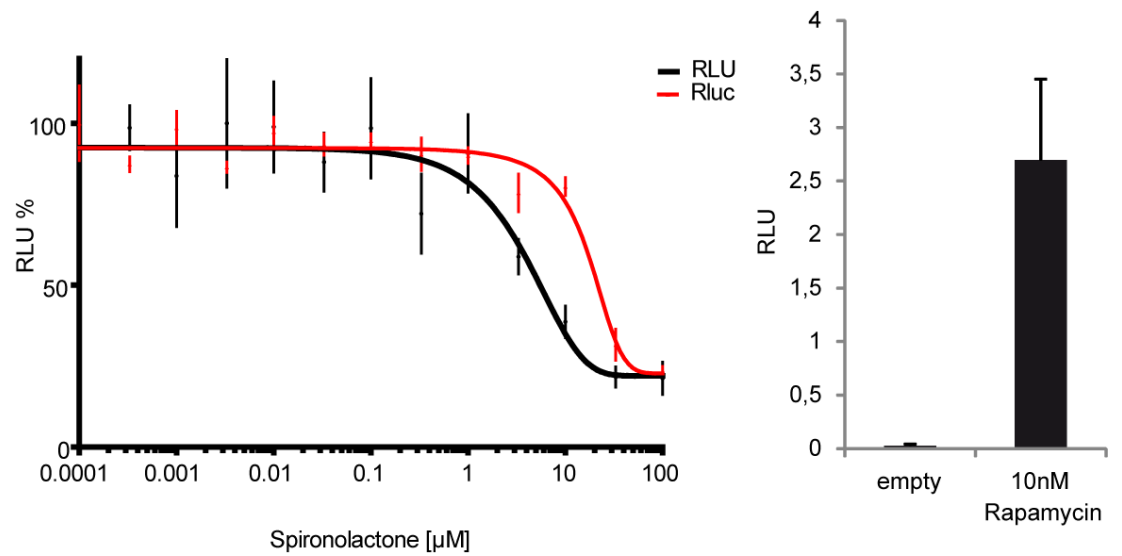

Figure 52: Effect of Spironolactone on the FRB/FKBP interaction induced by Rapamycin

TM-FRB was fused to NTEV-tevS-GV, FKBP was fused to CTEV. PC12 cells were stimulated with 10nM Rapamycin. 
Results
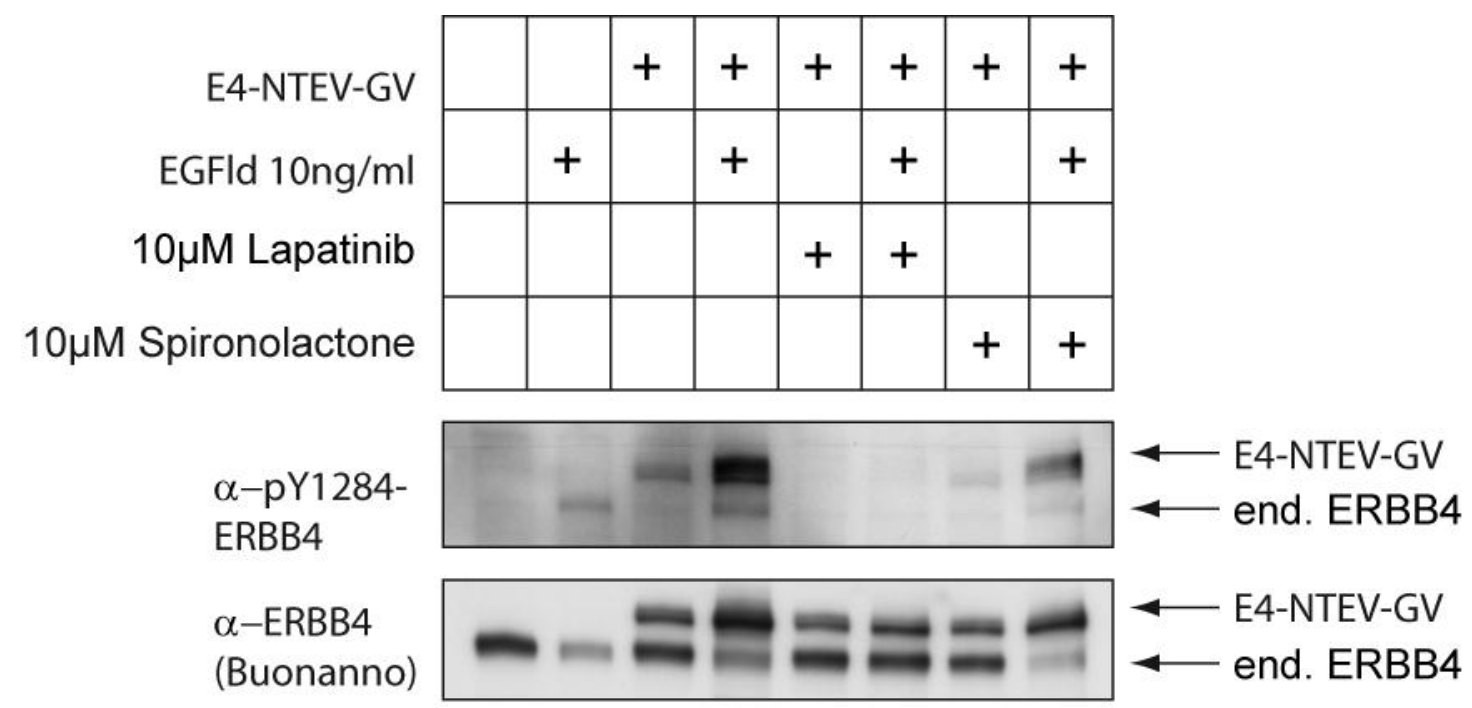

$\alpha-$ Tub

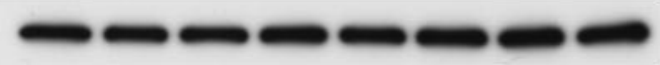

$\alpha-\mathrm{HA}$

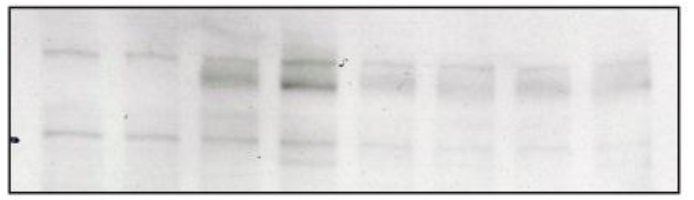

$\alpha-$ ERBB4

$(\mathrm{SC}, \mathrm{rb})$

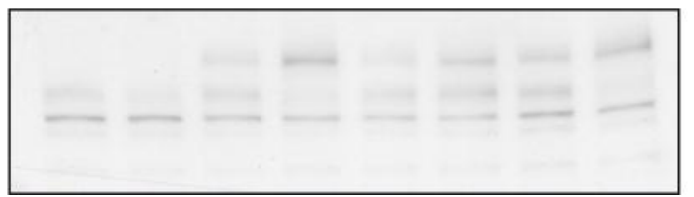

Figure 53: ERBB4 phosphorylation levels are inhibited by Lapatinib and Spironolactone

HEK293 cells were transiently transfected with ERBB4-NTEV-tevS-GV as indicated. Cells were allowed to express the vectors for $24 \mathrm{~h}$, and then stimulated with $10 \mu \mathrm{M}$ Lapatinib, $10 \mu \mathrm{M}$ Spironolactone (Spiro), and 10ng/ml EGF-like domain (EGFld). Cells were then incubated for additional 24h. Protein lysates were blotted and probed against antibodies as indicated. Calculated protein sizes (in kDa): ERBB4, 146.8; ERBB4-Glink-NTEV-tevSGV-2xHA (E4-NTEV-GV), 200. 
Results

\begin{tabular}{|c|c|c|c|c|c|c|c|}
\hline E4-NTEV-GV & & + & + & + & + & + & + \\
\hline EGFld $10 \mathrm{ng} / \mathrm{ml}$ & + & & + & & + & & + \\
\hline $10 \mu \mathrm{M}$ Lapatinib & & & & + & + & & \\
\hline $10 \mu \mathrm{M}$ Spironolactone & & & & & & + & + \\
\hline
\end{tabular}

$\alpha-p Y 1284-E R B B 4$

$\alpha-p Y 1056-E R B B 4$

$\alpha-p Y 984-E R B B 4$

$\alpha-$ ERBB4

(Buonanno)

$\alpha-E R B B 4$ SC

a-pT202 / Y204

-ERK1

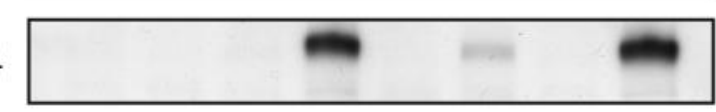

$\alpha$-ERK1

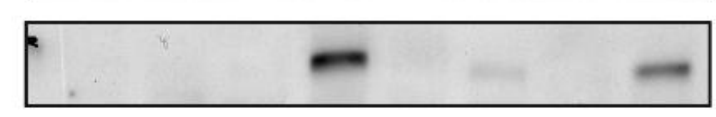

$\alpha-\mathrm{pS6K}$

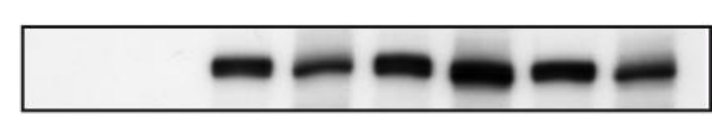

$\alpha-S 6 K$

$\alpha-$ Tub
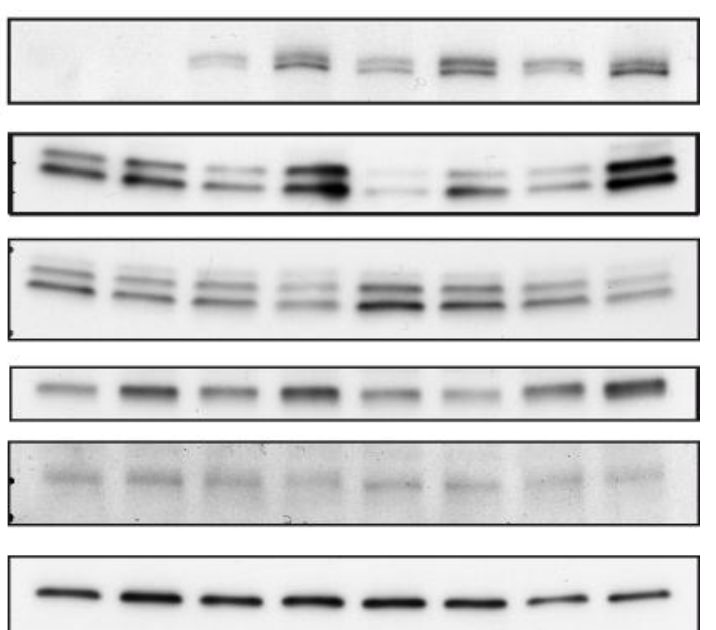

Figure 54: Analysis of phosphorylation levels of ERBB4 and major downstream signalling kinases

ERBB4 phosphorylation is inhibited by Lapatinib and Spironolactone. PC12 cells were transiently transfected with plasmids as indicated. Cells were allowed to express the vectors for $24 \mathrm{~h}$, and then stimulated with $10 \mu \mathrm{M}$ Lapatinib, $10 \mu \mathrm{M}$ Spironolactone (Spiro), and $10 \mathrm{ng} / \mathrm{ml}$ EGF-like domain (EGFld). Cells were then incubated for additional 24h. Protein lysates were blotted and probed against antibodies as indicated. Calculated protein sizes (in kDa): ERBB4-Glink-NTEV-tevS-GV-2xHA (E4-NTEV-GV), 200. (Merge of different blots) 
Results

A

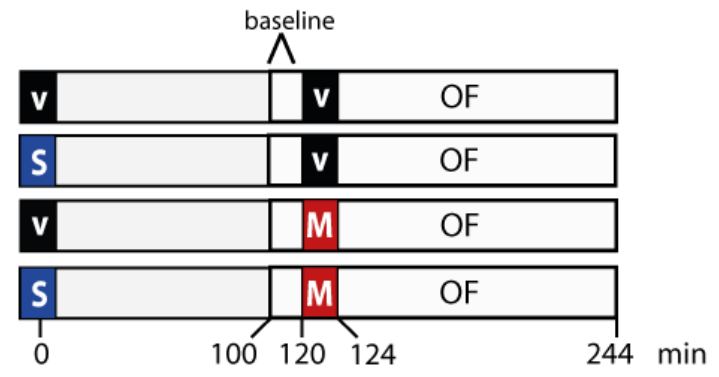

v vehicle

S Spironolactone

M MK801

OF open field

B

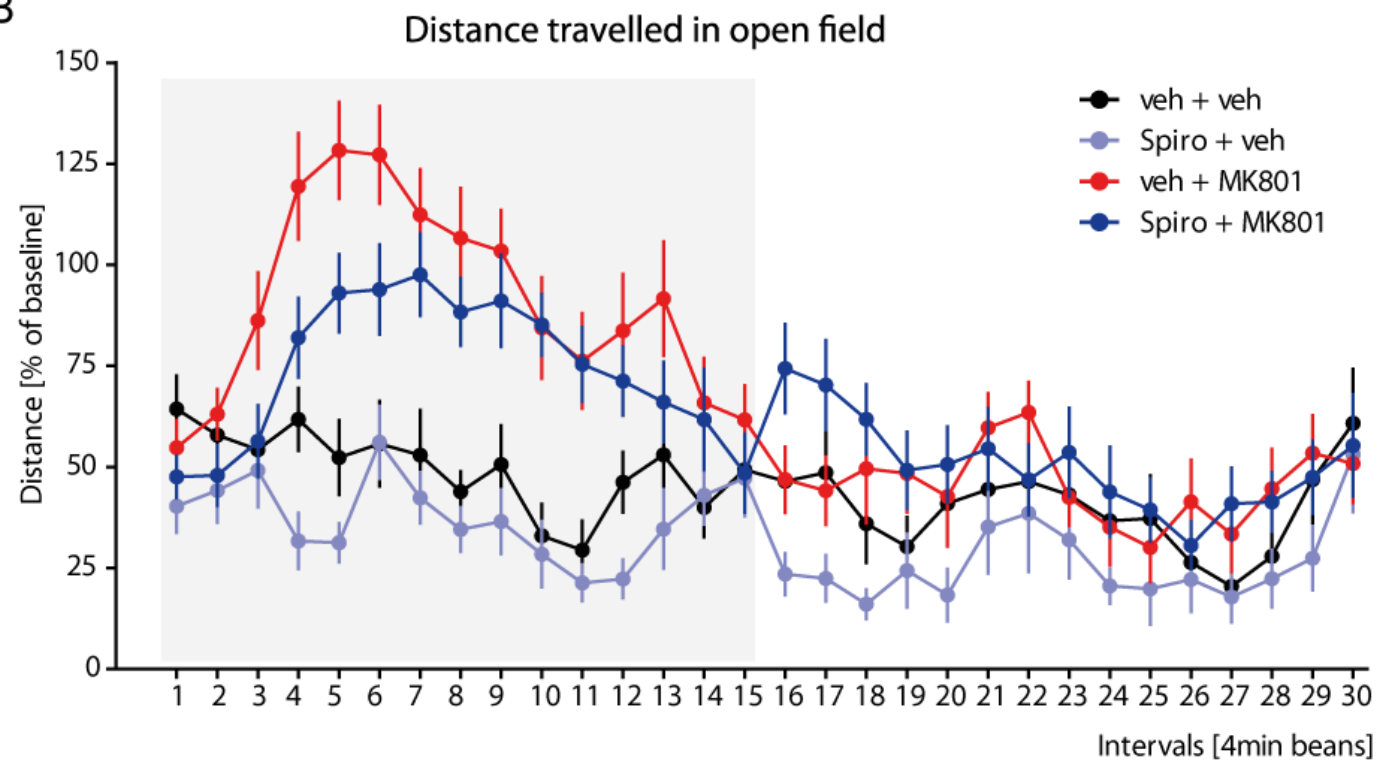

C

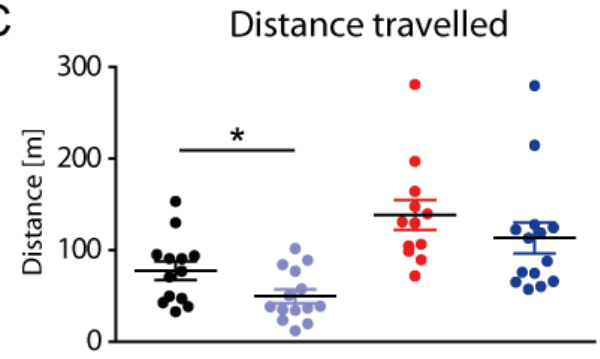

E

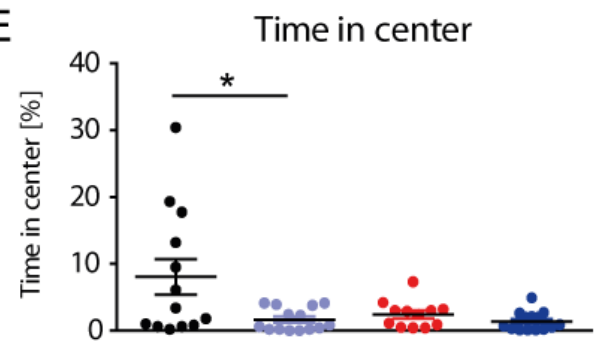

D

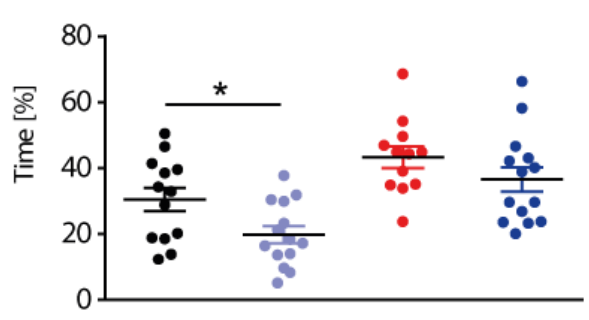

F

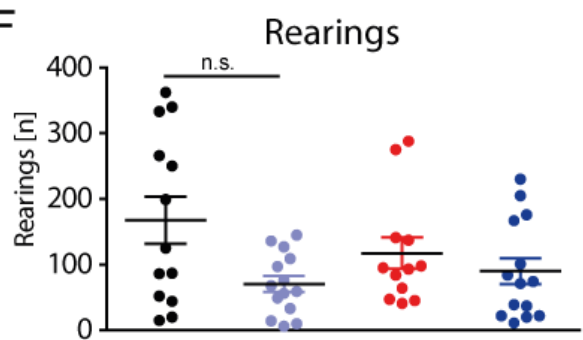

- veh + veh

- Spiro + veh

- veh + MK801

- Spiro + MK801 
Figure 55: Effects of Spironolactone on MK801-induced hyperactivity in mice tested in an open field setup

Effects of Spironolactone on MK801-induced hyperactivity in mice tested in open field.

A) Experimental protocol. 9 weeks old $\mathrm{C} 57 \mathrm{BI} / 6 \mathrm{~N}$ male mice were divided randomly in one of 4 treatment groups: group 1 injected twice with (v) vehicle ([veh+veh], $n=13$ ), group 2 administered with Spironolactone (S) and vehicle ([Spiro+veh], $n=14$ ), group 3 treated with vehicle and (M) MK801 ([veh+MK801], $n=12$ ), and group 4 injected with Spironolactone and MK801 ([Spiro+MK801], $\mathrm{n}=14$ ). $100 \mathrm{~min}$ after the first injection, mice were placed in the open field arena for 20 min to score baseline activity. Thereafter, animals were taken out of the test boxes for $4 \mathrm{~min}$, administered with a second injection, and returned to the open field arena for the next 120 min where activity was monitored.

B) Distance travelled in open field arena depicted as a percentage of mean distance travelled during baseline activity. A 2-way ANOVA was performed pairwise between different treatments during the time window between intervals 1 to 15. [Veh+MK801] animals showed hyperactivity when compared with [veh+veh] controls (interaction time $x$ treatment $F_{(14,322)}=5.73 ; p<0.0001$; effect of treatment $F_{(1,23)}=17.92 ; p=0.0003 ; 2$-way ANOVA). [Spiro+veh] mice showed a non-significant tendency to travelled less distance then [veh+veh] $\left(\mathrm{F}_{(1,25)}=3.39 \mathrm{p}=0.0774,2\right.$-way ANOVA $)$. Animals treated with [Spiro+MK801] displayed a slight tendency to travel shorter distance than mice with induced hyperactivity [veh+MK801] (effect of treatment $F_{(1,24)}=2.89 ; p=0.1018 ; 2$-way ANOVA).

C) Distance travelled in open field. Mice treated with spironolactone [Spiro+veh] travelled less distance than [veh+veh] controls $(49.79 \mathrm{~m} \pm 27.93$ vs $77.76 \mathrm{~m} \pm 36.39 ; p=0.0369)$. [veh+MK801] animals covered longer distance than [veh+veh] $(138.4 \mathrm{~m} \pm 56.65 \mathrm{vs} 77.76$ $m \pm 36.39 ; p=0.0036)$. [Spiro+MK801] animals showed a non-significant tendency to cover a shorter distance than [veh+MK801] mice (113.4 $\mathrm{m} \pm 63.49$ vs $138.4 \mathrm{~m} \pm 56.65 ; p=0.0849$ ). D) Time which mice spent active during open field test. [Spiro+veh] animals spent less time active than control [veh+veh] group $(19.79 \% \pm 9.761$ vs $30.47 \% \pm 12.71 ; p=0.0273)$. [veh+MK801] displayed more activity than [veh+veh] $(43.33 \% \pm 11.47$ vs $30.47 \% \pm 12.71$; $\mathrm{p}=0.0240$ ). [Spiro+MK801] treated group showed non-significant tendency towards less activity when compared with [veh+MK801] mice $(36.57 \% \pm 13.92$ vs $43.33 \% \pm 11.47$; $\mathrm{p}=0.1166$ ).

E) Percentage of time which mice spent in the center of the open field arena. Spironolactone administered with vehicle significantly reduced the percentage of time in which mice were scored in the centre of the open field arena compared to controls ([Spiro+veh] $1.643 \% \pm 1.706$ vs. [veh+veh] $8.046 \% \pm 9.518 ; p=0.0387$ ). Similarly, groups injected with [veh+MK801] and [Spiro+MK801] spent very low percentage of time in the center of test arena $(2.442 \% \pm 2.009$ and $1.371 \% \pm 1.410$, respectively).

F) Number of rearings performed during open field test. Mice treated with spironolactone [Spiro+veh] displayed a non-significant tendency towards less rearings when compared to [veh+veh] controls (70.36 \pm 46.80 vs $167.6 \pm 129.4 ; p=0.0765)$. The [veh+MK801] group displayed similar number of rearings to controls (117.3 \pm 83.32 vs $167.6 \pm 129.4 ; p=0.6053$ ). Spironolactone did not have a significant impact on rearings in the [Spiro+MK801] treated group when compared with the [veh+MK801] group (89.93 \pm 74.79 vs $117.3 \pm 83.32$; $\mathrm{p}=0.2367$ ).

Veh, vehicle; Spiro, Spironolactone; black dots, veh+veh $(n=13)$; light blue dots, Spiro+veh $(n=14)$; red dots, veh+MK801 $(n=12)$; dark blue dots, Spiro+MK801 $(n=14)$. Data presented as mean \pm S.E.M. 


\subsection{Validation Albendazole}

The activator hit found in the screen of the NCC201 library is Albendazole. Albendazole is a Benzimidazole compound normally used as anthelmintic in the treatment of worm infections (van Schalkwyk et al., 1979). It binds to tubulin and inhibits the polymerization of microtubules (Nayak et al., 2011). There is a highly significant activation of luciferase activity in the Nrg1-typel-

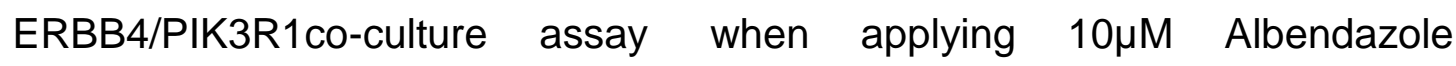
$(p<0,0001)$ (Figure $57 \mathrm{~A})$.

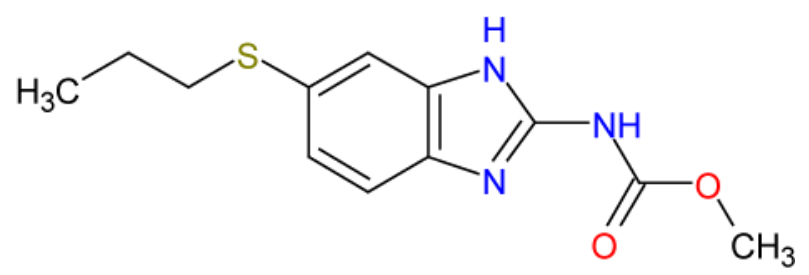

Figure 56: Chemical structure of Albendazole

\subsubsection{Technical controls for Albendazole}

\subsubsection{Renilla Luciferase}

To test for potential Albendazole-mediated toxicity, PC12 cells were transfected with the Renilla luciferase. The test revealed a toxic effect against the co-culture assay at concentrations between $33 \mu \mathrm{M}$ to $100 \mu \mathrm{M}$ (Figure $57 \mathrm{~B}$ ).

\subsubsection{The Gal4-VP16 control assay}

The technical control GV/UAS-Fluc showed a 3-fold increase of $300 \%$ of the Fluc activity. The $\mathrm{EC}_{50}$ of Albendazole is at $0.3 \mu \mathrm{M}$ (Figure $57 \mathrm{G}$ ).

\subsubsection{TEV protease control assay}

To test for any stimulating effects elicited by the TEV protease and its activity at TEV protease cleavage sites, a transmembrane version of the TEV protease (TM-TEV) was co-transfected with a transmembrane-bound GV carrying an internal TEV cleavage site (TM-GV), along with UAS-Fluc and Rluc reporters. Like the GV control assay, the TM-TEV/TM-GV assay showed a comparable 2.5 -foldincrease of the firefly activity, with an $\mathrm{EC}_{50}$ of $1.0 \mu \mathrm{M}$ Albendazole (Figure 57. F)

\subsubsection{Co-culture assay using increasing numbers of Nrg1-typel- expressing cells}

To test Albendazole's activating effect at different pre-activated conditions, the co-culture assay ERBB4/PIK3R1 was stimulated with 1,000, 5,000, and 
10,000 Nrg1-typel-expressing PC12 cells. All three assays roughly showed a 2-fold activation of the firefly luciferase activity, with an $E_{50}$ of $0.3 \mu \mathrm{M}$ Albendazole (Figure $57 \mathrm{C}$ ).

\subsubsection{Single cell assay with soluble Nrg1-derived EGF-like domain}

The single cell ERBB4/PIK3R1 assay was stimulated with 1 or $5 \mathrm{ng} / \mathrm{ml}$ EGFlike domain to get two different pre-activated conditions. Both assays showed a 1.5-fold activation of the firefly luciferase activity, with an $E_{50}$ of $0.6 \mu \mathrm{M}$ Albendazole at a concentration of $1 \mathrm{ng} / \mathrm{ml}$ EGF-like domain, and an $\mathrm{EC}_{50}$ of $0.4 \mu \mathrm{M}$ Albendazole and 5ng/ml EGF-like domain (Figure $57 \mathrm{D}, \mathrm{E}$ ).

The comparison of the data revealed that firefly luciferase activity was highly increased in the technical control assays when Albendazole was applied at high concentrations. For example, the GV technical control assay displayed strongly increased readings for firefly luciferase at Albendazole concentrations that were not toxic for the cells as measured by Renilla luciferase activity (Figure 57 D, D', D"). Therefore, Albendazole seems not to be a specific activator of Nrg1-ERBB4 signalling, but rather an unspecific activator of either $\mathrm{GV}$, the UAS promoter, the activity of firefly luciferase, or a combination thereof. Further validation approaches have to be performed, for example, it should be addressed whether Albendazole has the potential to increase firefly luciferase activity when expressed under the control of the constitutively active CMV promoter (Figure $57 \mathrm{H}$ ).

A

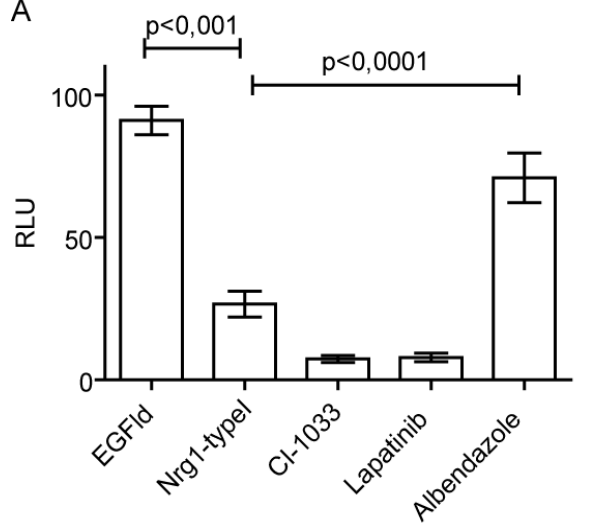

B

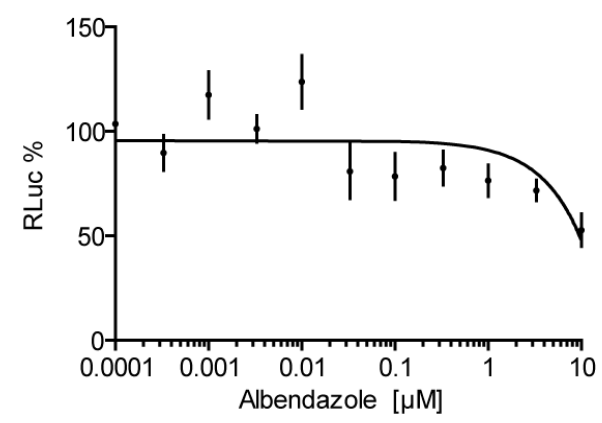

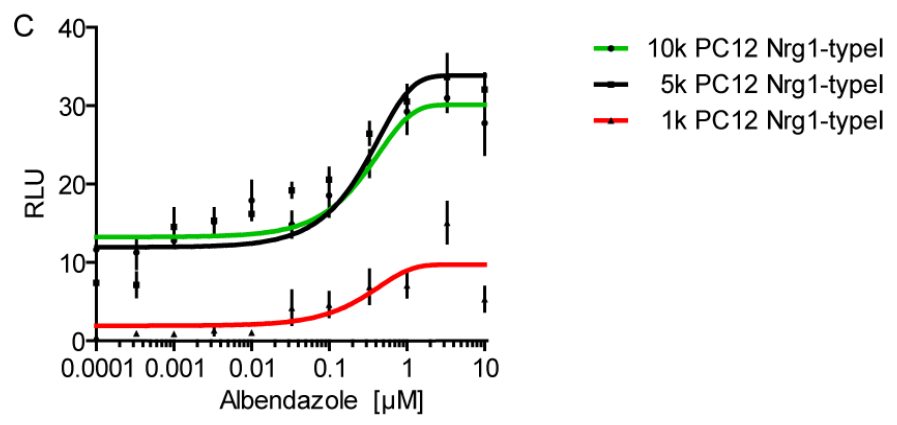



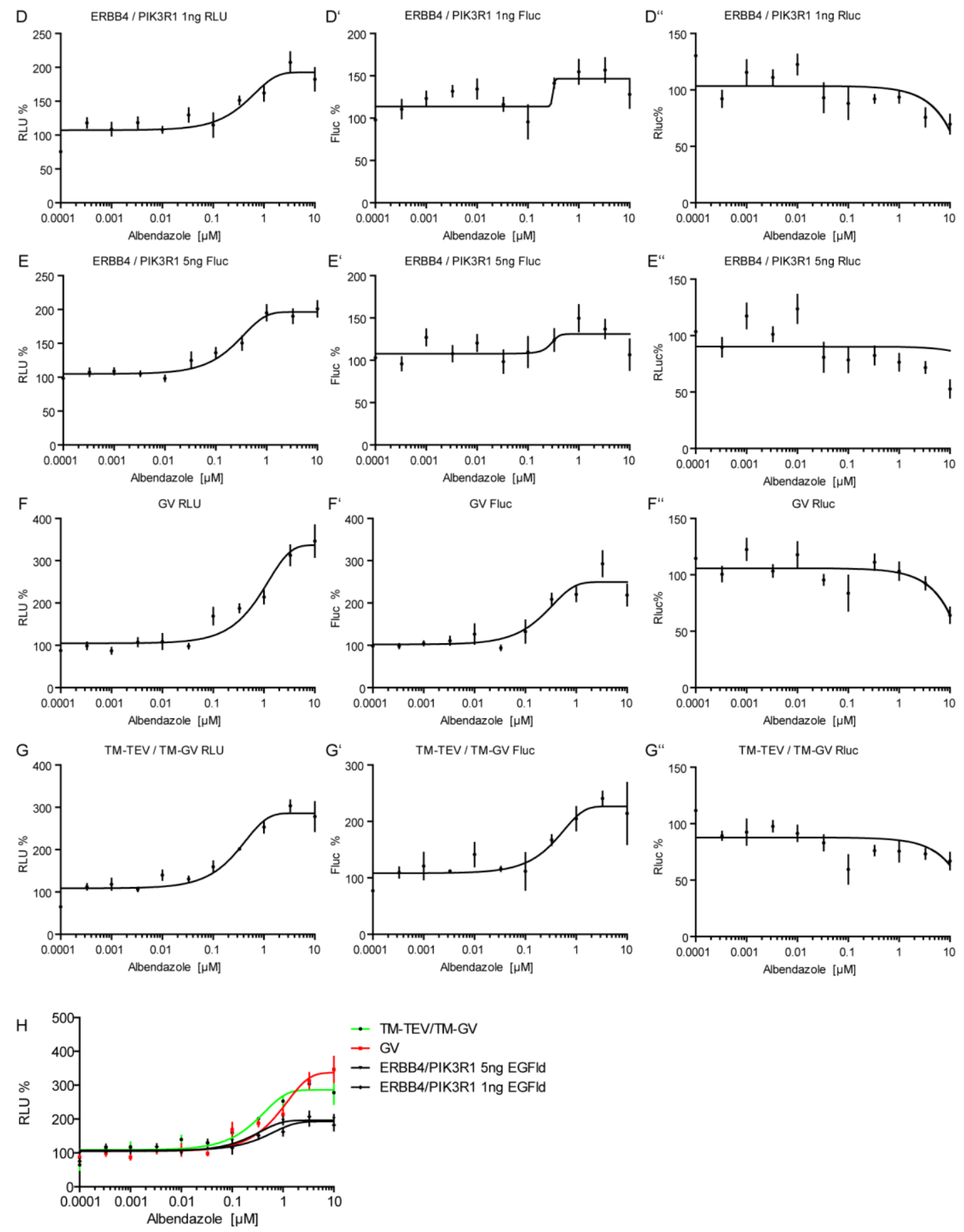

\section{Figure 57: Validation Albendazole}

A) Extracted data from the screen showing a significant activation of Nrg1-ERBB4 signalling caused by Albendazole in the co-culture assay. B) Renilla luciferase assay to test the toxicity of Albendazole. C) ERBB4/PIK3R1 co-culture assays activated with 1k, 5k, or 10k Nrg1-typel cells. D) ERBB4/PIK3R1 single cell assay stimulated with 1ng EGFId. E) ERBB4/PIK3R1 single cell assay stimulated with 5ng EGFld. ERBB4 was fused to NTEVtevS-GV-2HA; Adapters were fused to CTEV-2HA. PC12 cells were stimulated with indicated numbers of Nrg1-typel-expressing cells or indicated cell numbers of EGFId. F) GV/UAS-Fluc technical control assay showing the effect of Albendazole on relative luciferase activity. G) TM-TEV/TM-GV technical control assay showing the effect of Albendazole on TEV protease function. H) Comparison of the data shown from (B) to (I). GV and TM-TEV/TM-GV control assays show the highest activation of relative luciferase activity. 


\subsection{Topotecan}

The NCC003 screen showed a significant reduction in firefly luciferase activity for $10 \mu \mathrm{M}$ Topotecan (brand name Hycamtin, GlaxoSmithKline 2007). Topotecan is a Topoisomerase I inhibitor used as a chemotherapeutic agent for the treatment of ovarian, cervical, and small cell lung cancer.

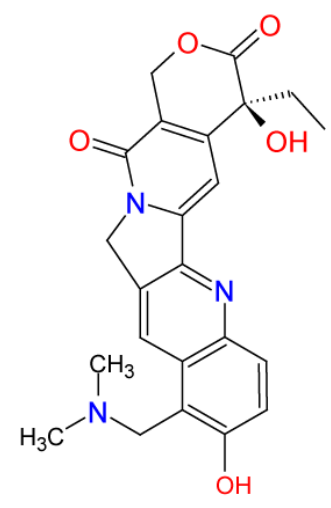

Figure 58: Chemical structure of Topotecan

Topotecan is a drug used as chemotherapeutic. It is a semi-synthetic derivative of the alkaloid Camptothecin, a substance from the bark or leaves of Camptotheca acuminate the Tibetian "Happy Tree". Camptothecin is nearly insoluble in water and has to be modified to increase water solubility. One of these derivatives is Topotecan. It functions as a Topoisomerase I inhibitor, mimicking a DNA base pair, intercalating between the Topoisomerase (Arg364, Asp533, Asn 722) and the DNA (GC base pairs). This leads to a stalling of the rewinding process after the single strand break by stabilising the cleavage complex, and therefore causing double strand breaks that lead to cell cycle arrest in the S-phase, and terminal to apoptosis (Streltsov et al., 2003; Streltsov et al., 2003; Takimoto and Arbuck, 1997a; O'Leary and Muggia, 1998a; Pommier, 2006; Pommier, 2004).

Topotecan was FDA-approved in 2007 and is primarily used in the treatment of ovarian cancer and small cell lung cancer (Carol et al., 2010). It is also tested for brainstem gliomas and sarcomas (Houghton et al., 1995).

In the NCC003 screen, Topotecan was found to decrease significant the luciferase activity ( $p=1,4^{*} 10 \mathrm{e}^{-10}$, two sided, two paired T-Test).

\subsubsection{Vertical validation for Topotecan}

The dose response to increasing concentrations of Topotecan showed an $\mathrm{IC}_{50}$ of $0.5 \mu \mathrm{M}$ in the Nrg1-typel ERBB4/PIK3R1 assay. Exchanging the Nrg1-typel cells with EGFld lead to an $\mathrm{IC}_{50}$ of $0.4 \mu \mathrm{M}$ (Figure 59). 


\subsubsection{Technical controls}

\subsubsection{Renilla luciferase}

The technical validation showed a strong toxic effect of Topotecan between 3.3-100 $\mu \mathrm{M}$ monitoring Renilla luciferase (Figure 60). All values above $10 \mu \mathrm{M}$ are excluded from the dose responses curves, because the decreased Renilla activity leads to false activation in the calculation of the relative luciferase units (RLU).

\subsubsection{Gal4-VP16 control}

In the first technical control assay, UAS-Fluc was co-transfected with GV only. There was no effect of Topotecan below $10 \mu \mathrm{M}$ on the GV/UAS-Fluc control assay. Above 10 $\mathrm{MM}$ Topotecan PC12 cells were dead.

\subsubsection{TEV protease control}

The test showed a mild effect on the TM-TEV/TM-GV system $\left(\mathrm{IC}_{50} 8.5 \mu \mathrm{M}\right)$ but data quality and toxic effects have to be taken into account as well (Figure 60). I can conclude that Topotecan has a strong toxic effect on the cells, but it neither seems to target the firefly luciferase itself, nor to interfere with TEV protease activity.

\subsubsection{Testing different adapter proteins}

The effect of Spironolactone on the recruitment of various other adapter proteins was analysed. The vertical validation of the adapters using the split TEV-based assay showed that the $I_{50}$ for PIK3R1 is at $0.5 \mu \mathrm{M}$, for SRC kinase at $0.9 \mu \mathrm{M}$, for GRB2 at $2.8 \mu \mathrm{M}$, for STAT5A at $2.5 \mu \mathrm{M}$, and for SHC1 at $2.2 \mu \mathrm{M}$. These data suggest that in particular the binding of the adapter molecules PIK3R1 and SRC to the ERBB4 receptor is inhibited by Topotecan, but the difference observed was not as strong as for Spironolactone (Figure 61).

\subsubsection{Horizontal validation}

The horizontal validation showed no inhibitory effect of Topotecan on ERBB1 and ERBB2 homodimers, and on ERBB1/ERBB2 heterodimers stimulated with 10ng/ml EGFld, as they are not activated (Figure 62).

However, Topotecan showed an inhibitory effect on most ERBB homo/heterodimers, with an $\mathrm{IC}_{50}$ of $2.7 \mu \mathrm{M}$ for ERBB1/3, 2.0 $\mu \mathrm{M}$ for ERBB1/4,

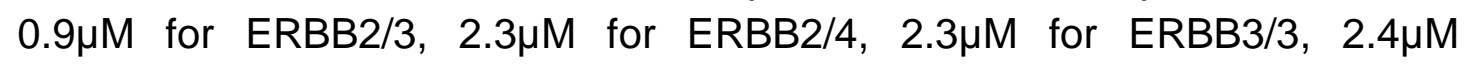
ERBB3/4, and 1.0 $\mu \mathrm{M}$ for ERBB4/4 (Figure 62). ERBB2/3 heterodimers and ERBB4 homodimers are of particular interest, displaying $\mathrm{IC}_{50}$ values of $0.9 \mu \mathrm{M}$ 
and $1.0 \mu \mathrm{M}$, thus having $\mathrm{IC}_{50}$ values that are within the range of the inhibitory effect for ERBB4/PIK3R1 and ERBB4/SRC assays (Figure 63).

\subsubsection{ERBB2/ERBB4 dimerisation and adapters}

To get further insight into Spironolactone's specificity towards the potential inhibition of ERBB2/4, the adapter recruitment for ERBB2/4 heterodimers was analysed. The validation of ERBB2/4 adapter recruitment showed $\mathrm{IC}_{50}$ values of $0.6 \mu \mathrm{M}$ for ERBB2/4/SHC, $0.7 \mu \mathrm{M}$ for ERBB2/4/GRB2, $0.8 \mu \mathrm{M}$ for ERBB2/4/SRC, $2.1 \mu \mathrm{M}$ for ERBB2/4/PIK3R1, and $0.7 \mu \mathrm{M}$ for ERBB2/4/STAT5A (Figure 65).

\subsubsection{ERBB2/ERBB3 dimerisation and adapters}

Likewise, the adapter recruitment for ERBB2/3 heterodimers was measured. The validation of ERBB2/3 adapter recruitment showed $\mathrm{IC}_{50}$ values of $0.8 \mu \mathrm{M}$ for ERBB2/3/SHC1, 2.3 $\mu \mathrm{M}$ for ERBB2/3/GRB2, 1.7 $\mu \mathrm{M}$ ERBB2/3/SRC, $1.1 \mu \mathrm{M}$ for ERBB2/3/PIK3R1, and $0.8 \mu \mathrm{M}$ for ERBB2/3/STAT5A (Figure 64).

Topotecan inhibits all measured ERBB homo and heterodimerisations within a comparable range. Therefore, Topotecan is not an ERBB4-specific inhibitor.

\subsubsection{Orthogonal validation}

\subsubsection{ERBB1/ERBB1/EGF}

ERBB1/1 (EGFR-homodimerisation) stimulated with 1ng/ml EGF showed an inhibition of the homodimerisation at $\mathrm{IC}_{50}$ values of $0.5 \mu \mathrm{M}$, but the inhibition seemed to be incomplete (Figure 67).

\subsubsection{FRB/FKBP model interaction induced by Rapamycin}

To address whether Topotecan is specific to the inhibition of ERBB receptormediated signalling, I tested Topotecan's effect in a another split TEV assay that used the Rapamycin-regulated model interaction of a membrane-targeted FRB fused to the NTEV moiety (TM-FRB-NTEV) and a cytosolic FKBP fused to the CTEV moiety (FKBP-CTEV).

TM-FRB-NTEV and FKBP-CTEV were pre-stimulated with 10nM Rapamycin to ensure occurred interaction. Addition of Topotecan showed no effect, suggesting that it does not affect protein-protein interactions in general (Figure 68).

\subsubsection{ERBB4/PIK3R1 assay and effects caused by Irinotecan and SN38}

Irionotecan, a second-generation drug of Camptothecin (i.e. Topotecan derivative), was also present in the NCC003 library. Of interest, Irinotecan had 
no effect on the ERBB4/PIK3R1 co-culture assay. Irinotecan is a pro-drug that has to be activated in the liver (Xie et al., 2003). As the co-culture assay cannot provide a metabolic functionality that is present in the liver, I tested the activated Irinotecan-metabolite SN38 (Chabot 1998). SN38 did not show any inhibitory or activatory activity in the ERBB4/PIK3R1 co-culture assay (Figure 66). The analysis of Irinotecan and SN 38 suggests that the measured effect is Topotecan-specific and not a general effect of Camptothecin analogues.

\subsubsection{Summary validation Topotecan}

\begin{tabular}{|c|c|c|c|}
\hline \multicolumn{3}{|c|}{ Technical validation } & $\mathrm{IC}_{50}$ \\
\hline & Rluc & & n.c. \\
\hline & GV & & n.c. \\
\hline TM-TEV & TM-GV & & 8.5 \\
\hline NTEV-tevS-GV & CTEV & & $I_{50}$ \\
\hline ERBB4 & ERBB4 & & 1.0 \\
\hline \multirow[t]{2}{*}{ ERBB3 } & ERBB4 & & 2.4 \\
\hline & ERBB3 & & 2.3 \\
\hline \multirow[t]{3}{*}{ ERBB2 } & ERBB4 & & 2.3 \\
\hline & ERBB3 & & 0.9 \\
\hline & ERBB2 & & n.c. \\
\hline \multirow[t]{4}{*}{ ERBB1 } & ERBB4 & & 2.0 \\
\hline & ERBB3 & & 2.7 \\
\hline & ERBB2 & & n.c. \\
\hline & ERBB1 & & n.c. \\
\hline NTEV-tevS-GV & CTEV & & $I C_{50}$ \\
\hline \multirow[t]{5}{*}{$\begin{array}{l}\text { ERBB4 } \\
\text { homodimerisation }\end{array}$} & SHC1 & & 2.2 \\
\hline & GRB2 & & 2.8 \\
\hline & SRC & & 0.9 \\
\hline & PIK3R1 & & 0.4 \\
\hline & STAT5A & & 2.5 \\
\hline V5tag & NTEV-tevs-GV & CTEV & $I C_{50}$ \\
\hline \multirow[t]{5}{*}{ ERBB2 } & ERBB3 & SHC1 & 0.8 \\
\hline & ERBB3 & GRB2 & 2.3 \\
\hline & ERBB3 & SRC & 1.7 \\
\hline & ERBB3 & PIK3R1 & 1.1 \\
\hline & ERBB3 & STAT5A & 0.8 \\
\hline \multirow[t]{5}{*}{ ERBB2 } & ERBB4 & SHC1 & 0.6 \\
\hline & ERBB4 & GRB2 & 0.7 \\
\hline & ERBB4 & SRC & 0.8 \\
\hline & ERBB4 & PIK3R1 & 2.1 \\
\hline & ERBB4 & STAT5A & 0.7 \\
\hline
\end{tabular}




\begin{tabular}{|l|l|l|r|} 
NTEV-tevS-GV & CTEV & Drug & IC $_{50}$ \\
\hline ERBB1 & ERBB1 & EGF & 0.5 \\
\hline ERBB4 & PIK3R1 & co-culture assay & \\
\hline ERBB4 & PIK3R1 & SN38 & 0.4 \\
\hline
\end{tabular}

Table 16: Summary of $\mathrm{IC}_{50}$ values

From these data, Topotecan was found to be an inhibitor of ERBB dimerisation and adapter recruitment. Binding of Topotecan to ERBB receptors has to be further validated. However, the severe side effects of Topotecan, resulting from its anti-topoisomerase I effects, makes the usage of Topotecan for the treatment of psychiatric patients highly unlikely (Alimonti et al., 2004). To use the potential of Topotecan in this setting, an in-depth analysis of the underlying mechanisms related to the toxicity has to be performed. Only then, it might be worth to look at new lead structures for further developments.

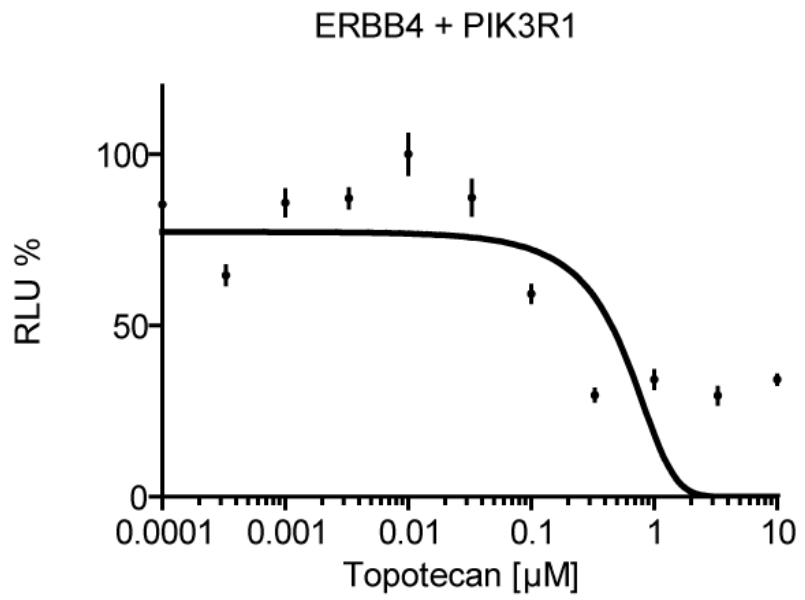

Figure 59: ERBB4/PIK3R1 co-culture assay

A) ERBB4 was fused to NTEV-tevS-GV-2HA; PIK3R1 was fused to CTEV-2HA. Cells were stimulated with $10 \mathrm{ng} / \mathrm{ml}$ EGFld. 
Results

A

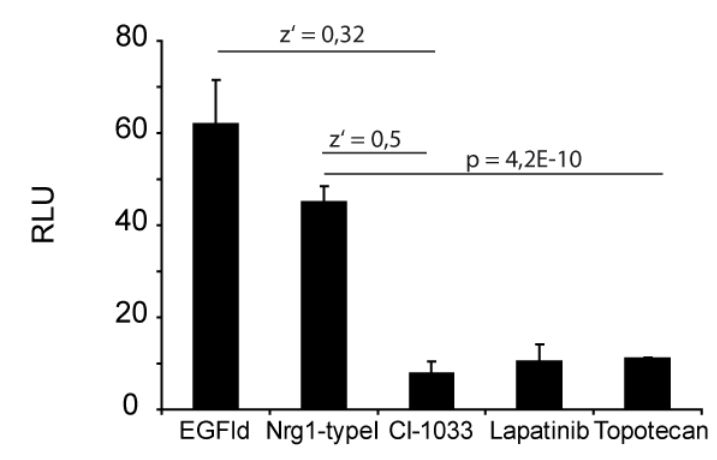

C

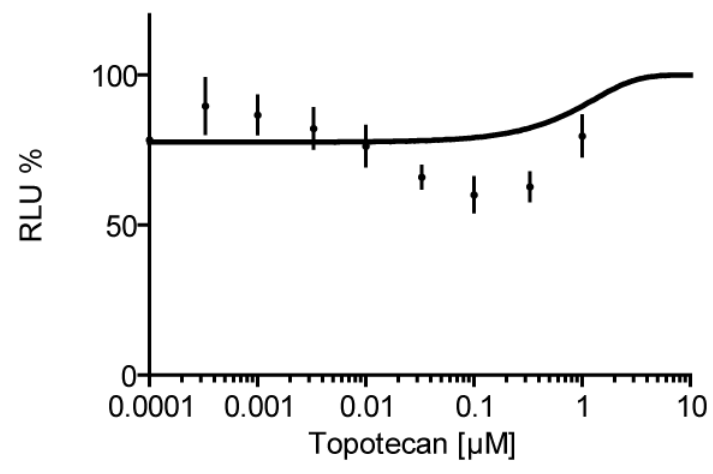

B

RLU

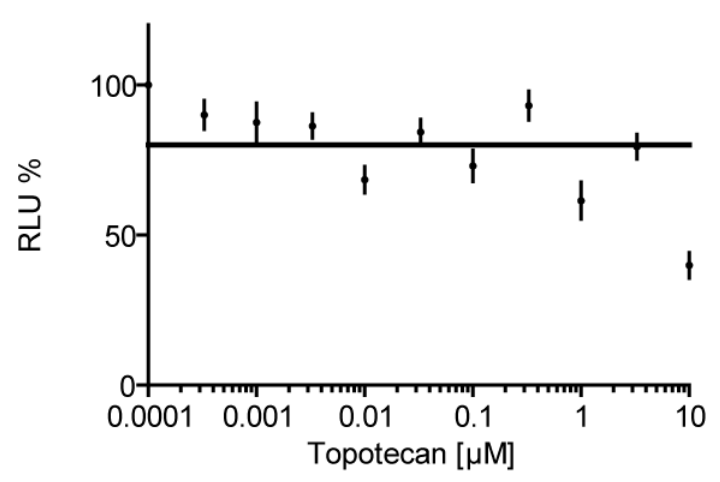

D

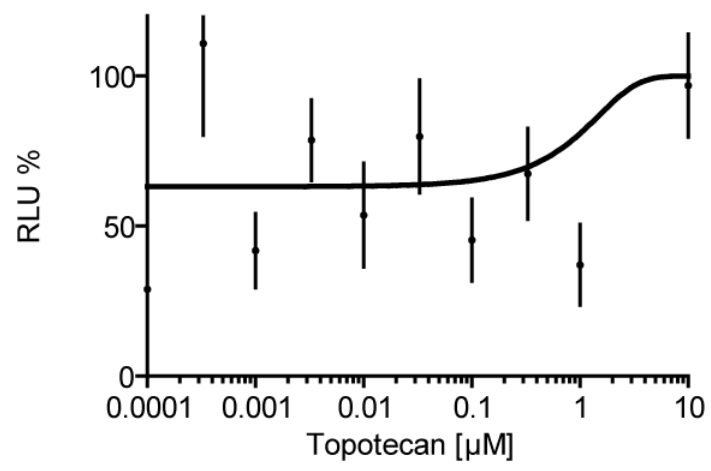

E

Merge technical controls

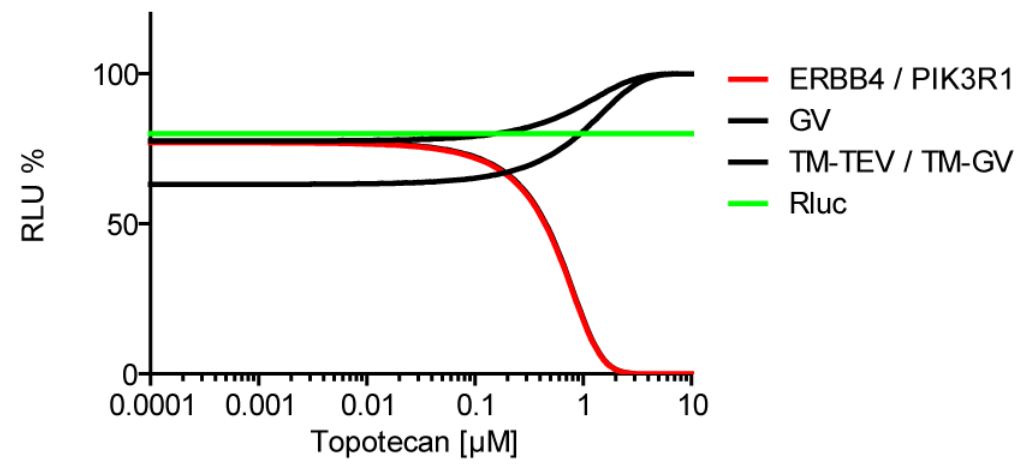

Figure 60: Technical controls for Topotecan

A) Data set from the NCC003 screen. Topotecan significantly inhibits Nrg1-typelstimulated ERBB4/PIK3R1signalling. B) Renilla luciferase assay. Cells were transfected with 20ng/well TK-Rluc. C) GV control assay in PC12 cells. Cells were transfected with 20ng/well CMV-GV, UAS-Fluc, and TK-Rluc. D) TM-TEV/TM-GV control assay in PC12 cells. Cells were transfected with 20ng/well TM-TEV and TM-GV.E) Datasets shown from $(B)$ to $(D)$ were merged. The ERBB4/PIK3R1 assay is shown in red. 
A

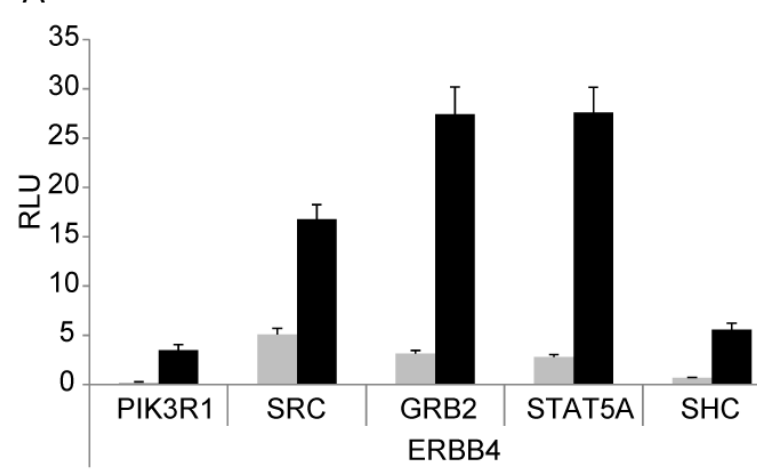

C

ERBB4 / SHC

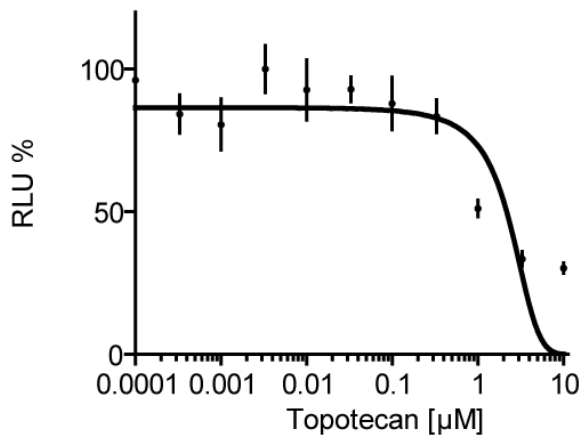

$\mathrm{E}$

ERBB4 / GRB2

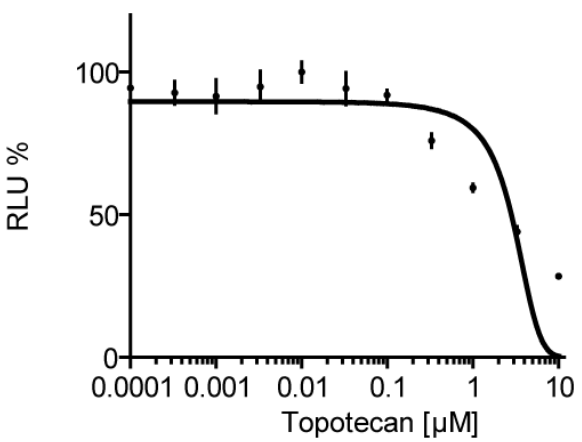

G

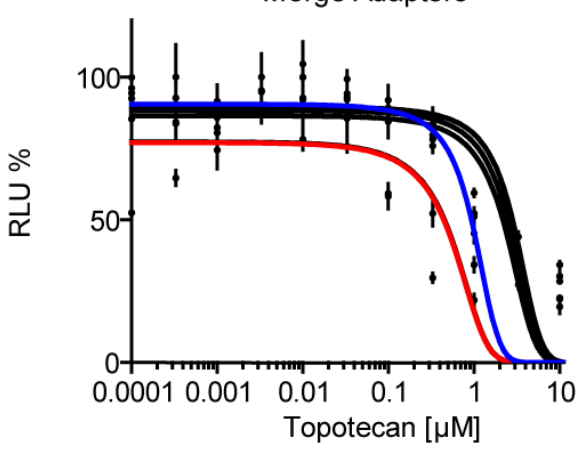

B

ERBB4 / PIK3R1

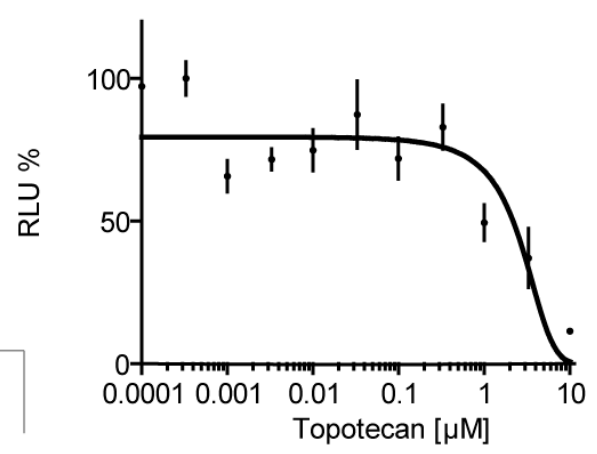

D

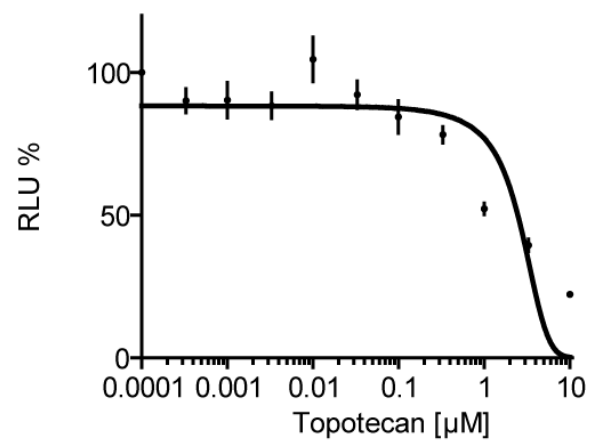

$\mathrm{F}$

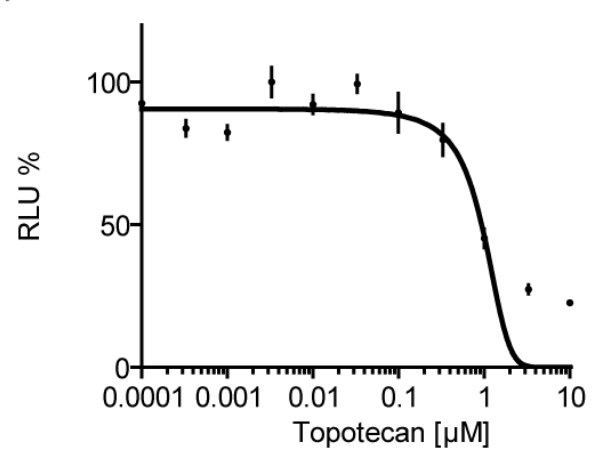

Figure 61: Vertical validation Topotecan ERBB4/adapter recruitment

A) ERBB4/adapter recruitment. ERBB4 was fused to NTEV-tevS-GV-2HA; adapters were fused to CTEV-2HA. Cells were stimulated with $10 \mathrm{ng} / \mathrm{ml}$ EGFld. B-F) ERBB4/adapter recruitment. ERBB4 was fused to NTEV-tevS-GV-2HA; adapters were fused to CTEV2HA. PC12 cells were stimulated with $10 \mathrm{ng} / \mathrm{ml}$ EGFld. G) Comparison of ERBB4/adapter recruitment assays to ERBB4-PIK3R1 recruitment assay (red). ERBB/adapter recruitment for SHC1, GRB2, and STAT5A are less efficiently inhibited compared to the ERBB4/PIK3R1 recruitment. 
Results

A

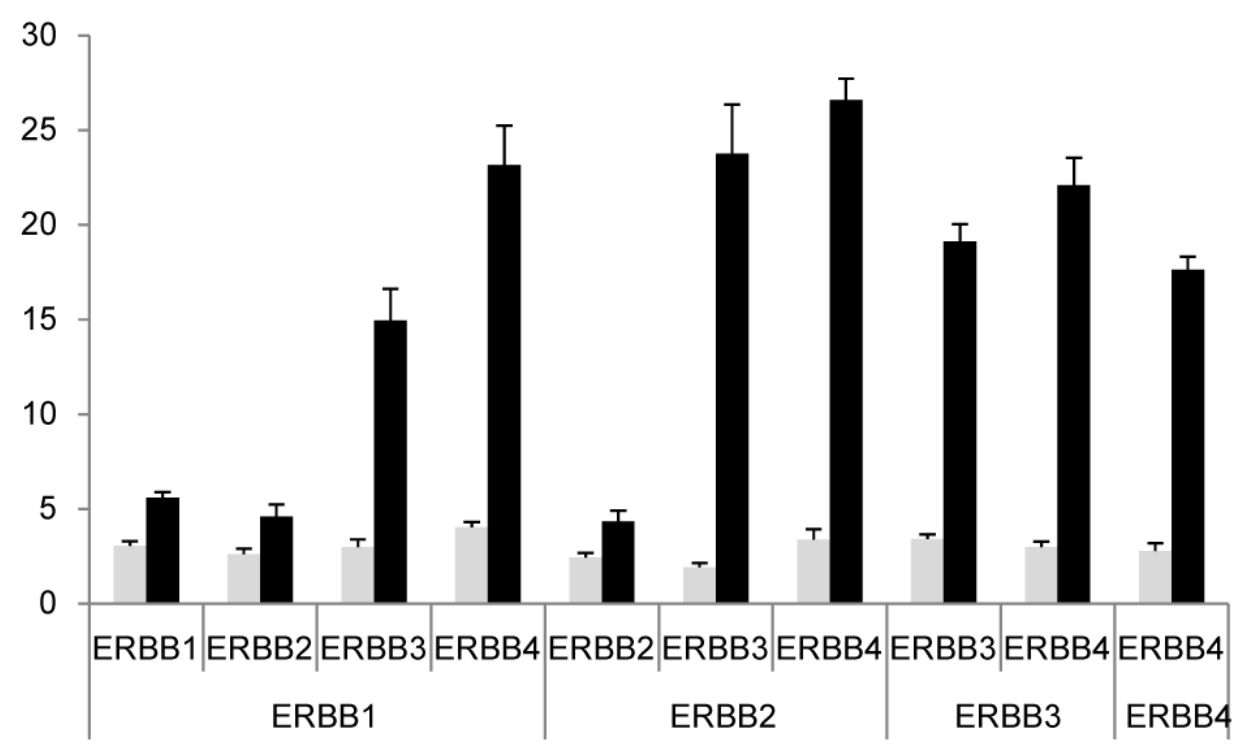

B

ERBB1 / ERBB1

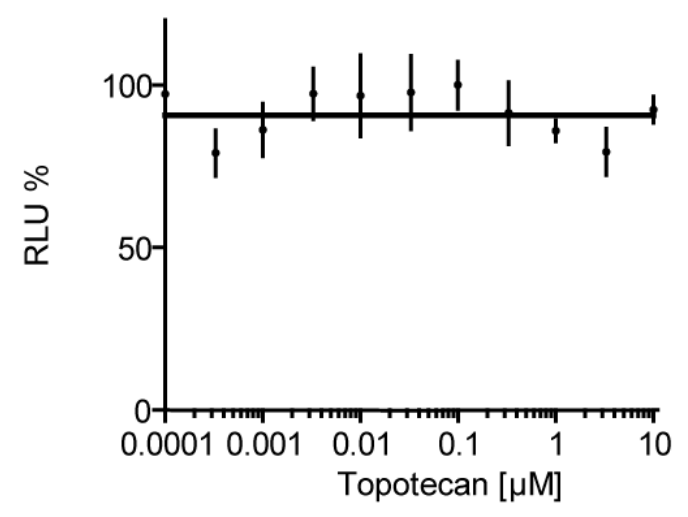

C

ERBB1 / ERBB2

D

ERBB1 / ERBB3

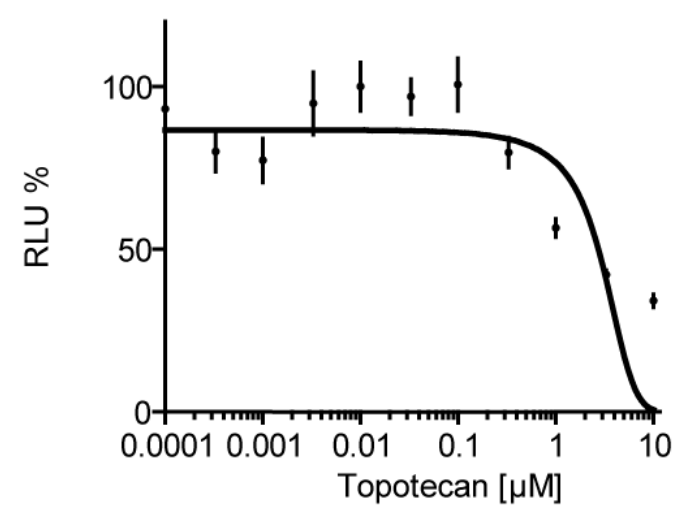

E

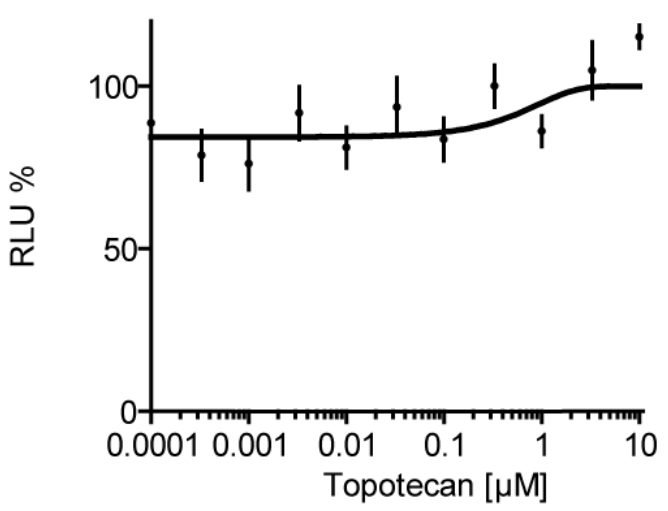

ERBB1 / ERBB4

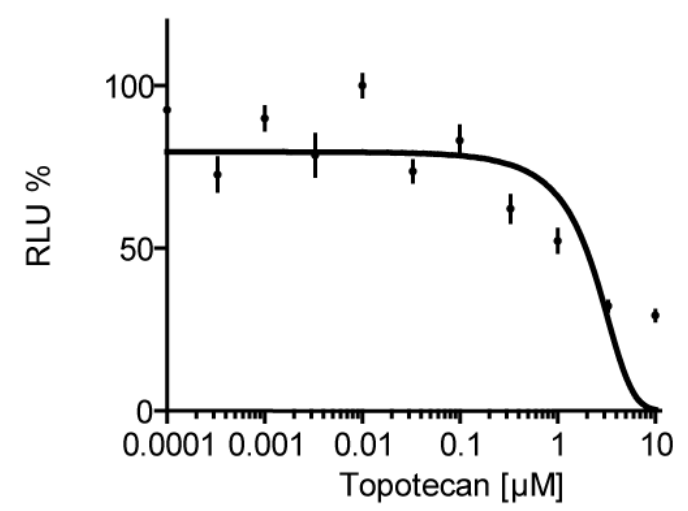


$\mathrm{F}$

ERBB2 / ERBB2

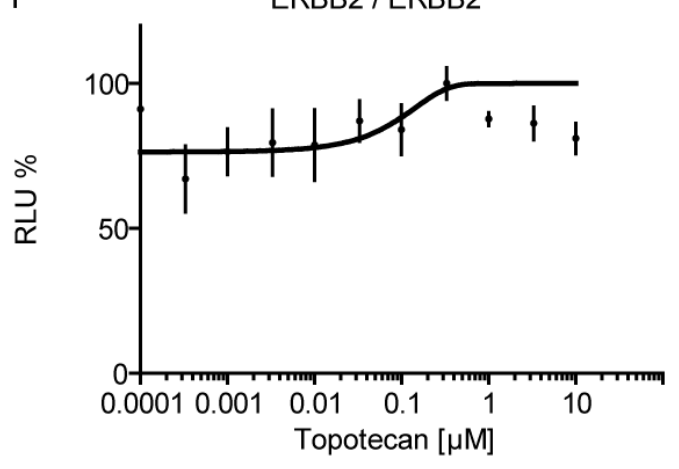

$\mathrm{H}$
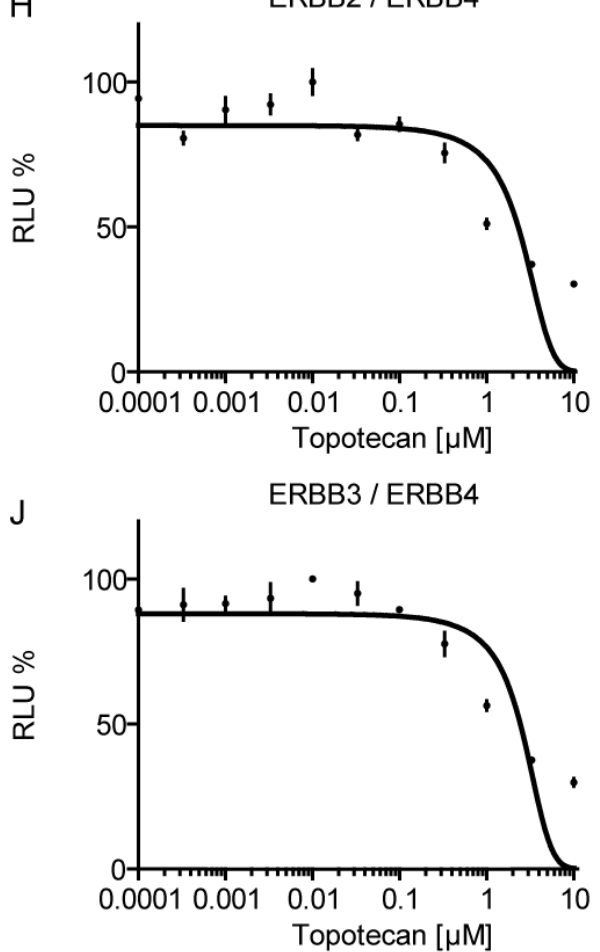

G

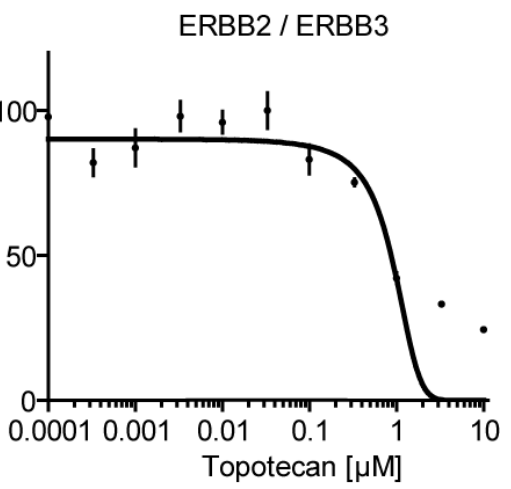

I

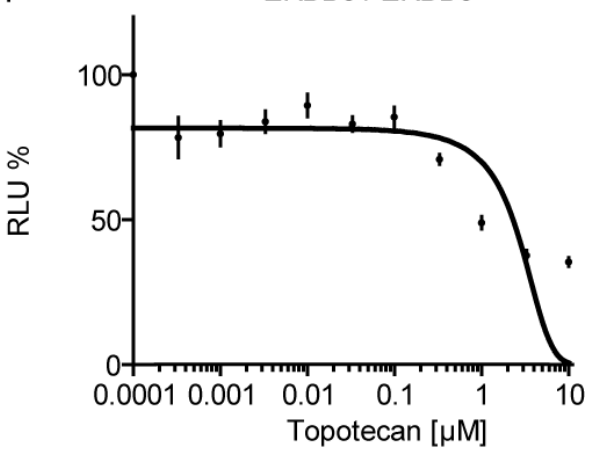

K

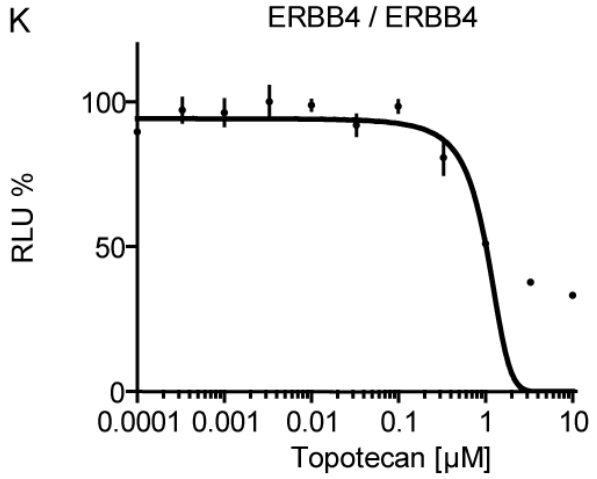

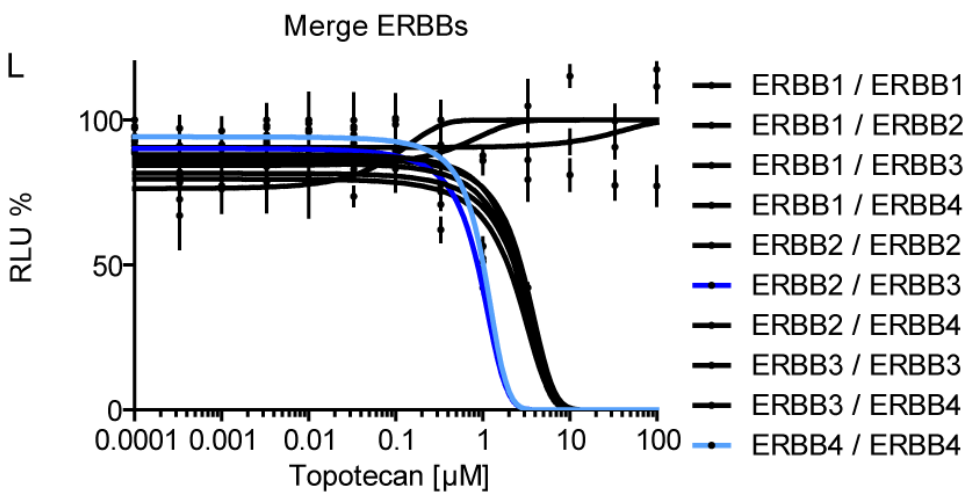

Figure 62: Homo and heterodimerisation of ERBB receptors

A) ERBB homo- and heterodimerisation. ERBBx/ERBBy split TEV assay in PC12 cells. ERBBx was fused to NTEV-tevS-GV-2HA; ERBBy was fused to CTEV-2HA. Cells were stimulated with $10 \mathrm{ng} / \mathrm{ml}$ EGFld. B-K) ERBB homo- and heterodimerisations. ERBBx was fused to NTEV-tevS-GV-2HA; ERBBy was fused to CTEV-2HA. PC12 cells were stimulated with $10 \mathrm{ng} / \mathrm{ml}$ EGFld. L) Comparison of ERBB homo- and heterodimerisation assays to ERBB4-PIK3R1 recruitment. ERBB homo/heterodimerisations are less efficiently inhibited compared to ERBB4-PIK3R1 recruitment. 
Results

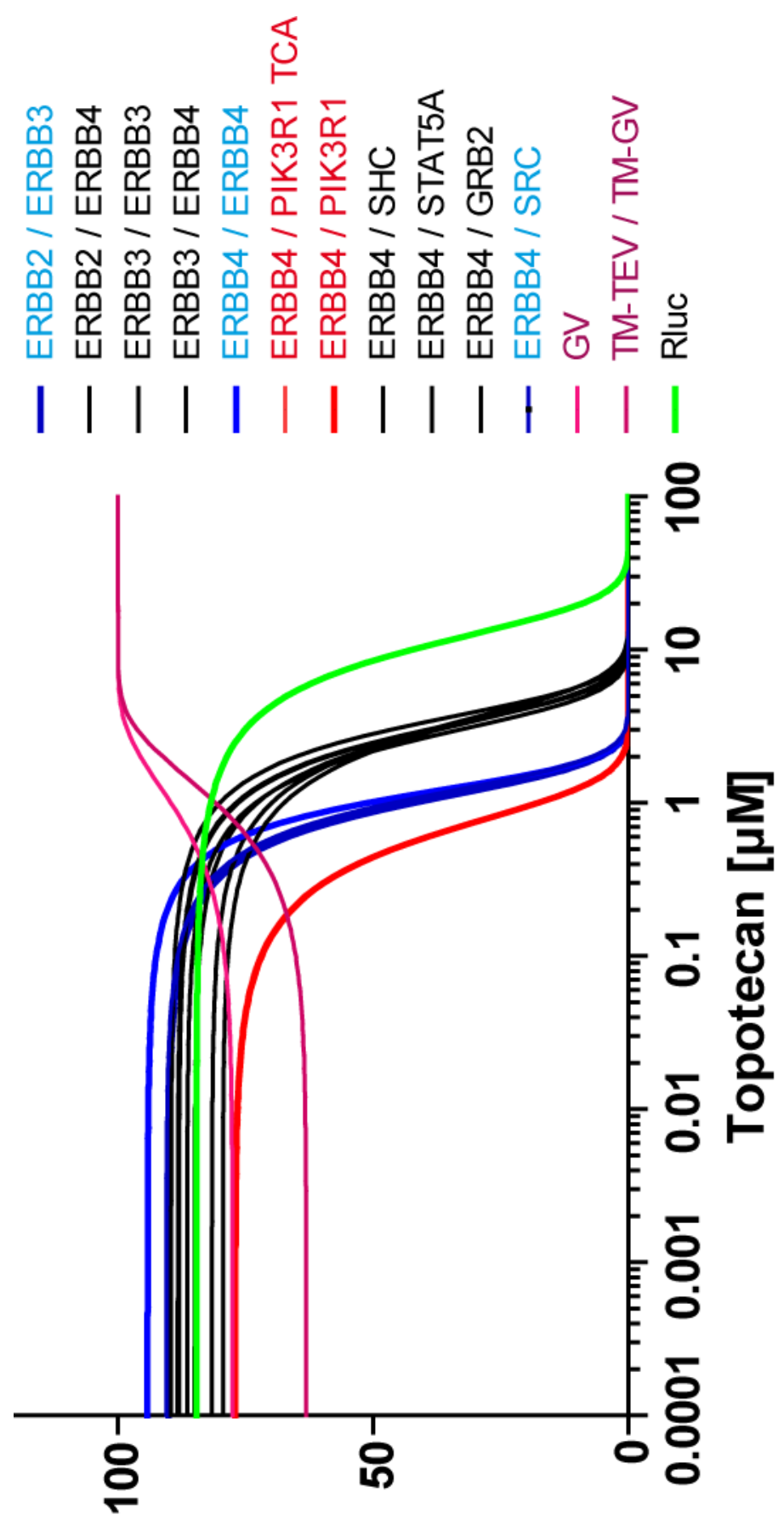

\section{\%}

Figure 63: Summary on Topotecan validation 
A

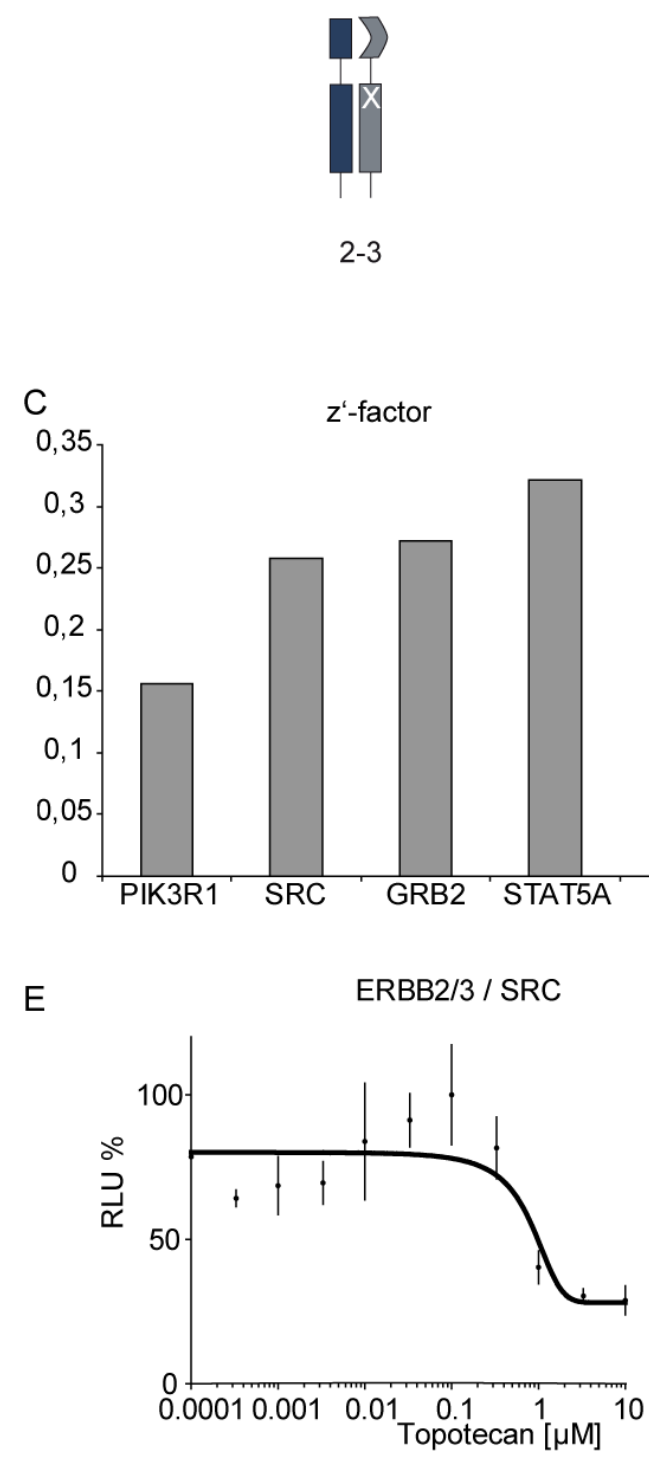

G

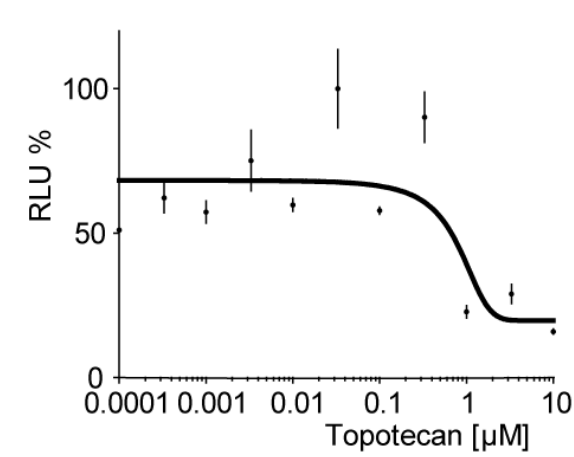

B 140

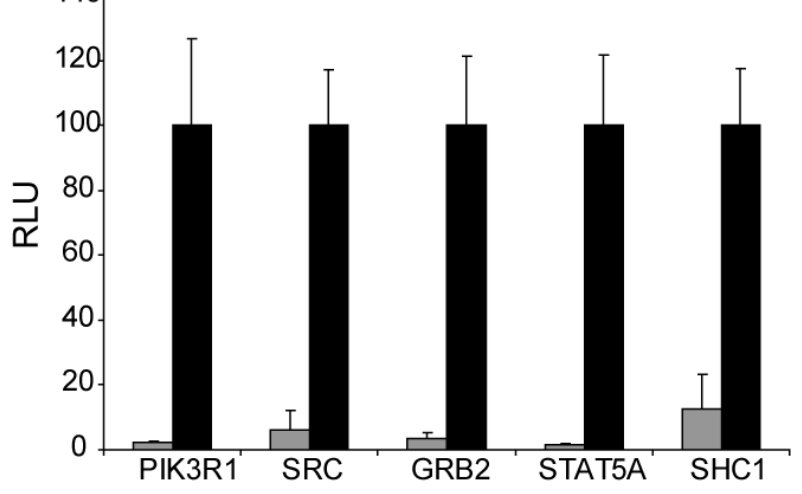

D ERBB2/3 / PIK3R1

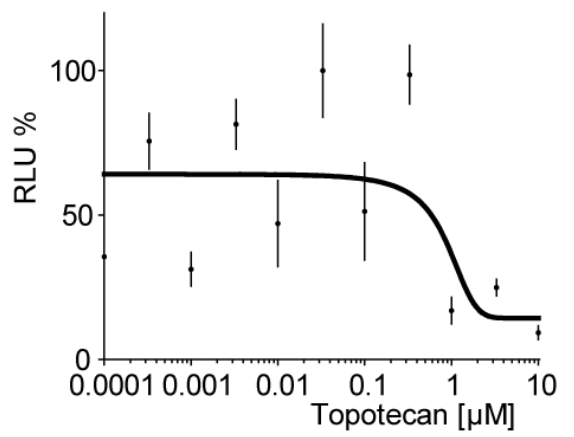

F

ERBB2/3 / GRB2

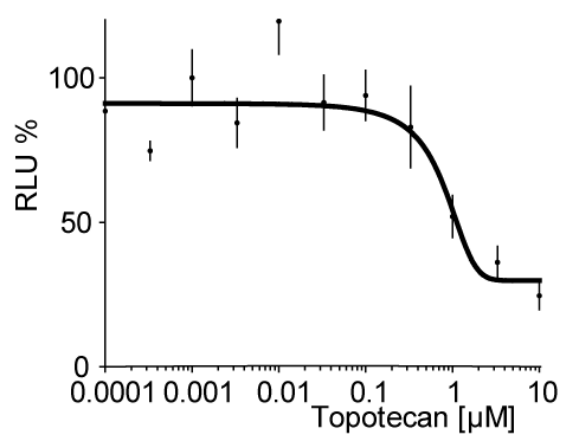

$\mathrm{H}$

ERBB2/3 / SHC1

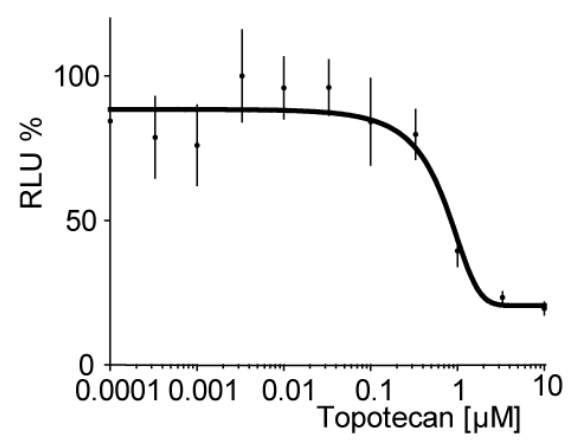

Figure 64: Horizontal validation ERBB2/ERBB3/adapters

A) Schematic representation ERBB2/ERBB3 heterodimerisation. B) Assay performance. ERBB2/ERBB3 heterodimerise after stimulation with $10 \mathrm{ng} / \mathrm{ml}$ EGFld and recruit adapters as indicated. C) Comparison of the $z$ '-factors obtained from the assays. D-H) ERBB2/ERBB3/various adapters. ERBB2 was fused to a V5 tag, ERBB3 was fused to NTEV-tevS-GV-2HA; adapters were fused to CTEV-2HA. PC12 cells were stimulated with $10 \mathrm{ng} / \mathrm{ml}$ EGFld. 
A

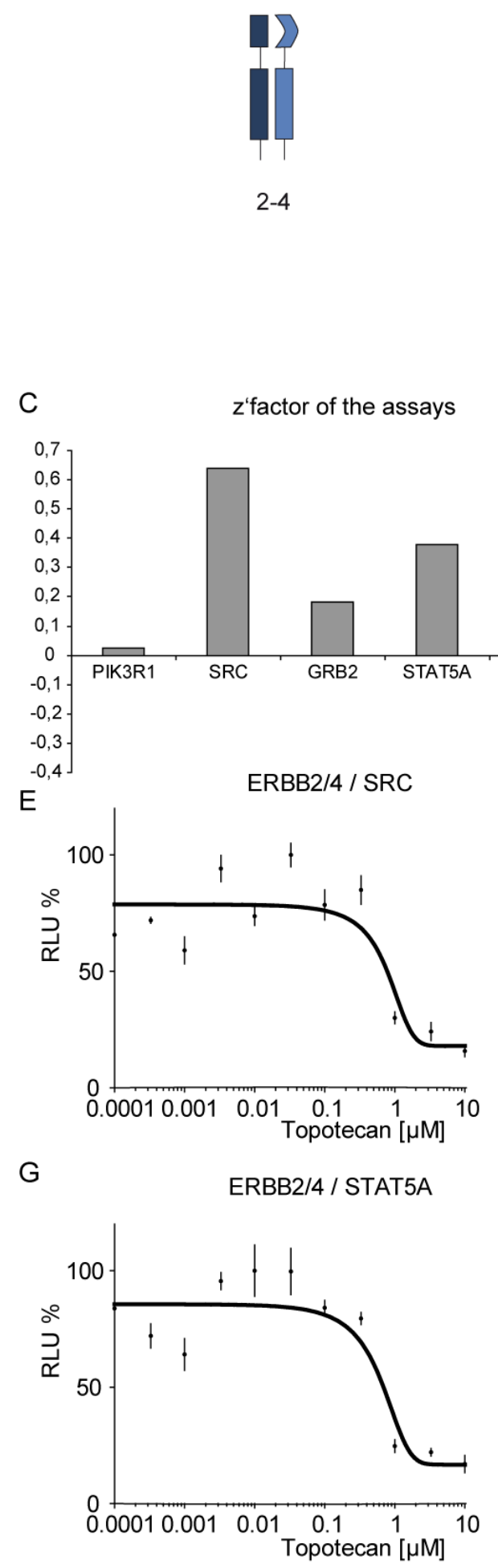

B

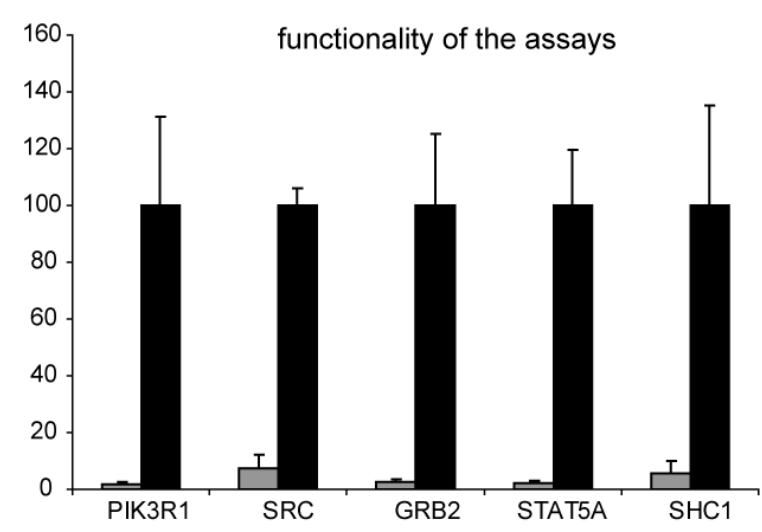

D

ERBB2/4 / PIK3R1

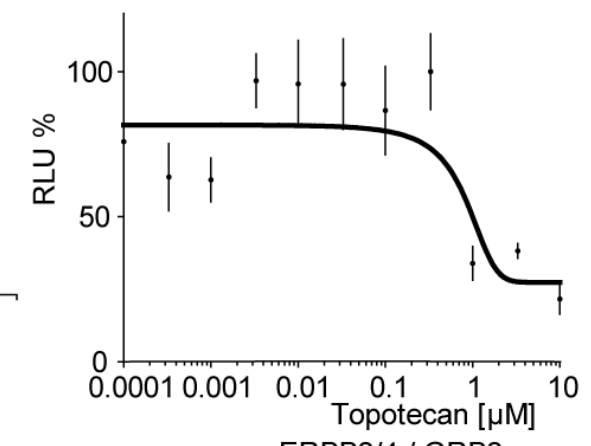

$\mathrm{F}$ ERBB2/4 / GRB2

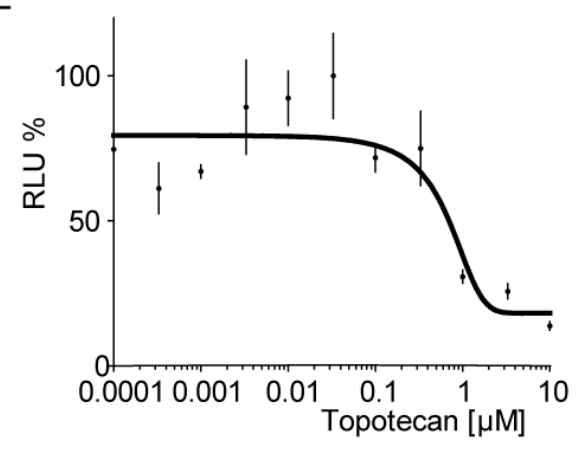

$\mathrm{H}$

ERBB2/4 / SHC1

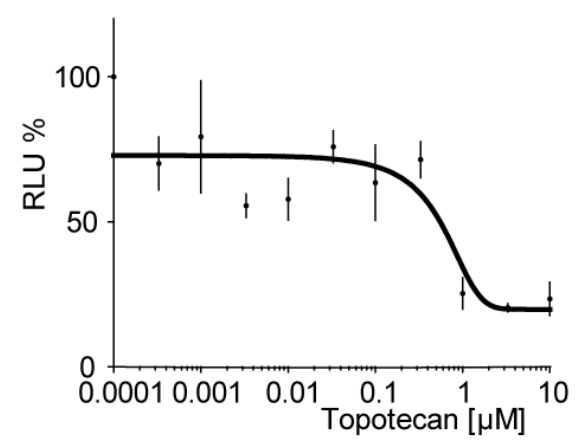

Figure 65: Horizontal validation for Topotecan's effect on ERBB2/ERBB4/adapters

A) Schematic representation ERBB2/ERBB4 heterodimerisation. B) Assay performance. ERBB2/ERBB4 heterodimerise after stimulation with $10 \mathrm{ng} / \mathrm{ml}$ EGFld and recruit adapters as indicated. C) Comparison of the $z$ '-factors obtained from the assays. D-H) ERBB2/ERBB4/various adapters. ERBB2 was fused to a V5 tag, ERBB4 was fused to NTEV-tevS-GV-2HA; adapters were fused to CTEV-2HA. PC12 cells were stimulated with $10 \mathrm{ng} / \mathrm{ml}$ EGFld. 
A Camptothecin

Topoisomerase I inhibitor

Camptotheca acuminata<smiles>CC[C@@]1(O)C(=O)OCc2c1cc1n(c2=O)Cc2cc3ccccc3nc2-1</smiles>

Topotecan

Topoisomerase I inhibitor

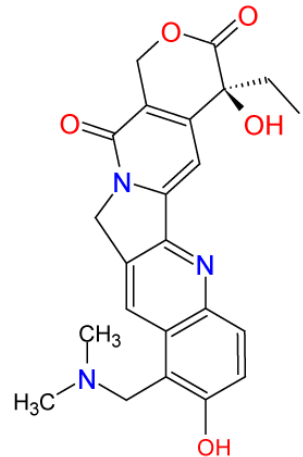

Irinotecan

Prodrug, inactive<smiles>CCc1c2c(nc3ccc(OC(=O)N4CCC(C)CC4)cc13)-c1cc3c(c(=O)n1C2)COC(=O)[C@@]3(O)CC</smiles><smiles>CCc1c2c(nc3ccc(O)cc13)-c1cc3c(c(=O)n1C2)COC(=O)[C@@]3(O)CC</smiles>

B
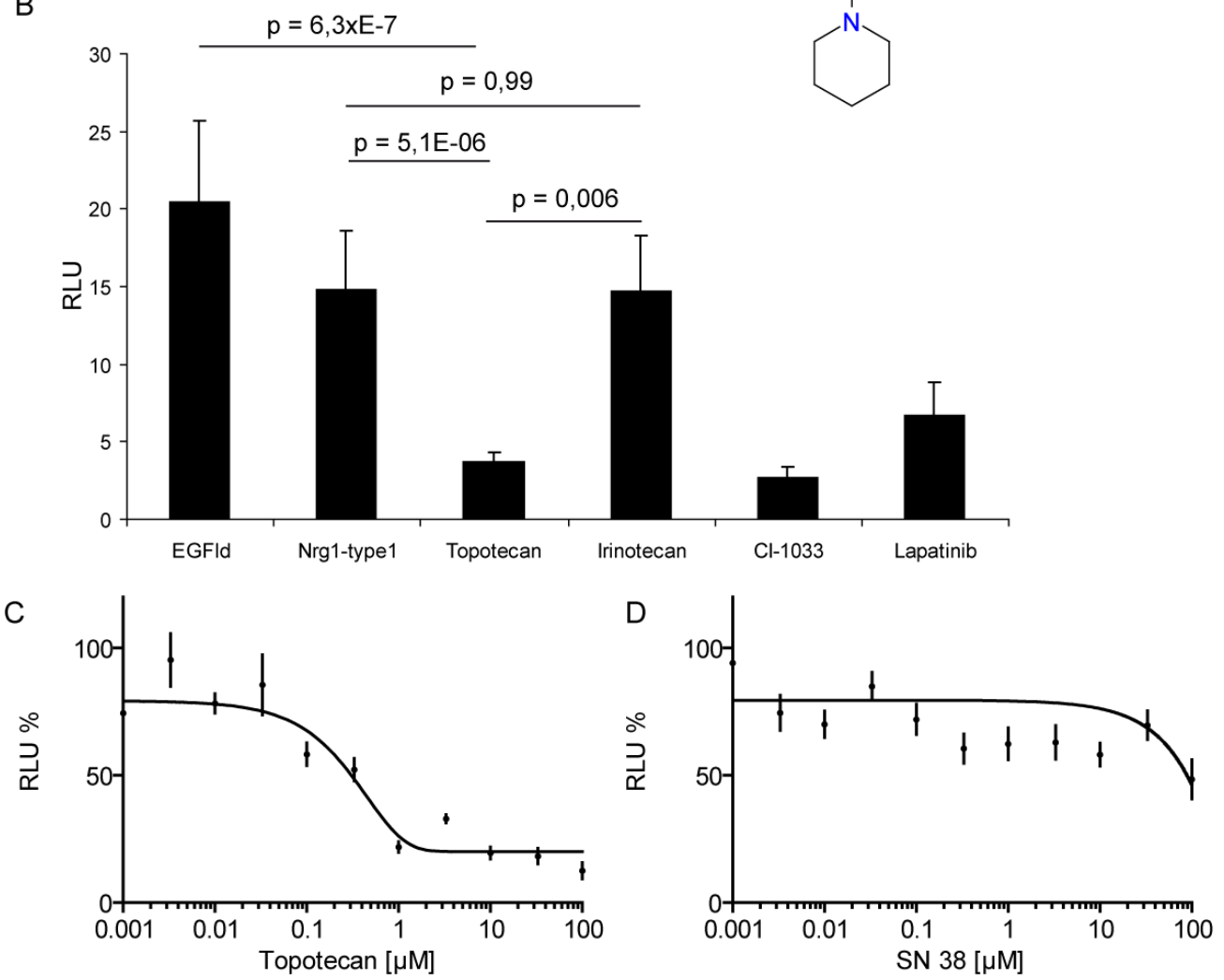

Figure 66: Effect of Topotecan derivatives Irinotecan and SN38 on the
ERBB4/PIK3R1 split TEV assay

A) Comparison of the chemical structures of Camptothecin derivates. Chemical structures of Camptothecin, Topotecan, Irinotecan, SN38. B) Data set from the NCC003 screen.

The difference between Topotecan and Irinotecan on the ERBB4/PIK3R1 co-culture screen is shown. C-D) Individual effects of Topotecan (C) and SN38 (D).

ERBB4 was fused to NTEV-tevS-GV-2HA; PIK3R1 was fused to CTEV-2HA. Cells were stimulated with $10 \mathrm{ng} / \mathrm{ml}$ EGFld. 
A

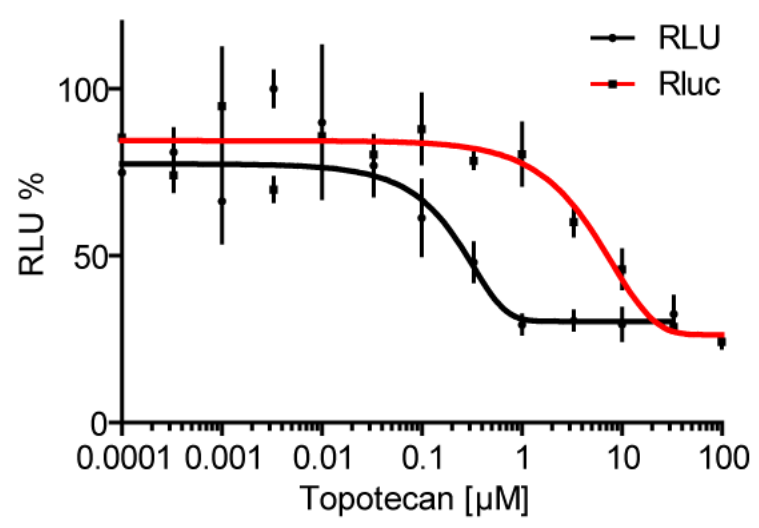

B

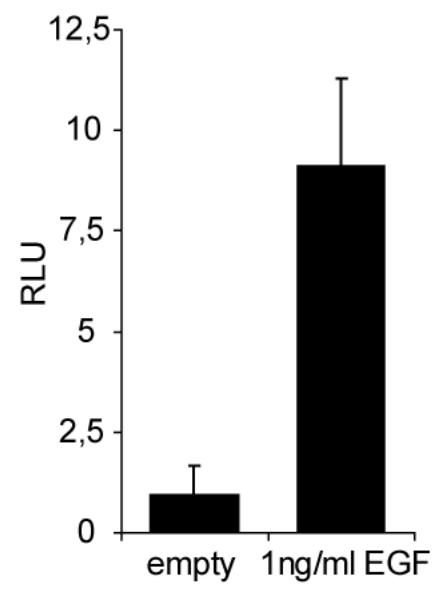

Figure 67: Effect of Topotecan on ERBB1/1 dimerisation induced by EGF

A) ERBB1 was fused to NTEV-tevS-GV-2HA; ERBB1 was fused to CTEV-2HA. PC12 cells were stimulated with $1 \mathrm{ng} / \mathrm{ml}$ EGF. B) Activation window of the EGF-induced ERBB1/1 assay. Setup as in (A).

A

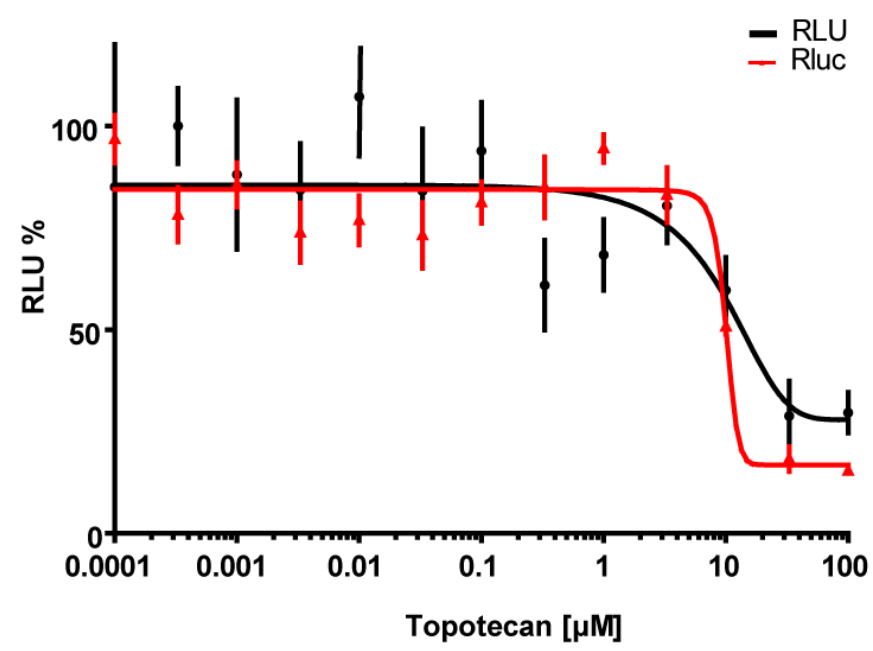

B

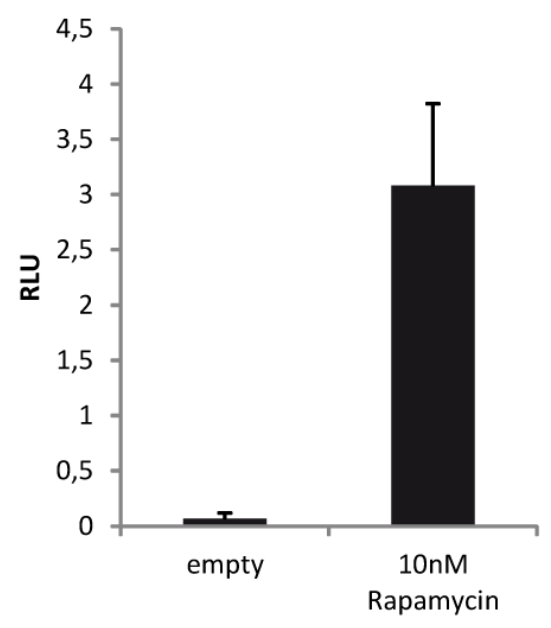

Figure 68: Effect of Topotecan on the FRB/FKBP interaction induced by Rapamycin A) TM-FRB was fused to NTEV-tevS-GV, FKBP was fused to CTEV. PC12 cells were stimulated with 10nM Rapamycin. B) Activation window of the Rapamycin-induced FRB/FKBP assay. Setup as in (A). 


\subsection{Validation Mevastatin}

Mevastatin (Compactin penicillium) is an antifungal metabolite from Penicillium breviocompactum. It is an HMG-CoA reductase inhibitor (cholesterol biosynthesis) with multiple side effects, which prevent the therapeutic usage.<smiles>CC[C@H](C)C(=O)O[C@H]1CCC=C2C=C[C@H](C)[C@H](CC[C@@H]3C[C@@H](O)CC(=O)O3)C21</smiles>

Figure 69: Chemical structure of Mevastatin

Mevastatin showed a significant activation of the firefly luciferase activity in the screen. In the ERBB4/PIK3R1 assay activated with 10 ng/ml EGF-like domain it showed a $250 \%$ activation of the relative luciferase activity at an $\mathrm{EC}_{50}$ of $0.7 \mu \mathrm{M}$.

\subsubsection{Technical controls for Mevastatin}

Renilla luciferase

In the Renilla luciferase assay Mevastatin showed a toxic effect wit an $\mathrm{IC}_{50}$ of $0.4 \mu \mathrm{M}$.

The comparison of both datasets indicate that the activation of the relative luciferase activity is reciprocal to the toxic effect of Mevastatin on the PC12 cells e.g. the Renilla luciferase activity.

Mevastatin has no specific effect on the ERBB4/PIK3R1 co culture assay. The Mevastatin dataset is an excellent example for the elimination of false positive hits from the screening results. 
A

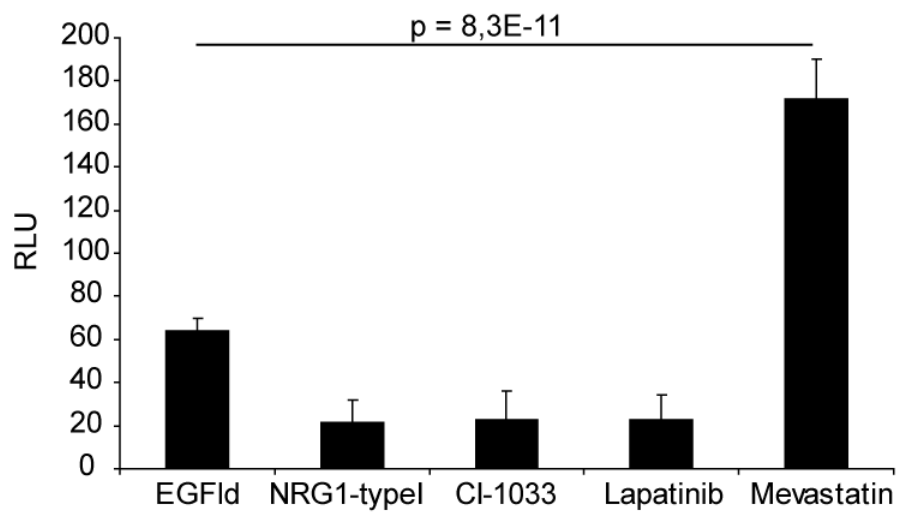

B

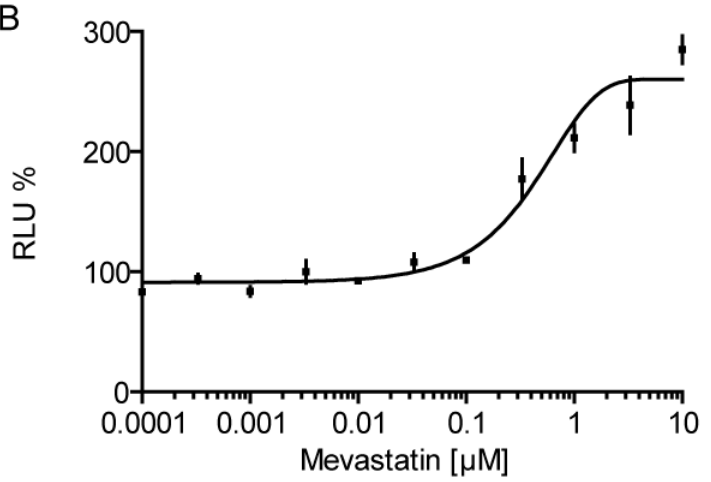

C

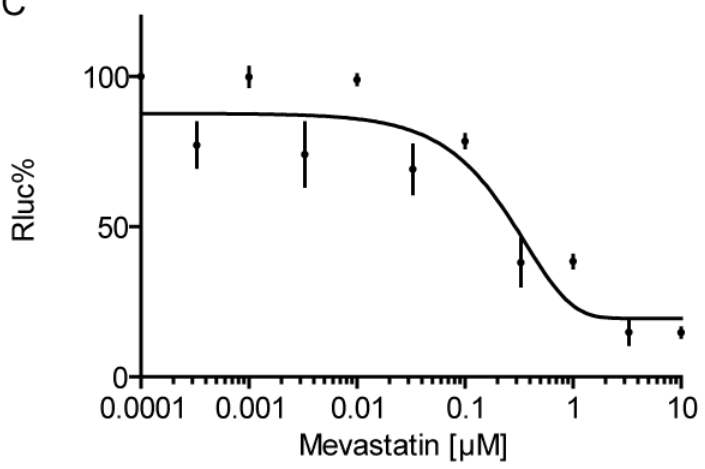

D

RLU+Rluc

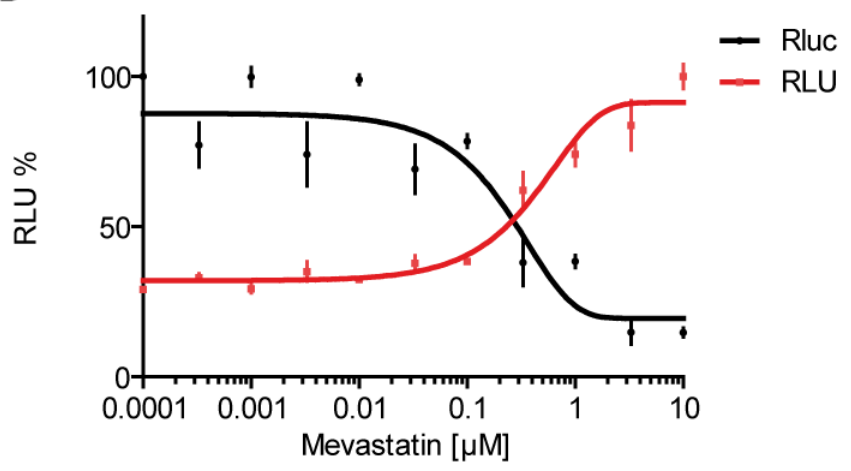

Figure 70: Validation Mevastatin

A) Data extracted from the screen. B) ERBB4/PIK3R1 $10 \mathrm{ng} / \mathrm{ml}$ EGFId. ERBB4 was fused to NTEV-tevS-GV-2HA; PIK3R1 was fused to CTEV-2HA. PC12 Cells were stimulated with $10 \mathrm{ng} / \mathrm{ml}$ EGFld. C) Renilla luciferase assay to test the toxicity of Mevastatin. D). Comparison ERBB4/PIK3R1 and Renilla luciferase, the increment of growth of the RLU value is dependent on the decrease in Rluc. 


\subsection{Validation CCPA}

2-chloro-N(6)-cyclopentyladenosine (CCPA) is a specific agonist for the Adenosin A1 receptor (Gao and Jacobson, 2002).

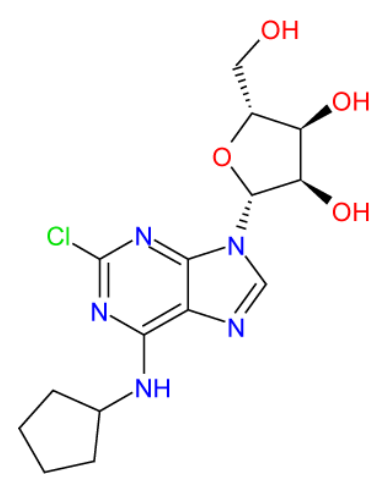

Figure 71: Chemical structure of CCPA

CCPA showed a significant activation of the firefly activity in a preliminary coculture screen witch did not reach a z'-factor of 0.5. CCPA showed a two fold activation of the firefly luciferase activity in the ERBB4/PIK3R1 co-culture assay.

\subsubsection{Technical controls for CCPA}

\section{Renilla luciferase}

CCPA showed no toxic effect on the Renilla luciferase.

\section{The Gal4-VP16 control assay}

In the technical vallidation CCPA showed an two fold activation of the firefly activity.

\section{TEV protease control assay}

In the TM-TEV/TM-GV assay CCPA showed a two fold activation of the firefly activity.

\subsubsection{Vertical validation}

CCPA showed although a two fold activation of ERBB4/SHC adapter recruitment and a two fold activation of ERBB4 homodimerisation.

As the technical controls indicate CCPA is not an activator of the NRG1ERBB4 system. It interfers unspecific with the firefly activity or cell activity, activating it 2 fold between a CCPA concentration of $0.01 \mu \mathrm{M}$ and $1 \mu \mathrm{M}$. 
A

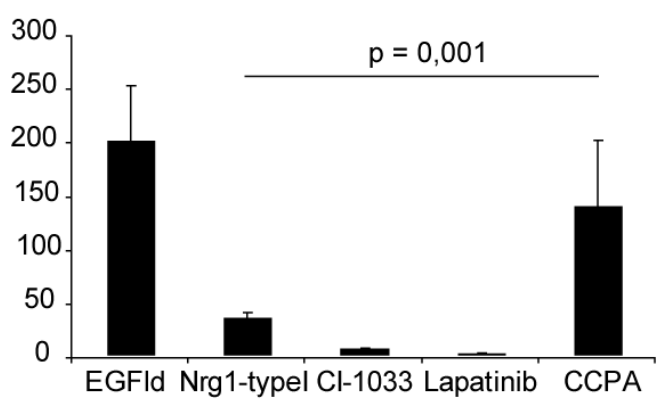

B ERBB4/PIK3R1 10k Nrg1-typel

C

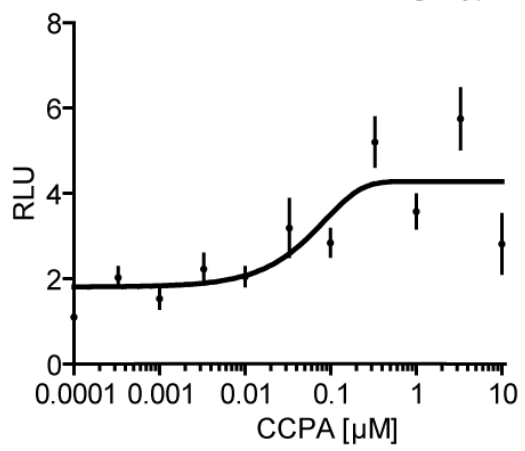

Rluc

GV

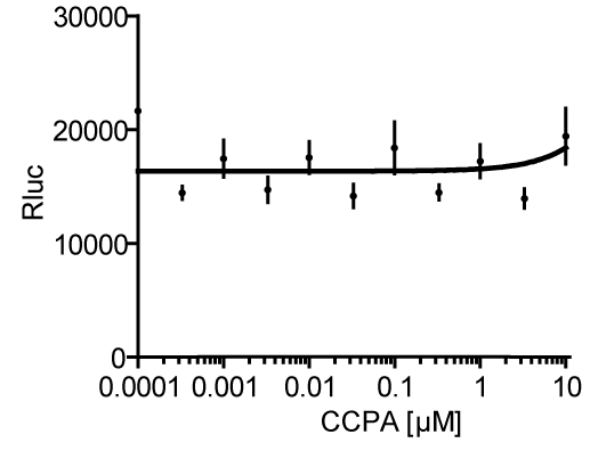

D

E

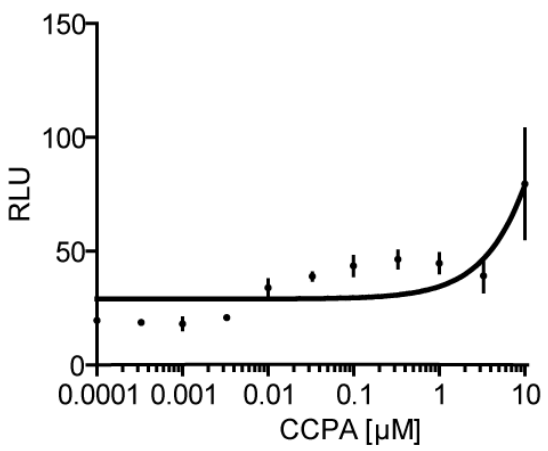

TM-TEV / TM-GV

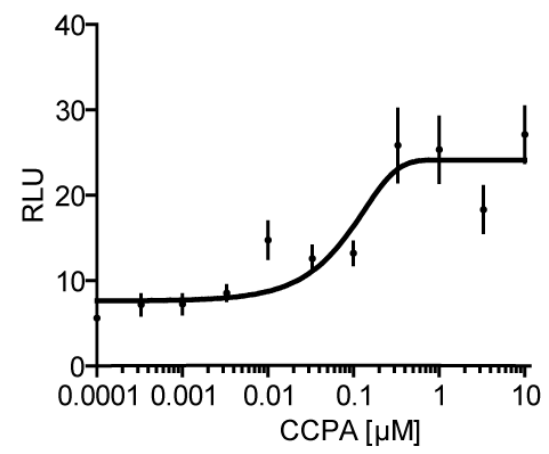

$\mathrm{F}$

ERBB4 / SHC

G

ERBB4 / ERBB4
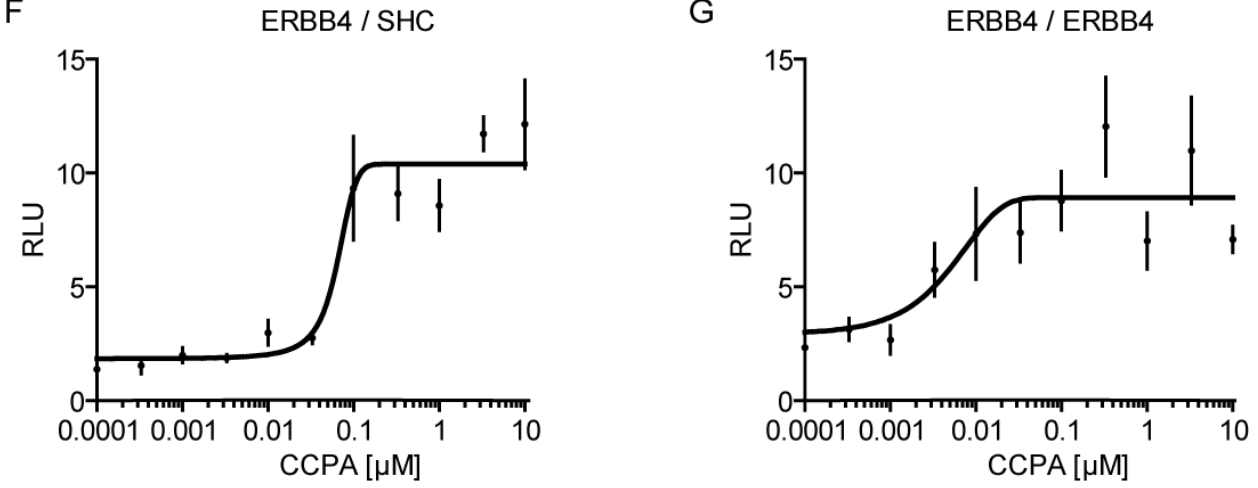

Figure 72: Validation CCPA

A) Extracted data from the screen. B) Co culture assay Nrg1-typel-ERBB4/PIK3R1. Split TEV assay in PC12 cells. ERBB4 was fused to NTEV-tevS-GV-2HA; PIK3R1 was fused to CTEV-2HA. C) Renilla luciferase assay to test the toxicity of CCPA. D) GV/ UAS-Fluc. E) TM-TEV/TM-GV.F) ERBB4/SHC split TEV assay in PC12 cells. ERBB4 was fused to NTEV-tevS-GV-2HA. SHC was fused to CTEV-2HA. Cells were stimulated with $10 \mathrm{ng} / \mathrm{ml}$ EGFId. G) ERBB4/ERBB4 split TEV assay in PC12 cells. ERBB4 was fused to NTEVtevS-GV-2HA. ERBB4 was fused to CTEV-2HA. Cells were stimulated with $10 \mathrm{ng} / \mathrm{ml}$ EGFId. 


\subsection{Validation Vincristine, effects on Renilla luciferase}

Vindesine and Vincristine are vinca alkaloid derivatives from Catharanthus roseus. (Madagaskar Evergreen). They are antimitotic chemotherapeutics used in the treatment of cancer and as immunosuppressive drugs. The akaloids interfere with the formation of tubulin into microtubules therby inhibiting cell division. Further effects are on RNA/DNA synthesis, lipid biosynthesis, cyclic nucleotide metabolism, glutathione metabolism and calmodulin-dependent $\mathrm{CA}^{2+}$-transport ATPase (Jordan et al., 1985).

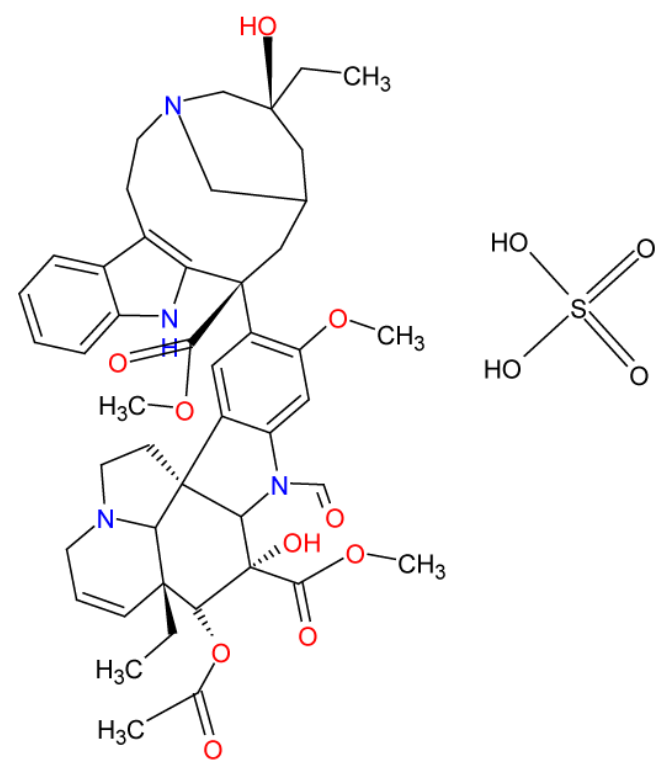

Figure 73: Chemical structure of Vincristine sulfat

Vindesine and vincristine showed a minor significant activation of the firefly activity $(p<0.009)$ in a preliminary co-culture screen that did not reach a $z^{\prime}-$ factor of 0.5. Vinchristine was further analysed and showed a 10 fold activation of the firefly luciferase activity between $0.001 \mu \mathrm{M}$ and $1 \mu \mathrm{M}$ in the complete ERBB4/PIK3R1 co-culture assay. It showed a high toxic effect on the Renilla luciferase assay at an $\mathrm{IC}_{50}$ of $0.015 \mu \mathrm{M}$ reducing Renilla luciferase activity. In the technical vallidation Vincristine showed a ten fold activation of the GV/UAS-Fluc assay and a ten fold activation of the TM-TEV/TM-GV assay. Both increases in the relative luciferase values are due to the $90 \%$ decrease of Renilla luciferase activity.

Vincristine showed although a ten fold activation of ERBB4/SHC and a ten fold activation of the ERBB4 homodimers.

As the technical controls indicate Vincristine is not an activator of the Nrg1typel-ERBB4 signalling system. It interfers with the Renilla activity, decreasing the Renilla activity with an $\mathrm{IC}_{50}$ of $0.015 \mu \mathrm{M}$. The observed activation is a pure 
mathematical artefact, resulting from the calculation of relative luciferase units (RLU).

A

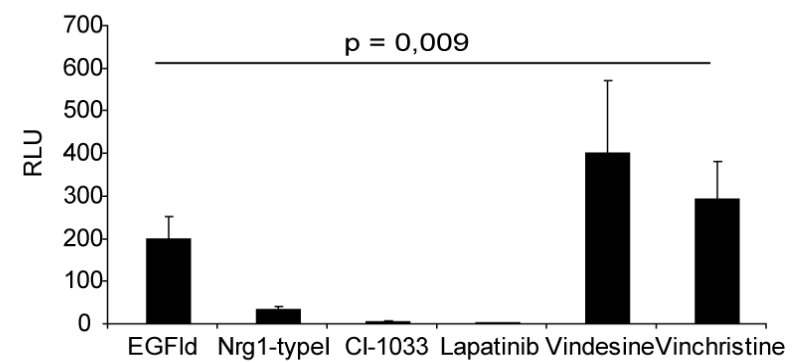

B

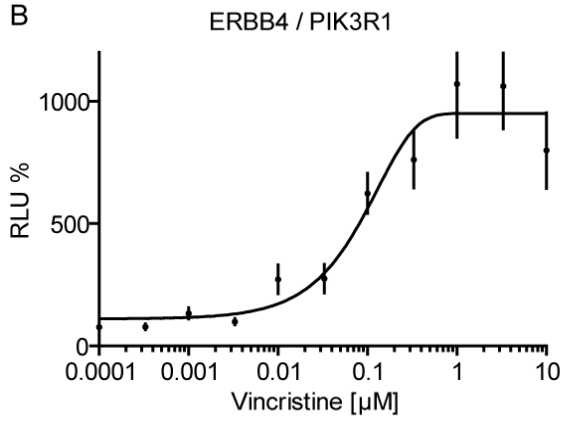

D

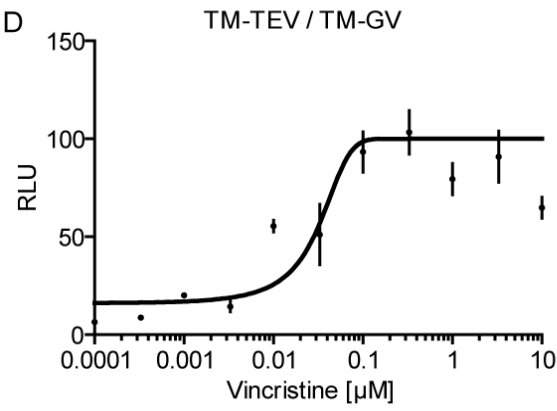

F

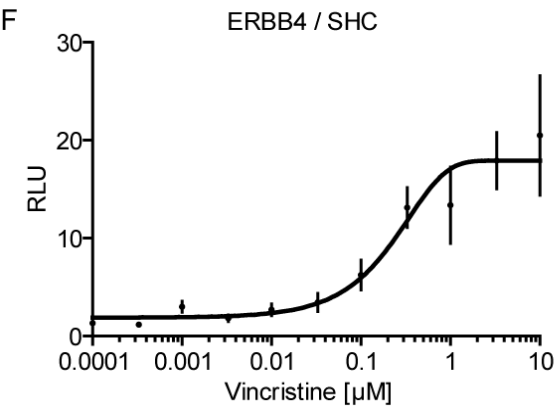

C

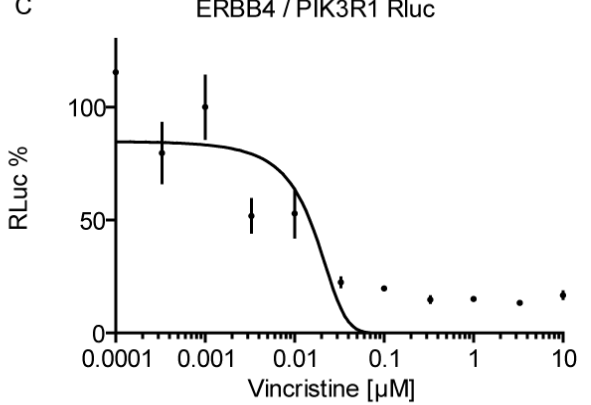

E

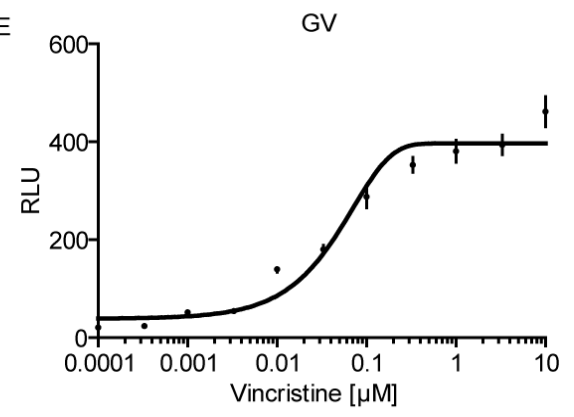

G

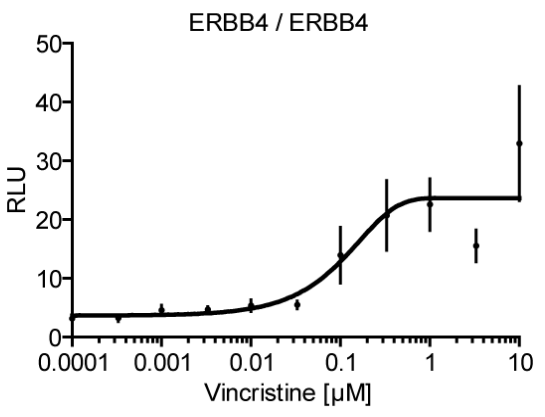

Figure 74: Validation Vincristine

A) Extracted data from the screen. B) Co culture assay Nrg1-typel-ERBB4/PIK3R. Split TEV assay in PC12 cells. ERBB4 was fused to NTEV-tevS-GV-2HA; PIK3R1 was fused to CTEV-2HA. C) Renilla luciferase assay to test the toxicity of Vincristine. D) GV/UAS-Fluc. E) TM-TEV/TM-GV. F) ERBB4/SHC split TEV assay in PC12 cells. ERBB4 was fused to NTEV-tevS-GV-2HA. SHC was fused to CTEV-2HA. Cells were stimulated with $10 \mathrm{ng} / \mathrm{ml}$ EGFld. G) ERBB4/ERBB4 split TEV assay in PC12 cells. ERBB4 was fused to NTEVtevS-GV-2HA. ERBB4 was fused to CTEV-2HA. Cells were stimulated with $10 \mathrm{ng} / \mathrm{ml}$ EGFId. 


\subsection{Validation K252a}

The Indolocarbazole K252a is an alkaloid from Nocardiopsis spec. and a Staurosporin analogue. It is a highly specific CaM kinase and phosphorylase inhibitor $\left(\mathrm{IC}_{50} 1.8\right.$ and $\left.1.7 \mathrm{nmol}\right)$ and a serine/threonine protein kinase inhibitor $\left(\mathrm{IC}_{50} 10\right.$ to $\left.30 \mathrm{nmol}\right)$ (Borasio, 1990).

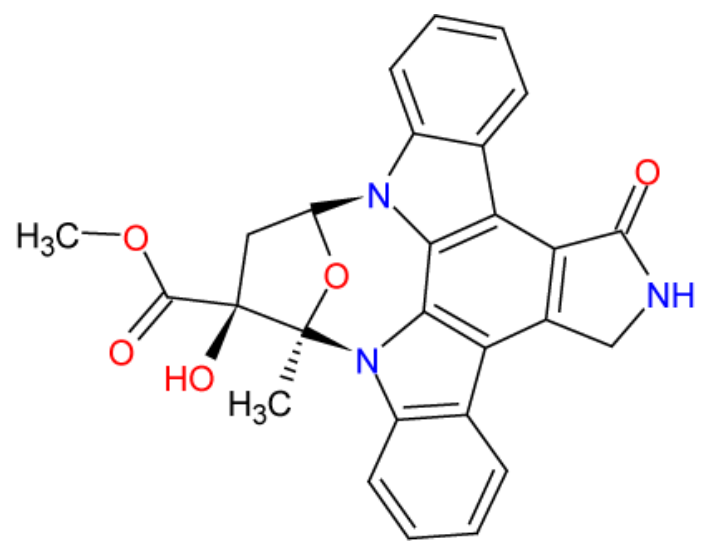

Figure 75: Chemical structure of K252a

K252a was described by Kuai (Kuai et al., 2010) to potentiate Nrg1 induced neuritogenesis in PC12 cells transfected with ERBB4. In addition, Kuai et al. showed that the effect of K252 depends on the potentiation of the localisation of ERBB4 receptors to the membrane and an elevation of ERBB4 receptors in the membrane due to altered endocytosis (Kuai et al., 2011). Therefore, I wished to test, whether K252a could activate NRG1-ERBB4 signalling using the split TEV ERBB4/PIK3R1 co-culture assay. However, K252a did not activate ERBB4 signalling, and proved only to be toxic for the cells.

Surprisingly, K252a showed an inhibition of the relative luciferase activity in the ERBB4/PIK3R1 co-culture assay, with an $\mathrm{IC}_{50}$ of $0.7 \mu \mathrm{M}$. The technical control Renilla luciferase showed an $\mathrm{IC}_{50}$ of $0.2 \mu \mathrm{M} \mathrm{K} 252 \mathrm{a}$. Thus, the reduction in relative luciferase activity was not caused by a specific effect on NRG1ERBB4/PIK3R1 signalling, but rather through a toxic effect elicited onto the cells. 
Results

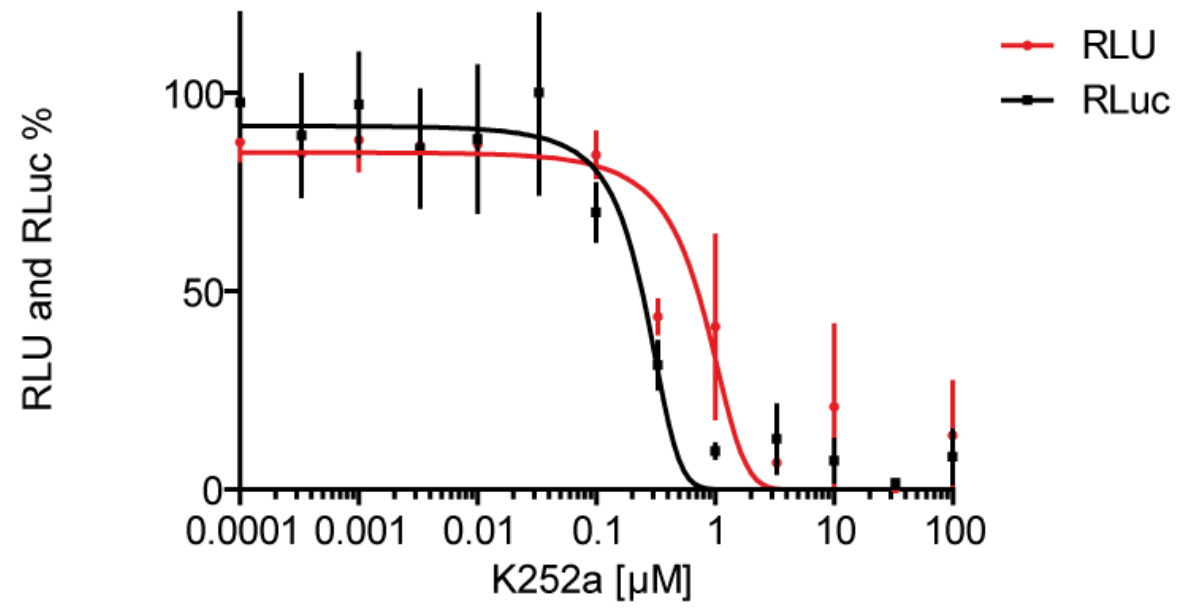

Figure 76: Validation of K252a

ERBB4/PIK3R1 10ng/ml EGFld. ERBB4/PIK3R1 split TEV assay in PC12 cells. ERBB4 was fused to NTEV-tevS-GV-2HA. PIK3R1 was fused to CTEV-2HA. Cells were stimulated with $10 \mathrm{ng} / \mathrm{ml}$ EGFld. Cells were harvested after $24 \mathrm{~h}$ and luciferase data was analysed using graph pad prism. $n=6$, error bars represent standard error of the mean. 


\section{Discussion}

\subsection{Technical issues of cell-based assays}

\subsubsection{Translational assays and using GWAS data to model SZ.}

Most of the findings gained from screening assays in animal models remain serendipitous. Animal models for SZ, which mimic the entire complexity of the disease, are not available for SZ (Nestler and Hyman, 2010). Therefore, the lack of construct validity in animal models favours simpler models, with one simple model covering one aspect/genetic trait of SZ. Those molecular isolated aspects of SZ may also be modelled in cell-based assays. Various pathways are known to be involved in SZ on a molecular level, which is consistent which the hypothesis that $S Z$ is also seen as an umbrella term for various diseases with similar disease patterns (Pratt et al., 2012; Keshavan et al., 2008; Marín, 2012).

Several genetic association studies revealed the relevance of malfunctions in the NRG1-ERBB4 signalling system for the development of (Stefansson et al., 2003; Stefánsson et al., 2003; Stefansson et al., 2002; Buonanno, 2010; Li et al., $2004 \mathrm{Lu}$ et al., 2010 Weickert et al., 2012). To address this signalling pathway in more detail, I designed a cell-based co-culture assay that could partially mimics the communication between two distinct neurons via Nrg1ERBB4. Hits resulting from this compound screen were shown to target the Nrg1-ERBB4 signalling pathway and could be used in the future to treat SZ mouse models to prove the hits' in vivo relevance.

\subsubsection{Rational design of the assay workflow}

Critical to compound screenings are the time points of measurements(Macarron et al., 2011; Ohlmeyer and Zhou, 2010). Large screens imply the usage of huge substance libraries. These libraries are expensive in maintenance and the costly and laborious handling and storage forces the user to test an individual compound in a primary screen only once. Therefore, only a compound's single effect of a single administration can be usually measured without exceeding available budgets.

This requires assays of highest quality and reliability. Moreover, the handling of the cells and the screening time points have to be adjusted further to get an optimal window for the measurements. This includes several technical issues, such as enzyme activity, instrument availability, and manpower. The applied assay is a transcriptional-based assay with the activity of the firefly luciferase as readout. Firstly, the transfected cells need time to express the components required for the cell-based assay. In our cell-based assay, the cells need $12 \mathrm{~h}$ 
to sufficiently express the components. This is especially important for the correct localisation of the ERBB4 receptor. Secondly, after stimulation, the cell needs $12 \mathrm{~h}$ time to express the reporter protein to get satisfying levels that can be measured. After testing various combinations and protocols, I decided to apply a $24 \mathrm{~h} / 24 \mathrm{~h}$ measurement cycle, i.e. the cells were allowed to express the assay components for $24 \mathrm{~h}$, then stimulated with compounds and controls, and $24 \mathrm{~h}$ later they were lysed for luciferase assay analysis. This implies a $12 \mathrm{~h} / 12 \mathrm{~h}$ measurement cycle. Also, measurement cycles are possible between $12 \mathrm{~h}-$ $12 \mathrm{~h}$ and $36 \mathrm{~h}-36 \mathrm{~h}$. To integrate the screen in daily working hours a $24 \mathrm{~h}-24 \mathrm{~h}$ setup is most preferable.

\subsubsection{Selection of constructs}

Reaching construct validity for SZ is an ambitious goal and is so far not possible. It might be partially possible in the future, when patient-derived induced pluripotent stem cells (iPSC) could be instructed to relevant neirnal lineages and using these cells e.g. in phenotypic screen (Brennand et al., 2011; Brennand and Gage, 2012; Brennand and Gage, 2011). The NRG1ERBB4 system signalling pathway itself is a proven target, but this signalling network is far more complex to be reflected used in one single assay (Mei and Xiong, 2008). NRG1 has a large number of isoforms (more than 30 are yet described). The isoforms differ in length, function, localisation, and processing properties (Fleck et al., 2011; Luo et al., 2011; Mei and Xiong, 2008; Meyer and Birchmeier, 1995).

ERBB4 consists of four major isoforms (JM-a, JM-b, Cyt1, and Cyt-2)(Zeng et al., 2007a; Yarden and Pines, 2012). To this end it is unknown, which exact isoform(s), has/have what kind of impact on SZ, and in which cell types this may be cause a specific part in SZ. Tremendous effort has been done by the GWAS studies. However, they cannot deliver this kind of information.

For the Nrg1-ERBB4 cell-based assay, I chose Nrg1.typel- $\beta 1$ a mainly for the reason of assay performance. The Nrg1-typel containing cells delivered a far better measurement window than the Nrg1-typelll (Figure 26). The most active part of all NRGs is the EGF like domain, which is encoded in NRG1typel- $\beta 1$ a but also in typelll, and in the corresponding murine versions.

For ERBB4 receptors, I chose the JM-a-Cyt1 variant, as it encodes a PI3Kbinding site that is necessary to integrate PI3K signalling given the importance of this downstream process in the context of SZ (Law et al., 2012). All adapters tested performed well when assaying for ERBB4 receptor activation. Importantly, the PI3K adapter protein PIK3R1 performed best, and was recently reported as a SZ target (Law et al., 2012). 
The subset of Nrg1-typel- $\beta 1$ a/ERBB4-JMa-Cyt1/PIK3R1 was mainly chosen to meet the needs of assay performance and not as neccessarily being the most relevant for SZ. Nonetheless, SNPs enhancing the expression of the ERBB4-JMa-Cyt1 variant seems to associated with an elevated risk of SZ (Silberberg et al., 2006; Law et al., 2007).

\subsubsection{Artificial and tagged proteins in the cell-based assay}

The ERBB4 receptor and the adapter proteins that get recruited to the activated ERBB4 are modified (i.e. tagged) to make them applicable to the split TEV assay technique. For the split TEV system, it has been shown that the TEV fragments do not affect the function of the proteins under investigation (Wehr et al., 2008). However, it cannot be ruled out that some chemical compounds target the TEV fragments. Therefore, false positive hits that could be directed against artificial components of the cell-based assay had to be removed during the secondary analysis.

\subsubsection{In vitro screens vs. co-culture screening systems}

To date, many large-scale screens are done in in-vitro assays (Macarron et al., 2011; Inglese et al., 2007). These screens deliver fast and specific binding data for substances that perform excellent against a single target. However, the absence of the biological context results in high attrition rates for compounds identified in in-vitro binding assays due to unanticipated off-target effects that are detected in later phases. For example, off-target effects can be caused by unspecific activation of non-desired signalling pathways that act opposite to the desired effects.

Cell-based screens allow the integration of various signalling pathways into an overall cellular response, and toxic side effects and/or effects on downstream targets may be immediately measured (Lievens et al., 2012). However, limitations of cell-based assays are that usually only one or two different assay aspects can be addressed within a screen. Multiplexed cell-based assays, at least starting from the stage of hit-to-lead optimisation and later, may prove invaluable to increase information collected for a given compound. Therefore, the luciferase reporter has already been replaced successfully with encoded molecular barcodes that principally would allow monitoring of several cellulat parameters in parallel (Botvinnik et al., 2010).

Cell-based screens utilising engineered cell lines or transiently transfected cells may prove equally well in screens. However, overexpressing proteins and alterations thereof to match the requirements of the cell-based assay have to be carefully tested to avoid any artificial effects. 
The presented co-culture assays represent a technological progress over standard cell-based assays based on a single cell poulation. Such an assay is specifically designed to monitor the communication between two populations of cells and can monitor inter- as well as intracellular signalling (Wehr et al., 2008a). These cells may be of different origin, where the first population may represent cells producing a signal-emitting ligand, and the second population may express a receptor that receives the signal. Ligands like NRG1 have to be expressed, processed, correctly localised and integrated into the plasma membrane, or even secreted to be fully functional (Falls, 2003). Likewise, the ERBB4 receptor can only signal correctly when specifically activated at the plasma membrane (Yarden and Pines, 2012). A co-culture assay using, firstly, a Nrg1-expressing and, secondly, an ERBB4-expressing cell population guarantees that the ERBB4 receptor is only activated at the plasma membrane, thereby best mimicking the natural situation.

\subsubsection{Limitations of co-culture systems vs. animal models}

Results obtained from cell-based assays, including co-culture assays, may have different degrees of implications for translational experiments planned thereafter in animal models. This is particularly true for cell-based assays designed to address neurobiological relevant mechanisms.

In this compound screen, I have used PC12 cells, which are pheochromocytoma cells isolated from the rat adrenal medulla (Greene and Tischler, 1976). Therefore, these cells are neither neurons, nor organised in circuits, layers or tissues, and were not cultivated in a 3D format. PC12 cells were grown in flat-bottom 96-well plates for the assays performed. Compounds tested were pipetted onto the cells, and did not have to cross the blood-brain barrier, and compounds were also not degraded by liver functions. Nonetheless, compared to the hetelogous cell lines usually engaged for HTS campaigns (such as HEK293 or CHOk1 cell lines), PC12 cells seem to be more appropriate in a neurobiological context since closely related to the sympathoadrenergic lineage of neurons.

Conversely, a given mouse model is a compromise between the very complex human brain and a rather simple cell-based co-culture assay. In addition, a mouse brain lacks several key features found in a human brain. This is particularly true for the ability to communicate in an abstract manner. For example, abnormal communication with other humans has been widely reported in SZ patients, and this phenotype can only be partially mimicked in mice, using the Open Field and other related tests (Pratt et al., 2012).

Nevertheless, a well-developed cell-based assay may provide promising initial data that can be further corroborated using a suitable animal model. This 
model, in turn, may offer opportunities to conclude signalling and wiring functions up to the human situation.

\subsubsection{Screening of compound libraries: The hit-to-lead process in drug discovery}

When screening large compound libraries, hit compounds are most likely clustered into sets of similar substances. These hits have to be confirmed using alternative assay. To enter the next phase, i.e. the hit-to-lead process, the isolated compounds have to be modified using medicinal chemistry, and the following lead structures will be analysed and further optimised towards improved signalling characteristics. Therefore, in a prospective manner, greatest care was taken to develop and test several secondary validation assays for robustness and fast applicability (see below).

\subsubsection{Screening of FDA approved drug libraries}

The NCC library is a collection of FDA-approved drugs that have a history of clinical usage, and important information such as toxicity, bioavailability, side effects etc. are well described. In addition, the safety, best way of application, and maximum dosage for humans is already known for the respective application.

Therefore, using the NCC library is different from the screening of other compound libraries as putative hits are already FDA-approved drugs, which implies that a given hit could potentially be immediately used in patients. However, many drugs have a spectrum of side effects indicating that they have more targets than the one they were primarily designed for. A simple and straightforward approach is to test known drugs for yet undiscovered therapeutic potential. The NCC library was exactly designed for this purpose. All drugs in this library are well described and can be further tested investing little time and effort. However, as these drugs are already on the market patent issues may prove complicated when a given drug from this library will be applied in a new context. Pharmaceutical companies have no interest to reassess patented drugs in new clinical trials. A solution to this could be a non-profit, government-financed consortium that takes over responsibilities.

\subsubsection{Transfer of the HTP screen to the LDC}

Problems in assay miniaturisation from a 96-well to a 384-well format

For HTP screens of large libraries, such as for the library from LDC comprising more than 200,000 compounds, a miniaturisation of the assay is needed to optimise handling. For example, amounts of valuable drug portions should be reduced, and the number of data points per handling unit, such as a 
cell culture plate, should be increased, also allowing for the generation of more data sets in less time.

The assay presented in this thesis worked well in the 96-well format, with 40,000 assay cells per well and 10,000 Nrg1-typel cells added, with a z' factor of $>0.5$ indicating a good separation window for the screen. Attempts to miniaturise the assay to a 384-well format caused problems in the assay's stability, as indicated by a rather low z' factor of around 0.3 . Initial tests to simply downsize each component by a factor of four did not prove helpful. Colleagues at the LDC are now devoting much of their time and energy to get the assay working. Currently, it is expected that the large compound screen will be performed in a single cell assay including a stimulation using the EGFlike domain. For secondary screening, however, it is planned to use the coculture assay in a 96-well format.

\subsubsection{Elimination of false positives}

Firefly luciferase activity was used as readout in the screen, and hits were scored for increased or reduced readings. A given drug may alter these readings by influencing the viability of the assay cells or by acting on the reporter proteins themselves, e.g. by changing their expression, efficient folding and sorting, or localisation, thereby leading to the identification of a false positive hit (Sink et al., 2010). For example, a given drug may promote or inhibit the firefly luciferase activity (Cheng and Inglese, 2012), or impinge on the correct functionality of the artificial co-transcriptional activator GV or the TEV protease. To eliminate these false positive hits, we implemented several measures in the screen itself and during the secondary screening process. For the screen, a second luciferase, the Renilla luciferase under the control of the constitutive TK-promoter was used to assay for viability. Therefore, all highly toxic substances can be excluded from the analysis as they strongly reduce Renilla readings. During the process of secondary screening, further tests were run to address a given drug's effect related to the functionality of GV, the TEV protease, and the concomitant release of GV induced by TEV activity. To do this, I re-screened the entire library using (1) a setup of constitutive GV expression and the firefly luciferase reporter UASFluc and (2) a membrane-anchored TEV protease, a membrane-anchored GV, and the reporter UAS-Fluc. In summary, applying these controls helped to eliminate false positives.

\subsubsection{Elimination of false negatives}

The elimination of false negatives is less straight forward. For example, a given drug that has an effect on ERBB4 but does not score in the chosen cellbased assay because of the chosen assay time point. For example, long-term effects of drugs cannot be monitored at the $24 \mathrm{~h}$ time point, whereas 
immediate and short-term effects may have already faded away. Each screen that we performed, however, was run in replicates, and complex statistical processing contributes to minimise false negatives. However, if the cell-based assay does not respond to a treatment, either due to a chosen readout or a given sensitivity, a selected assay time point, or a host cell, a potentially potent drug will not be recovered.

\subsubsection{Reproducibility of screening results}

The split TEV technique is a highly robust and sensitive method for cell-based assays (Capdevila-Nortes et al., 2012; Wehr et al., 2006; Wehr et al., 2008a, Djannatian et al., 2011) and was recently successfully used in an RNAi screen (Michael Wehr, personal communication). Here, the split TEV technique was applied for the first time in a compound screen, using the FDA-approved NCC library as template. For internal reasons, the NCC library was screened several times, i.e. to optimise protocols related to robotics and operational sequences. The library subset NCC201 was screened ten times in total, and Spironolactone was recovered each time as inhibitor of ERBB4 signalling. Other hits followed up upon in secondary screening, like Vincristin/Vindesine and CCPA scored in initial screens only, likely due to the setup process required to make the robotics unit fully functional (see table 16. for details).

\begin{tabular}{|l|l|l|l|}
\hline Name of drug & NCC subset & $\begin{array}{l}\text { Total times } \\
\text { recovered }\end{array}$ & State of validation \\
\hline Spironolactone & $201-1-\mathrm{D} 06$ & 10 & animal model \\
\hline Topotecan & $003-3-\mathrm{A} 04$ & 5 & stopped \\
\hline Vincristin & $003-6-\mathrm{B} 02$ & 1 & toxic \\
\hline CCPA & $003-6-\mathrm{B} 06$ & 1 & unspecific \\
\hline Albendazole & $201-3-\mathrm{A} 03$ & 2 & unspecific \\
\hline Mevastatin & $003-6-\mathrm{H} 04$ & 1 & toxic \\
\hline
\end{tabular}

Table 16. Secondary screening and validated hits

\subsubsection{Binding assays and co-immunoprecipitations}

Drugs recovered from the screen need to be validated using alternative assays. For example, drug-protein binding assays are necessary to verify that a drug physically associates with its target protein. Various protein-drug binding assays like isothermal titration calorimetry, surface plasmon resonace system, plasma protein binding assays, X-ray christallography and nuclear magnetic resonance are available (Arkin and Wells, 2004).

For the precise determination of protein-drug binding crystal structures and $X$ ray crystallography or nuclear magnetic resonance can be used. As both 
techniques are time-consuming an analysis using these methods is beyond the scope of this thesis. The following techniques are less time consuming. Surface plasmon resonance SPR (Huber, 2005) is a technique for the measurement of binding interactions. A surface bound immobilized molecule is probed against an analytic. Meassured is the change in refractive index of the surface after binding.

Isothermal titration calorimetry is a physical technique (Freyer and Lewis, 2008). Binding of small molecules to proteins can be measured using changes in Gibbs free energy and in entropy (Holdgate et al., 2010). This method has been used successfully for. ERBB Antibody binding was in addition profen by ITC (Castoldi et al., 2012).

The overexpression and purification of large membrane proteins, such as the ERBB4 receptor, however, is difficult. To study ERBB4-drug binding affinities using these methods, the receptor may be separated into various functional subunits, such as the cytosolic, a transmembrane, and an extra-cellular part, thereby circumventing purification problems.

In addition, the split TEV protein-protein interaction assay has to be complemented, i.e. by using a co-immunoprecipitation (co-IP) or a GST pulldown assay. Currently, ERBB4 and PIK3R1 are cloned into co-IP compatible vectors, and these experiments will commence shortly.

\subsubsection{Testing compounds on unrelated targets}

To assess target specificity of the compounds, unrelated but also closely related targets were chosen using the same cell-based assay format. To ensure the specificity of the hit compounds and to get a broader understanding of additional effects a spectrum of selected PPI was tested. The selection contained Rapamycin induced FKBP-FRB (test of general effect on induced PPI), and a GPCR mediated interaction with $\beta$-Arrestin2 (Serotonin 5a Receptor). Further tests like NTRK1 (also known as TRKA)SHC (to study an effect on another receptor tyrosine kinase) and further kinases are scheduled to increase informations about target specificity. 


\subsection{Relevance for schizophrenia}

The potential applicability of the candidates identified from NRG1-ERBB4 screens for treatment of $S Z$ is unclear. NRG1-ERBB4 signalling plays a key role in the development of the brain, and this process takes place over decades (Mei and Xiong, 2008). Both, in the screen and in the dose response validation experiments end point effects are measured $24 \mathrm{~h}$ after stimulation. It is unclear whether such an effect has an influence on brain architecture after many years post development. Nonetheless, each drug may be applied daily over long periods of time and may be functional even when half-life and ontarget effects are limited. Nonetheless, at least Spironolactone, which has a favourable tox-profile and is used in the clinic, might be applied in limited treatment trials at some point in patients. Before this might ever take place, several follow-up validations are essential. Most importantly, additional behavioral analyses with appropriate animal models need to be performed in the future, addressing working memory and social interaction tests for example.

SZ is regularly diagnosed after puberty or in early adulthood. The most significant changes in brain architecture (early years and puberty) are occurring at this time point. Finding and then also applying a drug that affects brain or neuronal circuit architecture during the human childhood and puberty raises major questions. First, it is ethically inconceivable to treat a child with brain structure-modifying drugs to prevent the relatively small (1\%) chance of contracting with SZ. Second, the severe side effects and unpredictable changes in the wiring of the brain do strictly bar this. In addition, to start a promising treatment scheme, a reliable biomarker that is present in children is needed, and a systematic analysis of a child's brain wiring has to be initiated as well. However, as such a biomarker is still elusive, treating children in this perspective remains far in the future.

Recent research shows (Nitsche et al., 2012; Lewis and González-Burgos, 2008) that the neuroplasticity and the on-going change in brain architecture exhibit sufficient neuroplasticity to be a putative target for the effects induced by drugs. Hopefully, drugs modifying NRG1-ERBB4 signalling will help to specifically change the neuronal circuit of inhibitory chandelier and basket cells and activating principal neurons in adults, thus re-balancing the initially disturbed circuit architecture. However, this question cannot be answered without studies in humans.

For the treatment of SZ, a moderate activator of NRG1-ERBB4 signalling is highly desired. In schizophrenia, reduced or potentially enhanced NRG1ERBB4 signalling (Weickert et al., 2012) leads to a loss of inhibition mediated by parvalbumin positive basket and chandelier cells ( $P V+$ interneurons) on 
principal pyramidal neurons, and an activator of NRG1-ERBB4 signalling could potentially alleviate symptoms related to this circuit architecture (Lewis and González-Burgos, 2008). However, no drug from the NCC library showed a specific activating effect on NRG1-ERBB4 signalling. Therefore, it is planned to use larger libraries of compounds to increase the chance to find an activator. For example, the analysis of the Spectrum collection (2320 compounds (http://www.msdiscovery.com/spectrum.html) was successful used by (Kocisko et al., 2003; Weissmann and Aguzzi, 2005; Sun et al., 2006; Weisman et al., 2006; Stallings-Mann et al., 2006). Moreover, the 200,000 compound library from the Lead Discovery Centre Dortmund is in progress (Eitner and Koch, 2009). 


\subsection{NRG1-ERBB4 - Spironolactone}

The inhibitory effect of Spironolactone was several times recovered in the screen. In addition, dose-response assays for Spironolactone validated a clear concentration-dependent inhibition of NRG1-ERBB4 signalling. Spironolactone was originally described as an antagonist of the mineralocorticoid receptor MCR (Rogerson et al., 2003). MCR regulates salt homeostasis in the kidney (Brewster and Perazella, 2004), and Spironolactone is used as a diuretic in the clinics (Ogden et al., 1961). MCR plays also a role in the brain (in particular in the hippocampus)(Oyamada et al., 2008), heart vasculature (Oyamada et al., 2008), and adipose tissue (Marzolla et al., 2012). Mechanistically, Spironolactone binds to the aldosterone binding pocket of the MCR, thus preventing its translocation to the nucleus, where the MCR induces the transcription of target genes (Grossmann et al., 2012)

For Spironolactone's action on NRG1-ERBB4 signalling, I speculate that Spironolactone could prevent ERBB4 receptors from dimerising thus reducing downstream signalling, i.e. preventing activation of downstream kinases, such as PI3K and AKT. Importantly, treatment of Spironolactone showed reduced phosphorylation levels of pTyr1284 in ERBB4, concomitant with a mild decrease in phospho-AKT levels. However, this signalling needs to be addressed in more detail in the future, including other SZ-relevant kinases, such as GSK-3 $\beta$ (Emamian, 2012).

\subsubsection{Does Spironolactone physically bind to ERBB4 receptors?}

Spironolactone specifically inhibits NRG1-ERBB4 signalling. However, split TEV assays do not allow answering whether Spironolactone physically binds to ERBB4 to inhibit receptor activation. Other assays discussed above are necessary to address this question.

\subsubsection{Spironolactone derivates and metabolites}

The secomd generation drug of Spironolactone Eplerenone showed no effect on ERBB4/PIK3R1 co-culture assay. The metabolite Canterenone showed also no effect.

This leads to the assumption that the thioketone (Acetylthio) group of Spironolactone is part of the mode of action. The entire description of the structure function relationship is beyond this thesis.

The penetration of the blood-brain barrier (BBB) is vital for any drug used for psychiatric treatment. Until now it is not known if Spironolactone passes the BBB (Geerling and Loewy, 2009). 


\subsubsection{Preliminary validation in a mouse model}

An analysis of Spironolactone effects counteracting MK801 induced psychosis (see MK801 mouse model) showed that Spironolactone shows only a nonsignificant tendency to reduce spontaneous locomotor activity. The administered Spironolactone concentration was $20 \mathrm{mg} / \mathrm{kg}$, relatively high compared to concentrations in humans $(20-200 \mathrm{mg} / 75 \mathrm{~kg}$ (average human)). As the effects are measured in two hours, they are supposably not based on Spironolactone's primary action against MCR and the resulting changes in gene expression (48h).

The MK801 mouse model is not the perfect model to validate putative NRG1ERBB4 inhibitors. A mouse model constitutively overexpressing Nrg1-typelll is in the validation phase and will soon be available ((Velanac et al., 2012) personal communication M. Schwab).

The effect of Spironolactone on the mouse brain has also to be shown in Western-Blots on mouse brain lysates and will be performed as soon as the model is available. 


\subsection{Topotecan shows strong toxic effects}

Topotecan is a semisynthetic variant of Camptothecin. Camptothecin is a pentacycline quinolone alkaloid found in the bark seeds, and fruits of the Chinese happy tree camptotheca acuminate (Xi Shu). Parts of the tree were regularly used in the treatment of different diseases in traditional Chinese medicine wheras no reports for the use in psychiatric treatments are available. In a screen for cancer therapeutics performed in 1966 by M. E. Wall and M. C. Wani camptothecin was isolated. It is not water-soluble and has severe side effects including unpredictable toxicity. Camptothecin acts as a topoisomerase I inhibitor (O'Leary and Muggia, 1998b; Redinbo et al., 1998; Morton, 1968).

Topoisomerases are enzymes that are localised to the nucleus, and are responsible for the relaxation of positive and negative supercoiled DNA strands. Camptothecin inhibits the breakage re-joining reaction of DNAtopoisomerase I. The inhibition is specific and results in the accumulation of reversible intermediate DNA-Camptothecin-topoisomerase I ternary complexes. Camptothecin only binds to the DNA-topoisomerase I complex, but not to DNA or the enzyme alone. (Gromova 1993, Hsiang 1989) (Gromova et al., 1993; Hsiang et al., 1989)

Topotecan is made water-soluble by a basic chain at carbon 9 . It is used against small lung cancer and ovarial cancers. It acts as Topoisomerase I inhibitor by stalling the DNA-topoisomerase I complex, and therefore acts cytostatic. For Topotecan, the penetration of the blood brain barrier, the localisation to the CNS, and cerebrospinal fluid concentrations of $32 \%$ in serum levels have been reported (Takimoto and Arbuck, 1997b)

Irinotecan (FDA-approved in 1998) is a pro-drug and undergoes hydrolysis by carboxylesterase, primarily in the liver. This conversion results is SN-38, which is the biologically active form of Irinotecan, with an increased activity of 1000-fold (Kawato et al., 1991; Redinbo et al., 1998; Kuhn, 1998; Zamboni et al., 1998). Irinotecan is used in the clinics to treat colorectal cancer.

Irinotecan has severe side effects including diarrhoea associated with abdominal cramping, vomiting, flushing, and diaphoresis. This is mediated by increased cholinergic activity, and this is reversible with atropine (Abigerges et al., 1995; Rowinsky et al., 1994). 

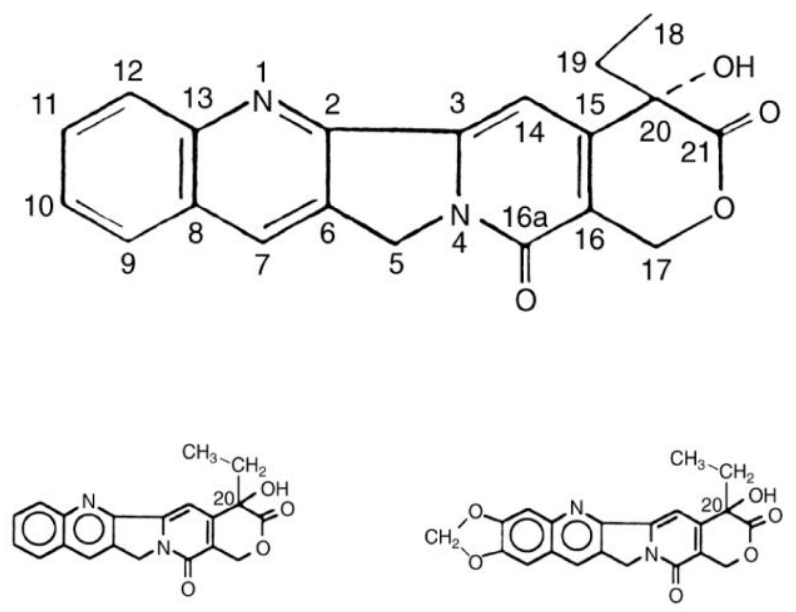

Camptothecin

10,11 -methylenedioxy-camptothecin
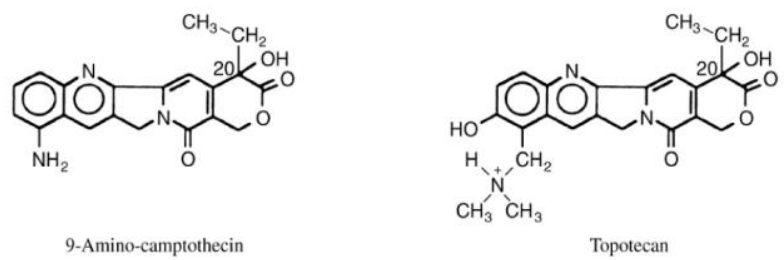

9-Amino-camptothecin

Topotecan

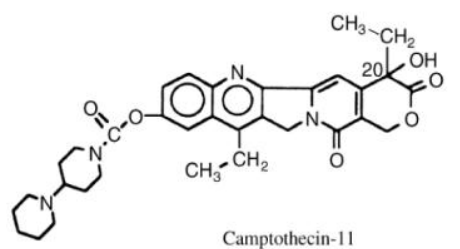

Figure 77: Immage. Campothecin derivatives (O'Leary and Muggia, 1998)

Camptothecins are toxic to the bone marrow and cause mucositis and diarrhoea (Irinotecan) in humans (O'Leary and Muggia, 1998a). They are only used as chemotherapeutics in live-threatening cancers (Taguchi et al., 1990). Therefore, usage of Topotecan in psychiatric patients is not recommended unless a lead optimisation processes results in lead structure with reduced toxicity i.e. reduced DNA-Topoisomerase I binding. On a chemical basis, it has already shown that binding of an amino group at $\mathrm{C} 12$ abolishes topoisomerase I inhibiting function and that specific stereochemistry at C20 is necessary. Only the $(S)$ isomer is active, racemic mixture shows $50 \%$ activity and the $(\mathrm{R})$ isomer is completely inactive (O'Leary and Muggia, 1998a). This combined with the inactivity of SN 38 may serve as template for a putative starting point of a leadoptimisation process, given the modified compounds remain inhibitory to NRG1-ERBB4 signalling.

Topotecan shows nicely the dilemma of the used co-culture assay systems. The time dimensions of an effect cannot be measured directly. Topotecan is strongly toxic for all fast-dividing cells, but the effect is not prominent in a $24 \mathrm{~h}$ assay. Toxic effects can accumulate and strike over time and remain hidden in short termed assays. 


\subsection{Conclusion}

Activators of NRG1-ERBB4 signalling are highly desired to compensate for SZ-induced loss of function phenotypes but remain elusive. The NCC library containing FDA-approved compounds was applied to address this issue. However, with Spironolactone and Topotecan, only inhibitors of NRG1ERBB4, were identified. As the NCC library only contains not far more than 700 drugs, larger collections, such as the Spectrum Collection containing 2300 compounds, will be used in the in the future. In addition, the LDC library, with more than 200,000 compounds, will be used to screen for activators of NRG1-ERBB4 signalling. Once an activator is identified, medicinal chemistry will be applied to generate new molecular entities in a lead optimisation process, with the aim to find a structure that activates NRG1-ERBB4 signalling, is not toxic, and fulfils the requirements to proceed into the preclinical stage of drug testing. 


\section{Abbreviations}

\begin{tabular}{|c|c|}
\hline BACE & Beta-site APP cleaving enzyme \\
\hline BRET & Bioluminescence resonance energy transfer \\
\hline BBB & Blood-brain-barrier \\
\hline Chr & Chromosome \\
\hline CIP & Calf intestinal alkaline phosphatase \\
\hline CMV & Cytomegalovirus \\
\hline CNS & Central nervous system \\
\hline CNV & Copy number variations \\
\hline CO-IP & Immunoprecipitation \\
\hline CRD & Cystein rich domain \\
\hline DISC1 & Disroupted in schizophrenia \\
\hline DMSO & Dimethylsulfoxide \\
\hline DNA & Desoxyribonuclein acid \\
\hline DSM IV & Diagnostic and Statistical Manual of Mental Disorders \\
\hline DTT & Dithiothreitol \\
\hline e.g. & For example/exempli gratia \\
\hline ECL & Enhanced chemiluminescens \\
\hline EDTA & Ethylenediaminetetraacetic acid \\
\hline EGF & Epidermal growth factor \\
\hline EGFP & Enhanced green fluorescent protein \\
\hline ERBB4 & $\begin{array}{l}\text { Receptor tyrosine-proteine kinase v-erb-a } \\
\text { erythroblastic leukemia viral oncogene homolog } 4 \text { (avian) }\end{array}$ \\
\hline ERT2 & Mutated Estrogen receptor domains \\
\hline f.c. & Final concentration \\
\hline FDA & Food and Drug Administeriation \\
\hline FRET & Fluorescence Resonance Energy Transfer \\
\hline GABA & Gamma-Aminobutyric acid \\
\hline GAD67 & Glutamat acid decarboxylase 67kd \\
\hline GRB2 & Growth factor receptor bound protein 2 \\
\hline GV & Galactose4.viral protein 16; artificial transcription factor \\
\hline GWAS & Genome wide genetic association studies \\
\hline HTP & High throughput \\
\hline i.e. & That is/id est \\
\hline IQ & Inteligence quotient \\
\hline k & Kilo \\
\hline $\mathrm{kb}$ & Kilo bases \\
\hline $\mathrm{KO}$ & Knock out \\
\hline M & Molar \\
\hline MAPK & Mitogen activated protein kinases \\
\hline MDMA & 3,4-methylenedioxy- $\mathrm{N}$-methylamphetamine \\
\hline $\min$ & Minutes \\
\hline MRI & Magnetic Resonance imaging \\
\hline $\mathrm{n}$ & Nano \\
\hline n.c. & Not calculated \\
\hline
\end{tabular}




\begin{tabular}{|l|l|}
\hline NCC & NIH clinical colection \\
\hline NIH & National Institute of Health \\
\hline NMDA & N-methyl-D-aspartate \\
\hline NRG1 & Neuregulin1 \\
\hline PCP & Phencyclidine \\
\hline PCR & Polymerase chain reaction \\
\hline PI3K & Phodphoinositide 3.kinase \\
\hline PLL & Poly-L-Lysin \\
\hline PNS & Peripheral nervous system \\
\hline PPI & Protein-protein-interaction \\
\hline PTPRZ1 & Receptor-type tyrosine-protein phosphatase zeta \\
\hline PV & Parvalbumin \\
\hline RLU & Relative luciferase units \\
\hline RTK & Receptor tyrosine kinase \\
\hline SD & Standard deviation \\
\hline sec & Seconds \\
\hline SEM & Standard error of the mean \\
\hline SHC1 & SH2 domains containing transforming protein \\
\hline SNP & Single nucleotide polymorphisms \\
\hline SRC & Sarcoma tyrosine kinase \\
\hline STAT5A & Signal transducer and activator of transcription 5a \\
\hline SV40 & Simian virus 40 \\
\hline SZ & Schizophrenia \\
\hline TACE & TNFalpha converting enzyme \\
\hline TEV & Tobacco etch virus \\
\hline THC & Tetra hydro canabinol \\
\hline TK & Thymidine kinase \\
\hline UV & Ulta violet \\
\hline V & Volt \\
\hline
\end{tabular}




\section{Curriculum vitae}

Personal data

\author{
Name \\ Place of birth \\ Nationality
}

Education

2008-currente

2008

2007-2008

2002-2007

2001-2002

2001

1998-2001

1992-1998

1988-1992

\author{
Wilko Hinrichs \\ Norderney \\ German
}

\section{Graduate student GGNB}

Prof. Nave, Department of Neurogenetics

Max-Planck-Institute of Experimental Medicine, Göttingen

\section{Diploma in biology}

Speciality in Genetics, Biochemistry, Historic Anthropology

Georg-August-University, Göttingen

Diploma student

Prof. Nave, Department of Neurogenetics

Max-Planck-Institute of Experimental Medicine, Göttingen

\section{Student GAU}

Biology

Georg-August-University Göttingen

\section{German Airforce}

Hptm. Wenninger

5.LwAusbRgt1 Budle Netherlands

\section{Abitur}

\section{Gymnasium}

Ulrichs Gymnasium Norden

Kooperative Gesamtschule

KGS Norderney

Grundschule / Primary school

Grundschule Norderney 
Professional Qualifications

2003

Research assistant

PD Dr. Friedhard Raschke

Institut für Rehabilitationsforschung

LVA Klinik Norderney

2004-2007

Research assistant

Prof. Nave, Department of Neurogenetics

Max-Planck-Institute of Experimental Medicine, Göttingen

\section{Own publications}

Velanac, V., Unterbarnscheidt, T., Hinrichs, W., Gummert, M.N., Fischer, T.M.,Möbius, W., Nave, K.-A., and Schwab, M.H., 2012, Bace1 processing of NRG1 type III produces a myelininducing signal but is not essential for the stimulation of myelination: Glia, v. 60, no. 2, p. 203-217, doi: 10.1002/glia.21255. 


\section{Apendix, List of Drugs NCC201/NCC003}

\subsubsection{NIH1-NCC201}

\begin{tabular}{|c|c|c|c|c|}
\hline NCC_SAMPLE & PI. & Well & Supplier & NCC_STRUCTURE_SYNONYMS[2] \\
\hline SAM002264608 & 1 & $\mathrm{~A} 02$ & LightBiologicals & DOXEPIN HYDROCHLORIDE \\
\hline SAM002264609 & 1 & B02 & LightBiologicals & DIPYRIDAMOLE \\
\hline SAM002264610 & 1 & $\mathrm{C} 02$ & LightBiologicals & Propofol \\
\hline SAM002264611 & 1 & D02 & LightBiologicals & ETHACRYNIC ACID \\
\hline SAM002264612 & 1 & E02 & LightBiologicals & FLUTAMIDE \\
\hline SAM002264613 & 1 & $\mathrm{~F} 02$ & LightBiologicals & FENOFIBRATE \\
\hline SAM002264614 & 1 & G02 & LightBiologicals & FUROSEMIDE \\
\hline SAM002264615 & 1 & $\mathrm{H} 02$ & LightBiologicals & 5-FLUOROURACIL \\
\hline SAM002264616 & 1 & $\mathrm{~A} 03$ & LightBiologicals & Folic acid \\
\hline SAM002264617 & 1 & B03 & LightBiologicals & HYDROCORTISONE \\
\hline SAM002264618 & 1 & $\mathrm{CO3}$ & LightBiologicals & Cortell \\
\hline SAM002264619 & 1 & D03 & LightBiologicals & IBUPROFEN \\
\hline SAM002264620 & 1 & E03 & LightBiologicals & KETOPROFEN \\
\hline SAM002264621 & 1 & F03 & LightBiologicals & MINOCYCLINE HYDROCHLORIDE \\
\hline SAM002264623 & 1 & G03 & LightBiologicals & MICONAZOLE NITRATE \\
\hline SAM002297829 & 1 & $\mathrm{H} 03$ & LightBiologicals & METYRAPONE \\
\hline SAM002264625 & 1 & $\mathrm{~A} 04$ & LightBiologicals & NORFLOXACIN \\
\hline SAM002264627 & 1 & B04 & LightBiologicals & NADOLOL \\
\hline SAM002264628 & 1 & $\mathrm{C04}$ & LightBiologicals & Disipal \\
\hline SAM002264629 & 1 & D04 & LightBiologicals & OFLOXACIN \\
\hline SAM002264631 & 1 & E04 & LightBiologicals & PINDOLOL \\
\hline SAM002264632 & 1 & F04 & LightBiologicals & PRAZIQUANTEL \\
\hline SAM002264634 & 1 & G04 & LightBiologicals & Benzenebutanoic Acid \\
\hline SAM002264635 & 1 & $\mathrm{H} 04$ & LightBiologicals & PREDNISOLONE ACETATE \\
\hline SAM002264636 & 1 & A05 & LightBiologicals & Phenergan \\
\hline SAM002264637 & 1 & B05 & LightBiologicals & PERPHENAZINE \\
\hline SAM002264639 & 1 & $\mathrm{C} 05$ & LightBiologicals & Prednisolone \\
\hline SAM002264640 & 1 & D05 & LightBiologicals & PRILOCAINE HYDROCHLORIDE \\
\hline SAM002264641 & 1 & E05 & LightBiologicals & Prednisone \\
\hline SAM002264642 & 1 & F05 & LightBiologicals & DL-PENICILLAMINE \\
\hline SAM002264643 & 1 & G05 & LightBiologicals & PIPERACILLIN SODIUM SALT \\
\hline SAM002264644 & 1 & $\mathrm{H} 05$ & LightBiologicals & Quinidine hydrochloride monohydrate \\
\hline SAM002264645 & 1 & $\mathrm{~A} 06$ & LightBiologicals & Ranitidine hydrochloride \\
\hline SAM002264646 & 1 & B06 & LightBiologicals & RIFAMPICIN \\
\hline SAM002264647 & 1 & $\mathrm{C} 06$ & LightBiologicals & Retinoic acid \\
\hline SAM002264648 & 1 & D06 & LightBiologicals & Spironolactone \\
\hline SAM002264649 & 1 & E06 & LightBiologicals & Trimethoprim \\
\hline SAM002264650 & 1 & F06 & LightBiologicals & Tyzine \\
\hline SAM002264651 & 1 & G06 & LightBiologicals & L-THYROXINE \\
\hline SAM002264652 & 1 & $\mathrm{H} 06$ & LightBiologicals & Artane \\
\hline SAM002264653 & 1 & $\mathrm{~A} 07$ & LightBiologicals & URSODEOXYCHOLIC ACID \\
\hline SAM002554879 & 1 & B07 & LightBiologicals & Dapsone \\
\hline SAM002554881 & 1 & $\mathrm{C} 07$ & LightBiologicals & Symmetrel \\
\hline SAM002554882 & 1 & D07 & LightBiologicals & WARFARIN SODIUM \\
\hline SAM002554883 & 1 & E07 & LightBiologicals & Acetazolamide \\
\hline
\end{tabular}




\begin{tabular}{|c|c|c|c|c|}
\hline SAM002554884 & 1 & F07 & LightBiologicals & Allopurinol \\
\hline SAM002554885 & 1 & G07 & LightBiologicals & ATROPINE \\
\hline SAM002554914 & 1 & $\mathrm{H} 07$ & LightBiologicals & Nalidixic Acid \\
\hline "SAM003107539 & 1 & A08 & LightBiologicals & "'"3 \\
\hline SAM002703134 & 1 & B08 & LightBiologicals & HYDROFLUMETHIAZIDE \\
\hline SAM002554886 & 1 & $\mathrm{C} 08$ & LightBiologicals & Annoyltin \\
\hline SAM002554887 & 1 & D08 & LightBiologicals & Busulfan \\
\hline SAM002554888 & 1 & E08 & LightBiologicals & Chlorzoxazone \\
\hline SAM002554891 & 1 & F08 & LightBiologicals & Chlorothiazide \\
\hline SAM002554892 & 1 & G08 & LightBiologicals & Cimetidine \\
\hline SAM002554889 & 1 & $\mathrm{H} 08$ & LightBiologicals & Carisoprodol \\
\hline SAM002554890 & 1 & A09 & LightBiologicals & Chlorpropamide \\
\hline "SAM002554894 & 1 & B09 & LightBiologicals & Bentyl \\
\hline SAM002554895 & 1 & $\mathrm{C} 09$ & LightBiologicals & Chloroxine \\
\hline SAM002554896 & 1 & D09 & LightBiologicals & Diflunisal \\
\hline SAM002554898 & 1 & E09 & LightBiologicals & Econazole Nitrate \\
\hline SAM002554899 & 1 & F09 & LightBiologicals & Ethionamide \\
\hline SAM002554900 & 1 & G09 & LightBiologicals & Methocarbamol \\
\hline SAM002554901 & 1 & $\mathrm{HO9}$ & LightBiologicals & Hydrochlorothiazide \\
\hline SAM002554902 & 1 & $\mathrm{~A} 10$ & LightBiologicals & Vistaril Pamoate \\
\hline SAM002554903 & 1 & $\mathrm{~B} 10$ & LightBiologicals & Hexachlorophene \\
\hline SAM002554904 & 1 & $\mathrm{C} 10$ & LightBiologicals & Isoniazid \\
\hline SAM002554905 & 1 & D10 & LightBiologicals & Duvadilan \\
\hline SAM002554906 & 1 & E10 & LightBiologicals & Isuprel \\
\hline SAM002554907 & 1 & F10 & LightBiologicals & Triclosan \\
\hline SAM002554908 & 1 & G10 & LightBiologicals & Mefenamic Acid \\
\hline "SAM002554910 & 1 & $\mathrm{H} 10$ & LightBiologicals & Cantil \\
\hline SAM002554911 & 1 & $\mathrm{~A} 11$ & LightBiologicals & Maxolon \\
\hline SAM002554912 & 1 & B11 & LightBiologicals & Methyldopa \\
\hline SAM002554913 & 1 & $\mathrm{C} 11$ & LightBiologicals & NITROFURANTOIN \\
\hline SAM002554915 & 1 & D11 & LightBiologicals & Pamelor \\
\hline SAM002554916 & 1 & E11 & LightBiologicals & Albalon \\
\hline SAM002554917 & 1 & $\mathrm{~F} 11$ & LightBiologicals & Nicotinic Acid \\
\hline SAM002554918 & 1 & G11 & LightBiologicals & Norflex \\
\hline SAM002554919 & 1 & $\mathrm{H} 11$ & LightBiologicals & Oxytetracycline hydrochloride \\
\hline SAM002554920 & 2 & $\mathrm{~A} 02$ & LightBiologicals & Novocain \\
\hline SAM002554921 & 2 & B02 & LightBiologicals & Pyrimethamine \\
\hline SAM002554922 & 2 & $\mathrm{C} 02$ & LightBiologicals & Pro-Banthine \\
\hline SAM002554923 & 2 & D02 & LightBiologicals & Probenecid \\
\hline SAM002554924 & 2 & E02 & LightBiologicals & PYRIDINE-2-ALDOXIME METHOCHLORIDE \\
\hline SAM002554925 & 2 & F02 & LightBiologicals & Primidone \\
\hline SAM002554926 & 2 & G02 & LightBiologicals & Propylthiouracil \\
\hline SAM002554927 & 2 & $\mathrm{H} 02$ & LightBiologicals & Pyrazinamide \\
\hline SAM002554928 & 2 & $\mathrm{~A} 03$ & LightBiologicals & Pronestyl \\
\hline SAM002554929 & 2 & B03 & LightBiologicals & Sulfisoxazole \\
\hline SAM002554930 & 2 & $\mathrm{CO3}$ & LightBiologicals & Sulfamethoxazole \\
\hline SAM002554931 & 2 & D03 & LightBiologicals & Sulfacetamide \\
\hline SAM002554932 & 2 & E03 & LightBiologicals & Sulfinpyrazone \\
\hline SAM002554933 & 2 & F03 & LightBiologicals & Sulindac \\
\hline SAM002554934 & 2 & G03 & LightBiologicals & TETRACYCLINE \\
\hline SAM002554935 & 2 & $\mathrm{H} 03$ & LightBiologicals & Theophylline \\
\hline SAM002554936 & 2 & $\mathrm{~A} 04$ & LightBiologicals & Tolbutamide \\
\hline
\end{tabular}




\begin{tabular}{|c|c|c|c|c|}
\hline "SAM002554937 & 2 & B04 & LightBiologicals & Triamterene \\
\hline SAM002554938 & 2 & C04 & LightBiologicals & Intropin \\
\hline SAM002564189 & 2 & D04 & LightBiologicals & AMOXAPINE \\
\hline SAM002564191 & 2 & E04 & LightBiologicals & Adenine 9-beta \\
\hline SAM002564193 & 2 & F04 & LightBiologicals & ATENOLOL \\
\hline SAM002703133 & 2 & G04 & LightBiologicals & Tamoxifen \\
\hline SAM002564195 & 2 & $\mathrm{H} 04$ & LightBiologicals & BUMETANIDE \\
\hline SAM002564250 & 2 & A05 & LightBiologicals & CLOBETASOL PROPIONATE \\
\hline SAM002564196 & 2 & B05 & LightBiologicals & Sonazine \\
\hline SAM002564200 & 2 & $\mathrm{C} 05$ & LightBiologicals & CEFAZOLIN SODIUM SALT \\
\hline SAM002564201 & 2 & D05 & LightBiologicals & CAPTOPRIL \\
\hline SAM002564202 & 2 & E05 & LightBiologicals & CHLORAMBUCIL \\
\hline SAM002564251 & 2 & F05 & LightBiologicals & CEFOXITIN SODIUM SALT \\
\hline SAM002564203 & 2 & G05 & LightBiologicals & DANAZOL \\
\hline SAM002564204 & 2 & $\mathrm{H} 05$ & LightBiologicals & (+)-CIS-DILTIAZEM HYDROCHLORIDE \\
\hline SAM002564205 & 2 & A06 & LightBiologicals & DIGOXIN \\
\hline SAM002564206 & 2 & B06 & LightBiologicals & 17-BETA-ESTRADIOL 17-VALERATE \\
\hline SAM002564207 & 2 & $\mathrm{C} 06$ & LightBiologicals & EDROPHONIUM CHLORIDE \\
\hline SAM002564208 & 2 & D06 & LightBiologicals & FLUOCINOLONE ACETONIDE \\
\hline SAM002564209 & 2 & E06 & LightBiologicals & Flurbiprofen \\
\hline SAM002564210 & 2 & F06 & LightBiologicals & GLIPIZIDE \\
\hline SAM002564211 & 2 & G06 & LightBiologicals & GEMFIBROZIL \\
\hline SAM002564212 & 2 & H06 & LightBiologicals & Glyburide \\
\hline SAM002564213 & 2 & $\mathrm{~A} 07$ & LightBiologicals & HYDROCORTISONE HEMISUCCINATE \\
\hline SAM002564214 & 2 & B07 & LightBiologicals & INDAPAMIDE \\
\hline SAM002564215 & 2 & $\mathrm{C} 07$ & LightBiologicals & IPRATROPIUM BROMIDE MONOHYDRATE \\
\hline SAM002564216 & 2 & D07 & LightBiologicals & Tofranil \\
\hline SAM002564217 & 2 & E07 & LightBiologicals & LABETALOL HYDROCHLORIDE \\
\hline SAM002564218 & 2 & F07 & LightBiologicals & Imodium \\
\hline SAM002564219 & 2 & G07 & LightBiologicals & Pro-Amatine \\
\hline SAM002564220 & 2 & H07 & LightBiologicals & Medroxyprogesterone 17-acetate \\
\hline SAM002564222 & 2 & $\mathrm{~A} 08$ & LightBiologicals & 19-NORETHINDRONE ACETATE \\
\hline SAM002564223 & 2 & B08 & LightBiologicals & 19-Norethindrone \\
\hline SAM002564224 & 2 & $\mathrm{C} 08$ & LightBiologicals & NICOTINE \\
\hline SAM002564254 & 2 & D08 & LightBiologicals & Cardene \\
\hline SAM002564225 & 2 & E08 & LightBiologicals & NABUMETONE \\
\hline SAM002564226 & 2 & F08 & LightBiologicals & OXYBUTYNIN CHLORIDE \\
\hline SAM002564227 & 2 & G08 & LightBiologicals & Mestinon \\
\hline SAM002564228 & 2 & $\mathrm{H} 08$ & LightBiologicals & Rythmol \\
\hline SAM002564229 & 2 & A09 & LightBiologicals & Pfizerpen \\
\hline SAM002564230 & 2 & B09 & LightBiologicals & Valproic Acid \\
\hline SAM002564231 & 2 & $\mathrm{Co9}$ & LightBiologicals & Kemadrin \\
\hline "SAM002564232 & 2 & D09 & LightBiologicals & Proxymetacaine \\
\hline SAM002703137 & 2 & E09 & LightBiologicals & NALOXONE HYDROCHLORIDE \\
\hline SAM002564233 & 2 & F09 & LightBiologicals & SPECTINOMYCIN DIHYDROCHLORIDE PENTAHYDRATE \\
\hline SAM002564235 & 2 & G09 & LightBiologicals & TROPICAMIDE \\
\hline SAM002564236 & 2 & $\mathrm{H} 09$ & LightBiologicals & TOLAZAMIDE \\
\hline SAM002564237 & 2 & A10 & LightBiologicals & TRIAMCINOLONE ACETONIDE \\
\hline SAM002564238 & 2 & $\mathrm{~B} 10$ & LightBiologicals & $\mathrm{S}(-)-$ Timolol maleate \\
\hline SAM002564239 & 2 & C10 & LightBiologicals & THIABENDAZOLE \\
\hline SAM002564240 & 2 & D10 & LightBiologicals & THIORIDAZINE HYDROCHLORIDE \\
\hline SAM002564241 & 2 & E10 & LightBiologicals & Altretamine \\
\hline
\end{tabular}


Apendix

\begin{tabular}{|c|c|c|c|c|}
\hline SAM002564257 & 2 & F10 & LightBiologicals & Phylloquinone \\
\hline SAM002564242 & 2 & G10 & LightBiologicals & Eryped \\
\hline SAM002564244 & 2 & $\mathrm{H} 10$ & LightBiologicals & Dibenzyline \\
\hline "SAM002564258 & 2 & A11 & LightBiologicals & 6ALPHA-METHYL-11BETA-HYDROXYPROGESTERONE \\
\hline SAM002564245 & 2 & B11 & LightBiologicals & Thalidomide \\
\hline SAM002589919 & 2 & C11 & LightBiologicals & Aminolevulinic Acid \\
\hline SAM002589920 & 2 & D11 & LightBiologicals & Carbinoxamine Maleate \\
\hline SAM002589921 & 2 & E11 & LightBiologicals & Demeclocycline \\
\hline SAM002589925 & 2 & F11 & LightBiologicals & Westcort \\
\hline SAM002589926 & 2 & G11 & LightBiologicals & DEPRENALIN \\
\hline SAM002589927 & 2 & $\mathrm{H} 11$ & LightBiologicals & 6-[2-ETHOXY-1-NAPHTHAMIDO]-PENICILLIN SODIUM SALT \\
\hline SAM002589929 & 3 & A02 & LightBiologicals & Primaquine Diphosphate \\
\hline SAM002589930 & 3 & B02 & LightBiologicals & Micropenin \\
\hline SAM002589932 & 3 & $\mathrm{C} 02$ & LightBiologicals & DOXYCYCLINE \\
\hline "SAM002699895 & 3 & D02 & LightBiologicals & Beclomethasone dipropionate \\
\hline SAM002589934 & 3 & E02 & LightBiologicals & Cromolyn Sodium \\
\hline SAM002589935 & 3 & F02 & LightBiologicals & Priscoline \\
\hline SAM002589937 & 3 & G02 & LightBiologicals & Mercaptopurine \\
\hline SAM002589938 & 3 & $\mathrm{H} 02$ & LightBiologicals & Azathioprine \\
\hline SAM002589939 & 3 & $\mathrm{~A} 03$ & LightBiologicals & Albendazole \\
\hline SAM002589940 & 3 & B03 & LightBiologicals & Griseofulvin \\
\hline SAM002589936 & 3 & $\mathrm{C} 03$ & LightBiologicals & Lincomycin hydrochloride \\
\hline SAM002589943 & 3 & D03 & LightBiologicals & Methazolamide \\
\hline SAM002589944 & 3 & E03 & LightBiologicals & Terbutaline Sulfate \\
\hline SAM002589979 & 3 & F03 & LightBiologicals & Mupirocin \\
\hline SAM002589945 & 3 & G03 & LightBiologicals & FLUOCINOLONE ACETONIDE 21-ACETATE \\
\hline SAM002699894 & 3 & $\mathrm{H} 03$ & LightBiologicals & Mefloquine hydrochloride \\
\hline SAM002589947 & 3 & A04 & LightBiologicals & Floxuridine \\
\hline SAM002699896 & 3 & B04 & LightBiologicals & MITOXANTRONE \\
\hline SAM002699897 & 3 & $\mathrm{C} 04$ & LightBiologicals & ENALAPRIL MALEATE \\
\hline SAM002699898 & 3 & D04 & LightBiologicals & BUDESONIDE \\
\hline SAM002699899 & 3 & E04 & LightBiologicals & RAMIPRIL \\
\hline SAM002699893 & 3 & F04 & LightBiologicals & DEPO-MEDROL \\
\hline SAM002699901 & 3 & G04 & LightBiologicals & (+/-)-NOREPINEPHRINE HYDROCHLORIDE \\
\hline SAM002699903 & 3 & $\mathrm{H} 04$ & LightBiologicals & AMCINONIDE \\
\hline SAM002699904 & 3 & A05 & LightBiologicals & Clomid \\
\hline SAM003107541 & 3 & B05 & LightBiologicals & PHENTOLAMINE HCL \\
\hline SAM002548956 & 3 & $\mathrm{C} 05$ & Enamine & FLUDARABINE \\
\hline SAM002548950 & 3 & D05 & Enamine & Testosterone \\
\hline SAM002548955 & 3 & E05 & Enamine & Isotretinoin \\
\hline SAM002548951 & 3 & F05 & Enamine & Methimazole \\
\hline SAM002548957 & 3 & G05 & Enamine & Zonisamide \\
\hline SAM002548958 & 3 & $\mathrm{H} 05$ & Enamine & Brimonidine \\
\hline SAM002548959 & 3 & A06 & Enamine & Mebendazole \\
\hline SAM002548969 & 3 & B06 & Enamine & Duremesin \\
\hline SAM002548968 & 3 & $\mathrm{C} 06$ & Enamine & Flecainide Acetate \\
\hline SAM002548966 & 3 & D06 & Enamine & Dilantin \\
\hline SAM002548945 & 3 & E06 & Enamine & Miochol \\
\hline SAM002703135 & 3 & F06 & LightBiologicals & Dantrolene sodium salt \\
\hline SAM002548948 & 3 & G06 & Enamine & Dexamethasone \\
\hline SAM002548938 & 3 & H06 & Enamine & Cogentin Mesylate \\
\hline SAM002548936 & 3 & A07 & Enamine & Ganciclovir \\
\hline
\end{tabular}


Apendix

\begin{tabular}{|c|c|c|c|c|}
\hline SAM002548937 & 3 & B07 & Enamine & Mesna \\
\hline SAM002548942 & 3 & $\mathrm{C} 07$ & Enamine & Meclomen \\
\hline SAM002589905 & 3 & D07 & Enamine & Fluconazole \\
\hline SAM002548965 & 3 & E07 & Enamine & Metaproterenol \\
\hline SAM002548974 & 3 & F07 & Enamine & Methoxsalen \\
\hline SAM002548963 & 3 & G07 & Enamine & Chloramphenicol \\
\hline SAM002548961 & 3 & $\mathrm{H} 07$ & Enamine & Tizanidine hydrochloride \\
\hline SAM002548935 & 3 & $\mathrm{~A} 08$ & Enamine & Paroxetine \\
\hline SAM002548934 & 3 & B08 & Enamine & mirtazapine \\
\hline SAM002548930 & 3 & $\mathrm{C} 08$ & Enamine & Etomidate \\
\hline SAM002548933 & 3 & D08 & Enamine & Moban \\
\hline SAM002548940 & 3 & E08 & Enamine & fluvastatin \\
\hline SAM002548931 & 3 & F08 & Enamine & Urecholine \\
\hline SAM002589901 & 3 & G08 & Enamine & Cefuroxime \\
\hline SAM002548983 & 3 & $\mathrm{H} 08$ & Enamine & Cytoxan \\
\hline SAM002548982 & 3 & A09 & Enamine & Eszopiclone \\
\hline SAM002264598 & 3 & B09 & LightBiologicals & Bendrofluazide \\
\hline SAM002548975 & 3 & $\mathrm{Cog}$ & Enamine & Evista \\
\hline SAM002548971 & 3 & D09 & Enamine & zidovudine \\
\hline SAM002548976 & 3 & E09 & Enamine & Clozapine \\
\hline SAM002264590 & 3 & F09 & LightBiologicals & Ampicillin Sodium \\
\hline SAM002264591 & 3 & G09 & LightBiologicals & ACEBUTOLOL HYDROCHLORIDE \\
\hline SAM002264592 & 3 & $\mathrm{H} 09$ & LightBiologicals & AMOXICILLIN CRYSTALLINE \\
\hline SAM002699891 & 3 & $\mathrm{~A} 10$ & LightBiologicals & (+/-)-Epinephrine hydrochloride \\
\hline SAM002264595 & 3 & B10 & LightBiologicals & 5-Azacytidine \\
\hline SAM002264597 & 3 & $\mathrm{C} 10$ & LightBiologicals & Buspar \\
\hline SAM002264596 & 3 & D10 & LightBiologicals & Flumadine \\
\hline SAM002548978 & 3 & E10 & Enamine & Podofilox \\
\hline SAM002264599 & 3 & F10 & LightBiologicals & D-CYCLOSERINE \\
\hline SAM002264600 & 3 & G10 & LightBiologicals & CORTISONE ACETATE \\
\hline SAM002264601 & 3 & $\mathrm{H} 10$ & LightBiologicals & Anafranil \\
\hline SAM002264603 & 3 & A11 & LightBiologicals & Carbamazepine \\
\hline SAM002699890 & 3 & $\mathrm{~B} 11$ & LightBiologicals & Memantine hydrochloride \\
\hline SAM002264605 & 3 & $\mathrm{C} 11$ & LightBiologicals & Norpramin \\
\hline SAM002264606 & 3 & D11 & LightBiologicals & Mexitil \\
\hline SAM002264607 & 3 & E11 & LightBiologicals & Norpace \\
\hline SAM002589948 & 3 & $\mathrm{~F} 11$ & LightBiologicals & STAVUDINE \\
\hline SAM002589981 & 3 & G11 & LightBiologicals & Doxazosin \\
\hline SAM002589949 & 3 & $\mathrm{H} 11$ & LightBiologicals & Minoxidil \\
\hline SAM002699889 & 4 & $\mathrm{~A} 02$ & LightBiologicals & Inderal \\
\hline SAM002589951 & 4 & B02 & LightBiologicals & Ribavirin \\
\hline SAM002589982 & 4 & $\mathrm{C} 02$ & LightBiologicals & Terazosin \\
\hline SAM002589983 & 4 & D02 & LightBiologicals & Chlorthalidone \\
\hline SAM002589984 & 4 & E02 & LightBiologicals & METHYLPREDNISOLONE \\
\hline SAM002589985 & 4 & $\mathrm{~F} 02$ & LightBiologicals & Phenelzine \\
\hline SAM002589986 & 4 & G02 & LightBiologicals & NALTREXONE HYDROCHLORIDE \\
\hline SAM002589987 & 4 & $\mathrm{H} 02$ & LightBiologicals & Glycopyrrolate \\
\hline SAM002589988 & 4 & $\mathrm{~A} 03$ & LightBiologicals & Ethambutol \\
\hline SAM002589989 & 4 & B03 & LightBiologicals & Cetirizine \\
\hline SAM002589990 & 4 & $\mathrm{C03}$ & LightBiologicals & DICLOXACILLIN SODIUM \\
\hline SAM002589991 & 4 & D03 & LightBiologicals & Meloxicam \\
\hline SAM002589992 & 4 & E03 & LightBiologicals & DAUNORUBICIN HYDROCHLORIDE \\
\hline
\end{tabular}




\begin{tabular}{|c|c|c|c|c|}
\hline SAM002589953 & 4 & F03 & LightBiologicals & RIFAPENTINE \\
\hline SAM002589954 & 4 & G03 & LightBiologicals & Penicillin V \\
\hline SAM002589955 & 4 & $\mathrm{H} 03$ & LightBiologicals & Gatifloxacin \\
\hline SAM002589956 & 4 & $\mathrm{~A} 04$ & LightBiologicals & clopidogrel \\
\hline SAM002643511 & 4 & B04 & LightBiologicals & CEFOTAXIME SODIUM SALT \\
\hline SAM002589994 & 4 & $\mathrm{C04}$ & LightBiologicals & LAMIVUDINE \\
\hline SAM002589958 & 4 & D04 & LightBiologicals & Ondansetron \\
\hline SAM002589959 & 4 & E04 & LightBiologicals & Betamethasone \\
\hline SAM002589995 & 4 & F04 & LightBiologicals & Celecoxib \\
\hline SAM002589996 & 4 & G04 & LightBiologicals & 4-(AMINOMETHYL)BENZENESULFONAMIDE ACETATE \\
\hline SAM002589997 & 4 & $\mathrm{H} 04$ & LightBiologicals & THIOTHIXENE \\
\hline SAM002589960 & 4 & A05 & LightBiologicals & Citalopram \\
\hline SAM002589961 & 4 & B05 & LightBiologicals & Azithromycin \\
\hline SAM002589963 & 4 & $\mathrm{C} 05$ & LightBiologicals & Lovastatin \\
\hline SAM002589964 & 4 & D05 & LightBiologicals & Aminoglutethimide \\
\hline SAM002589965 & 4 & E05 & LightBiologicals & Prozac \\
\hline SAM002589966 & 4 & F05 & LightBiologicals & FluniSOLIDe \\
\hline SAM002589967 & 4 & G05 & LightBiologicals & Acyclovir \\
\hline SAM002589968 & 4 & $\mathrm{H} 05$ & LightBiologicals & Etodolac \\
\hline SAM002589969 & 4 & A06 & LightBiologicals & Simvastatin \\
\hline SAM002589970 & 4 & B06 & LightBiologicals & Rifabutin \\
\hline SAM002589971 & 4 & $\mathrm{C} 06$ & LightBiologicals & Felodipine \\
\hline SAM002589972 & 4 & D06 & LightBiologicals & Quinapril hydrochloride \\
\hline SAM002589973 & 4 & E06 & LightBiologicals & Acitretin \\
\hline SAM002700173 & 4 & F06 & LightBiologicals & Allegra \\
\hline SAM002700174 & 4 & G06 & LightBiologicals & Fluorometholone \\
\hline SAM002700175 & 4 & $\mathrm{H} 06$ & LightBiologicals & Sertraline \\
\hline SAM002703129 & 4 & $\mathrm{~A} 07$ & LightBiologicals & CARBIDOPA \\
\hline
\end{tabular}

Table 9. NCC201

\subsubsection{NIH2-NCCO03}

\begin{tabular}{|l|r|l|l|l|}
\hline NCC_SAMPLE & PI. & Well & Supplier & NCC_STRUCTURE_SYNONYMS[2] \\
\hline SAM001247063 & 1 & A02 & Tocris Bioscience & Nalbuphine \\
\hline SAM001247072 & 1 & B02 & Tocris Bioscience & Raclopride \\
\hline SAM001247069 & 1 & C02 & Tocris Bioscience & Zacopride \\
\hline SAM001247068 & 1 & D02 & Tocris Bioscience & SKF 83566 \\
\hline SAM001246964 & 1 & E02 & Tocris Bioscience & 3'-deoxydenosine \\
\hline SAM001246965 & 1 & F02 & Tocris Bioscience & AM 404 \\
\hline SAM001246962 & 1 & G02 & Tocris Bioscience & PILOCARPINE HYDROCHLORIDE \\
\hline SAM001246963 & 1 & H02 & Tocris Bioscience & NIFEDIPINE \\
\hline SAM001246961 & 1 & A03 & Tocris Bioscience & Flurbiprofen \\
\hline SAM001247015 & 1 & B03 & Tocris Bioscience & 3-HYDROXY-1,2-DIMETHYL-4(1H)-PYRIDONE \\
\hline SAM001247061 & 1 & C03 & Tocris Bioscience & \\
\hline SAM001247062 & 1 & D03 & Tocris Bioscience & d-3-Methoxy-N-methylmorphinan hydrobromide \\
\hline SAM001247059 & 1 & E03 & Tocris Bioscience & Duloxetine \\
\hline SAM001247060 & 1 & F03 & Tocris Bioscience & $\begin{array}{l}\text { Glycine, N-[2-[(acetylthio)methyl]-1-oxo-3-phenylpropyl]- } \\
\text { phenylmethyl ester [CAS] }\end{array}$ \\
\hline SAM001247057 & 1 & G03 & Tocris Bioscience & $\begin{array}{l}\text { Benzeneacetic acid, } \\
\text { monosodium salt [CAS] }\end{array}$ \\
\hline SAM001247039 & 1 & H03 & Tocris Bioscience & PROGESTERophenyl)amino]-, \\
\hline SAM001247033 & 1 & A04 & Tocris Bioscience & FAMOTIDINE \\
\hline SAM001246999 & 1 & B04 & Tocris Bioscience & SR 57,227A \\
\hline SAM001247003 & 1 & C04 & Tocris Bioscience & Pancuronium \\
\hline
\end{tabular}




\begin{tabular}{|c|c|c|c|c|}
\hline SAM001247010 & 1 & D04 & Tocris Bioscience & METRONIDAZOLE \\
\hline SAM001246967 & 1 & E04 & Tocris Bioscience & 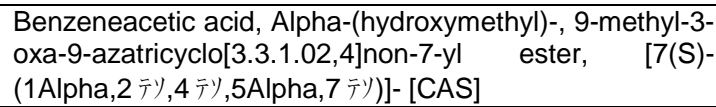 \\
\hline SAM001246968 & 1 & $\mathrm{~F} 04$ & Tocris Bioscience & $\begin{array}{l}\text { Benzeneacetonitrile, Alpha-[3-[[2-(3,4- } \\
\text { dimethoxyphenyl)ethyl]methylamino]propyl]-3,4- } \\
\text { dimethoxy-Alpha-(1-methylethyl)-, (R)-[CAS] }\end{array}$ \\
\hline SAM001246969 & 1 & G04 & Tocris Bioscience & DEPRENALIN \\
\hline SAM001246970 & 1 & $\mathrm{H} 04$ & Tocris Bioscience & Capsaicin \\
\hline SAM001246971 & 1 & A05 & Tocris Bioscience & SALBUTAMOL SULFATE \\
\hline SAM001246972 & 1 & B05 & Tocris Bioscience & (ア)-Vesamicol hydrochloride \\
\hline SAM001246993 & 1 & $\mathrm{C} 05$ & Tocris Bioscience & Picrotin - Picrotoxinin \\
\hline SAM001247000 & 1 & D05 & Tocris Bioscience & Terazosin \\
\hline SAM001247027 & 1 & E05 & Tocris Bioscience & diphenylcyclopropenone \\
\hline SAM001247016 & 1 & $\mathrm{~F} 05$ & Tocris Bioscience & 4-Thiazolidinecarboxylic acid, 2-oxo-, (R)- [CAS] \\
\hline SAM001247051 & 1 & G05 & Tocris Bioscience & Mesoridazine \\
\hline SAM001247053 & 1 & $\mathrm{H} 05$ & Tocris Bioscience & $\begin{array}{l}3(2 \mathrm{H}) \text {-Pyridazinone, } \\
\text { methoxyphenyl]- [CAS] }\end{array}$ \\
\hline SAM001247054 & 1 & A06 & Tocris Bioscience & $\begin{array}{l}\text { 10H-Phenothiazine, } \\
\text { piperazinyl)propyl- }[\mathrm{CAS}]\end{array}$ 2-chloro-10-[3-(4-methyl-1- \\
\hline SAM001247055 & 1 & B06 & Tocris Bioscience & $\begin{array}{l}1 \mathrm{H} \text {-Cyclopenta[b]quinolin-9-amine, } \\
\text { hexahydro-, monohydrochloride- }[\mathrm{CAS}]\end{array}$ \\
\hline SAM001247056 & 1 & $\mathrm{C} 06$ & Tocris Bioscience & CLOTRIMAZOLE \\
\hline SAM001246987 & 1 & $\mathrm{D} 06$ & Tocris Bioscience & LORATADINE \\
\hline SAM001247037 & 1 & E06 & Tocris Bioscience & PHENELZINE SULFATE SALT \\
\hline SAM001246997 & 1 & F06 & Tocris Bioscience & Riluzole \\
\hline SAM001247004 & 1 & G06 & Tocris Bioscience & Naltrindole \\
\hline SAM001247026 & 1 & $\mathrm{H} 06$ & Tocris Bioscience & Nornicotine \\
\hline SAM001247052 & 1 & $\mathrm{~A} 07$ & Tocris Bioscience & Bifemelane \\
\hline SAM001246973 & 1 & B07 & Tocris Bioscience & CGS 15943 \\
\hline SAM001246974 & 1 & $\mathrm{C} 07$ & Tocris Bioscience & Cinanserin \\
\hline SAM001246975 & 1 & $\mathrm{D} 07$ & Tocris Bioscience & Cisapride \\
\hline SAM001246981 & 1 & E07 & Tocris Bioscience & Indatraline \\
\hline SAM001247045 & 1 & $\mathrm{~F} 07$ & Tocris Bioscience & TRAZODONE HYDROCHLORIDE \\
\hline SAM001246995 & 1 & G07 & Tocris Bioscience & Prazosin \\
\hline SAM001247001 & 1 & $\mathrm{H} 07$ & Tocris Bioscience & URAPIDIL HYDROCHLORIDE \\
\hline SAM001247007 & 1 & A08 & Tocris Bioscience & $(-)$-Cotinine \\
\hline SAM001247014 & 1 & B08 & Tocris Bioscience & D-CYCLOSERINE \\
\hline SAM001246977 & 1 & $\mathrm{C} 08$ & Tocris Bioscience & Fluvoxamine \\
\hline SAM001246976 & 1 & D08 & Tocris Bioscience & Doxepin \\
\hline SAM001247046 & 1 & E08 & Tocris Bioscience & \\
\hline SAM001247023 & 1 & F08 & Tocris Bioscience & $\begin{array}{l}\text { (+)-3-HYDROXY-N-METHYLMORPHINAN } \\
\text { TARTRATE }\end{array}$ \\
\hline SAM001247049 & 1 & G08 & Tocris Bioscience & L-Ornithine, N5-[imino(methylamino)methyl]-[CAS] \\
\hline SAM001246989 & 1 & $\mathrm{H} 08$ & Tocris Bioscience & Maprotilline $\mathrm{HCl}$ \\
\hline SAM001247038 & 1 & A09 & Tocris Bioscience & Pizotyline \\
\hline SAM001247032 & 1 & B09 & Tocris Bioscience & BETA-ESTRADIOL \\
\hline SAM001247019 & 1 & $\mathrm{Co9}$ & Tocris Bioscience & N,N'-DIACETYL-1,6-DIAMINOHEXANE \\
\hline SAM001247024 & 1 & D09 & Tocris Bioscience & Diphenhydramine hydrochloride \\
\hline SAM001246978 & 1 & E09 & Tocris Bioscience & Galanthamine \\
\hline SAM001246980 & 1 & F09 & Tocris Bioscience & Ifenprodil \\
\hline SAM001247028 & 1 & G09 & Tocris Bioscience & TETRAETHYLTHIURAM DISULFIDE \\
\hline SAM001246994 & 1 & $\mathrm{H} 09$ & Tocris Bioscience & Piribedil \\
\hline SAM001246983 & 1 & $\mathrm{~A} 10$ & Tocris Bioscience & \\
\hline SAM001247047 & 1 & B10 & Tocris Bioscience & TRIPELENNAMINE HYDROCHLORIDE \\
\hline SAM001246996 & 1 & $\mathrm{C} 10$ & Tocris Bioscience & $\begin{array}{l}\text { Pyrazinecarboxamide, } \\
\text { (aminoiminomethyl)-6-chloro- [CAS] }\end{array}$ \\
\hline
\end{tabular}


Apendix

\begin{tabular}{|c|c|c|c|c|}
\hline SAM001247002 & 1 & D10 & Tocris Bioscience & $\begin{array}{l}\text { 9-AMINO-1,2,3,4-TETRAHYDROACRIDINE } \\
\text { HYDROCHLORIDE }\end{array}$ \\
\hline SAM001247008 & 1 & E10 & Tocris Bioscience & ETHYNYLESTRADIOL \\
\hline SAM001247012 & 1 & F10 & Tocris Bioscience & $\begin{array}{l}\text { 2(1H)-Pyrimidinone, 4-amino-1-テリ-D-arabinofuranosyl- } \\
\text { [CAS] }\end{array}$ \\
\hline SAM001246985 & 1 & G10 & Tocris Bioscience & $\begin{array}{lcc}\text { L-Glutamic } & \text { acid, } & \mathrm{N}-[4-[[(2,4-\text { diamino-6- } \\
\text { pteridinyl)methyl]methylamino]benzoyl]- } & \text { [CAS] }\end{array}$ \\
\hline SAM001247043 & 1 & $\mathrm{H} 10$ & Tocris Bioscience & TFMPP \\
\hline SAM001247006 & 1 & $\mathrm{~A} 11$ & Tocris Bioscience & Pramipexole \\
\hline SAM001247018 & 1 & $\mathrm{~B} 11$ & Tocris Bioscience & LIDOCAINE \\
\hline SAM001246982 & 1 & $\mathrm{C} 11$ & Tocris Bioscience & Indomethacin \\
\hline SAM001246988 & 1 & $\mathrm{D} 11$ & Tocris Bioscience & LY 171883 \\
\hline SAM001246991 & 1 & $\mathrm{E} 11$ & Tocris Bioscience & Paroxetine \\
\hline SAM001247031 & 1 & $\mathrm{~F} 11$ & Tocris Bioscience & Epigallocatechin gallate \\
\hline SAM001247020 & 1 & G11 & Tocris Bioscience & 5-Amino-2-hydroxy-benzoic acid \\
\hline SAM001247025 & 1 & $\mathrm{H} 11$ & Tocris Bioscience & $\begin{array}{l}\text { Oxiranecarboxylic acid, 2-[6-(4-chlorophenoxy)hexyl]-, } \\
\text { ethyl ester- [CAS] }\end{array}$ \\
\hline SAM001246761 & 2 & $\mathrm{~A} 02$ & Sequoia RP Ltd & Cephalexin monohydrate \\
\hline SAM001246762 & 2 & $\mathrm{~B} 02$ & Sequoia RP Ltd & PIDOTIMOD \\
\hline SAM001246757 & 2 & $\mathrm{C} 02$ & Sequoia RP Ltd & RAMIPRIL \\
\hline SAM001246642 & 2 & D02 & Sequoia RP Ltd & FENPIVERINIUM BROMIDE \\
\hline SAM001246751 & 2 & E02 & Sequoia RP Ltd & \\
\hline SAM001246752 & 2 & $\mathrm{~F} 02$ & Sequoia RP Ltd & NIZATIDINE \\
\hline SAM001246753 & 2 & G02 & Sequoia RP Ltd & 5-FLUOROCYTOSINE \\
\hline SAM001246754 & 2 & $\mathrm{H} 02$ & Sequoia RP Ltd & Trileptal \\
\hline SAM001246756 & 2 & $\mathrm{~A} 03$ & Sequoia RP Ltd & TROXIPIDE \\
\hline SAM001246647 & 2 & $\mathrm{~B} 03$ & Sequoia RP Ltd & ACTARIT \\
\hline SAM001246645 & 2 & $\mathrm{C} 03$ & Sequoia RP Ltd & AZELASTINE HCl \\
\hline SAM001246759 & 2 & $\mathrm{D} 03$ & Sequoia RP Ltd & TOCAINIDE \\
\hline SAM001246760 & 2 & $\mathrm{E} 03$ & Sequoia RP Ltd & TAXIFOLIN-(+/-) \\
\hline SAM001246758 & 2 & F03 & Sequoia RP Ltd & LEVOFLOXACIN \\
\hline SAM001246643 & 2 & G03 & Sequoia RP Ltd & CEFATRIZINE PROPYLENE GLYCOL \\
\hline SAM001246700 & 2 & $\mathrm{H} 03$ & Sequoia RP Ltd & IDEBENONE \\
\hline SAM001246703 & 2 & $\mathrm{~A} 04$ & Sequoia RP Ltd & LEVOSULPIRIDE \\
\hline SAM001246706 & 2 & B04 & Sequoia RP Ltd & Pemoline \\
\hline SAM001246649 & 2 & $\mathrm{C} 04$ & Sequoia RP Ltd & LETROZOLE \\
\hline SAM001246650 & 2 & $\mathrm{D} 04$ & Sequoia RP Ltd & MEROPENEM \\
\hline SAM001246637 & 2 & E04 & Sequoia RP Ltd & ORLISTAT \\
\hline SAM001246631 & 2 & F04 & Sequoia RP Ltd & \\
\hline SAM001246694 & 2 & G04 & Sequoia RP Ltd & LEVONORGESTREL \\
\hline SAM001246695 & 2 & $\mathrm{H} 04$ & Sequoia RP Ltd & CETRAXATE HCl \\
\hline SAM001246696 & 2 & A05 & Sequoia RP Ltd & Alprazolam \\
\hline SAM001246697 & 2 & B05 & Sequoia RP Ltd & LAMOTRIGINE \\
\hline SAM001246702 & 2 & $\mathrm{C} 05$ & Sequoia RP Ltd & $\mathrm{N}$-Ethyl-o-crotonotoluidide \\
\hline SAM001246699 & 2 & $\mathrm{D} 05$ & Sequoia RP Ltd & AMFEBUTAMONE HCl \\
\hline SAM001246638 & 2 & E05 & Sequoia RP Ltd & ALFUZOSIN \\
\hline SAM001246639 & 2 & F05 & Sequoia RP Ltd & Amisulpride \\
\hline SAM001246632 & 2 & G05 & Sequoia RP Ltd & LOFEPRAMINE \\
\hline SAM001246688 & 2 & $\mathrm{H} 05$ & Sequoia RP Ltd & PEROSPIRONE HCl \\
\hline SAM001246689 & 2 & $\mathrm{~A} 06$ & Sequoia RP Ltd & DOCETAXEL \\
\hline SAM001246690 & 2 & B06 & Sequoia RP Ltd & HONOKIOL \\
\hline SAM001246691 & 2 & $\mathrm{C} 06$ & Sequoia RP Ltd & TOLTERODINE TARTRATE \\
\hline SAM001246692 & 2 & $\mathrm{D} 06$ & Sequoia RP Ltd & CARMOFUR \\
\hline SAM001246633 & 2 & E06 & Sequoia RP Ltd & PAROXETINE \\
\hline
\end{tabular}




\begin{tabular}{|c|c|c|c|c|}
\hline SAM001246634 & 2 & F06 & Sequoia RP Ltd & OLMESARTAN MEDOXOMIL \\
\hline SAM001246635 & 2 & G06 & Sequoia RP Ltd & LOSARTAN Potassium \\
\hline SAM001246636 & 2 & H06 & Sequoia RP Ltd & TEMOZOLOMIDE \\
\hline SAM001246682 & 2 & A07 & Sequoia RP Ltd & Methyltestosterone \\
\hline SAM001246686 & 2 & B07 & Sequoia RP Ltd & TOSUFLOXACIN TOSYLATE \\
\hline SAM001246687 & 2 & $\mathrm{C} 07$ & Sequoia RP Ltd & MECILLINAM \\
\hline SAM001246626 & 2 & D07 & Sequoia RP Ltd & ATOMOXETINE HCI \\
\hline SAM001246628 & 2 & E07 & Sequoia RP Ltd & ARTESUNATE \\
\hline SAM001246679 & 2 & F07 & Sequoia RP Ltd & ITRACONAZOLE \\
\hline SAM001246674 & 2 & G07 & Sequoia RP Ltd & CEFPODOXIME PROXETIL \\
\hline SAM001246680 & 2 & $\mathrm{H} 07$ & Sequoia RP Ltd & Buflomedil $\mathrm{HCl}$ \\
\hline SAM001246614 & 2 & A08 & Sequoia RP Ltd & 4-Chloro-N-(2-morpholin-4-yl-ethyl)-benzamide \\
\hline SAM001246616 & 2 & B08 & Sequoia RP Ltd & HALOMETASONE MONOHYDRATE \\
\hline SAM001246681 & 2 & $\mathrm{C} 08$ & Sequoia RP Ltd & TRICLABENDAZOLE \\
\hline SAM001246617 & 2 & D08 & Sequoia RP Ltd & ROFECOXIB \\
\hline SAM001246618 & 2 & E08 & Sequoia RP Ltd & BISOPROLOL FUMARATE \\
\hline SAM001246623 & 2 & F08 & Sequoia RP Ltd & EZETIMIBE \\
\hline SAM001246625 & 2 & G08 & Sequoia RP Ltd & TIAGABINE $\mathrm{HCl}$ \\
\hline SAM001246676 & 2 & H08 & Sequoia RP Ltd & IDARUBICIN HCl \\
\hline SAM001246685 & 2 & A09 & Sequoia RP Ltd & FLUBENDAZOLE \\
\hline SAM001246677 & 2 & B09 & Sequoia RP Ltd & TACROLIMUS \\
\hline SAM001246749 & 2 & $\mathrm{C} 09$ & Sequoia RP Ltd & VALACICLOVIR HYDROCHLORIDE \\
\hline SAM001246748 & 2 & D09 & Sequoia RP Ltd & CLARITHROMYCIN \\
\hline SAM001246750 & 2 & E09 & Sequoia RP Ltd & ARIPIPRAZOLE \\
\hline SAM001246747 & 2 & F09 & Sequoia RP Ltd & TRIMEBUTINE MALEATE \\
\hline SAM001246746 & 2 & G09 & Sequoia RP Ltd & Mestanolone \\
\hline SAM001246719 & 2 & H09 & Sequoia RP Ltd & NISOLDIPINE \\
\hline SAM001246720 & 2 & $\mathrm{~A} 10$ & Sequoia RP Ltd & PICEID \\
\hline SAM001246716 & 2 & $\mathrm{~B} 10$ & Sequoia RP Ltd & 1-(2-Methyl-5-nitro-imidazol-1-yl)-propan-2-ol \\
\hline SAM001246717 & 2 & $\mathrm{C} 10$ & Sequoia RP Ltd & NIFEKALANT $\mathrm{HCl}$ \\
\hline SAM001246721 & 2 & D10 & Sequoia RP Ltd & NATEGLINIDE \\
\hline SAM001246722 & 2 & E10 & Sequoia RP Ltd & MEGESTROL ACETATE \\
\hline SAM001246728 & 2 & F10 & Sequoia RP Ltd & ORMETOPRIM \\
\hline SAM001246738 & 2 & G10 & Sequoia RP Ltd & ZILEUTON \\
\hline SAM001246729 & 2 & $\mathrm{H} 10$ & Sequoia RP Ltd & STAVUDINE \\
\hline SAM001246730 & 2 & A11 & Sequoia RP Ltd & \\
\hline SAM001246724 & 2 & B11 & Sequoia RP Ltd & OXICONAZOLE NITRATE \\
\hline SAM001246731 & 2 & $\mathrm{C} 11$ & Sequoia RP Ltd & KITASAMYCIN \\
\hline SAM001246732 & 2 & D11 & Sequoia RP Ltd & FAMCICLOVIR \\
\hline SAM001246725 & 2 & E11 & Sequoia RP Ltd & \\
\hline SAM001246726 & 2 & F11 & Sequoia RP Ltd & RUFLOXACIN HCl \\
\hline SAM001246778 & 2 & G11 & Sequoia RP Ltd & TAXIFOLIN-(+) \\
\hline SAM001246782 & 2 & $\mathrm{H} 11$ & Sequoia RP Ltd & ALOSETRON HCl \\
\hline SAM001246723 & 3 & A02 & Sequoia RP Ltd & BUPROPION HYDROCHLORIDE \\
\hline SAM001246718 & 3 & B02 & Sequoia RP Ltd & IRSOGLADINE MALEATE \\
\hline SAM001246733 & 3 & $\mathrm{C} 02$ & Sequoia RP Ltd & ACARBOSE \\
\hline SAM001246739 & 3 & D02 & Sequoia RP Ltd & BENPROPERINE PHOSPHATE \\
\hline SAM001246740 & 3 & E02 & Sequoia RP Ltd & PHENPROBAMATE \\
\hline SAM001246743 & 3 & F02 & Sequoia RP Ltd & MEMANTINE HYDROCHLORIDE \\
\hline SAM001246736 & 3 & G02 & Sequoia RP Ltd & Carvedilol \\
\hline SAM001246741 & 3 & $\mathrm{H} 02$ & Sequoia RP Ltd & LOMIFYLLINE \\
\hline SAM001246742 & 3 & A03 & Sequoia RP Ltd & PAZUFLOXACIN \\
\hline
\end{tabular}




\begin{tabular}{|c|c|c|c|c|}
\hline SAM001246745 & 3 & $\mathrm{~B} 03$ & Sequoia RP Ltd & MIGLITOL \\
\hline SAM001246737 & 3 & $\mathrm{C} 03$ & Sequoia RP Ltd & TRANILAST \\
\hline SAM001246652 & 3 & D03 & Sequoia RP Ltd & OLANZAPINE \\
\hline SAM001246653 & 3 & E03 & Sequoia RP Ltd & Nefazodone \\
\hline SAM001246654 & 3 & F03 & Sequoia RP Ltd & MOXIFLOXACIN HCl \\
\hline SAM001246655 & 3 & G03 & Sequoia RP Ltd & NELFINAVIR MESYLATE \\
\hline SAM001246656 & 3 & $\mathrm{H} 03$ & Sequoia RP Ltd & PRAVASTATIN Sodium \\
\hline SAM001246651 & 3 & A04 & Sequoia RP Ltd & TOPOTECAN HCL \\
\hline SAM001246539 & 3 & B04 & Sequoia RP Ltd & LEVETIRACETAM \\
\hline SAM001246540 & 3 & $\mathrm{C} 04$ & Sequoia RP Ltd & PRAMIPEXOLE HCI \\
\hline SAM001246595 & 3 & D04 & Sequoia RP Ltd & RISPERIDONE \\
\hline SAM001246600 & 3 & E04 & Sequoia RP Ltd & PIOGLITAZONE HCI \\
\hline SAM001246552 & 3 & F04 & Sequoia RP Ltd & CILASTATIN Na \\
\hline SAM001246661 & 3 & G04 & Sequoia RP Ltd & ARGATROBAN \\
\hline SAM001246603 & 3 & $\mathrm{H} 04$ & Sequoia RP Ltd & VALDECOXIB \\
\hline SAM001246658 & 3 & A05 & Sequoia RP Ltd & NAFTOPIDIL \\
\hline SAM001246662 & 3 & B05 & Sequoia RP Ltd & Nobiletin \\
\hline SAM001246541 & 3 & $\mathrm{C} 05$ & Sequoia RP Ltd & FINASTERIDE \\
\hline SAM001246549 & 3 & D05 & Sequoia RP Ltd & ZOLPIDEM TARTRATE \\
\hline SAM001246551 & 3 & E05 & Sequoia RP Ltd & Viramune \\
\hline SAM001246601 & 3 & F05 & Sequoia RP Ltd & TOPIRAMATE \\
\hline SAM001246664 & 3 & G05 & Sequoia RP Ltd & VORICONAZOLE \\
\hline SAM001246665 & 3 & H05 & Sequoia RP Ltd & FENOLDOPAM MESYLATE \\
\hline SAM001246610 & 3 & A06 & Sequoia RP Ltd & ROSIGLITAZONE MALEATE \\
\hline SAM001246668 & 3 & B06 & Sequoia RP Ltd & ESCITALOPRAM OXALATE \\
\hline SAM001246609 & 3 & $\mathrm{C} 06$ & Sequoia RP Ltd & ZERANOL \\
\hline SAM001246671 & 3 & D06 & Sequoia RP Ltd & LATANOPROST \\
\hline SAM001246673 & 3 & E06 & Sequoia RP Ltd & 2',3'-DIDEOXYINOSINE \\
\hline SAM001246666 & 3 & F06 & Sequoia RP Ltd & Sertraline \\
\hline SAM001246670 & 3 & G06 & Sequoia RP Ltd & CALCIPOTRIOL \\
\hline SAM001246559 & 3 & $\mathrm{H} 06$ & Sequoia RP Ltd & EPIRUBICIN HYDROCHLORIDE \\
\hline SAM001246612 & 3 & A07 & Sequoia RP Ltd & BICALUTAMIDE \\
\hline SAM001246672 & 3 & B07 & Sequoia RP Ltd & BENIDIPINE $\mathrm{HCl}$ \\
\hline SAM001246669 & 3 & $\mathrm{C} 07$ & Sequoia RP Ltd & AMLEXANOX \\
\hline SAM001246554 & 3 & D07 & Sequoia RP Ltd & CERIVASTATIN Na \\
\hline SAM001246560 & 3 & E07 & Sequoia RP Ltd & ICARIIN \\
\hline SAM001246562 & 3 & F07 & Sequoia RP Ltd & METHYLANDROSTENEDIOL \\
\hline SAM001246553 & 3 & G07 & Sequoia RP Ltd & TRIPTOLIDE \\
\hline SAM001246608 & 3 & $\mathrm{H} 07$ & Sequoia RP Ltd & ROSIGLITAZONE HCl \\
\hline SAM001246561 & 3 & $\mathrm{~A} 08$ & Sequoia RP Ltd & FTORAFUR \\
\hline SAM001246769 & 3 & B08 & Sequoia RP Ltd & OLIGOMYCIN C \\
\hline SAM001246712 & 3 & $\mathrm{C} 08$ & Sequoia RP Ltd & BENAZEPRIL HCl \\
\hline SAM001246765 & 3 & D08 & Sequoia RP Ltd & Oxymetholone \\
\hline SAM001246714 & 3 & E08 & Sequoia RP Ltd & IPRIFLAVONE \\
\hline SAM001246770 & 3 & F08 & Sequoia RP Ltd & OXAPROZIN \\
\hline SAM001246766 & 3 & G08 & Sequoia RP Ltd & ROLIPRAM \\
\hline SAM001246713 & 3 & H08 & Sequoia RP Ltd & MOSAPRIDE CITRATE \\
\hline SAM001246767 & 3 & A09 & Sequoia RP Ltd & Isoquercitrin \\
\hline SAM001246763 & 3 & B09 & Sequoia RP Ltd & FLUMAZENIL \\
\hline SAM001246593 & 3 & $\mathrm{Co9}$ & Sequoia RP Ltd & \\
\hline SAM001246776 & 3 & D09 & Sequoia RP Ltd & HYPEROSIDE \\
\hline SAM001246589 & 3 & E09 & Sequoia RP Ltd & RIFABUTIN \\
\hline
\end{tabular}




\begin{tabular}{|c|c|c|c|c|}
\hline SAM001246533 & 3 & F09 & Sequoia RP Ltd & ESMOLOL HYDROCHLORIDE \\
\hline SAM001246586 & 3 & G09 & Sequoia RP Ltd & TADALAFIL \\
\hline SAM001246587 & 3 & $\mathrm{H} 09$ & Sequoia RP Ltd & Modafinil \\
\hline SAM001246768 & 3 & A10 & Sequoia RP Ltd & DOXORUBICIN HYDROCHLORIDE \\
\hline SAM001246764 & 3 & B10 & Sequoia RP Ltd & MOXONIDINE HCl \\
\hline SAM001246711 & 3 & $\mathrm{C} 10$ & Sequoia RP Ltd & Nitrazepam \\
\hline SAM001246571 & 3 & D10 & Sequoia RP Ltd & PEFLOXACIN MESYLATE \\
\hline SAM001246572 & 3 & E10 & Sequoia RP Ltd & VENLAFAXINE HCl \\
\hline SAM001246591 & 3 & F10 & Sequoia RP Ltd & PANTOPRAZOLE SODIUM SALT \\
\hline SAM001246583 & 3 & G10 & Sequoia RP Ltd & FLUTICASONE PROPIONATE \\
\hline SAM001246588 & 3 & $\mathrm{H} 10$ & Sequoia RP Ltd & INDINAVIR SULPHATE \\
\hline SAM001246585 & 3 & A11 & Sequoia RP Ltd & MIDAZOLAM HCl \\
\hline SAM001246582 & 3 & B11 & Sequoia RP Ltd & LAMIVUDINE \\
\hline SAM001246564 & 3 & $\mathrm{C} 11$ & Sequoia RP Ltd & PROCARBAZINE HYDROCHLORIDE \\
\hline SAM001246538 & 3 & D11 & Sequoia RP Ltd & ESOMEPRAZOLE Mg \\
\hline SAM001246530 & 3 & E11 & Sequoia RP Ltd & SULFASALAZINE \\
\hline SAM001246567 & 3 & $\mathrm{~F} 11$ & Sequoia RP Ltd & TORASEMIDE \\
\hline SAM001246576 & 3 & G11 & Sequoia RP Ltd & TROPISETRON HCI \\
\hline SAM001246534 & 3 & $\mathrm{H} 11$ & Sequoia RP Ltd & Ranolazine dihydrochloride \\
\hline SAM001246531 & 4 & A02 & Sequoia RP Ltd & NITRENDIPINE \\
\hline SAM001246578 & 4 & B02 & Sequoia RP Ltd & SAQUINAVIR MESYLATE \\
\hline SAM001246775 & 4 & $\mathrm{C} 02$ & Sequoia RP Ltd & BIFONAZOLE \\
\hline SAM001246579 & 4 & D02 & Sequoia RP Ltd & SUMATRIPTAN SUCCINATE \\
\hline SAM001246574 & 4 & E02 & Sequoia RP Ltd & EXEMESTANE \\
\hline SAM001246708 & 4 & F02 & Sequoia RP Ltd & NITAZOXANIDE \\
\hline SAM001246536 & 4 & G02 & Sequoia RP Ltd & Diazepam \\
\hline SAM001246777 & 4 & H02 & Sequoia RP Ltd & QUETIAPINE HEMIFUMARATE \\
\hline SAM001246528 & 4 & $\mathrm{~A} 03$ & Sequoia RP Ltd & RUTIN \\
\hline SAM001246580 & 4 & $\mathrm{~B} 03$ & Sequoia RP Ltd & PENCICLOVIR \\
\hline SAM001246772 & 4 & $\mathrm{C} 03$ & Sequoia RP Ltd & CALCITRIOL \\
\hline SAM001246532 & 4 & D03 & Sequoia RP Ltd & DIPHENOXYLATE \\
\hline SAM001247005 & 4 & E03 & Tocris Bioscience & Felbamate \\
\hline SAM001247013 & 4 & F03 & Tocris Bioscience & DROPERIDOL \\
\hline SAM001247011 & 4 & G03 & Tocris Bioscience & Pentoxifylline \\
\hline SAM001246596 & 4 & $\mathrm{H} 03$ & Sequoia RP Ltd & \\
\hline SAM001246783 & 4 & A04 & Sequoia RP Ltd & RITONAVIR \\
\hline SAM001246780 & 4 & B04 & Sequoia RP Ltd & VINORELBINE BITATRATE \\
\hline SAM001246624 & 4 & $\mathrm{C} 04$ & Sequoia RP Ltd & LINEZOLID \\
\hline SAM001246727 & 4 & D04 & Sequoia RP Ltd & LOMERIZINE DiHCI \\
\hline SAM001246667 & 4 & E04 & Sequoia RP Ltd & EFAVIRENZ \\
\hline SAM001246548 & 4 & F04 & Sequoia RP Ltd & IRBESARTAN \\
\hline SAM001246546 & 4 & G04 & Sequoia RP Ltd & REPAGLINIDE \\
\hline SAM001246555 & 4 & $\mathrm{H} 04$ & Sequoia RP Ltd & Ethylestrenol \\
\hline SAM001246605 & 4 & A05 & Sequoia RP Ltd & PTEROSTILBENE \\
\hline SAM001246547 & 4 & B05 & Sequoia RP Ltd & ROXATIDINE ACETATE $\mathrm{HCl}$ \\
\hline SAM001246556 & 4 & $\mathrm{C} 05$ & Sequoia RP Ltd & DEXBROMPHENIRAMINE MALEATE \\
\hline SAM001246604 & 4 & D05 & Sequoia RP Ltd & ANAGRELIDE $\mathrm{HCl}$ \\
\hline SAM001246606 & 4 & E05 & Sequoia RP Ltd & TEGASEROD MALEATE \\
\hline SAM001246611 & 4 & F05 & Sequoia RP Ltd & MILRINONE \\
\hline SAM001246575 & 4 & G05 & Sequoia RP Ltd & LEVOCETIRIZINE \\
\hline SAM001246599 & 4 & $\mathrm{H} 05$ & Sequoia RP Ltd & \\
\hline SAM001246558 & 4 & A06 & Sequoia RP Ltd & TICLOPIDINE HCI \\
\hline
\end{tabular}




\begin{tabular}{|c|c|c|c|c|}
\hline SAM001246594 & 4 & B06 & Sequoia RP Ltd & LOXOPROFEN SODIUM \\
\hline SAM001246577 & 4 & $\mathrm{C} 06$ & Sequoia RP Ltd & ZAFIRLUKAST \\
\hline SAM001246565 & 4 & $\mathrm{D} 06$ & Sequoia RP Ltd & TERBINAFINE HCl \\
\hline SAM001246584 & 4 & E06 & Sequoia RP Ltd & ISRADIPINE \\
\hline SAM001246581 & 4 & F06 & Sequoia RP Ltd & VALSARTAN \\
\hline SAM001247048 & 4 & G06 & Tocris Bioscience & Piroxicam \\
\hline SAM001246629 & 4 & $\mathrm{H} 06$ & Sequoia RP Ltd & Glycopyrrolate \\
\hline SAM001246992 & 4 & $\mathrm{~A} 07$ & Tocris Bioscience & Physostigmine \\
\hline SAM001247050 & 4 & B07 & Tocris Bioscience & LOBELINE HYDROCHLORIDE \\
\hline SAM001247030 & 4 & $\mathrm{C07}$ & Tocris Bioscience & Doxylamine succinate salt \\
\hline SAM001247035 & 4 & $\mathrm{D} 07$ & Tocris Bioscience & Milnacipran \\
\hline SAM001247017 & 4 & E07 & Tocris Bioscience & 5-fluoro-2-pyrimidone \\
\hline SAM001247022 & 4 & $\mathrm{~F} 07$ & Tocris Bioscience & Chlorpheniramine \\
\hline SAM001246621 & 4 & G07 & Sequoia RP Ltd & DOFETILIDE \\
\hline SAM001246675 & 4 & $\mathrm{H} 07$ & Sequoia RP Ltd & FORMOTEROL FUMARATE DIHYDRATE \\
\hline SAM001246615 & 4 & A08 & Sequoia RP Ltd & RIZATRIPTAN BENZOATE \\
\hline SAM001246620 & 4 & B08 & Sequoia RP Ltd & RIFAPENTINE \\
\hline SAM001246630 & 4 & $\mathrm{C} 08$ & Sequoia RP Ltd & LOTEPREDNOL ETABONATE \\
\hline SAM001246684 & 4 & D08 & Sequoia RP Ltd & ENALAPRILAT \\
\hline SAM001246627 & 4 & E08 & Sequoia RP Ltd & Donepezil \\
\hline SAM001246755 & 4 & F08 & Sequoia RP Ltd & Nimetazepam \\
\hline SAM001246701 & 4 & G08 & Sequoia RP Ltd & \\
\hline SAM001246602 & 4 & $\mathrm{H} 08$ & Sequoia RP Ltd & TELMISARTAN \\
\hline SAM001246542 & 4 & A09 & Sequoia RP Ltd & ITOPRIDE HCl \\
\hline SAM001246597 & 4 & B09 & Sequoia RP Ltd & RIFAXIMIN \\
\hline SAM001246657 & 4 & $\mathrm{Cog}$ & Sequoia RP Ltd & MONTELUKAST Na \\
\hline SAM001246779 & 4 & D09 & Sequoia RP Ltd & 2',3'-DIDEOXYCYTIDINE \\
\hline SAM001247073 & 4 & E09 & Tocris Bioscience & $\begin{array}{l}\text { 1H-Imidazol-2-amine, } \quad \mathrm{N} \text {-(2,6-dichlorophenyl)-4,5- } \\
\text { dihydro- [CAS] }\end{array}$ \\
\hline SAM001247077 & 4 & F09 & Tocris Bioscience & $\begin{array}{l}\text { 6H-Pyrido[2,3-b][1,4]benzodiazepin-6-one, } \\
{[(\text { diethylamino)methyl]-1-piperidinyl]acetyl]-5,11-dihydro- }} \\
{[\mathrm{CAS}]}\end{array}$ \\
\hline SAM001247075 & 4 & G09 & Tocris Bioscience & $\begin{array}{l}\text { 1H-Indole-2-propanoic acid, 1-[(4-chlorophenyl)methyl]- } \\
\text { 3-[(1,1-dimethylethyl)thio]-Alpha,Alpha-dimethyl-5-(1- } \\
\text { methylethyl)- [CAS] }\end{array}$ \\
\hline SAM001247074 & 4 & $\mathrm{H} 09$ & Tocris Bioscience & $\begin{array}{l}\text { 1H-Imidazole-5-carboxylic acid, 1-(1-phenylethyl)-, ethyl } \\
\text { ester, (R)- [CAS] }\end{array}$ \\
\hline SAM001247078 & 4 & $\mathrm{~A} 10$ & Tocris Bioscience & $\begin{array}{l}\text { Acetamide, 2-amino-N-(1-methyl-1,2-diphenylethyl)-, (+/- } \\
) \text { - [CAS] }\end{array}$ \\
\hline SAM001247080 & 4 & $\mathrm{~B} 10$ & Tocris Bioscience & Altanserin \\
\hline SAM001247084 & 4 & $\mathrm{C} 10$ & Tocris Bioscience & \\
\hline SAM001246563 & 4 & D10 & Sequoia RP Ltd & \\
\hline SAM001247082 & 4 & E10 & Tocris Bioscience & Azasetron \\
\hline SAM001247087 & 4 & $\mathrm{~F} 10$ & Tocris Bioscience & GR 89696 \\
\hline SAM001246897 & 4 & G10 & Sigma CC & $\begin{array}{l}\text { DELTA1-HYDROCORTISONE } \\
\text { SODIUM SALT }\end{array}$ \\
\hline SAM001246872 & 4 & $\mathrm{H} 10$ & Sigma CC & DIAZOXIDE \\
\hline SAM001246886 & 4 & A11 & Sigma CC & 2-CHLOROADENOSINE \\
\hline SAM001246873 & 4 & $\mathrm{~B} 11$ & Sigma CC & ORNIDAZOLE \\
\hline SAM001246914 & 4 & $\mathrm{C} 11$ & Sigma CC & 1,1-DIMETHYL-4-PHENYLPIPERAZINIUM IODIDE \\
\hline SAM001246893 & 4 & $\mathrm{D} 11$ & Sigma CC & PIRENPERONE \\
\hline SAM001246866 & 4 & E11 & Sigma CC & MESTRANOL \\
\hline SAM001246867 & 4 & $\mathrm{~F} 11$ & Sigma CC & 2-(2-AMINOETHYL)PYRIDINE \\
\hline SAM001246868 & 4 & G11 & Sigma CC & BENACTYZINE HYDROCHLORIDE \\
\hline SAM001246870 & 4 & $\mathrm{H} 11$ & Sigma CC & DICHLOROACETIC ACID \\
\hline SAM001246806 & 5 & $\mathrm{~A} 02$ & Sequoia RP Ltd & BESTATIN \\
\hline
\end{tabular}




\begin{tabular}{|c|c|c|c|c|}
\hline SAM001246774 & 5 & $\mathrm{~B} 02$ & Sequoia RP Ltd & TOREMIFENE CITRATE \\
\hline SAM001246784 & 5 & $\mathrm{C} 02$ & Sequoia RP Ltd & GOSERELIN ACETATE \\
\hline SAM001246678 & 5 & D02 & Sequoia RP Ltd & SECOISOLARICIRESINOL \\
\hline SAM001246796 & 5 & E02 & Sequoia RP Ltd & RALTITREXED \\
\hline SAM001246792 & 5 & $\mathrm{~F} 02$ & Sequoia RP Ltd & DOXAPRAM HYDROCHLORIDE \\
\hline SAM001247095 & 5 & G02 & Tocris Bioscience & RU 24969 \\
\hline SAM001246865 & 5 & $\mathrm{H} 02$ & Sigma CC & Brucine \\
\hline SAM001246862 & 5 & $\mathrm{~A} 03$ & Sigma CC & TRYPTOLINE \\
\hline SAM001246863 & 5 & B03 & Sigma CC & \\
\hline SAM001246791 & 5 & $\mathrm{CO3}$ & Sequoia RP Ltd & PALONOSETRON HCl \\
\hline SAM001246874 & 5 & $\mathrm{D} 03$ & Sigma CC & NAPROXEN SODIUM \\
\hline SAM001246875 & 5 & E03 & Sigma CC & MEPIVACAINE HYDROCHLORIDE \\
\hline SAM001246877 & 5 & F03 & Sigma CC & $\begin{array}{l}\text { 3-[3,5-DIBROMO-4-HYDROXYBENZOYL]-2- } \\
\text { ETHYLBENZOFURAN }\end{array}$ \\
\hline SAM001246890 & 5 & G03 & Sigma CC & NIMODIPINE \\
\hline SAM001246892 & 5 & $\mathrm{H} 03$ & Sigma CC & ROLITETRACYCLINE \\
\hline SAM001246891 & 5 & $\mathrm{~A} 04$ & Sigma CC & MEPIRIZOLE \\
\hline SAM001246876 & 5 & B04 & Sigma CC & 6-AZAURIDINE \\
\hline SAM001246889 & 5 & $\mathrm{CO} 4$ & Sigma CC & Reichsteins substance $\square \mathrm{S}$ \\
\hline SAM001246894 & 5 & D04 & Sigma CC & 3-PYRIDINEMETHANOL \\
\hline SAM001246979 & 5 & E04 & Tocris Bioscience & Haloperidol \\
\hline SAM001247042 & 5 & F04 & Tocris Bioscience & Stiripentol \\
\hline SAM001247034 & 5 & G04 & Tocris Bioscience & Fluperlapine \\
\hline SAM001246887 & 5 & $\mathrm{H} 04$ & Sigma CC & OXYPHENONIUM BROMIDE \\
\hline SAM001246869 & 5 & $\mathrm{~A} 05$ & Sigma CC & Homoveratrylamine \\
\hline SAM001246913 & 5 & B05 & Sigma CC & TINIDAZOLE \\
\hline SAM001246912 & 5 & $\mathrm{C} 05$ & Sigma CC & XANTHINOL NICOTINATE \\
\hline SAM001246896 & 5 & $\mathrm{D} 05$ & Sigma CC & SYNEPHRINE \\
\hline SAM001246888 & 5 & E05 & Sigma CC & Resveratrol \\
\hline SAM001246871 & 5 & F05 & Sigma CC & 3-Hydroxy-2-methyl-4-pyrone \\
\hline SAM001246854 & 5 & G05 & Sigma CC & 6-AMINOINDAZOLE \\
\hline SAM001246856 & 5 & $\mathrm{H} 05$ & Sigma CC & ENROFLOXACIN \\
\hline SAM001246857 & 5 & $\mathrm{~A} 06$ & Sigma CC & DEHYDROCHOLIC ACID \\
\hline SAM001246884 & 5 & B06 & Sigma CC & CEFACLOR \\
\hline SAM001246858 & 5 & $\mathrm{C} 06$ & Sigma CC & 1-BENZYLIMIDAZOLE \\
\hline SAM001246523 & 5 & $\mathrm{D} 06$ & Sequoia RP Ltd & DULOXETINE HCl \\
\hline SAM001246573 & 5 & E06 & Sequoia RP Ltd & VARDENAFIL CITRATE \\
\hline SAM001246524 & 5 & F06 & Sequoia RP Ltd & ROPIVACAINE HCl \\
\hline SAM001246525 & 5 & G06 & Sequoia RP Ltd & ANASTROZOLE \\
\hline SAM001246984 & 5 & $\mathrm{H} 06$ & Tocris Bioscience & KETOTIFEN FUMARATE \\
\hline SAM001246906 & 5 & $\mathrm{~A} 07$ & Sigma CC & MEDROXYPROGESTERONE \\
\hline SAM001246907 & 5 & B07 & Sigma CC & Pinacidil monohydrate \\
\hline SAM001246908 & 5 & $\mathrm{C} 07$ & Sigma CC & 7-NITROINDAZOLE \\
\hline SAM001246909 & 5 & D07 & Sigma CC & 5-Methoxytryptamine \\
\hline SAM001246855 & 5 & E07 & Sigma CC & PHENOTHIAZINE \\
\hline SAM001246526 & 5 & $\mathrm{~F} 07$ & Sequoia RP Ltd & 2-CHLORO-2'-DEOXYADENOSINE \\
\hline SAM001246527 & 5 & G07 & Sequoia RP Ltd & GRANISETRON HCl \\
\hline SAM001247094 & 5 & $\mathrm{H} 07$ & Tocris Bioscience & Rimcazole \\
\hline SAM001247092 & 5 & $\mathrm{~A} 08$ & Tocris Bioscience & Nafadotride \\
\hline SAM001246885 & 5 & B08 & Sigma CC & DESOXIMETASONE \\
\hline SAM001246557 & 5 & $\mathrm{C} 08$ & Sequoia RP Ltd & DEXCHLORPHENIRAMINE MALEATE \\
\hline SAM001247088 & 5 & D08 & Tocris Bioscience & $\begin{array}{l}\text { Guanidine, } \\
\text { pyridinyl- [CAS] }\end{array} \quad$-cyano-N'-(1,1-dimethylpropyl)-N"-3- \\
\hline
\end{tabular}


Apendix

\begin{tabular}{|c|c|c|c|c|}
\hline SAM001247090 & 5 & E08 & Tocris Bioscience & $\mathrm{L}-694,247$ \\
\hline SAM001247081 & 5 & F08 & Tocris Bioscience & AM-251 \\
\hline SAM001247089 & 5 & G08 & Tocris Bioscience & HTMT \\
\hline SAM001247083 & 5 & $\mathrm{H} 08$ & Tocris Bioscience & $\begin{array}{l}\text { Benzo[a]phenanthridine-10,11-diol, } \\
\text { hexahydro-, trans- [CAS] }\end{array}$ \\
\hline SAM001247091 & 5 & A09 & Tocris Bioscience & $\begin{array}{l}\text { Methanesulfonamide, } \quad \mathrm{N}-[4-[[1-[2-(6-m e t h y l-2- \\
\text { pyridinyl)ethyl]-4-piperidinyl]carbonyl]phenyl]-, } \\
\text { dihydrochloride [CAS] }\end{array}$ \\
\hline SAM001247076 & 5 & B09 & Tocris Bioscience & $\begin{array}{l}2 \mathrm{H} \text {-Indol-2-one, } \\
\text { pyridinylmethyl)- [CAS] }\end{array}$ \\
\hline SAM001246898 & 5 & $\mathrm{Cog}$ & Sigma CC & Beclomethasone \\
\hline SAM001246900 & 5 & D09 & Sigma CC & OMEPRAZOLE \\
\hline SAM001246842 & 5 & E09 & Sequoia RP Ltd & DOLASETRON MESYLATE \\
\hline SAM001246841 & 5 & F09 & Sequoia RP Ltd & Zolmitriptan \\
\hline SAM001246852 & 5 & G09 & Sequoia RP Ltd & TREMULACIN \\
\hline SAM001246846 & 5 & $\mathrm{H} 09$ & Sequoia RP Ltd & DACTINOMYCIN \\
\hline SAM001246847 & 5 & $\mathrm{~A} 10$ & Sequoia RP Ltd & Tramadol \\
\hline SAM001246815 & 5 & $\mathrm{~B} 10$ & Sequoia RP Ltd & CHLORDIAZEPOXIDE \\
\hline SAM001246816 & 5 & $\mathrm{C} 10$ & Sequoia RP Ltd & CEFIXIME TRIHYDRATE \\
\hline SAM001246818 & 5 & $\mathrm{D} 10$ & Sequoia RP Ltd & CEFDINIR \\
\hline SAM001246820 & 5 & $\mathrm{E} 10$ & Sequoia RP Ltd & LOFEXIDINE HCl \\
\hline SAM001246804 & 5 & $\mathrm{~F} 10$ & Sequoia RP Ltd & BALSALAZIDE \\
\hline SAM001246802 & 5 & G10 & Sequoia RP Ltd & OLOPATADINE HCl \\
\hline SAM001246803 & 5 & $\mathrm{H} 10$ & Sequoia RP Ltd & ITAVASTATIN Ca \\
\hline SAM001246882 & 5 & A11 & Sigma CC & CORTISONE \\
\hline SAM001246883 & 5 & $\mathrm{~B} 11$ & Sigma CC & \\
\hline SAM001246822 & 5 & $\mathrm{C} 11$ & Sequoia RP Ltd & HOMOHARRINGTONINE \\
\hline SAM001246879 & 5 & D11 & Sigma CC & CORTICOSTERONE \\
\hline SAM001246821 & 5 & $\mathrm{E} 11$ & Sequoia RP Ltd & VECURONIUM BROMIDE \\
\hline SAM001246801 & 5 & $\mathrm{~F} 11$ & Sequoia RP Ltd & TIBOLONE \\
\hline SAM001246860 & 5 & G11 & Sigma CC & NICOTINAMIDE \\
\hline SAM001246861 & 5 & $\mathrm{H} 11$ & Sigma CC & NIALAMIDE \\
\hline SAM001246568 & 6 & $\mathrm{~A} 02$ & Sequoia RP Ltd & VINDESINE SULFATE \\
\hline SAM001246570 & 6 & B02 & Sequoia RP Ltd & VINCRISTINE SULFATE \\
\hline SAM001246648 & 6 & $\mathrm{C02}$ & Sequoia RP Ltd & LACIDIPINE \\
\hline SAM001246659 & 6 & $\mathrm{D} 02$ & Sequoia RP Ltd & MIRTAZAPINE \\
\hline SAM001246707 & 6 & E02 & Sequoia RP Ltd & AMPIROXICAM \\
\hline SAM001246710 & 6 & $\mathrm{~F} 02$ & Sequoia RP Ltd & GLIMEPIRIDE \\
\hline SAM001246705 & 6 & G02 & Sequoia RP Ltd & AMLODIPINE BASE \\
\hline SAM001246619 & 6 & $\mathrm{H} 02$ & Sequoia RP Ltd & RABEPRAZOLE \\
\hline SAM001246878 & 6 & $\mathrm{~A} 03$ & Sigma CC & CLOFAZIMINE \\
\hline SAM001246598 & 6 & B03 & Sequoia RP Ltd & IRINOTECAN HCl )trihydrate) \\
\hline SAM001246544 & 6 & $\mathrm{CO3}$ & Sequoia RP Ltd & LANSOPRAZOLE \\
\hline SAM001246545 & 6 & D03 & Sequoia RP Ltd & $\begin{array}{l}\text { 8-Chloro-11-piperidin-4-ylidene-6,11-dihydro-5H- } \\
\text { benzo[5,6]cyclohepta[1,2-b]pyridine }\end{array}$ \\
\hline SAM001246904 & 6 & E03 & Sigma CC & $\begin{array}{l}\text { 1,3,5(10)-ESTRATRIEN-3-OL-17-ONE } \\
\text { SODIUM SALT }\end{array}$ \\
\hline SAM001246881 & 6 & F03 & Sigma CC & MIFEPRISTONE \\
\hline SAM001246880 & 6 & G03 & Sigma CC & \\
\hline SAM001247105 & 6 & $\mathrm{HO3}$ & Tocris Bioscience & Sibutramine \\
\hline SAM001247107 & 6 & A04 & Tocris Bioscience & Clobenpropit \\
\hline SAM001246851 & 6 & B04 & Sequoia RP Ltd & HUPERZINE A \\
\hline SAM001246592 & 6 & $\mathrm{CO4}$ & Sequoia RP Ltd & SIBUTRAMINE HCl \\
\hline SAM001246833 & 6 & D04 & Sequoia RP Ltd & Lorazepam \\
\hline SAM001247108 & 6 & E04 & Tocris Bioscience & $\begin{array}{l}\text { 8-Azaspiro[4.5]decane-7,9-dione, } 8-[2-[[(2,3-d i h y d r o-1,4 \\
\text { benzodioxin-2-yl)methyl]amino]ethyl]-, }\end{array}$ \\
\hline
\end{tabular}


Apendix

\begin{tabular}{|c|c|c|c|c|}
\hline & & & & monomethanesulfonate [CAS] \\
\hline SAM001247106 & 6 & F04 & Tocris Bioscience & Adenosine, N-(2-hydroxycyclopentyl)-, (1S-trans)- [CAS] \\
\hline SAM001246646 & 6 & G04 & Sequoia RP Ltd & AMIODARONE HYDROCHLORIDE \\
\hline SAM001246644 & 6 & $\mathrm{H} 04$ & Sequoia RP Ltd & mevastatin \\
\hline SAM001246622 & 6 & A05 & Sequoia RP Ltd & IMATINIB MESYLATE \\
\hline SAM001247102 & 6 & B05 & Tocris Bioscience & Metylperon \\
\hline SAM001246773 & 6 & $\mathrm{C} 05$ & Sequoia RP Ltd & PARECOXIB Na \\
\hline SAM001247070 & 6 & D05 & Tocris Bioscience & \\
\hline SAM001246793 & 6 & E05 & Sequoia RP Ltd & ATRACURIUM BESYLATE \\
\hline SAM001246799 & 6 & F05 & Sequoia RP Ltd & ARTEMETHER \\
\hline SAM001247071 & 6 & G05 & Tocris Bioscience & EBSELEN \\
\hline SAM001247066 & 6 & $\mathrm{H} 05$ & Tocris Bioscience & CGS 12066B \\
\hline SAM001246805 & 6 & $\mathrm{~A} 06$ & Sequoia RP Ltd & TELITHROMYCIN \\
\hline SAM001247065 & 6 & B06 & Tocris Bioscience & CCPA \\
\hline SAM001247067 & 6 & $\mathrm{C} 06$ & Tocris Bioscience & PD 81723 \\
\hline SAM001246590 & 6 & D06 & Sequoia RP Ltd & Stanozolol \\
\hline SAM001246641 & 6 & E06 & Sequoia RP Ltd & Zaleplon \\
\hline SAM001246840 & 6 & F06 & Sequoia RP Ltd & Prostaglandin E1 \\
\hline SAM001246921 & 6 & G06 & Tocris Bioscience & Testosterone \\
\hline SAM001246922 & 6 & H06 & Tocris Bioscience & DEHYDROEPIANDROSTERONE \\
\hline SAM001247099 & 6 & $\mathrm{~A} 07$ & Tocris Bioscience & SDM25N \\
\hline SAM001247101 & 6 & B07 & Tocris Bioscience & $\begin{array}{l}\text { Thiophene, } \\
\text { (methylsulfonyl)phenyl]- [CAS] }\end{array}$ \\
\hline SAM001247103 & 6 & $\mathrm{C} 07$ & Tocris Bioscience & 5-Nonyloxytryptamine \\
\hline SAM001247096 & 6 & D07 & Tocris Bioscience & Salmeterol \\
\hline SAM001247097 & 6 & E07 & Tocris Bioscience & SB 205607 \\
\hline SAM001247098 & 6 & F07 & Tocris Bioscience & $\mathrm{R}(+)-\mathrm{SCH}-23390$ hydrochloride \\
\hline
\end{tabular}

Table 10. NCC003 


\section{Literature}

Abigerges, D., Chabot, G.G., Armand, J.P., Hérait, P., Gouyette, A., and Gandia, D., 1995, Phase I and pharmacologic studies of the camptothecin analog irinotecan administered every 3 weeks in cancer patients: Journal of clinical oncology: official journal of the American Society of Clinical Oncology, v. 13, no. 1, p. 210-221.

Adlkofer, K., and Lai, C., 2000, Role of neuregulins in glial cell development: Glia, v. 29, no. 2, p. 104-111.

Akbarian, S., Kim, J.J., Potkin, S.G., Hagman, J.O., Tafazzoli, A., Bunney, W.E., Jr, and Jones, E.G., 1995, Gene expression for glutamic acid decarboxylase is reduced without loss of neurons in prefrontal cortex of schizophrenics: Archives of general psychiatry, v. 52, no. 4, p. 258-266.

Aleman, A., Kahn, R.S., and Selten, J.-P., 2003, Sex differences in the risk of schizophrenia: evidence from meta-analysis: Archives of general psychiatry, v. 60, no. 6, p. 565-571, doi: 10.1001/archpsyc.60.6.565.

Alimonti, A., Gelibter, A., Pavese, I., Satta, F., Cognetti, F., Ferretti, G., Rasio, D., Vecchione, A., and Di Palma, M., 2004, New approaches to prevent intestinal toxicity of irinotecan-based regimens: Cancer treatment reviews, v. 30 , no. 6 , p. 555-562, doi: 10.1016/j.ctrv.2004.05.002.

Allen, N.C., Bagade, S., McQueen, M.B., Ioannidis, J.P.A., Kavvoura, F.K., Khoury, M.J., Tanzi, R.E., and Bertram, L., 2008, Systematic meta-analyses and field synopsis of genetic association studies in schizophrenia: the SzGene database: Nature genetics, v. 40 , no. 7 , p. $827-834$, doi: $10.1038 /$ ng. 171 .

Allen, R.M., and Young, S.J., 1978, Phencyclidine-induced psychosis: The American journal of psychiatry, v. 135 , no. 9, p. 1081-1084.

Arkin, M.R., and Wells, J.A., 2004, Small-molecule inhibitors of protein-protein interactions: progressing towards the dream: Nature Reviews Drug Discovery, v. 3, no. 4, p. 301317, doi: $10.1038 / \mathrm{nrd} 1343$.

Arnold, S.E., and Trojanowski, J.Q., 1996, Recent advances in defining the neuropathology of schizophrenia: Acta neuropathologica, v. 92, no. 3, p. 217-231.

Arnt, J., 1998, Pharmacological differentiation of classical and novel antipsychotics: International clinical psychopharmacology, v. 13 Suppl 3, p. S7-14.

Auger, K.R., Serunian, L.A., Soltoff, S.P., Libby, P., and Cantley, L.C., 1989, PDGFdependent tyrosine phosphorylation stimulates production of novel polyphosphoinositides in intact cells: Cell, v. 57, no. 1, p. 167-175.

Ayhan, Y., Sawa, A., Ross, C.A., and Pletnikov, M.V., 2009, Animal models of geneenvironment interactions in schizophrenia: Behavioural brain research, v. 204, no. 2, p. 274-281, doi: 10.1016/j.bbr.2009.04.010. 
Barr, M.S., Farzan, F., Tran, L.C., Chen, R., Fitzgerald, P.B., and Daskalakis, Z.J., 2010, Evidence for excessive frontal evoked gamma oscillatory activity in schizophrenia during working memory: Schizophrenia research, v. 121, no. 1-3, p. 146-152, doi: 10.1016/j.schres.2010.05.023.

de Bartolomeis, A., Sarappa, C., Magara, S., and lasevoli, F., 2012, Targeting glutamate system for novel antipsychotic approaches: relevance for residual psychotic symptoms and treatment resistant schizophrenia: European journal of pharmacology, v. 682 , no. $1-3$, p. 1-11, doi: 10.1016/j.ejphar.2012.02.033.

Bayer, T.A., Falkai, P., and Maier, W., 1999, Genetic and non-genetic vulnerability factors in schizophrenia: the basis of the "two hit hypothesis:" Journal of psychiatric research, $v$. 33 , no. 6 , p. 543-548.

Becker, V., 2005, [Rokitansky and Virchow: throes about the scientific term of disease]: Wiener medizinische Wochenschrift (1946), v. 155, no. 19-20, p. 463-467, doi: 10.1007/s10354-005-0217-x.

Belforte, J.E., Zsiros, V., Sklar, E.R., Jiang, Z., Yu, G., Li, Y., Quinlan, E.M., and Nakazawa, K., 2010, Postnatal NMDA receptor ablation in corticolimbic interneurons confers schizophrenia-like phenotypes: Nature neuroscience, v. 13 , no. 1 , p. $76-83$, doi: 10.1038/nn.2447.

Berganza, C.E., Mezzich, J.E., and Pouncey, C., 2005, Concepts of disease: their relevance for psychiatric diagnosis and classification: Psychopathology, v. 38, no. 4, p. 166-170, doi: $10.1159 / 000086084$.

Bertani, G., 1951, Studies on lysogenesis. I. The mode of phage liberation by lysogenic Escherichia coli: Journal of bacteriology, v. 62, no. 3, p. 293-300.

Bian, S.-Z., Zhang, J., Liu, W.-L., Sun, Z.-H., Gu, Z.-L., and Jiang, X.-G., 2009, [Receptor antagonist of NMDA and animal models of schizophrenia]: Fa yi xue za zhi, v. 25, no. 6, p. 443-446.

Biedermann, F., and Fleischhacker, W.W., 2009, Antipsychotics in the early stage of development: Current opinion in psychiatry, v. 22, no. 3, p. 326-330, doi: 10.1097/YCO.0b013e328329cd73.

Birnboim, H.C., and Doly, J., 1979, A rapid alkaline extraction procedure for screening recombinant plasmid DNA: Nucleic acids research, v. 7, no. 6, p. 1513-1523.

Bjarnadottir, M., Misner, D.L., Haverfield-Gross, S., Bruun, S., Helgason, V.G., Stefansson, H., Sigmundsson, A., Firth, D.R., Nielsen, B., Stefansdottir, R., Novak, T.J., Stefansson, K., Gurney, M.E., and Andresson, T., 2007, Neuregulin1 (NRG1) signaling through Fyn modulates NMDA receptor phosphorylation: differential synaptic function in NRG1+/- knock-outs compared with wild-type mice: The Journal of neuroscience: the official journal of the Society for Neuroscience, v. 27 , no. 17, p. 4519-4529, doi: 10.1523/JNEUROSCI.4314-06.2007.

Borasio, G.D., 1990, Differential effects of the protein kinase inhibitor K-252a on the in vitro survival of chick embryonic neurons: Neuroscience letters, v. 108, no. 1-2, p. 207212. 
Botvinnik, A., Wichert, S.P., Fischer, T.M., and Rossner, M.J., 2010, Integrated analysis of receptor activation and downstream signaling with EXTassays: Nature methods, v. 7, no. 1 , p. $74-80$, doi: $10.1038 /$ nmeth. 1407 .

Boute, N., Jockers, R., and Issad, T., 2002, The use of resonance energy transfer in highthroughput screening: BRET versus FRET: Trends in pharmacological sciences, v. 23, no. 8, p. 351-354.

Braun, I., Genius, J., Grunze, H., Bender, A., Möller, H.-J., and Rujescu, D., 2007, Alterations of hippocampal and prefrontal GABAergic interneurons in an animal model of psychosis induced by NMDA receptor antagonism: Schizophrenia research, v. 97, no. 1-3, p. 254-263, doi: 10.1016/j.schres.2007.05.005.

Brennand, K.J., and Gage, F.H., 2011, Concise review: the promise of human induced pluripotent stem cell-based studies of schizophrenia: Stem cells (Dayton, Ohio), v. 29, no. 12 , p. 1915-1922, doi: 10.1002/stem.762.

Brennand, K.J., and Gage, F.H., 2012, Modeling psychiatric disorders through reprogramming: Disease models \& mechanisms, v. 5 , no. 1, p. 26-32, doi: $10.1242 / \mathrm{dmm} .008268$.

Brennand, K.J., Simone, A., Jou, J., Gelboin-Burkhart, C., Tran, N., Sangar, S., Li, Y., Mu, Y., Chen, G., Yu, D., McCarthy, S., Sebat, J., and Gage, F.H., 2011, Modelling schizophrenia using human induced pluripotent stem cells: Nature, v. 473, no. 7346, p. 221-225, doi: 10.1038/nature09915.

Brewster, U.C., and Perazella, M.A., 2004, The renin-angiotensin-aldosterone system and the kidney: effects on kidney disease: The American journal of medicine, v. 116, no. 4, p. 263-272, doi: 10.1016/j.amjmed.2003.09.034.

Brinkmann, B.G., Agarwal, A., Sereda, M.W., Garratt, A.N., Müller, T., Wende, H., Stassart, R.M., Nawaz, S., Humml, C., Velanac, V., Radyushkin, K., Goebbels, S., Fischer, T.M., Franklin, R.J., et al., 2008, Neuregulin-1/ErbB signaling serves distinct functions in myelination of the peripheral and central nervous system: Neuron, v. 59, no. 4, p. 581-595, doi: 10.1016/j.neuron.2008.06.028.

Brockes, J.P., Lemke, G.E., and Balzer, D.R., Jr, 1980, Purification and preliminary characterization of a glial growth factor from the bovine pituitary: The Journal of biological chemistry, v. 255 , no. 18 , p. $8374-8377$.

Buonanno, A., 2010, The neuregulin signaling pathway and schizophrenia: from genes to synapses and neural circuits: Brain research bulletin, v. 83, no. 3-4, p. 122-131, doi: 10.1016/j.brainresbull.2010.07.012.

Burgess, A.W., Cho, H.-S., Eigenbrot, C., Ferguson, K.M., Garrett, T.P.J., Leahy, D.J., Lemmon, M.A., Sliwkowski, M.X., Ward, C.W., and Yokoyama, S., 2003, An openand-shut case? Recent insights into the activation of EGF/ErbB receptors: Molecular cell, v. 12 , no. 3, p. 541-552.

Cantor-Graae, E., and Selten, J.-P., 2005, Schizophrenia and migration: a meta-analysis and review: The American journal of psychiatry, v. 162, no. 1, p. 12-24, doi: 10.1176/appi.ajp.162.1.12. 
Capdevila-Nortes, X., López-Hernández, T., Ciruela, F., and Estévez, R., 2012, A modification of the split-tobacco etch virus method for monitoring interactions between membrane proteins in mammalian cells: Analytical biochemistry, v. 423 , no. 1, p. 109-118, doi: 10.1016/j.ab.2012.01.022.

Carlén, M., Meletis, K., Siegle, J.H., Cardin, J.A., Futai, K., Vierling-Claassen, D., Rühlmann, C., Jones, S.R., Deisseroth, K., Sheng, M., Moore, C.I., and Tsai, L.-H., 2012, A critical role for NMDA receptors in parvalbumin interneurons for gamma rhythm induction and behavior: Molecular psychiatry, v. 17 , no. 5, p. 537-548, doi: 10.1038/mp.2011.31.

Carlsson, A., Waters, N., and Carlsson, M.L., 1999, Neurotransmitter interactions in schizophrenia-therapeutic implications: European archives of psychiatry and clinical neuroscience, v. 249 Suppl 4, p. 37-43.

Carol, H., Houghton, P.J., Morton, C.L., Kolb, E.A., Gorlick, R., Reynolds, C.P., Kang, M.H., Maris, J.M., Keir, S.T., Watkins, A., Smith, M.A., and Lock, R.B., 2010, Initial Testing of Topotecan by the Pediatric Preclinical Testing Program: Pediatric blood \& cancer, v. 54 , no. 5 , p. $707-715$, doi: $10.1002 /$ pbc. 22352 .

Carpenter, G., 2003, ErbB-4: mechanism of action and biology: Experimental cell research, v. 284 , no. 1 , p. $66-77$.

Carraway, K.L., 3rd, Sliwkowski, M.X., Akita, R., Platko, J.V., Guy, P.M., Nuijens, A., Diamonti, A.J., Vandlen, R.L., Cantley, L.C., and Cerione, R.A., 1994, The erbB3 gene product is a receptor for heregulin: The Journal of biological chemistry, v. 269, no. 19, p. 14303-14306.

Castoldi, R., Jucknischke, U., Pradel, L.P., Arnold, E., Klein, C., Scheiblich, S., Niederfellner, G., and Sustmann, C., 2012, Molecular characterization of novel trispecific ErbBcMet-IGF1R antibodies and their antigen-binding properties: Protein Engineering Design and Selection,, doi: 10.1093/protein/gzs048.

Chen, Y.-J.J., Johnson, M.A., Lieberman, M.D., Goodchild, R.E., Schobel, S., Lewandowski, N., Rosoklija, G., Liu, R.-C., Gingrich, J.A., Small, S., Moore, H., Dwork, A.J., Talmage, D.A., and Role, L.W., 2008, Type III neuregulin-1 is required for normal sensorimotor gating, memory-related behaviors, and corticostriatal circuit components: The Journal of neuroscience: the official journal of the Society for Neuroscience, v. 28, no. 27, p. 6872-6883, doi: 10.1523/JNEUROSCI.1815-08.2008.

Chen, S.-K., Tvrdik, P., Peden, E., Cho, S., Wu, S., Spangrude, G., and Capecchi, M.R., 2010, Hematopoietic origin of pathological grooming in Hoxb8 mutant mice: Cell, v. 141 , no. 5, p. 775-785, doi: 10.1016/j.cell.2010.03.055.

Cheng, K.C.-C., and Inglese, J., 2012, A coincidence reporter-gene system for highthroughput screening: Nature methods, v. 9, no. 10, p. 937, doi: 10.1038/nmeth.2170.

Clower, W.T., and Finger, S., 2001, Discovering trepanation: the contribution of Paul Broca: Neurosurgery, v. 49, no. 6, p. 1417-1425; discussion 1425-1426.

Davis, K.L., Kahn, R.S., Ko, G., and Davidson, M., 1991, Dopamine in schizophrenia: a review and reconceptualization: The American journal of psychiatry, v. 148, no. 11, p. 1474-1486. 
Desbonnet, L., Waddington, J.L., and O'Tuathaigh, C.M.P., 2009, Mutant models for genes associated with schizophrenia: Biochemical Society transactions, v. 37, no. Pt 1, p. 308-312, doi: 10.1042/BST0370308.

Djannatian, M.S., Galinski, S., Fischer, T.M., and Rossner, M.J., 2011, Studying G proteincoupled receptor activation using split-tobacco etch virus assays: Analytical biochemistry, v. 412, no. 2, p. 141-152, doi: 10.1016/j.ab.2011.01.042.

Eaton, W.W., Hall, A.L.F., Macdonald, R., and McKibben, J., 2007, Case identification in psychiatric epidemiology: a review: International review of psychiatry (Abingdon, England), v. 19, no. 5, p. 497-507, doi: 10.1080/09540260701564906.

Eitner, K., and Koch, U., 2009, From fragment screening to potent binders: strategies for fragment-to-lead evolution: Mini reviews in medicinal chemistry, v. 9, no. 8, p. 956961.

Elenius, K., Choi, C.J., Paul, S., Santiestevan, E., Nishi, E., and Klagsbrun, M., 1999, Characterization of a naturally occurring ErbB4 isoform that does not bind or activate phosphatidyl inositol 3-kinase: Oncogene, v. 18, no. 16, p. 2607-2615, doi: 10.1038/sj.onc.1202612.

Ellard, J., 1987, Did schizophrenia exist before the eighteenth century?: The Australian and New Zealand journal of psychiatry, v. 21, no. 3, p. 306-318.

Emamian, E.S., 2012, AKT/GSK3 signaling pathway and schizophrenia: Frontiers in molecular neuroscience, v. 5, p. 33, doi: 10.3389/fnmol.2012.00033.

Eyjolfsson, E.M., Brenner, E., Kondziella, D., and Sonnewald, U., 2006, Repeated injection of MK801: an animal model of schizophrenia?: Neurochemistry international, v. 48, no. 6-7, p. 541-546, doi: 10.1016/j.neuint.2005.11.019.

Fagart, J., Hillisch, A., Huyet, J., Bärfacker, L., Fay, M., Pleiss, U., Pook, E., Schäfer, S., Rafestin-Oblin, M.-E., and Kolkhof, P., 2010, A new mode of mineralocorticoid receptor antagonism by a potent and selective nonsteroidal molecule: The Journal of biological chemistry, v. 285, no. 39, p. 29932-29940, doi: 10.1074/jbc.M110.131342.

Falls, D.L., 2003, Neuregulins: functions, forms, and signaling strategies: Experimental cell research, v. 284 , no. 1 , p. 14-30.

Fazzari, P., Paternain, A.V., Valiente, M., Pla, R., Luján, R., Lloyd, K., Lerma, J., Marín, O., and Rico, B., 2010, Control of cortical GABA circuitry development by $\mathrm{Nrg} 1$ and ErbB4 signalling: Nature, v. 464, no. 7293 , p. 1376-1380, doi: 10.1038/nature08928.

Ferentinos, P., and Dikeos, D., 2012, Genetic correlates of medical comorbidity associated with schizophrenia and treatment with antipsychotics: Current opinion in psychiatry, v. 25, no. 5, p. 381-390, doi: 10.1097/YCO.0b013e3283568537.

Di Fiore, P.P., Segatto, O., Lonardo, F., Fazioli, F., Pierce, J.H., and Aaronson, S.A., 1990, The carboxy-terminal domains of erbB-2 and epidermal growth factor receptor exert different regulatory effects on intrinsic receptor tyrosine kinase function and transforming activity: Molecular and cellular biology, v. 10, no. 6, p. 2749-2756. 
Fisahn, A., Neddens, J., Yan, L., and Buonanno, A., 2009, Neuregulin-1 modulates hippocampal gamma oscillations: implications for schizophrenia: Cerebral cortex (New York, N.Y.: 1991), v. 19, no. 3, p. 612-618, doi: 10.1093/cercor/bhn107.

Fishman, M.C., and Porter, J.A., 2005, Pharmaceuticals: a new grammar for drug discovery: Nature, v. 437, no. 7058 , p. 491-493, doi: 10.1038/437491a.

Flames, N., Long, J.E., Garratt, A.N., Fischer, T.M., Gassmann, M., Birchmeier, C., Lai, C., Rubenstein, J.L.R., and Marín, O., 2004, Short- and long-range attraction of cortical GABAergic interneurons by neuregulin-1: Neuron, v. 44, no. 2, p. 251-261, doi: 10.1016/j.neuron.2004.09.028.

Fleck, D., Garratt, A.N., Haass, C., and Willem, M., 2011, BACE1 Dependent Neuregulin Proteolysis: Current Alzheimer research,

Flores, C., and Coyle, J.T., 2003, Regulation of glutamate carboxypeptidase II function in corticolimbic regions of rat brain by phencyclidine, haloperidol, and clozapine: Neuropsychopharmacology: official publication of the American College of Neuropsychopharmacology, v. 28, no. 7, p. 1227-1234, doi: 10.1038/sj.npp.1300129.

Fornito, A., Zalesky, A., Pantelis, C., and Bullmore, E.T., 2012, Schizophrenia, neuroimaging and connectomics: Neurolmage, v. 62, no. 4, p. 2296-2314, doi: 10.1016/j.neuroimage.2011.12.090.

Freyer, M.W., and Lewis, E.A., 2008, Isothermal titration calorimetry: experimental design, data analysis, and probing macromolecule/ligand binding and kinetic interactions: Methods in cell biology, v. 84, p. 79-113, doi: 10.1016/S0091-679X(07)84004-0.

Fried, K., Risling, M., Tidcombe, H., Gassmann, M., and Lillesaar, C., 2002, Expression of ErbB3, ErbB4, and neuregulin-1 mRNA during tooth development: Developmental dynamics: an official publication of the American Association of Anatomists, v. 224, no. 3, p. 356-360, doi: 10.1002/dvdy.10114.

Fuller, P.J., Yao, Y., Yang, J., and Young, M.J., 2012, Mechanisms of ligand specificity of the mineralocorticoid receptor: The Journal of endocrinology, v. 213, no. 1, p. 15-24, doi: 10.1530/JOE-11-0372.

Gao, Z., and Jacobson, K.A., 2002, 2-Chloro-N(6)-cyclopentyladenosine, adenosine A(1) receptor agonist, antagonizes the adenosine $A(3)$ receptor: European journal of pharmacology, v. 443, no. 1-3, p. 39-42.

Gaunitz, F., and Papke, M., 1998, Gene transfer and expression: Methods in molecular biology (Clifton, N.J.), v. 107, p. 361-370, doi: 10.1385/0-89603-519-0:361.

Geerling, J.C., and Loewy, A.D., 2009, Aldosterone in the brain: American journal of physiology. Renal physiology, v. 297, no. 3, p. F559-576, doi: 10.1152/ajprenal.90399.2008.

Goff, D.C., and Coyle, J.T., 2001, The emerging role of glutamate in the pathophysiology and treatment of schizophrenia: The American journal of psychiatry, v. 158, no. 9, p. 1367-1377.

Goldman-Rakic, P.S., Castner, S.A., Svensson, T.H., Siever, L.J., and Williams, G.V., 2004, Targeting the dopamine D1 receptor in schizophrenia: insights for cognitive 
dysfunction: Psychopharmacology, v. 174 , no. 1, p. 3-16, doi: 10.1007/s00213-0041793-y.

Greene, L.A., and Tischler, A.S., 1976, Establishment of a noradrenergic clonal line of rat adrenal pheochromocytoma cells which respond to nerve growth factor: Proceedings of the National Academy of Sciences of the United States of America, v. 73, no. 7, p. 2424-2428.

Gromova, I.I., Kjeldsen, E., Svejstrup, J.Q., Alsner, J., Christiansen, K., and Westergaard, O., 1993, Characterization of an altered DNA catalysis of a camptothecin-resistant eukaryotic topoisomerase I: Nucleic acids research, v. 21, no. 3, p. 593-600.

Grossmann, C., Ruhs, S., Langenbruch, L., Mildenberger, S., Strätz, N., Schumann, K., and Gekle, M., 2012, Nuclear shuttling precedes dimerization in mineralocorticoid receptor signaling: Chemistry \& biology, v. 19, no. 6, p. 742-751, doi: 10.1016/j.chembiol.2012.04.014.

Grossmann, K.S., Wende, H., Paul, F.E., Cheret, C., Garratt, A.N., Zurborg, S., Feinberg, K., Besser, D., Schulz, H., Peles, E., Selbach, M., Birchmeier, W., and Birchmeier, C., 2009, The tyrosine phosphatase Shp2 (PTPN11) directs Neuregulin-1/ErbB signaling throughout Schwann cell development: Proceedings of the National Academy of Sciences of the United States of America, v. 106, no. 39, p. 16704-16709, doi: 10.1073/pnas.0904336106.

Grunze, H.C., Rainnie, D.G., Hasselmo, M.E., Barkai, E., Hearn, E.F., McCarley, R.W., and Greene, R.W., 1996, NMDA-dependent modulation of CA1 local circuit inhibition: The Journal of neuroscience: the official journal of the Society for Neuroscience, v. 16, no. 6, p. 2034-2043.

Gunduz-Bruce, H., 2009, The acute effects of NMDA antagonism: from the rodent to the human brain: Brain research reviews, v. 60 , no. 2, p. 279-286, doi: 10.1016/j.brainresrev.2008.07.006.

Haenschel, C., Bittner, R.A., Waltz, J., Haertling, F., Wibral, M., Singer, W., Linden, D.E.J., and Rodriguez, E., 2009, Cortical oscillatory activity is critical for working memory as revealed by deficits in early-onset schizophrenia: The Journal of neuroscience: the official journal of the Society for Neuroscience, v. 29 , no. 30, p. 9481-9489, doi: 10.1523/JNEUROSCI.1428-09.2009.

Hall, J., Whalley, H.C., Job, D.E., Baig, B.J., McIntosh, A.M., Evans, K.L., Thomson, P.A., Porteous, D.J., Cunningham-Owens, D.G., Johnstone, E.C., and Lawrie, S.M., 2006, A neuregulin 1 variant associated with abnormal cortical function and psychotic symptoms: Nature neuroscience, v. 9, no. 12, p. 1477-1478, doi: 10.1038/nn1795.

Hancock, M.L., Canetta, S.E., Role, L.W., and Talmage, D.A., 2008, Presynaptic type III neuregulin1-ErbB signaling targets alpha7 nicotinic acetylcholine receptors to axons: The Journal of general physiology, v. 131, no. 6, p. i4, doi: 10.1085/JGP1316OIA4.

Hanisch, U.-K., and Kettenmann, H., 2007, Microglia: active sensor and versatile effector cells in the normal and pathologic brain: Nature neuroscience, v. 10, no. 11, p. 13871394, doi: $10.1038 / \mathrm{nn} 1997$. 
Harrison, P.J., and Weinberger, D.R., 2005, Schizophrenia genes, gene expression, and neuropathology: on the matter of their convergence: Molecular psychiatry, v. 10, no. 1 , p. 40-68; image 5, doi: 10.1038/sj.mp.4001558.

Hartley, J.L., Temple, G.F., and Brasch, M.A., 2000, DNA cloning using in vitro site-specific recombination: Genome research, v. 10, no. 11, p. 1788-1795.

Hashimoto, T., Volk, D.W., Eggan, S.M., Mirnics, K., Pierri, J.N., Sun, Z., Sampson, A.R., and Lewis, D.A., 2003, Gene expression deficits in a subclass of GABA neurons in the prefrontal cortex of subjects with schizophrenia: The Journal of neuroscience: the official journal of the Society for Neuroscience, v. 23, no. 15, p. 6315-6326.

Höistad, M., Segal, D., Takahashi, N., Sakurai, T., Buxbaum, J.D., and Hof, P.R., 2009, Linking white and grey matter in schizophrenia: oligodendrocyte and neuron pathology in the prefrontal cortex: Frontiers in neuroanatomy, v. 3, p. 9, doi: 10.3389/neuro.05.009.2009.

Holdgate, G.A., Anderson, M., Edfeldt, F., and Geschwindner, S., 2010, Affinity-based, biophysical methods to detect and analyze ligand binding to recombinant proteins: matching high information content with high throughput: Journal of structural biology, v. 172 , no. 1, p. 142-157, doi: 10.1016/j.jsb.2010.06.024.

Holsboer, F., 1999, The rationale for corticotropin-releasing hormone receptor (CRH-R) antagonists to treat depression and anxiety: Journal of psychiatric research, v. 33, no. 3, p. 181-214.

Homayoun, H., and Moghaddam, B., 2007, NMDA Receptor Hypofunction Produces Opposite Effects on Prefrontal Cortex Interneurons and Pyramidal Neurons: The Journal of Neuroscience, v. 27 , no. 43 , p. 11496-11500, doi: 10.1523/JNEUROSCI.221307.2007.

Honea, R.A., Meyer-Lindenberg, A., Hobbs, K.B., Pezawas, L., Mattay, V.S., Egan, M.F., Verchinski, B., Passingham, R.E., Weinberger, D.R., and Callicott, J.H., 2008, Is gray matter volume an intermediate phenotype for schizophrenia? A voxel-based morphometry study of patients with schizophrenia and their healthy siblings: Biological psychiatry, v. 63, no. 5, p. 465-474, doi: 10.1016/j.biopsych.2007.05.027.

Hong, L.E., Wonodi, I., Stine, O.C., Mitchell, B.D., and Thaker, G.K., 2008, Evidence of missense mutations on the neuregulin 1 gene affecting function of prepulse inhibition: Biological psychiatry, v. 63, no. 1, p. 17-23, doi: 10.1016/j.biopsych.2007.05.011.

Houghton, P.J., Cheshire, P.J., Hallman, J.D., 2nd, Lutz, L., Friedman, H.S., Danks, M.K., and Houghton, J.A., 1995, Efficacy of topoisomerase I inhibitors, topotecan and irinotecan, administered at low dose levels in protracted schedules to mice bearing xenografts of human tumors: Cancer chemotherapy and pharmacology, v. 36 , no. 5, p. 393-403.

Hsiang, Y.H., Liu, L.F., Wall, M.E., Wani, M.C., Nicholas, A.W., Manikumar, G., Kirschenbaum, S., Silber, R., and Potmesil, M., 1989, DNA topoisomerase I-mediated DNA cleavage and cytotoxicity of camptothecin analogues: Cancer research, v. 49, no. 16, p. $4385-4389$. 
Huber, W., 2005, A new strategy for improved secondary screening and lead optimization using high-resolution SPR characterization of compound-target interactions: Journal of molecular recognition: JMR, v. 18, no. 4, p. 273-281, doi: 10.1002/jmr.744.

Hudepohl, N.S., and Nasrallah, H.A., 2012, Antipsychotic drugs: Handbook of clinical neurology / edited by P.J. Vinken and G.W. Bruyn, v. 106, p. 657-667, doi: 10.1016/B978-0-444-52002-9.00039-5.

Hudis, C.A., 2007, Trastuzumab--mechanism of action and use in clinical practice: The New England journal of medicine, v. 357, no. 1, p. 39-51, doi: 10.1056/NEJMra043186.

Inglese, J., Johnson, R.L., Simeonov, A., Xia, M., Zheng, W., Austin, C.P., and Auld, D.S., 2007, High-throughput screening assays for the identification of chemical probes: Nature chemical biology, v. 3, no. 8, p. 466-479, doi: 10.1038/nchembio.2007.17.

Ishii, R., Takahashi, H., Kurimoto, R., Aoki, Y., Ikeda, S., Hata, M., Ikezawa, K., Canuet, L., Nakahachi, T., Iwase, M., and Takeda, M., 2012, [Endophenotypes in schizophrenia: a review of electrophysiological studies]: Seishin shinkeigaku zasshi $=$ Psychiatria et neurologia Japonica, v. 114, no. 6, p. 629-646.

Jaaro-Peled, H., Hayashi-Takagi, A., Seshadri, S., Kamiya, A., Brandon, N.J., and Sawa, A., 2009, Neurodevelopmental mechanisms of schizophrenia: understanding disturbed postnatal brain maturation through neuregulin-1-ErbB4 and DISC1: Trends in neurosciences, v. 32, no. 9, p. 485-495, doi: 10.1016/j.tins.2009.05.007.

Javitt, D.C., and Zukin, S.R., 1991, Recent advances in the phencyclidine model of schizophrenia: The American journal of psychiatry, v. 148, no. 10, p. 1301-1308.

Jeste, D.V., del Carmen, R., Lohr, J.B., and Wyatt, R.J., 1985, Did schizophrenia exist before the eighteenth century?: Comprehensive psychiatry, v. 26, no. 6, p. 493-503.

Jia, P., Sun, J., Guo, A.Y., and Zhao, Z., 2010, SZGR: a comprehensive schizophrenia gene resource: Molecular psychiatry, v. 15, no. 5, p. 453-462, doi: 10.1038/mp.2009.93.

Jones, R.B., Gordus, A., Krall, J.A., and MacBeath, G., 2006, A quantitative protein interaction network for the ErbB receptors using protein microarrays: Nature, v. 439, no. 7073 , p. 168-174, doi: 10.1038/nature04177.

Jordan, M.A., Himes, R.H., and Wilson, L., 1985, Comparison of the effects of vinblastine, vincristine, vindesine, and vinepidine on microtubule dynamics and cell proliferation in vitro: Cancer research, v. 45 , no. 6 , p. 2741-2747.

Kang, E., Burdick, K.E., Kim, J.Y., Duan, X., Guo, J.U., Sailor, K.A., Jung, D.-E., Ganesan, S., Choi, S., Pradhan, D., Lu, B., Avramopoulos, D., Christian, K., Malhotra, A.K., et al., 2011, Interaction between FEZ1 and DISC1 in regulation of neuronal development and risk for schizophrenia: Neuron, v. 72, no. 4, p. 559-571, doi: 10.1016/j.neuron.2011.09.032.

Karam, C.S., Ballon, J.S., Bivens, N.M., Freyberg, Z., Girgis, R.R., Lizardi-Ortiz, J.E., Markx, S., Lieberman, J.A., and Javitch, J.A., 2010, Signaling pathways in schizophrenia: emerging targets and therapeutic strategies: Trends in pharmacological sciences, v. 31, no. 8, p. 381-390, doi: 10.1016/j.tips.2010.05.004. 
Karaman, M.W., Herrgard, S., Treiber, D.K., Gallant, P., Atteridge, C.E., Campbell, B.T., Chan, K.W., Ciceri, P., Davis, M.I., Edeen, P.T., Faraoni, R., Floyd, M., Hunt, J.P., Lockhart, D.J., et al., 2008, A quantitative analysis of kinase inhibitor selectivity: Nature biotechnology, v. 26, no. 1, p. 127-132, doi: 10.1038/nbt1358.

Kato, T., Kasai, A., Mizuno, M., Fengyi, L., Shintani, N., Maeda, S., Yokoyama, M., Ozaki, M., and Nawa, H., 2010, Phenotypic characterization of transgenic mice overexpressing neuregulin-1: PloS one, v. 5, no. 12, p. e14185, doi: 10.1371/journal.pone.0014185.

Kawato, Y., Aonuma, M., Hirota, Y., Kuga, H., and Sato, K., 1991, Intracellular roles of SN-38, a metabolite of the camptothecin derivative CPT-11, in the antitumor effect of CPT11: Cancer research, v. 51, no. 16, p. 4187-4191.

Keely, S.J., Calandrella, S.O., and Barrett, K.E., 2000, Carbachol-stimulated transactivation of epidermal growth factor receptor and mitogen-activated protein kinase in $T(84)$ cells is mediated by intracellular ca(2+), PYK-2, and p60(src): The Journal of biological chemistry, v. 275 , no. 17 , p. 12619-12625.

Kellendonk, C., Simpson, E.H., and Kandel, E.R., 2009, Modeling cognitive endophenotypes of schizophrenia in mice: Trends in neurosciences, v. 32, no. 6, p. 347-358, doi: 10.1016/j.tins.2009.02.003.

Keshavan, M.S., Tandon, R., Boutros, N.N., and Nasrallah, H.A., 2008, Schizophrenia, "just the facts": what we know in 2008 Part 3: neurobiology: Schizophrenia research, v. 106, no. 2-3, p. 89-107, doi: 10.1016/j.schres.2008.07.020.

Kibbe, W.A., 2007, OligoCalc: an online oligonucleotide properties calculator: Nucleic acids research, v. 35, no. Web Server issue, p. W43-46, doi: 10.1093/nar/gkm234.

Kirkbride, J.B., Fearon, P., Morgan, C., Dazzan, P., Morgan, K., Tarrant, J., Lloyd, T., Holloway, J., Hutchinson, G., Leff, J.P., Mallett, R.M., Harrison, G.L., Murray, R.M., and Jones, P.B., 2006, Heterogeneity in incidence rates of schizophrenia and other psychotic syndromes: findings from the 3-center AeSOP study: Archives of general psychiatry, v. 63 , no. 3, p. 250-258, doi: 10.1001/archpsyc.63.3.250.

Kisseleva, T., Bhattacharya, S., Braunstein, J., and Schindler, C.W., 2002, Signaling through the JAK/STAT pathway, recent advances and future challenges: Gene, v. 285, no. 1 2, p. 1-24.

Kleinman, J.E., Law, A.J., Lipska, B.K., Hyde, T.M., Ellis, J.K., Harrison, P.J., and Weinberger, D.R., 2011, Genetic neuropathology of schizophrenia: new approaches to an old question and new uses for postmortem human brains: Biological psychiatry, v. 69, no. 2, p. 140-145, doi: 10.1016/j.biopsych.2010.10.032.

Klosterkötter, J., 2008, Indicated prevention of schizophrenia: Deutsches Ärzteblatt international, v. 105, no. 30, p. 532-539, doi: 10.3238/arztebl.2008.0532.

Kocisko, D.A., Baron, G.S., Rubenstein, R., Chen, J., Kuizon, S., and Caughey, B., 2003, New inhibitors of scrapie-associated prion protein formation in a library of 2000 drugs and natural products: Journal of virology, v. 77 , no. 19, p. 10288-10294.

Kodesh, A., Goldshtein, I., Gelkopf, M., Goren, I., Chodick, G., and Shalev, V., 2012, Epidemiology and comorbidity of severe mental illnesses in the community: findings 
from a computerized mental health registry in a large Israeli health organization: Social psychiatry and psychiatric epidemiology,, doi: 10.1007/s00127-012-0478-9.

Konradi, C., Yang, C.K., Zimmerman, E.I., Lohmann, K.M., Gresch, P., Pantazopoulos, H., Berretta, S., and Heckers, S., 2011, Hippocampal interneurons are abnormal in schizophrenia: Schizophrenia research, v. 131, no. 1-3, p. 165-173, doi: 10.1016/j.schres.2011.06.007.

Kraepelin, E. 1896: Psychiatrie: Ein Lehrbuch für Studierende und Ärzte. Fünfte, vollständig umgearbeitete Auflage. Leipzig.

Kuai, L., Ong, S.-E., Madison, J.M., Wang, X., Duvall, J.R., Lewis, T.A., Luce, C.J., Conner, S.D., Pearlman, D.A., Wood, J.L., Schreiber, S.L., Carr, S.A., Scolnick, E.M., and Haggarty, S.J., 2011, AAK1 identified as an inhibitor of neuregulin-1/ErbB4dependent neurotrophic factor signaling using integrative chemical genomics and proteomics: Chemistry \& biology, v. 18 , no. 7 , p. 891-906, doi: 10.1016/j.chembiol.2011.03.017.

Kuai, L., Wang, X., Madison, J.M., Schreiber, S.L., Scolnick, E.M., and Haggarty, S.J., 2010, Chemical genetics identifies small-molecule modulators of neuritogenesis involving neuregulin-1/ErbB4 signaling: ACS chemical neuroscience, v. 1, no. 4, p. 325-342, doi: $10.1021 / \mathrm{cn} 900046 \mathrm{a}$.

Kuhn, J.G., 1998, Pharmacology of irinotecan: Oncology (Williston Park, N.Y.), v. 12, no. 8 Suppl 6, p. 39-42.

Kwon, O.B., Paredes, D., Gonzalez, C.M., Neddens, J., Hernandez, L., Vullhorst, D., and Buonanno, A., 2008, Neuregulin-1 regulates LTP at CA1 hippocampal synapses through activation of dopamine D4 receptors: Proceedings of the National Academy of Sciences of the United States of America, v. 105, no. 40, p. 15587-15592, doi: 10.1073/pnas.0805722105.

Law, A.J., Kleinman, J.E., Weinberger, D.R., and Weickert, C.S., 2007, Disease-associated intronic variants in the ErbB4 gene are related to altered ErbB4 splice-variant expression in the brain in schizophrenia: Human molecular genetics, v. 16, no. 2, p. 129-141, doi: $10.1093 / \mathrm{hmg} / \mathrm{ddl} 449$.

Law, A.J., Wang, Y., Sei, Y., O'Donnell, P., Piantadosi, P., Papaleo, F., Straub, R.E., Huang, W., Thomas, C.J., Vakkalanka, R., Besterman, A.D., Lipska, B.K., Hyde, T.M., Harrison, P.J., et al., 2012b, Neuregulin 1-ErbB4-PI3K signaling in schizophrenia and phosphoinositide 3-kinase-p110ठ inhibition as a potential therapeutic strategy: Proceedings of the National Academy of Sciences of the United States of America, doi: 10.1073/pnas.1206118109.

Leevers, S.J., Vanhaesebroeck, B., and Waterfield, M.D., 1999, Signalling through phosphoinositide 3-kinases: the lipids take centre stage: Current opinion in cell biology, v. 11, no. 2, p. 219-225.

Lewis, D.A., and González-Burgos, G., 2008, Neuroplasticity of neocortical circuits in schizophrenia: Neuropsychopharmacology: official publication of the American College of Neuropsychopharmacology, v. 33, no. 1, p. 141-165, doi: 10.1038/sj.npp.1301563. 
Lewis, D.A., Hashimoto, T., and Volk, D.W., 2005, Cortical inhibitory neurons and schizophrenia: Nature reviews. Neuroscience, v. 6, no. 4, p. 312-324, doi: $10.1038 /$ nrn1648.

Lewis, D.A., and Levitt, P., 2002, Schizophrenia as a disorder of neurodevelopment: Annual review of neuroscience, v. 25, p. 409-432, doi: 10.1146/annurev.neuro.25.112701.142754.

Lewis, D.A., and Lieberman, J.A., 2000, Catching up on schizophrenia: natural history and neurobiology: Neuron, v. 28, no. 2, p. 325-334.

Li, D., Collier, D.A., and He, L., 2006, Meta-analysis shows strong positive association of the neuregulin 1 (NRG1) gene with schizophrenia: Human molecular genetics, v. 15, no. 12, p. 1995-2002, doi: $10.1093 / \mathrm{hmg} / \mathrm{ddl} 122$.

Li, T., Stefansson, H., Gudfinnsson, E., Cai, G., Liu, X., Murray, R.M., Steinthorsdottir, V., Januel, D., Gudnadottir, V.G., Petursson, H., Ingason, A., Gulcher, J.R., Stefansson, K., and Collier, D.A., 2004, Identification of a novel neuregulin 1 at-risk haplotype in Han schizophrenia Chinese patients, but no association with the Icelandic/Scottish risk haplotype: Molecular psychiatry, v. 9, no. 7, p. 698-704, doi: 10.1038/sj.mp.4001485.

Lievens, S., Caligiuri, M., Kley, N., and Tavernier, J., 2012, The use of mammalian two-hybrid technologies for high-throughput drug screening: Methods (San Diego, Calif.),, doi: 10.1016/j.ymeth.2012.08.003.

Liu, P., Cleveland, T.E., 4th, Bouyain, S., Byrne, P.O., Longo, P.A., and Leahy, D.J., 2012, A single ligand is sufficient to activate EGFR dimers: Proceedings of the National Academy of Sciences of the United States of America, v. 109, no. 27, p. 1086110866, doi: 10.1073/pnas.1201114109.

Liu, X., Robinson, G.W., Wagner, K.U., Garrett, L., Wynshaw-Boris, A., and Hennighausen, L., 1997, Stat5a is mandatory for adult mammary gland development and lactogenesis: Genes \& development, v. 11, no. 2, p. 179-186.

Lodge, D., and Anis, N.A., 1982, Effects of phencyclidine on excitatory amino acid activation of spinal interneurones in the cat: European journal of pharmacology, v. 77, no. 2-3, p. 203-204.

Lonardo, F., Di Marco, E., King, C.R., Pierce, J.H., Segatto, O., Aaronson, S.A., and Di Fiore, P.P., 1990, The normal erbB-2 product is an atypical receptor-like tyrosine kinase with constitutive activity in the absence of ligand: The New biologist, v. 2, no. 11, p. 992-1003.

Lu, C.-L., Wang, Y.-C., Chen, J.-Y., Lai, I.-C., and Liou, Y.-J., 2010, Support for the involvement of the ERBB4 gene in schizophrenia: a genetic association analysis: Neuroscience letters, v. 481, no. 2, p. 120-125, doi: 10.1016/j.neulet.2010.06.067.

LUBY, E.D., COHEN, B.D., ROSENBAUM, G., GOTTLIEB, J.S., and KELLEY, R., 1959, Study of a new schizophrenomimetic drug; sernyl: A.M.A. archives of neurology and psychiatry, v. 81 , no. 3, p. 363-369.

Luo, X., Prior, M., He, W., Hu, X., Tang, X., Shen, W., Yadav, S., Kiryu-Seo, S., Miller, R., Trapp, B.D., and Yan, R., 2011, Cleavage of neuregulin-1 by BACE1 or ADAM10 
protein produces differential effects on myelination: The Journal of biological chemistry, v. 286, no. 27, p. 23967-23974, doi: 10.1074/jbc.M111.251538.

Macarron, R., Banks, M.N., Bojanic, D., Burns, D.J., Cirovic, D.A., Garyantes, T., Green, D.V.S., Hertzberg, R.P., Janzen, W.P., Paslay, J.W., Schopfer, U., and Sittampalam, G.S., 2011, Impact of high-throughput screening in biomedical research: Nature reviews. Drug discovery, v. 10, no. 3, p. 188-195, doi: 10.1038/nrd3368.

Man, H.-Y., Wang, Q., Lu, W.-Y., Ju, W., Ahmadian, G., Liu, L., D'Souza, S., Wong, T.P., Taghibiglou, C., Lu, J., Becker, L.E., Pei, L., Liu, F., Wymann, M.P., et al., 2003, Activation of PI3-kinase is required for AMPA receptor insertion during LTP of mEPSCs in cultured hippocampal neurons: Neuron, v. 38, no. 4, p. 611-624.

Maniatis, T., Fritsch, E.F., Sambrook, J., 1982, Molecular cloning : a laboratory manual, Cold Spring Harbour Laboratory

Marín, O., 2012, Interneuron dysfunction in psychiatric disorders: Nature Reviews Neuroscience, v. 13, no. 2, p. 107-120, doi: 10.1038/nrn3155.

Marmor, M.D., Skaria, K.B., and Yarden, Y., 2004, Signal transduction and oncogenesis by ErbB/HER receptors: International journal of radiation oncology, biology, physics, v. 58, no. 3, p. 903-913, doi: 10.1016/j.ijrobp.2003.06.002.

Marzolla, V., Armani, A., Zennaro, M.-C., Cinti, F., Mammi, C., Fabbri, A., Rosano, G.M.C., and Caprio, M., 2012, The role of the mineralocorticoid receptor in adipocyte biology and fat metabolism: Molecular and cellular endocrinology, v. 350, no. 2, p. 281-288, doi: 10.1016/j.mce.2011.09.011.

Maynard, T.M., Sikich, L., Lieberman, J.A., and LaMantia, A.S., 2001, Neural development, cell-cell signaling, and the "two-hit" hypothesis of schizophrenia: Schizophrenia bulletin, v. 27 , no. 3 , p. 457-476.

McGrath, J.J., 2007, The surprisingly rich contours of schizophrenia epidemiology: Archives of general psychiatry, v. 64 , no. 1, p. 14-16, doi: 10.1001/archpsyc.64.1.14.

McIntosh, A.M., Moorhead, T.W.J., Job, D., Lymer, G.K.S., Muñoz Maniega, S., McKirdy, J., Sussmann, J.E.D., Baig, B.J., Bastin, M.E., Porteous, D., Evans, K.L., Johnstone, E.C., Lawrie, S.M., and Hall, J., 2008, The effects of a neuregulin 1 variant on white matter density and integrity: Molecular psychiatry, v. 13, no. 11, p. 1054-1059, doi: 10.1038/sj.mp.4002103.

Medina, P.J., and Goodin, S., 2008, Lapatinib: a dual inhibitor of human epidermal growth factor receptor tyrosine kinases: Clinical therapeutics, v. 30, no. 8, p. 1426-1447, doi: 10.1016/j.clinthera.2008.08.008.

Mei, L., and Xiong, W.-C., 2008, Neuregulin 1 in neural development, synaptic plasticity and schizophrenia: Nature reviews. Neuroscience, v. 9, no. 6, p. 437-452, doi: 10.1038/nrn2392.

Meyer, D., and Birchmeier, C., 1995, Multiple essential functions of neuregulin in development: Nature, v. 378, no. 6555, p. 386-390, doi: 10.1038/378386a0.

Meyer-Lindenberg, A., 2011, Neuroimaging and the question of neurodegeneration in schizophrenia: Progress in neurobiology, v. 95, no. 4, p. 514-516, doi: 10.1016/j.pneurobio.2011.07.007. 
Mezler, M., Geneste, H., Gault, L., and Marek, G.J., 2010, LY-2140023, a prodrug of the group II metabotropic glutamate receptor agonist LY-404039 for the potential treatment of schizophrenia: Current opinion in investigational drugs (London, England: 2000), v. 11, no. 7, p. 833-845.

Michailov, G.V., Sereda, M.W., Brinkmann, B.G., Fischer, T.M., Haug, B., Birchmeier, C., Role, L., Lai, C., Schwab, M.H., and Nave, K.-A., 2004, Axonal neuregulin-1 regulates myelin sheath thickness: Science (New York, N.Y.), v. 304, no. 5671, p. 700-703, doi: 10.1126/science.1095862.

Millar, J.K., Wilson-Annan, J.C., Anderson, S., Christie, S., Taylor, M.S., Semple, C.A., Devon, R.S., St Clair, D.M., Muir, W.J., Blackwood, D.H., and Porteous, D.J., 2000, Disruption of two novel genes by a translocation co-segregating with schizophrenia: Human molecular genetics, v. 9, no. 9, p. 1415-1423.

Missios, S., 2007, Hippocrates, Galen, and the uses of trepanation in the ancient classical world: Neurosurgical focus, v. 23, no. 1, p. E11, doi: 10.3171/foc.2007.23.1.11.

Mohit, A., 2001, Mental health and psychiatry in the Middle East: historical development: Eastern Mediterranean health journal $=$ La revue de santé de la Méditerranée orientale = al-Majallah al-șiḥhīyah li-sharq al-mutawassiț, v. 7, no. 3, p. 336-347.

Monji, A., Kato, T., and Kanba, S., 2009, Cytokines and schizophrenia: Microglia hypothesis of schizophrenia: Psychiatry and clinical neurosciences, v. 63, no. 3, p. 257-265.

Morton, J.F., 1968, Medicinal plants--old and new: Bulletin of the Medical Library Association, v. 56 , no. 2 , p. $161-167$.

Mülhardt, C., 2003, Der Experimentator, Molekularbiologie/Genomics, Spektrum

Mullis, K.B., and Faloona, F.A., 1987, Specific synthesis of DNA in vitro via a polymerasecatalyzed chain reaction: Methods in enzymology, v. 155, p. 335-350.

Mullis, K., Faloona, F., Scharf, S., Saiki, R., Horn, G., and Erlich, H., 1992, Specific enzymatic amplification of DNA in vitro: the polymerase chain reaction. 1986: Biotechnology (Reading, Mass.), v. 24, p. 17-27.

Munafò, M.R., Thiselton, D.L., Clark, T.G., and Flint, J., 2006, Association of the NRG1 gene and schizophrenia: a meta-analysis: Molecular psychiatry, v. 11, no. 6, p. 539-546, doi: $10.1038 /$ sj.mp.4001817.

Nayak, A., Gayen, P., Saini, P., Maitra, S., and Sinha Babu, S.P., 2011, Albendazole induces apoptosis in adults and microfilariae of Setaria cervi: Experimental parasitology, v. 128 , no. 3, p. 236-242, doi: 10.1016/j.exppara.2011.03.005.

Neddens, J., and Buonanno, A., 2011, Expression of the neuregulin receptor ErbB4 in the brain of the rhesus monkey (Macaca mulatta): PloS one, v. 6, no. 11, p. e27337, doi: 10.1371/journal.pone.0027337.

Neddens, J., and Buonanno, A., 2010, Selective populations of hippocampal interneurons express ErbB4 and their number and distribution is altered in ErbB4 knockout mice: Hippocampus, v. 20, no. 6, p. 724-744, doi: 10.1002/hipo.20675. 
Neddens, J., Fish, K.N., Tricoire, L., Vullhorst, D., Shamir, A., Chung, W., Lewis, D.A., McBain, C.J., and Buonanno, A., 2011, Conserved interneuron-specific ErbB4 expression in frontal cortex of rodents, monkeys, and humans: implications for schizophrenia: Biological psychiatry, v. 70 , no. 7 , p. 636-645, doi: 10.1016/j.biopsych.2011.04.016.

Nestler, E.J., and Hyman, S.E., 2010, Animal models of neuropsychiatric disorders: Nature neuroscience, v. 13 , no. 10, p. 1161-1169, doi: 10.1038/nn.2647.

Ni, C.Y., Murphy, M.P., Golde, T.E., and Carpenter, G., 2001, gamma -Secretase cleavage and nuclear localization of ErbB-4 receptor tyrosine kinase: Science (New York, N.Y.), v. 294 , no. 5549 , p. 2179-2181, doi: 10.1126/science.1065412.

Nicodemus, K.K., Marenco, S., Batten, A.J., Vakkalanka, R., Egan, M.F., Straub, R.E., and Weinberger, D.R., 2008, Serious obstetric complications interact with hypoxiaregulated/vascular-expression genes to influence schizophrenia risk: Molecular psychiatry, v. 13, no. 9, p. 873-877, doi: 10.1038/sj.mp.4002153.

Nilsson, M., Carlsson, A., Markinhuhta, K.R., Sonesson, C., Pettersson, F., Gullme, M., and Carlsson, M.L., 2004, The dopaminergic stabiliser ACR16 counteracts the behavioural primitivization induced by the NMDA receptor antagonist MK-801 in mice: implications for cognition: Progress in neuro-psychopharmacology \& biological psychiatry, v. 28, no. 4, p. 677-685, doi: 10.1016/j.pnpbp.2004.05.004.

Nitsche, M.A., Müller-Dahlhaus, F., Paulus, W., and Ziemann, U., 2012, The pharmacology of neuroplasticity induced by non-invasive brain stimulation: building models for the clinical use of CNS active drugs: The Journal of physiology,, doi: 10.1113/jphysiol.2012.232975.

O'Leary, J., and Muggia, F.M., 1998a, Camptothecins: a review of their development and schedules of administration: European journal of cancer (Oxford, England: 1990), v. 34 , no. 10 , p. $1500-1508$.

Oda, K., Matsuoka, Y., Funahashi, A., and Kitano, H., 2005, A comprehensive pathway map of epidermal growth factor receptor signaling: Molecular Systems Biology, v. 1, p. 2005.0010, doi: 10.1038/msb4100014.

Ogden, D.A., Scherr, L., Spritz, N., and Rubin, A.L., 1961, A comparison of the properties of chlorothiazide, spironolactone and a combination of both as diuretic agents: The New England journal of medicine, v. 265, p. 358-362, doi: 10.1056/NEJM196108242650802.

Ohlmeyer, M., and Zhou, M.-M., 2010, Integration of small-molecule discovery in academic biomedical research: The Mount Sinai journal of medicine, New York, v. 77, no. 4, p. 350-357, doi: 10.1002/msj.20197.

Okasha, A., 2001, Egyptian contribution to the concept of mental health: Eastern Mediterranean health journal $=$ La revue de santé de la Méditerranée orientale $=$ alMajallah al-șị̣hīyah li-sharq al-mutawassiț, v. 7, no. 3, p. 377-380.

Okasha, A., 1999, Mental health in the Middle East: an Egyptian perspective: Clinical psychology review, v. 19, no. 8, p. 917-933. 
Olayioye, M.A., Beuvink, I., Horsch, K., Daly, J.M., and Hynes, N.E., 1999, ErbB receptorinduced activation of stat transcription factors is mediated by Src tyrosine kinases: The Journal of biological chemistry, v. 274, no. 24, p. 17209-17218.

Olney, J.W., and Farber, N.B., 1995, NMDA antagonists as neurotherapeutic drugs, psychotogens, neurotoxins, and research tools for studying schizophrenia: Neuropsychopharmacology: official publication of the American College of Neuropsychopharmacology, v. 13 , no. 4, p. 335-345, doi: 10.1016/0893133X(95)00079-S.

Oyamada, N., Sone, M., Miyashita, K., Park, K., Taura, D., Inuzuka, M., Sonoyama, T., Tsujimoto, H., Fukunaga, Y., Tamura, N., Itoh, H., and Nakao, K., 2008, The role of mineralocorticoid receptor expression in brain remodeling after cerebral ischemia: Endocrinology, v. 149, no. 8, p. 3764-3777, doi: 10.1210/en.2007-1770.

Ozaki, M., Sasner, M., Yano, R., Lu, H.S., and Buonanno, A., 1997, Neuregulin-beta induces expression of an NMDA-receptor subunit: Nature, v. 390, no. 6661, p. 691-694, doi: $10.1038 / 37795$.

Pelicci, G., Lanfrancone, L., Grignani, F., McGlade, J., Cavallo, F., Forni, G., Nicoletti, I., Grignani, F., Pawson, T., and Pelicci, P.G., 1992, A novel transforming protein (SHC) with an SH2 domain is implicated in mitogenic signal transduction: Cell, v. 70, no. 1, p. 93-104.

Penzes, P., Cahill, M.E., Jones, K.A., VanLeeuwen, J.-E., and Woolfrey, K.M., 2011, Dendritic spine pathology in neuropsychiatric disorders: Nature neuroscience, v. 14, no. 3, p. 285-293, doi: $10.1038 / n n .2741$.

Pippal, J.B., and Fuller, P.J., 2008, Structure-function relationships in the mineralocorticoid receptor: Journal of molecular endocrinology, v. 41, no. 6, p. 405-413, doi: 10.1677/JME-08-0093.

Plowman, G.D., Culouscou, J.M., Whitney, G.S., Green, J.M., Carlton, G.W., Foy, L., Neubauer, M.G., and Shoyab, M., 1993, Ligand-specific activation of HER4/p180erbB4, a fourth member of the epidermal growth factor receptor family: Proceedings of the National Academy of Sciences of the United States of America, v. 90 , no. 5 , p. $1746-1750$.

Pommier, Y., 2004, Camptothecins and topoisomerase I: a foot in the door. Targeting the genome beyond topoisomerase I with camptothecins and novel anticancer drugs: importance of DNA replication, repair and cell cycle checkpoints: Current medicinal chemistry. Anti-cancer agents, v. 4, no. 5, p. 429-434.

Pommier, Y., 2006, Topoisomerase I inhibitors: camptothecins and beyond: Nature reviews. Cancer, v. 6, no. 10, p. 789-802, doi: 10.1038/nrc1977.

Pratt, J., Winchester, C., Dawson, N., and Morris, B., 2012, Advancing schizophrenia drug discovery: optimizing rodent models to bridge the translational gap: Nature reviews. Drug discovery, v. 11 , no. 7 , p. 560-579, doi: $10.1038 /$ nrd3649.

Ravichandran, K.S., 2001, Signaling via Shc family adapter proteins: Oncogene, v. 20, no. 44, p. $6322-6330$, doi: $10.1038 /$ sj.onc. 1204776 . 
Redinbo, M.R., Stewart, L., Kuhn, P., Champoux, J.J., and Hol, W.G., 1998, Crystal structures of human topoisomerase I in covalent and noncovalent complexes with DNA: Science (New York, N.Y.), v. 279, no. 5356, p. 1504-1513.

Rio, C., Buxbaum, J.D., Peschon, J.J., and Corfas, G., 2000, Tumor necrosis factor-alphaconverting enzyme is required for cleavage of erbB4/HER4: The Journal of biological chemistry, v. 275 , no. 14 , p. 10379-10387.

Rogerson, F.M., Yao, Y.-Z., Smith, B.J., Dimopoulos, N., and Fuller, P.J., 2003, Determinants of spironolactone binding specificity in the mineralocorticoid receptor: Journal of molecular endocrinology, v. 31, no. 3, p. 573-582.

van Rossum, J.M., 1967, [The effect of psychostimulants on the central and autonomic nervous system]: Schweizerische Zeitschrift für Sportmedizin, v. 15, no. 1, p. 26-40.

van Rossum, J.M., 1966, The significance of dopamine-receptor blockade for the mechanism of action of neuroleptic drugs: Archives internationales de pharmacodynamie et de thérapie, v. 160, no. 2, p. 492-494.

Rowinsky, E.K., Adjei, A., Donehower, R.C., Gore, S.D., Jones, R.J., Burke, P.J., Cheng, Y.C., Grochow, L.B., and Kaufmann, S.H., 1994, Phase I and pharmacodynamic study of the topoisomerase I-inhibitor topotecan in patients with refractory acute leukemia: Journal of clinical oncology: official journal of the American Society of Clinical Oncology, v. 12, no. 10, p. 2193-2203.

Rund, B.R., 2009, Is there a degenerative process going on in the brain of people with Schizophrenia?: Frontiers in human neuroscience, v. 3, p. 36 , doi: 10.3389/neuro.09.036.2009.

Russel, J., Cohn, R., History of schizophrenia, Bookvika publishing

Rumsey, D., 2010, Statistik für Dummies, Wiley

Saha, S., Chant, D., Welham, J., and McGrath, J., 2005, A systematic review of the prevalence of schizophrenia: PLoS medicine, v. 2, no. 5, p. e141, doi: 10.1371/journal.pmed.0020141.

Sams-Dodd, F., Lipska, B.K., and Weinberger, D.R., 1997, Neonatal lesions of the rat ventral hippocampus result in hyperlocomotion and deficits in social behaviour in adulthood: Psychopharmacology, v. 132, no. 3, p. 303-310.

Sanyal, S., and Van Tol, H.H., 1997, Review the role of dopamine D4 receptors in schizophrenia and antipsychotic action: Journal of psychiatric research, v. 31, no. 2, p. 219-232.

Sato, K., Kimoto, M., Kakumoto, M., Horiuchi, D., Iwasaki, T., Tokmakov, A.A., and Fukami, Y., 2000, Adaptor protein Shc undergoes translocation and mediates up-regulation of the tyrosine kinase c-Src in EGF-stimulated A431 cells: Genes to cells: devoted to molecular \& cellular mechanisms, v. 5, no. 9, p. 749-764.

van Schalkwyk, P.C., Geyser, T.L., Récio, M., and Erasmus, F.P., 1979, The anthelmintic efficacy of albendazole against gastrointestinal roundworms, tapeworms, lungworms 
and liverflukes in sheep: Journal of the South African Veterinary Association, v. 50, no. 1 , p. 31-35.

Schulze, W.X., Deng, L., and Mann, M., 2005, Phosphotyrosine interactome of the ErbBreceptor kinase family: Molecular systems biology, v. 1, p. 2005.0008, doi: $10.1038 / \mathrm{msb} 4100012$.

Sebat, J., Levy, D.L., and McCarthy, S.E., 2009, Rare structural variants in schizophrenia: one disorder, multiple mutations; one mutation, multiple disorders: Trends in genetics: TIG, v. 25, no. 12, p. 528-535, doi: 10.1016/j.tig.2009.10.004.

Seeman, P., and Lee, T., 1975, Antipsychotic drugs: direct correlation between clinical potency and presynaptic action on dopamine neurons: Science (New York, N.Y.), v. 188 , no. 4194 , p. 1217-1219.

Silberberg, G., Darvasi, A., Pinkas-Kramarski, R., and Navon, R., 2006, The involvement of ErbB4 with schizophrenia: association and expression studies: American journal of medical genetics. Part B, Neuropsychiatric genetics: the official publication of the International Society of Psychiatric Genetics, v. 141B, no. 2, p. 142-148, doi: 10.1002/ajmg.b.30275.

Sink, R., Gobec, S., Pečar, S., and Zega, A., 2010, False positives in the early stages of drug discovery: Current medicinal chemistry, v. 17, no. 34, p. 4231-4255.

Slichenmyer, W.J., Elliott, W.L., and Fry, D.W., 2001, Cl-1033, a pan-erbB tyrosine kinase inhibitor: Seminars in oncology, v. 28, no. 5 Suppl 16, p. 80-85.

Snyder, S.H., 1980, Phencyclidine: Nature, v. 285, no. 5764, p. 355-356.

St Clair, D., Blackwood, D., Muir, W., Carothers, A., Walker, M., Spowart, G., Gosden, C., and Evans, H.J., 1990, Association within a family of a balanced autosomal translocation with major mental illness: Lancet, v. 336, no. 8706, p. 13-16.

Stagljar, I., Korostensky, C., Johnsson, N., and te Heesen, S., 1998, A genetic system based on split-ubiquitin for the analysis of interactions between membrane proteins in vivo: Proceedings of the National Academy of Sciences of the United States of America, v. 95, no. 9, p. 5187-5192.

Stallings-Mann, M., Jamieson, L., Regala, R.P., Weems, C., Murray, N.R., and Fields, A.P., 2006, A novel small-molecule inhibitor of protein kinase Ciota blocks transformed growth of non-small-cell lung cancer cells: Cancer research, v. 66, no. 3, p. 17671774, doi: 10.1158/0008-5472.CAN-05-3405.

Stefansson, H., Sarginson, J., Kong, A., Yates, P., Steinthorsdottir, V., Gudfinnsson, E., Gunnarsdottir, S., Walker, N., Petursson, H., Crombie, C., Ingason, A., Gulcher, J.R., Stefansson, K., and St Clair, D., 2003, Association of neuregulin 1 with schizophrenia confirmed in a Scottish population: American journal of human genetics, v. 72, no. 1, p. 83-87.

Stefansson, H., Sigurdsson, E., Steinthorsdottir, V., Bjornsdottir, S., Sigmundsson, T., Ghosh, S., Brynjolfsson, J., Gunnarsdottir, S., Ivarsson, O., Chou, T.T., Hjaltason, O., Birgisdottir, B., Jonsson, H., Gudnadottir, V.G., et al., 2002, Neuregulin 1 and 
susceptibility to schizophrenia: American journal of human genetics, v. 71 , no. 4 , p. 877-892, doi: 10.1086/342734.

Stefánsson, H., Thorgeirsson, T.E., Gulcher, J.R., and Stefánsson, K., 2003, Neuregulin 1 in schizophrenia: out of Iceland: Molecular psychiatry, v. 8, no. 7, p. 639-640, doi: 10.1038/sj.mp.4001384.

Steurer, J., Bachmann, L.M., and Miettinen, O.S., 2006, Etiology in a taxonomy of illnesses: European journal of epidemiology, v. 21, no. 2, p. 85-89, doi: 10.1007/s10654-0055925-4.

Streltsov, S., Oleinikov, V., Ermishov, M., Mochalov, K., Sukhanova, A., Nechipurenko, Y., Grokhovsky, S., Zhuze, A., Pluot, M., and Nabiev, I., 2003, Interaction of clinically important human DNA topoisomerase I poison, topotecan, with double-stranded DNA: Biopolymers, v. 72, no. 6, p. 442-454, doi: 10.1002/bip.10479.

Stryer,L., Berg,M., Tymoczko, L., 2007 Biochemie, Spektrum Akademischer Verlag

Sui, Y., and Wu, Z., 2007, Alternative statistical parameter for high-throughput screening assay quality assessment: Journal of biomolecular screening, v. 12, no. 2, p. 229234, doi: $10.1177 / 1087057106296498$.

Sullivan, P.F., Kendler, K.S., and Neale, M.C., 2003, Schizophrenia as a complex trait: evidence from a meta-analysis of twin studies: Archives of general psychiatry, v. 60, no. 12 , p. 1187-1192, doi: 10.1001/archpsyc.60.12.1187.

Sun, H., Liu, X., Xiong, Q., Shikano, S., and Li, M., 2006, Chronic inhibition of cardiac Kir2.1 and HERG potassium channels by celastrol with dual effects on both ion conductivity and protein trafficking: The Journal of biological chemistry, v. 281 , no. 9, p. 58775884, doi: 10.1074/jbc.M600072200.

Taguchi, T., Wakui, A., Hasegawa, K., Niitani, H., Furue, H., Ohta, K., and Hattori, T., 1990, [Phase I clinical study of CPT-11. Research group of CPT-11]: Gan to kagaku ryoho. Cancer \& chemotherapy, v. 17 , no. 1, p. 115-120.

Takimoto, C.H., and Arbuck, S.G., 1997a, Clinical status and optimal use of topotecan: Oncology (Williston Park, N.Y.), v. 11, no. 11, p. 1635-1646; discussion 1649-1651, 1655-1657.

Takimoto, C.H., and Arbuck, S.G., 1997b, Clinical status and optimal use of topotecan: Oncology (Williston Park, N.Y.), v. 11, no. 11, p. 1635-1646; discussion 1649-1651, 1655-1657.

Tandon, R., Keshavan, M.S., and Nasrallah, H.A., 2008a, Schizophrenia, "just the facts" what we know in 2008. 2. Epidemiology and etiology: Schizophrenia research, v. 102, no. 1-3, p. 1-18, doi: 10.1016/j.schres.2008.04.011.

Tandon, R., Keshavan, M.S., and Nasrallah, H.A., 2008b, Schizophrenia, "Just the Facts": What we know in 2008: Part 1: Overview: Schizophrenia Research, v. 100, no. 1-3, p. 4-19, doi: 10.1016/j.schres.2008.01.022. 
Tandon, R., Nasrallah, H.A., and Keshavan, M.S., 2009, Schizophrenia, "just the facts" 4. Clinical features and conceptualization: Schizophrenia research, v. 110, no. 1-3, p. 123, doi: 10.1016/j.schres.2009.03.005.

Tandon, R., Nasrallah, H.A., and Keshavan, M.S., 2010, Schizophrenia, "just the facts" 5. Treatment and prevention. Past, present, and future: Schizophrenia research, v. 122, no. 1-3, p. 1-23, doi: 10.1016/j.schres.2010.05.025.

Taniguchi, C.M., Emanuelli, B., and Kahn, C.R., 2006, Critical nodes in signalling pathways: insights into insulin action: Nature reviews. Molecular cell biology, v. 7, no. 2, p. 8596, doi: 10.1038/nrm1837.

Taveggia, C., Thaker, P., Petrylak, A., Caporaso, G.L., Toews, A., Falls, D.L., Einheber, S., and Salzer, J.L., 2008, Type III neuregulin-1 promotes oligodendrocyte myelination: Glia, v. 56, no. 3, p. 284-293, doi: 10.1002/glia.20612.

Taveggia, C., Zanazzi, G., Petrylak, A., Yano, H., Rosenbluth, J., Einheber, S., Xu, X., Esper, R.M., Loeb, J.A., Shrager, P., Chao, M.V., Falls, D.L., Role, L., and Salzer, J.L., 2005, Neuregulin-1 type III determines the ensheathment fate of axons: Neuron, v. 47, no. 5, p. 681-694, doi: 10.1016/j.neuron.2005.08.017.

Tidcombe, H., Jackson-Fisher, A., Mathers, K., Stern, D.F., Gassmann, M., and Golding, J.P., 2003, Neural and mammary gland defects in ErbB4 knockout mice genetically rescued from embryonic lethality: Proceedings of the National Academy of Sciences of the United States of America, v. 100, no. 14, p. 8281-8286, doi: 10.1073/pnas. 1436402100 .

Ting, A.K., Chen, Y., Wen, L., Yin, D.-M., Shen, C., Tao, Y., Liu, X., Xiong, W.-C., and Mei, L., 2011, Neuregulin 1 promotes excitatory synapse development and function in GABAergic interneurons: The Journal of neuroscience: the official journal of the Society for Neuroscience, v. 31, no. 1, p. 15-25, doi: 10.1523/JNEUROSCI.253810.2011.

Towbin, H., Staehelin, T., and Gordon, J., 1992, Electrophoretic transfer of proteins from polyacrylamide gels to nitrocellulose sheets: procedure and some applications. 1979: Biotechnology (Reading, Mass.), v. 24, p. 145-149.

Tsai, G., and Coyle, J.T., 2002, Glutamatergic mechanisms in schizophrenia: Annual review of pharmacology and toxicology, v. 42, p. 165-179, doi: 10.1146/annurev.pharmtox.42.082701.160735.

Tzahar, E., Waterman, H., Chen, X., Levkowitz, G., Karunagaran, D., Lavi, S., Ratzkin, B.J., and Yarden, Y., 1996, A hierarchical network of interreceptor interactions determines signal transduction by Neu differentiation factor/neuregulin and epidermal growth factor: Molecular and cellular biology, v. 16, no. 10, p. 5276-5287.

Uetz, P., and Hughes, R.E., 2000, Systematic and large-scale two-hybrid screens: Current opinion in microbiology, v. 3, no. 3, p. 303-308.

Uhlhaas, P.J., and Singer, W., 2010, Abnormal neural oscillations and synchrony in schizophrenia: Nature reviews. Neuroscience, v. 11, no. 2, p. 100-113, doi: 10.1038/nrn2774. 
Velanac, V., Unterbarnscheidt, T., Hinrichs, W., Gummert, M.N., Fischer, T.M., Rossner, M.J., Trimarco, A., Brivio, V., Taveggia, C., Willem, M., Haass, C., Möbius, W., Nave, K.-A., and Schwab, M.H., 2012, Bace1 processing of NRG1 type III produces a myelininducing signal but is not essential for the stimulation of myelination: Glia, v. 60, no. 2, p. 203-217, doi: 10.1002/glia.21255.

Vidal, G.A., Naresh, A., Marrero, L., and Jones, F.E., 2005, Presenilin-dependent gammasecretase processing regulates multiple ERBB4/HER4 activities: The Journal of biological chemistry, v. 280, no. 20, p. 19777-19783, doi: 10.1074/jbc.M412457200.

Vigano, D., Guidali, C., Petrosino, S., Realini, N., Rubino, T., Di Marzo, V., and Parolaro, D., 2009, Involvement of the endocannabinoid system in phencyclidine-induced cognitive deficits modelling schizophrenia: The international journal of neuropsychopharmacology / official scientific journal of the Collegium Internationale Neuropsychopharmacologicum (CINP), v. 12, no. 5, p. 599-614, doi: 10.1017/S1461145708009371.

Wehr, M.C., Laage, R., Bolz, U., Fischer, T.M., Grünewald, S., Scheek, S., Bach, A., Nave, K.-A., and Rossner, M.J., 2006, Monitoring regulated protein-protein interactions using split TEV: Nature methods, v. 3, no. 12, p. 985-993, doi: 10.1038/nmeth967.

Wehr, M.C., Reinecke, L., Botvinnik, A., and Rossner, M.J., 2008a, Analysis of transient phosphorylation-dependent protein-protein interactions in living mammalian cells using split-TEV: BMC biotechnology, v. 8, p. 55, doi: 10.1186/1472-6750-8-55.

Weickert, C.S., Tiwari, Y., Schofield, P.R., Mowry, B.J., and Fullerton, J.M., 2012, Schizophrenia-associated HapICE haplotype is associated with increased NRG1 type III expression and high nucleotide diversity: Translational Psychiatry, v. 2, no. 4, p. e104, doi: 10.1038/tp.2012.25.

Weisman, J.L., Liou, A.P., Shelat, A.A., Cohen, F.E., Guy, R.K., and DeRisi, J.L., 2006, Searching for new antimalarial therapeutics amongst known drugs: Chemical biology \& drug design, v. 67, no. 6, p. 409-416, doi: 10.1111/j.1747-0285.2006.00391.x.

Weissmann, C., and Aguzzi, A., 2005, Approaches to therapy of prion diseases: Annual review of medicine, v. 56, p. 321-344, doi: 10.1146/annurev.med.56.062404.172936.

Wen, L., Lu, Y.-S., Zhu, X.-H., Li, X.-M., Woo, R.-S., Chen, Y.-J., Yin, D.-M., Lai, C., Terry, A.V., Jr, Vazdarjanova, A., Xiong, W.-C., and Mei, L., 2010, Neuregulin 1 regulates pyramidal neuron activity via ErbB4 in parvalbumin-positive interneurons: Proceedings of the National Academy of Sciences of the United States of America, $v$. 107, no. 3, p. 1211-1216, doi: 10.1073/pnas.0910302107.

Wexler, E.M., and Geschwind, D.H., 2011, DISC1: a schizophrenia gene with multiple personalities: Neuron, v. 72, no. 4, p. 501-503, doi: 10.1016/j.neuron.2011.10.023.

Whalley, H.C., Papmeyer, M., Sprooten, E., Lawrie, S.M., Sussmann, J.E., and McIntosh, A.M., 2012, Review of functional magnetic resonance imaging studies comparing bipolar disorder and schizophrenia: Bipolar disorders, v. 14, no. 4, p. 411-431, doi: 10.1111/j.1399-5618.2012.01016.x.

Williams, C.C., Allison, J.G., Vidal, G.A., Burow, M.E., Beckman, B.S., Marrero, L., and Jones, F.E., 2004, The ERBB4/HER4 receptor tyrosine kinase regulates gene expression by 
functioning as a STAT5A nuclear chaperone: The Journal of cell biology, v. 167, no. 3, p. 469-478, doi: 10.1083/jcb.200403155.

Williams, R., Berndt, A., Miller, S., Hon, W.-C., and Zhang, X., 2009, Form and flexibility in phosphoinositide 3-kinases: Biochemical Society transactions, v. 37, no. Pt 4, p. 615626, doi: 10.1042/BST0370615.

Williamson, P, 2005, Mind Brain and Schiziophrenia, Oxford University Press

Winterer, G., Konrad, A., Vucurevic, G., Musso, F., Stoeter, P., and Dahmen, N., 2008, Association of $5^{\prime}$ end neuregulin-1 (NRG1) gene variation with subcortical medial frontal microstructure in humans: Neurolmage, v. 40 , no. 2, p. $712-718$, doi: 10.1016/j.neuroimage.2007.12.041.

Woo, T.U., Whitehead, R.E., Melchitzky, D.S., and Lewis, D.A., 1998, A subclass of prefrontal gamma-aminobutyric acid axon terminals are selectively altered in schizophrenia: Proceedings of the National Academy of Sciences of the United States of America, v. 95 , no. 9 , p. 5341-5346.

Xie, M., Yang, D., Wu, M., Xue, B., and Yan, B., 2003, Mouse liver and kidney carboxylesterase (M-LK) rapidly hydrolyzes antitumor prodrug irinotecan and the Nterminal three quarter sequence determines substrate selectivity: Drug metabolism and disposition: the biological fate of chemicals, v. 31, no. 1, p. 21-27.

Yap, T.A., Garrett, M.D., Walton, M.I., Raynaud, F., de Bono, J.S., and Workman, P., 2008, Targeting the PI3K-AKT-mTOR pathway: progress, pitfalls, and promises: Current opinion in pharmacology, v. 8, no. 4, p. 393-412, doi: 10.1016/j.coph.2008.08.004.

Yarden, Y., and Pines, G., 2012, The ERBB network: at last, cancer therapy meets systems biology: Nature Reviews. Cancer, v. 12, no. 8, p. 553-563, doi: 10.1038/nrc3309.

Yarden, Y., and Sliwkowski, M.X., 2001, Untangling the ErbB signalling network: Nature reviews. Molecular cell biology, v. 2, no. 2, p. 127-137, doi: 10.1038/35052073.

Zamboni, W.C., Houghton, P.J., Thompson, J., Cheshire, P.J., Hanna, S.K., Richmond, L.B., Lou, X., and Stewart, C.F., 1998, Altered irinotecan and SN-38 disposition after intravenous and oral administration of irinotecan in mice bearing human neuroblastoma xenografts: Clinical cancer research: an official journal of the American Association for Cancer Research, v. 4, no. 2, p. 455-462.

Zeng, F., Zhang, M.-Z., Singh, A.B., Zent, R., and Harris, R.C., 2007a, ErbB4 isoforms selectively regulate growth factor induced Madin-Darby canine kidney cell tubulogenesis: Molecular biology of the cell, v. 18, no. 11, p. 4446-4456, doi: 10.1091/mbc.E07-03-0223.

Zhang, X.D., 2008, Novel analytic criteria and effective plate designs for quality control in genome-scale RNAi screens: Journal of biomolecular screening, v. 13, no. 5, p. 363377, doi: $10.1177 / 1087057108317062$.

Zhang, Chung, and Oldenburg, 1999, A Simple Statistical Parameter for Use in Evaluation and Validation of High Throughput Screening Assays: Journal of biomolecular screening, v. 4, no. 2, p. 67-73. 
Zhang, X.D., Espeseth, A.S., Johnson, E.N., Chin, J., Gates, A., Mitnaul, L.J., Marine, S.D., Tian, J., Stec, E.M., Kunapuli, P., Holder, D.J., Heyse, J.F., Strulovici, B., and Ferrer, M., 2008, Integrating experimental and analytic approaches to improve data quality in genome-wide RNAi screens: Journal of biomolecular screening, v. 13, no. 5, p. 378389, doi: $10.1177 / 1087057108317145$. 